DCRE --53713

DE86 007792

\title{
Physical Processes in Collapse Driven Supernova
}

\author{
R. W. Mayle \\ (Ph.D. Thesis)
}

Manuscript date: November 25, 1985

\section{DISCLAIMER}

\begin{abstract}
This report was prepared as as stciust of work sponsored by at apency of the United Sebtes Governmeat. Neither the Unied Stutes Government noe any acency therwor, nor any of their

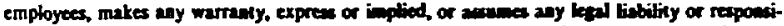
bility for the accurecy, completenes, of enefuleses of any infornation, apperatus, prodest, or process disclowed, or represents that its we would wo iafriese privalety onted riphts Refer-

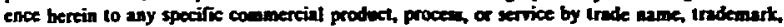

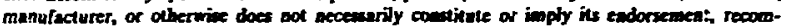
meadation, of fovocing by the United States Covernmeat or any ayeacy thereof. The views

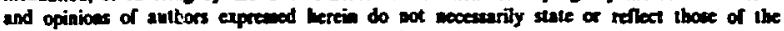
Uaited States Cowerameat or any ascacy thereof.
\end{abstract}

\section{LAWRENCE LIVERMORE NATIONAL LABORATORY University of California - Livermore, California - 94550}


Physical Processes in Collapse Driven Supernova

By

Ronald Willis Male

B.S. (University of Nebraska, Lincoln) 1976

M.S. (University of Nebraska, Lincoln) 1979

DISSERTATION

Submitted in partial satisfaction of the requirements for the degree of DOCTOR OF PHILOSOPHy

in

Physics

. in the

GRADUATE DIVISION

OF TIE

UNIVERSITY OF CALIFORNIA, BERNELEY

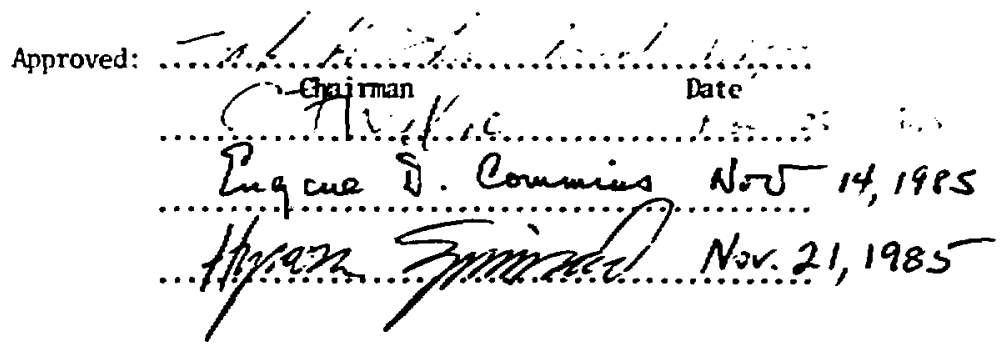




\title{
PHYSICAL PROCESSES IN COLLAPSE DRIVEN SUPERNOVA
}

\author{
Ronald W. Mayle
}

\begin{abstract}
Supernova ere believed to occur when a mavive star, unable to continue burning nuclear fuel in its interior, collapes, and aubequently produces epectactular explosion. The understanding of how this occurs ha been the mubject of much research over the years, jel the exact acenario hes been elusive. James R. Wilson, in 1982, discovered what may be the solution to this problem. The explosive mecharism he found relies on the fact that the central part of these massive atars evolve into neutron atars, and in so doing, emit about $10^{53}$ ergs of energy in neutrinos. About one second after the core of the star has collapscd to nuclear densities, thermodynamic conditions in the region behind the accretion shock (produced as the core halted and bcunced) and outside the neutrinosphere, are favorable for free baryons to absorb the hot core neutrinos, and gain thermal energy- This gain of energy produces material pressure that pushes on the accretion shock, and helps it to motc i.2to the stellar envelope. Work that continues the investigation of these late time supc:nova (so called because it was previously thought that, after bounce, an explosion would occur on timescales of tens of milliseconds) is reported in this thesis. The method of neutrino transport being used in a numerical simulation is very important in the sludy of late time supernova, as the explosive mechanism depends on neutrino heating of the material behind the shock. Neutrino transport is discussed, and results are presented that indicate flux limited neutrino iransport does an adequate job of following the Deutrino evolution. The core region of these exploding stars becomes unstable to convective motions during the supernova evolution. Convective mixing allows more neutrinos to escape from under the neutrinosphere, and thus increases the amount of heating by neutrinos. An approximate method of incorporating convection is described, and some results of including convection in a computer model is presented. Another phenomena is seen in computer simulations of oupernova, cscillations in the neutrino luminosity and mass accretion rate onto the protoneutron star. The last topic discussed in this thesis describes the attempt to understand this oscillation by perturbation of the steady state solution to equations approximating the comp ex physical processes occuring in the late time supernova.
\end{abstract}




\section{CONTENTS}

\section{CHAPTERS}

I. A Short Hintory of Supernove 1

I. Qualitative Deacription of a Collapwe Driven Supernova 5

III. Equations to Model a Supernova 30

IV. Neutrino Tranuport 45

V. Convection in Supernove 120

V. Radiative Accretion Shocke 139

VII. Supernove Code Results 176

Appendix A 188

Appendix B 217 
This work is dedirated to my

good friend and teacher

Jamee R. Witson 


\section{Acknowledgement}

There are many factors involved in the successful completion of a PhD degree. Most of which depend on the generosity of people or organizations that help a student turn interest in some subject into a work worthy of recognition. I want to say thank you to the foilowing people who were important to my finishing this project on oupernovae.

Financial support from the Institute for Geophysics and Planetary Physica (IGPP) at Liverınore, California, during the research on supernovec, and support from the state of Californis during the period of time at U.C. Berkeley prior to the beginning of research, allowed me the freedom to pursue a PhD degree.

B division at Lawrence Livermore National Laboratory provided the computer time that is necessary to the numerical study of supernovae.

Frank Shu at Berkeley, gave me the chance to work on aupernovae by introducing me to Jim Wilson at the Lawrence Livermore National Laboratory, and allowirg me to work under Jim's supervision. Jim Wilson gave much of his time helping me to the point where I could do research on the supernova problem, and has been a constant soirce of encouragement.

Others at B division have been important to my success, especially Jim LeBlanc, whose discussions with Jim Wilson and myself, and with myself alone proved to be very helpful Nancy Alexander answered questions from a first time user of a computer system with kind patience. The people in B division have shown themselves to be good hosts.

Stanford Woosley, at the University of California at Santa Cruz, has become a good friend and collaborator in research on supernovae, and has provided the initial models used in our study of supernovae.

At the Lawrence Berkeley Laboratory, the Kenny-Heimholz group has generously allowed me the use of their word processor to preduce this document.

Personal friendships have given me much needed support during the time spent in graduate study. Sometimes it is difficult to put into words how one feels, but words are all I have to express my appreciation of the friendship extended to me by Brian and Julie Dougherty. I hope the friendship will continue long irsto the future.

Finally, April, a cat I knew for 20 years, and who made the trip with me to California in her $16^{\text {th }}$ year, was a good friend, unjudging, and a good mouser. Without her insistent wake up call for breakfast, I might have overslept, and missed many an opportunity for success in graduate study. 


\section{Chapter I}

\section{A SHORT HISTORY OF SUPERNOYA}

In 1934 Bsade and Zwicky suggested that the bright optical displays (10 10 ergs total output) seen in a omall region of a galaxy, over a time scale of 100 's of dayn, resuited from the explosive death of a star. (Later, in wa dixcovered that abuut $10^{61}$ erga of kinetic energy contained in matter motion is also released during the catartrophic event.) Today, it is said that Baade and Zwicky probably made the correct anocintion.

Hoyle (1946) recognized that a highly erolved otar ( $M \geq 10 M_{0}$ ) with an iron core, would be gravitationaliy unstable to collapec. In this 1946 paper he propoed that such a star would become rotationally unstable as the collapec continued, due to angular momentum conservation. Material would be thrown of a the centrifugal forces overcame gravity and this pruduce a supernova explosion.

Burbidge, Burbidge, Fowler and Hoyle (1957) proponed a different mechanism for the explosion of a supernova. They assumed the gravitational instability propoced previously by Hoyle (1946) would trigger the collapue, and argued that the dynamical implosion accompanying crllapse could result in the rapid compression and heating of the lighter elements surrounding the iron core, and thereby initiate a thermonuclear explosion in this region.

Present day workers in the field of supernove theory probably would agree that some supernova are triggerif by a gravitationally unstable iron core, while others explode due to nuclear energy generated by elements lighter than iron. However, the supernova mechanisms mentioned above are not considered likely, even though the exact scenario is still not completely certain today.

Observers have found that the majority of supernova can be classified as belonging to one of two distinet types. Type I supernova have no hydrogen lines in a spectruin taken at maximum brightness. They are seen in all galaxy types and their light curves tend to be homogeneous. Type II supernova posseas hydrogen lines in the opectrum taken at maximum brightness, are not seen in elliptical galaxies and, as a class, bave light curves that are not as humogeneous as the Type I light curves.

From the observations above, a reasonable association of red giant stars (with iron cores, unable to stably burn nuclear fuel further) as progenitors of Type Il supernova, and white dwarf stars (accreting materia] from a massive companion and approaching the limiting mass for degenerate electron pressure to withstand collapse) as progenitors of Type I supernova 
can be made. The fact that red giants contain a hydrogen envelope and are young compared to stars in an elliptical galaxy, while white dwarfs have shed their hydrogen envelope, are end products of stellar evolution, and are found in any galexy, would be consistent with the observations.

As of the present, a common concensus has been arrived at (see Woosley and Weaver (1986) and references contained therein) that the explosive energy produced by an unstablz white dwarf, and which d.ives the explosion itself, is due to the nuclear reactions that convert. the light elements found in the core of a white dwar (i.e. $\mathrm{C}, \mathrm{O}, \mathrm{Ne}$ ) to iron group elements. The exact method of this burning (i.e. detonation or defiagration) is still undecided. Also agreed is that the energy powering a collapse driven supernova is gravitational energy, as the remnant produced after the explosion, had, in eome way, delivered part or its gravitational binding energy to the material blown away. In the collopse driven aupernova, the mechanism for delivery of the binding energy to the stellar envelope is atill not agreed upon.

Colgate and White (1966) published results of a numerical calculation that obtained explosions from a collapse driven aupernova. Neutrinos exiting the core (produced by the capture of electrons on the core baryons) deposited enough energy (during rare interactions with matter) to give the envelope an escape velocity. This burst or neutrinos lasted about .01 second. They did not use radiation transport to follow the neutrinos, but used instead entropy source and sink terms in the matter internal energy equation to model the effect of neutrinos.

Wilson (1971) published results of numerical work which included neutrino transport in a fully consistent general relativistic calculation modeling a collapse driven supernora. He showed that the neutrino burst was not strong enough to eject material, but that the rebound from collapse could, in low mass, stars start a shock wave that would eject the envelope.

Around the late 60's and into the 70's, the understanding of the weak interaction was undergoing change. The Weinberg-Salam model of the electroweak interaction be: ne the standard theory, and showed new possibilities for neutrino interaction with matter. Neutral weak current interactions were first observed in the laboratory in 1973 (see Commins and Bucksbaum (1983), for an introduction to weak interactions), and are predicted by the Weinberg-Salam model. For the supernova problem, coherent scattering of neutrinos through neutral currents (cross-section proportional to the number of scatterers squared) 
would enhance the coupling between the neutrino and the elements (i.e. $\mathrm{Si}, \mathrm{O}, \mathrm{C}$ ) found in the mantle surrounding the iron core (wee Fredman (1974)). It wa boped this increased cross-section would revive the Colgate and White ccenario for mpernove explosions. Many workers (Wileon(1974), Bruenn(1975), Schramm and Arnett(1975)) included the new weak interaction croos-sections, but iound that exploaions (for the finally accepled neutrino-nucleon coupling (see Weinberg (1972)) atill did not result.

Although it was long recognized that a central part of the utar ( $\left.\rho \geqq_{10}{ }^{11} \frac{\mathrm{s}}{\mathrm{ce}}\right)$ forms where neutrinos do not escape repidly due to the emall neutrino mean free path, it was now becoming apparent that one could gain understanding of the supernove evolution by separating the rtellar structure into two parts, the material inside the neutrinoaphere, and the material in the rest of the star (see Bethe et al. (1979)).

Attention was also being paid to the pomibility that the hydrodynamic bounce of the core would drive a shock wave out into the mantle, and so blow of the envelope of the atar, as was seen by Wilson (1971) for low mass stars. The bounce motivated much research into the equation of state of matter near and above nuclear densities (wee Bethe et al. (1979)).

Numerical modelers (Bowers and Wilson(1982 a,b), Cooperstein (1982)), however, were finding the shock wave stalling on its exit from the core, becoming an accretion shuck. This was due to the loss of energy to neutrinos produced by the high temperature post-shocked material, and by the energy needed to dissociate the heavy elements passing through the shock to free baryons or helium. (See Baron, Cooperstein, and Kahana (1985) for recent work on the prompt hydrodynamic bounce supernova and the equation of state.)

The subject of this thesis concerns a new mechanism for the explosion produced in a collapse driven supernova. Wilson (1985) discovered that, at late times (i.e. about one second after bounce) the accretion shock can be revived. Energy is deposited in the region behind the shock by neutrinos leaving the neutrinosphere, thereby strengthening the shock and allowing it to proceed out into the stellar envelope.

Chapter II gives a qualitative description of the evolution of a supernova, in particular a late time supernova. 


\section{Chapter I References}

Basde, W. and Zwicky, F. 1934, Phys. Rev., v45, 138.

Baron, E. Coopersiein, J. and Kahana, S. 1985, Phys. Rev. Lettr., v55, 126.

Bethe, H.A., Brown, G.E., Applegate,J., and Lattimer, J. 1979, Nuel.Phys., A324, 487.

Bruenn, S.W. 1975, Ann. N.Y.Acad.Sci. v262, 80.

Burbidge, E.M., Burbidge, G.R., Fowler, W.A. and Hoyle, F. 1957 Rev. Mod. Phys., v29, 547.

Colgate, S.A. and White, R.H. 1966, Ap.J. v143, 626.

Commins, E. and Bucksbaum, P. 1983 "Weak Interastions of Leptons and Quarks", (Cambridge University Press:Cambridge), Chaptar 1.

Cooperstein, J. 1982, PhD Thesis, SUNY Stony Brook

Freedman, D.Z. 1974, Phys Rev. D, v9., 1389.

Hoyle, F. 1946, Mon. Not. Roy. Astron. Soc., v106, 343.

Schramm, D.N. and Arnett, W.D. 1975, Ap. J., v198, 629.

Weinberg, S. 1972, Phys. Rev. D, v5, 1412.

Wilson, J.R. 1971, Ap.J. v163, 209.

- 1974, Phys. Rev. Lettr., v32, 849.

- 1985, in "Numerical Astrophysics", cd. J.M. Centralla, J.M. LeBlanc, and R.L. Bowers,(Jones and Bartlett:Boston),p.422

Woosley, S.E. and Weaver, T.A. 1986 Ann. Rev. Astron. and Astro., to be published. 


\section{Chapter II}

\section{QUALITATIVE DESCRIPMON OF A COLLAYSE DRIVTN SUPERNOVA}

Figure 1 ahows a Hertzoprung-Rumeli diegram with the wolid line the main equence,

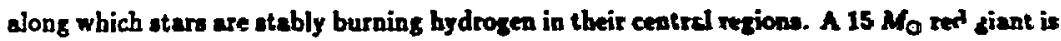
shown in the upper right hand corner of the diagram, and ita prior and nubequeat evolution is described below.

\section{STRUCTURE OF A SUPERNOYA PROGENTOR}

The $15 M_{\odot}$ star remained on the majn neouence while burning hydrogen to belium in the inner $10 \%$ of its mass, where therr.zodynamic conditions were favorable for the production of helium. It remained on the main sequence approximately $10^{7}$ years. This lifetime can re estimated using the fact that 7 Mev per nucleon of nuclear binding energy is liberated when 4 hydrogen nuclei become one He nucleus (prchably mediated by the CXO cycle in this massive star). Using $L \approx 10^{4} L_{\odot}$, fror Ggure 1 :

$$
t_{M S}=\left(\frac{.1 M}{m_{B}}\right)\left(\frac{7 M e v}{L}\right)=1.7 \times 10^{2} \text { yr. } .
$$

where $m_{B}$ is the mass of a proton. After exiausting hydrogen, the central regions contract, raising temperatures and densitiea to the poizt where helium can burn to carbon.

For stars greater than about $8 M_{0}$, thermonuclear burning begins when the electrons are non-degenerate. Since the core is in quasi-equilibrium, the pressure at any radius is determined by the amount of mass above that racius. If the electrons are nondegenerate then $P \propto \rho T$. As the burning begins, if $T$ increases, $\rho$ will deerease (the pressure must star relatively constant). This lowers the reaction rate, and thereby allows the star to adjust .o the new burning conditions without a major disruption. If tive electrons were degenerate, $P \propto \rho^{\gamma}(\gamma \geq 1)$, and when thermonuclear burning beging, there is no stabiliziag feedback to allow the reaction rate to adjust to the atars energy production needs. Thermonuclear burning under degeneraite conditions can result in a major disruption of the star.

During the central core contraction of late stage burning, the stellar radius increases due to the increased luminosity heating and pushing on the outar layers of the star. However, the luminosity increase is only slight, and massive stars ( $M \geqq 10 M_{\odot}$ ) tend to track in the Hertzsprung-Russell diegram in a roughly horizontal manner (see Weaver and Woosley (1980)). 
After exhausting belium, the etar goes through a core contraction phene until carbon bu:ning can begin. In fect, the etar begins to burn elements up to the iron group nuclei, each in succeseion, as it exhausts its current fuel, and core contracts until the next arailable element can be burned. Since benvier elementa relene les binding energ per nucleon, these further stages become much ahorter than the main equence lifetime.

When silicon burning is going on in the core, temperatures are around $5 \mathrm{Mer}$ (see Clayton (1968)). The ion group nuckei produced come into nuclear ataliatical equilibrium as temperatures are now wo high that nuclear reaction rates ars rapid enough to produce equilibration. During this burning stage to iron, neutrino lowes lead to values of temperature and density such that the iron core becomes mildly degeperate before collape. (In the most recent $15 M_{\odot}$ atar erolutionary calculation done by Weaver and Wooley, the inner $1.3 M_{\odot}$ is in NSE at the onvet of collopere.)

Prior to collapse, a hot iron white dwarl size core exists at the center of the atar, surrounded by concentric shells of lighter elemente, remnants of the eurlier burning siages. These shells still are producing energy, but not enough to compensate for the energy lost in radiation from the stellar surface. The main source of presaure suppurt for the iron white dwarf is electron degeneracy pressure.

From energy considerations:

$$
<\varepsilon_{\varepsilon}>\frac{p \bar{Z}}{m_{B}}+\frac{3}{2} \frac{p \dot{\kappa}_{B} T}{56 m_{B}} \approx \frac{\rho G M}{R}
$$

where $\left\langle\epsilon_{t}\right\rangle$ is the average energy per electron, and $\bar{Z}$ is the number of matter elcetrons per baryon. (fany nuclear energy generated during a collapse to radius $\boldsymbol{A}$ is ascumed radiated away.)

Now $<\epsilon_{e}>\approx \frac{3}{4} \mu_{e}$, where $\mu_{e}$ is the electron chemical potential. One finds from (1):

$$
\frac{3}{4} \mu_{\varepsilon} \bar{Z}+\frac{3}{2} \frac{k_{B} T}{56} \approx \frac{G M m_{B}}{R}
$$

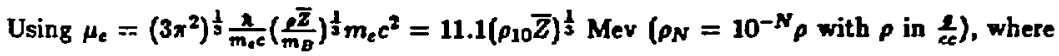
the expression for $\mu_{e}$ is sccurate in the zero temperature limit, and neglecting the second term on the left hand side of (2) results in:

$$
\mu_{e} \approx \frac{4}{3} \frac{G M m_{B}}{R \bar{Z}}
$$

Taking $R \approx 10^{8} \mathrm{~cm}$ (a yical number from evolutionary calculations), $\bar{Z} \approx .46$ for iron, 
and $M \approx M_{C H}$, the limiting men for a white dwarf (i.e. $5.76 Z^{2} M_{0}$ ), the value found for $\mu_{e}$ is about $5.0 \mathrm{Mev}$.

Now the temperature is about .5 Mer (silicon buming temperature) and $00 \frac{\mathrm{ta}}{\mathrm{T}} \sim \frac{1}{10}$. Thus the approximation used in going from equations (2) to (3) it justified. The density can then be determined using $\mu_{10}=\frac{1}{2}\left(\frac{\mu_{0}}{11.1}\right)^{3}$ and $\mu_{c}=5 \mathrm{Mer}$. This sives a value of about $2 \times 10^{\circ} \frac{2}{c c}$ for the central density.

Summarizing this section, a $15 M_{\odot}$ utar evolves to a poins where an iron white dxarf core bas formed. Central temperatures are about $.5 \mathrm{Mer}$ and central dencities are around $2 \times 10^{\circ} \frac{\mathrm{L}}{\mathrm{cc}}$. Iron will not burn to preduce energy, $2 \mathrm{it}$ is the mont tightly bound nucleus. At this time, the character of the evolution of the star changes, due to this inability of iron to burn exothermically.

The next section describes the first event that occurs when the is $M_{0}$ red giant reaches the point where nuclear energy generation in the core is imposible.

\section{COLLAPSE}

A collapse is triggered by the loss of pressure support. Two mechanisms contribute to this loss, iron thermal disintegration and electron capture onto protons. Iron thermal disintegration is discussed first.

Iron Thermal Divintegration

$$
\begin{gathered}
{ }^{6 \mathrm{Fe}} \Longrightarrow{ }^{60} \mathrm{Fe}^{*} \\
{ }^{66} \mathrm{Fe} \Rightarrow 13 \mathrm{He}+4 \mathrm{n}
\end{gathered}
$$

where (4) is a simplification of a large network of equilibrium reactions. ${ }^{56} \mathrm{Fe}^{*}$ indicates an excited state of the jron nucleus. (Note, to change one Fe nucleus into 13 He uclei and 4 neutrons requires about $125 \mathrm{Mev}$.)

Since reactions (4) occur in equilibrium, entropy is not changed by thermal disintegration of iron. However the adiabatic index, $\gamma$, which would be $\frac{1}{3}$ (pressure support by degenerate electrons), drops below this value because of the thermal disintegration. The energy needed to excite or dissociate the iron becomes unavailable for thermal pressure support.

The internal energy of the core is dominated at the onset of collapse by the semidegenerate electron gas. From equation (15) in Chapter $V$, one finds the value of the internal energy to be about $2 \times 10^{38} \frac{\mathrm{erg}}{\mathrm{gram}}$, where $\rho=7.8 \times 10^{9} \frac{\mathrm{L}}{\mathrm{cc}}, T=.6 \mathrm{Mev}$ and $\bar{Z}=.42$ 
are ued (these values of $p, T$, and $\bar{Z}$ are typical of evolutionary calculations). Again uning (5.15), the internal energy per gram contained in the eloctron gas at $T=.6 \mathrm{Mer}$ minus the value at, $T=0$, impliet there is $4 \times 10^{17} \frac{\mathrm{er}}{\mathrm{mm}}$ in thermal energy svalable for the Fe thermal dimaciation. Since around $1.65 \times 10^{i *}$ ergo of energy is needed to sonvert one gram of iron into one gram of helium, a rough extimate of the amount of iron breakup oeeded to completely exheust the thermal energy in the core is $25 \%$.

The Saha equation for reaction (4) can be written as:

$$
\frac{n_{o}^{13} n_{N}^{4}}{n_{F e}}=\frac{2^{15}}{(56)^{\frac{1}{g_{F e}}}}\left(\frac{m_{B} k_{B} T}{2 \pi h^{2}}\right)^{24} \exp \left(-\frac{125}{T_{M e 0}}\right)
$$

where $g_{F c}$ is the atatistical weight of the iron. Uaing $n_{N}=\frac{1}{15} n_{a}$, and introducing the mass fraciions $X_{\alpha}, X_{N}$, and $X_{F_{e}},(5)$ can be rewritten as:

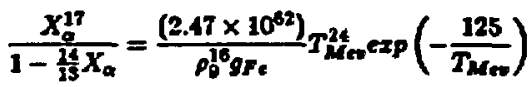

where $X_{N}+X_{\alpha}+X_{F c}=1$ has beer used.

Figure 2 shows the $(\rho, T)$ plane and curves of conatant $X_{0}$. Also shown is the tre:k of the temperature and density of the inner zone found in a numerical caiculation of the collafse of a $15 M_{\odot}$ star, parameterized by time. At these temperatures, $9 \mathrm{~F}$, can be much greater than unity, due to the many excited states of iron. The expreasion used for $g F e$ in constructing figure 2 is taken from Bowers and Wilson (1982b).

The track shown in figure 2 can be understood as follows. Since the iron breakup is endothermic, the energy needed to fuel $\left(4\right.$ ) is lost to pressure aupport ( $\gamma$ drops below $\frac{1}{3}$ and $\left.P \propto \rho^{\gamma}\right)$. In order to try and compensate for the pressure deficit, the atar begins to contract in order to increase the density and temperature and thus reduce the deficit. This, however, leads to more iron thermal disintegration which forces the star to contract further, and thus the nature of the instability becomes apparent. Note, for the evolutionary track shown, the density increases by a factor of 100 while the wemperature only increases by a factor of 4 , reflecting the fact that the major pressure support is electron degeneracy pressure.

The appreciable breakup of helium by thermal disintegration into two protons anc two neutrons requires higher temperatures than reactions (4) at similar densities. In the breakup up of helium, three new particles are produced and $28.3 \mathrm{Mev}$ of energy is required. This gives $9.5 \mathrm{Mev}$ per new particle produced. The breakup of iron to 13 helium nuclei and 1 neutrons produces 16 new particles and requires $125 \mathrm{Mev}$, giving an average of $7.8 \mathrm{Mev}$ per 
new particle produced. The new particles muat have come positive eneray, however unall. Therefore, these is a range in temperature where iron can be dinociated while belium break up is not likely. However, a very amall fraction of free protons will be preasit, due to some belium breakup, which will be important for electron conture.

\section{Eleetron Capture on Protone}

Electrons can be captured on protons in nuclei, or on free protons. This atatement can be translated into the following reactions:

$$
\begin{gathered}
e^{-}+p \Rightarrow n+\nu_{e} \\
e^{-}+N(Z, A) \Rightarrow N^{\bullet}(Z-1, A)+\nu_{c}
\end{gathered}
$$

with (8) followed by:

$$
N^{\bullet}(Z-1, A) \Longrightarrow N(Z-1, A)+\gamma
$$

where $N(Z, A)$ represunts a rucleus with $A$ nucleons and $Z$ protons, and $N^{*}(Z, A)$ indicates an excited state of $N(Z, A)$.

The reaction rate for (7) or (8) can be written using Fermi's Gulden Rule (wouming * given intial state) as:

$$
d \Gamma=\frac{2 \pi}{\hbar}\left(\frac{1}{2} \sum_{\text {spins }}\left|H_{j i}\right|^{2}\right)\left(1-f_{\nu}\right) \frac{d^{a} p_{\nu}}{(2 \pi \hbar)^{3}} \delta\left(E_{\nu}+Q-E_{c}\right)
$$

where $\left(1-f_{\nu}\right)$ is a blocking factor that inhibits $(7)$ or $(8)$ if the final neutrino state is already occupied. $H_{f i}$ is the interaction Hamiltonian, $p_{\nu}$ is the neutrino momentum, $E_{\nu}$ is the neutrino energy, $E_{e}$ is the electron energy, and $Q$ is the difference in energies of the final and initial nuclear states. At the temperatures seen in a supernova collapse, the neutrons and protons or heavy nuclei never become relativistic, so the use of Fermi's golden rule in the non-r ${ }^{\cdot}$ tivistic limit is justified.

Co: applying (10) to (7). At the energies seen in a supernova (i.e. less than 50 $\mathrm{Mev}$ ), the weak interaction can be considered to be a contact interaction, and in the rest frame of the proton, the neutron produced remains almost at rest. Assuming the dipole approximation in calculating $\left|H_{f i}\right|$ (i.e. $\left.\exp \left(\frac{i}{h}\left(\vec{p}_{e}+\overrightarrow{p_{\nu}}\right) \cdot \vec{x}\right) \approx 1\right)$ one sees that $\left|H_{f i}\right|^{2}$ must only depend on weak interaction constants (i.e. no energy dependence, so it is just a number). Equation (10) can be rewritten as:

$$
d \Gamma=\frac{\sigma_{o}}{2}\left(\frac{1+3 \alpha^{2}}{4}\right) c\left(1-j_{\nu}\right)\left(\frac{E_{\nu}}{m_{e} c^{2}}\right)^{2} \delta\left(E_{\nu}+Q-E_{\varepsilon}\right) d E_{\nu}
$$


where $\sigma_{0}=\frac{4 G_{k}^{2} \mathrm{~m}_{2}^{2} n^{2}}{\mathrm{x}^{2}}=1.7 \times 10^{-44} \mathrm{~cm}^{2}, \alpha \approx 1.2$, and $G_{F}$ is the weak interaction constant.

Eariy in the collapere, the neutrinos eacape from the atar and so rob the core of internal ene: gy and pressure suppport. To find the neutrino luminosity from (7), integrate (11) over the initial electron states after fint sultiplying by $E_{\nu}$ and the number of protons. Since $\frac{\mu_{1}}{\mathrm{~s}_{\mathrm{g}} \mathrm{T}} \approx 10$ in the core at the onset of collapee, and $\mu_{\varepsilon} \geq Q \approx 1.4 \mathrm{Mev} \geq m_{\varepsilon} c^{2}=.5 \mathrm{Mev}$, the integration simplifies, and one finds:

$$
L_{\nu_{e}}=\left(4.9 \times 10^{15}\right) X_{P} P\left(\frac{\mu_{e}}{m_{e} c^{2}}\right)^{6} \frac{\mathrm{erg}}{3 \mathrm{~cm}^{3}}
$$

where $X_{P}$ is the mass fraction of protons.

The average energ: of the emitted neutrino can be found using (11). The expression becomes:

$$
<E_{\nu}>=\frac{\int_{Q}^{\mu_{+}} d E_{c} E_{c}^{2}\left(E_{e}-Q\right)^{3}}{\int_{Q}^{\mu_{+}} d E_{e} E_{c}^{2}\left(E_{e}-Q\right)^{2}} \approx \frac{5}{6} \mu_{e}
$$

Now:

$$
\mu_{e}-<E_{\nu}>=\mu_{N}-\mu_{P}=\hat{\mu}
$$

and:

$$
\frac{n_{P}}{n_{N}}=e^{-\frac{n_{0}}{n_{F}}}
$$

Using (12),(13),(14),(15), and $X_{N}=\frac{1}{13} X_{\alpha}$, one finds:

$$
\frac{L_{v_{1}}}{p}=\left(3.8 \times 10^{12}\right) X_{a} \exp \left(-\frac{\mu_{c}}{6 k_{B} T}\right)\left(\frac{\mu_{c}}{m_{c} c^{2}}\right)^{6} \frac{\mathrm{erg}}{g \mathrm{sec}}
$$

Since $X_{\alpha} \leq .2$ during the collapse phase, and $\frac{\mu_{c}}{k_{B} T}$ does not vary much (see figure 2 , and use $\mu_{c} \approx 11.1\left(\rho_{10} \bar{Z}\right)^{\frac{1}{3}}$ Mev) during collapse ( so take $\frac{\mu_{e}}{k_{B} T} \approx 10$ ) to find:

$$
\frac{L_{\nu_{a}}}{\rho} \leq\left(2 \times 10^{11}\right)\left(\frac{\mu_{c}}{m_{e} c^{2}}\right)^{6} \approx\left(2 \times 10^{17}\right) \frac{\operatorname{erg}}{g \sec }
$$

As previously mentioned, there is about $2 \times 10^{15} \frac{\mathrm{mat}}{\mathrm{mam}}$ of internal energy contained in the electron gas at thr start of the collapse. Uaing (17), reaction (7) would exhaust this energy store in about 10 seconds.

Electron capture on nuclei is more complicated due to the fact that the neutron produced must be able to find an available state. Since heavy nuclei such as Fe have more neutrons than protons, part of the energy of the captured electron must go into boosting the proton 
to a higher energy neutron state. Bethe et al. (1979) argue that the daugbter nucleus is excited about $3 \mathrm{Mev}$ sbove a lower enersy atate. Prom a shell model of the nuclews, the proton cannot reach the lowest energy neutron atate available since angular momentum would not be conserved. They bave their andyais on the following reaction:

$$
{ }^{6} \mathrm{Fe}+e^{-} \Longrightarrow \mathrm{Mn}^{*}+\nu_{\mathrm{c}}
$$

which they take as representative of electron capture during collapee.

It should be pointed out that, as electron capture occurs, the typical nucleus has $\frac{z}{A}$ less than $\frac{26}{66}=.46$, the value for iron, and will also begin to acquire neutrons as the high electron chemical potential inhibits neutror decay.

Fuller et al.(1982a,b) and Fuller (1982) have done detailed calculations of electron capture rates in nuelei and find the Bethe et 1 . (1979) estimate of $3 \mathrm{Mev}$ to be good. Also discovered is that when $A-Z \approx 40$, the proton must begin filling another shell that is much higher in energy than the shel! being filled when $A-Z$ is less than 10 . This means the capture rate will be reduced at this point, by about a factor of $\frac{1}{10}$ (private sommunication from Jim Wilson).

Note that Baym et al.(1971) argue that the atomic number $A$, of the average heavy nucleus is given by:

$$
A=194(1-\bar{Z})^{2}\left(1-.236 \rho_{12}^{\frac{1}{3}}\right)^{-1}
$$

and can become large as the density increases towards nuclear density.

A Fermi gas model of a nucleus can be used, along with the recognition that the daughter nucleus is in an excited state of $3 \mathrm{Mev}$ to find an approximate capture rate or neutrino luminosity for (18) (see Bethe et al. (1979) for this type of calculation). An easy way to estimate the luminosity is to use the luminosity for (7), and assume that perhaps $10 \%$ of the protons in each nucleus are energetic enough to capture (i.e. those near the Fermi energy in a statistical model of the nucleus). Equation (12) becomes:

$$
L_{\nu_{*}}^{A}=\left(\frac{.1 Z}{A}\right)\left(4.9 \times 10^{13}\right) \rho\left(\frac{\mu_{e}}{m_{e} c^{2}}\right)^{6} \frac{\operatorname{erg}}{g \sec }
$$

Assuming $\frac{2}{A} \approx .46$ (for iron) and $\mu_{\epsilon} \approx 5 \mathrm{Mev}$, the neutrino luminosity per gram can be found from (19) to be:

$$
\frac{L_{\nu_{0}}^{A}}{\rho} \approx\left(2 \times 10^{18}\right) \frac{e r g}{g \sec }
$$


Since the internal energy is about $2 \times 10^{18} \frac{\mathrm{me}}{\mathrm{mam}}$, the reaction (B) cen deplete this internal energy on 1 second time scales.

Note that electron capture, unlike iron thermal disintegration, is not an equilibrium process, and thus can change the entropy per baryon. The neutrino can eacape from the system, and lower the entropy, but since capture ratea lag behind equilibrium, entropy can increase (i.e. bulk viscosity). In practice, the entropy remains almost urchanged.

This section can be concluded by recognizing that when pressure support is lost through iron thermal disintegration and electron capture on protons, the iron core must collapse on a freefall timescale. An estimate of this collapse time is thus:

$$
t_{/ /} \approx \frac{1}{\sqrt{G \bar{\rho}}} \approx .1 \mathrm{sec} .
$$

Actually, since pressure support is not completely abeent, the time for collapse is several times longer.

Also a burst of neutrinos is producei when the collapse accelerstes, as the election capture on nuclei reduces the value of $\bar{Z}$, the number of matter electrons per baryon, to about .33 . The neutrinos cease escaping freely as the density reaches values where the neutrino mean free path is on the order of the size of the radius. Using equation (11), an estimate of the mean free path of a neutrino is:

$$
\frac{1}{\lambda_{\nu}}=\frac{\sigma \rho}{m_{B}} \approx \sigma_{0}\left(\frac{E_{\nu}}{m_{e} c^{2}}\right)^{2} \frac{\rho}{m_{B}}
$$

Therefore $\lambda_{\nu} \approx 10^{6} \mathrm{~cm}$ when $E_{\nu} \approx 3 k_{B} T \approx 10 \mathrm{Mev}$, and $\rho \approx 2.5 \times 10^{13} \frac{\ell}{e c}$.

Figure 3 shows the mass point trajectories of the iron core during the collapse phase, and the bounce, which is the subject of the gext section.

\section{BOUNCE AND SHOCK FORMATION}

Figure 4 shows a sequence of infall velocity curves, parameterized by the central density, and covering a .001 second span in time. The last curve, with $\rho=1.3 \times 10^{14} \frac{\ell}{e \varepsilon}$, is just before bounce and shock formation.

Evolutionary calculations give a value for $s$, the entropy per barjon in units of Boltzmann's constant, around 1 at the start of the collapse. Bethe et al. (1979) point out that $s$ cannot change appreciably during collapse. This implies that most nucleons stay inside the heavy nuclei, and thus the nuclear contribution to the pressure is small during collapse. In fact, most nucleons stay inside he heavy nuclei until the heavy nuclei touch and a uniform 
sea of nuclear matter forms. At densities near but atill iess ihan nuclear denuity, the nuclear pressure is negative due to the attractive nuclear ferce. At densities above nuclear density, the nuclear force becomes repulsive and the nuclear pressure will dominate all other sources of pressure.

The structure of the curves in figure 4 can be undentood a follown. By the above discussion, the major pressure support during collapse is degenerate electrons, which gives $P=K \rho^{\gamma} \approx K \rho^{\frac{4}{3}}$ (as previously noted, $\gamma$ is alightly lese than $\frac{1}{3}$ due to iron thermal disintegration and electron capture). $A \gamma=\frac{1}{3}$ gas is neutrally stable, and there is no natural length scale that can be constructed from $K, G$, and $M$. What happena in that material inside the sonic point (i.e. the point in the star where the sound apeed equals the infall velocity) can stay in communication and collapse homologously in what are succeasive $n=3$ polytrope-like configurations. The material outside the sonic radius collapaea in quasi-free fall. Considerable analytic work has been done on this phase of collopse by Goldreich and Weber (1980) and Yahil and Lattime: (1982). The results of this work produced a prediction for the size of the homologous core, which turns out to be very close to the Chandrasekhar mass. Another prediction is that the material outside the homolcgous core has a density that varies as $\frac{1}{R^{3}}$.

Since the value of $\bar{Z}$ is now around .33 due to the electron capture, $M_{H}$, the mass of the homologous core is about:

$$
M_{H} \approx M_{C H}=5.76 \vec{Z}^{2} M_{\odot}=.63 M_{\odot}
$$

In realistic calculations, $M_{H}$ is found to be about $.55 M_{\odot}$. It is smaller than the predicted value due to the negative nuclear pressure at densities near but still less than nuclear. The prediction that the density varies as $\frac{1}{R^{j}}$ is seen to be satisfied in numerical simulations.

When the central density climbs to $1.9 \times 10^{14 \frac{q}{c c}}$, nuclear density has been reached (since $\bar{Z} \approx .33$, nuclear density is less than $2.7 \times 10^{14} \frac{\ell}{\varepsilon}$, the value for $\bar{Z}=.5$ ). At this point $\gamma$ begins to rise above $\frac{4}{3}$ due to the near incompressible nature of nuclear matter above nuclear densities, and the collapse halts. The experimental value of $K_{N}=9 m_{B} \frac{d P}{d_{p}}$, the nuclear compressibility modulus, is found to be $220 \mathrm{Mev}$ (see Blaziot et al. (1976)). (Note, at densities many times nuclear density, $\gamma$ must be less than 2 , if the condition that the sound speed is less than the speed of light is fullfilled (see Lightman et al. (1975)).)

Brown et al. (1982) argue that pressure waves travel from the halted central regions 
outward, carrying information of the impending bounce to the still falling material at larger radii. The preseure waves cannot pase far beyond the wonic point without a ahock wave forming. The homologous core bounces as a unit and the rebound launches the shock nave into the infalling material. The thock weakens a heavy nuclei passing through the shock are dissociated into nestrons and protons. When the ahock moves through and beyond the neutrinosphere, the post shocked heated material radiates neutrinos that chn escape the star and further weaken the shock.

The question of the shock promptly moving outward is a thorny one. Baron, Cooperstein and Kahan (1985) exploit the uncertainty in the supra-nuclear equation of atate and find that a soft enough supra-nuclear equatios of state (i.e. taking $K_{N}$, the compressibility modulus to be around 120-140 Mev) and a slightly stiffer mb-nuclear equation of atate (thus lessening the effect of the attractive part of the nuclear presoure, and increasiug the size of the homolognus core) produces a shock initially energetic enough to reach a radius where the energy losses to neutrinos and the dissociation of heavy nuclei have become small. These losses become small when the shock weakens to the point where it is unable to dissociate nuclei, and if the pust shock temperature ia small enough so that neutrino production falls. The softer equation of state allows a deeper bounce and s large homologous core redures the amount of material the shock must dissociate in its exit from the core.

The equation of state we are using has a compressiblilty modulus more in line with the laboratory value of $220 \mathrm{Mev}$ found on messurements made with such nuclei as ${ }^{208} \mathrm{~Pb}$ which has $\bar{Z}=.394$ (see Blaziot et al. (1976)). Using $K_{N}=220 \mathrm{Mev}$ prooiuces a much stiffer equation of state than using a value around $140 \mathrm{Mev}$. Consequently the rebound is not as helpful in launching an energetic shock, as found by Baron, Cooperstein and Kahana (1985).

In the following, the bounce meshanism is assumed to fail in producing in explosion. Figure 5 extends figure 3 to later times. As can be seen, an explosion occurs, and a protoneutron star of baryon mass $1.6 M_{\odot}$ is left as a remnant. The next section discusses the physics important to the late time explosion.

\section{LATE TIME SUPERNOVA}

An important feature of figure 5 is that the region outside the neutrinosphere is composed of neutrons and protons. The existence of these free nucleons is due to the dissociation of heavier elements by the shock. 
The presence of neutrons and protons outside the neutrinosphere in important because they are able to absorb and emit neutrino and antineutrino by the following reactions:

$$
\begin{aligned}
& n+\nu_{c} \Longleftrightarrow p+e^{-} \\
& p+\overline{\nu_{c}} \Longleftrightarrow n+e^{+}
\end{aligned}
$$

The gravitational binding energy of a cold neutron atar is about .1Mc. Uaing $M=1.6 \mathrm{M}_{\odot}$, one finds about $3 \times 10^{53}$ ergs of energy that must be radiated away (mainly in zeutrinos of all types). The possibility exists that nucleons outside the neutrinosphere may absorb energy from the electron neutrinos and electron antineutrinos produced by. the forming neutron star, and so heat the material under the shock. The heating increases the material pressure behind the shock, wh:ch helps the shock to move outward.

An estimate of the comparition of the star behind the shock can be made independent of a computer simulation, us ing an energy conservation argument. Assuming iron is dissociated to neutrons and protons by the shock, the following equation holds:

$$
\frac{G M m_{P e}}{R} \approx\left(8 M e v+\frac{3}{2} T_{M e v}\right)(56)
$$

or, taking $M=1.6 M_{\odot}$ :

$$
\frac{22}{R_{7}}=8+\frac{3}{2} T_{M e v}
$$

where $R_{7}=10^{-7} \times R$ with $R$ in cm. Assuming iron dissociates to helium (needing approximately $2 \mathrm{Mev}$ per nucleon) one finds:

$$
\frac{22}{R_{7}}=2+\frac{3}{2} T_{M e v}
$$

Finally, assuming the shock is not strong enough to dissociate the iron, results in:

$$
\frac{22}{R_{7}}=\frac{3}{2} T_{M e v}
$$

In figure 6 equations (23),(24), and (25) are graphed, and the regions where the shock can dissociate the material into baryons, helium, or no dissociation can be seen. The actual temperature would be an interpolation of the disjointed segments. 
This simple construction reproduces the general composition features wen in figure 5 , after the bounce and ahock formation. The temperature profile in also roughly in agreement vith the numerical simulations.

The net rate of heating of the otellar material outside the neutrinouphere can be catimated. In the region near $\boldsymbol{R}=10^{7} \mathrm{~cm}$, at late times, the densities are more aimilar to the outer regions of the original iron white dwarf core, and are around $10^{2} \frac{\ell}{e}$. From foure 6 , temperatures are 1-2 Mev. In this region, electrons are relativintic and quani-degenerate (i.e. $\frac{\mu_{B}}{k_{B} T} \approx 1.7$ ). Equations $(20)$ and $(21)$ will be written as:

$$
b+1 \Longleftrightarrow b+\nu
$$

where $\frac{\mu_{s}}{k_{g} T} \approx 0$ is assumed ( this implies that the number density of the electrons is identical to the number density of the positrons). Also, the mass difference between the neutron and proton, and the type of neutrino (particle or anti-particle) will be ignored.

The matter cooling rate per baryon due to $b+l \Longrightarrow b+u$ can be written using (11) as:

$$
\dot{E}_{\text {cool }}=\int E_{\nu} \frac{E_{l}^{2}}{\pi^{2}(h c)^{3}} f l d E_{l} d \Gamma
$$

Assuming:

$$
f=\frac{1}{\exp \frac{E_{l}}{k_{B} T}+1}
$$

the integral (with the neglect of blocking factors) can be done to find:

$$
\dot{E}_{\text {cood }}=8.1 \sigma_{0} c\left(\frac{m_{e} c}{\hbar}\right)^{s} m_{e} c^{2}\left(\frac{k_{B} T}{m_{e} c^{2}}\right)^{6}
$$

The matter heating rate per baryon due to $b+\nu l \Longrightarrow b+l$ is calculated in a similar manner as fullows:

$$
\dot{E}_{\text {heat }}=\int E_{\nu} \frac{E_{\nu}^{2}}{B \pi^{3}(h c)^{3}} f_{\nu} d E_{\nu} d \Omega d \Gamma^{*}
$$

where:

$$
d \Gamma^{*}=\sigma_{o} c\left(\frac{1+3 \alpha^{2}}{4}\right)\left(1-f_{i}\right)\left(\frac{E_{l}}{m_{e} c^{2}}\right)^{2} \delta\left(E_{l}-E_{\nu_{c}}\right)
$$

Figure (7) illustrates some notation, and shows the physical picture that is being assumed in the derivation. 
Since the regijn of interest is outside the neutrinosphere, the neutrino distribution function, $f_{\nu}$, is assumed to remain constant along a beam, and have that value characteristic of neutrinos in equilibrium with the mutter at temperature $T_{a}$.

The angular intergral in (30) can be approximately done giving:

$$
\dot{E}_{\text {heat } \approx} x\left(\frac{R_{p}}{R}\right)^{2} \int \frac{E_{\nu}^{s}}{\mathrm{Bx}^{3}(h c)^{3}} f_{\nu} d E_{\nu} d \mathrm{~T}^{*}
$$

Now completing the integrals in (32) using:

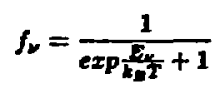

one finds:

$$
\dot{E}_{\text {heat }}=8.1 \sigma_{0} c\left(\frac{m_{c} c}{\hbar}\right)^{3} m_{c} c^{2}\left(\frac{R_{p q}}{2 R}\right)^{2}\left(\frac{k_{B} T_{R}}{m_{c} c^{2}}\right)^{6}
$$

Measuring temperatures in Mev, the net heating rate per baryon becomes:

$$
\dot{E}_{n e t}=\left(3.3 \times 10^{-6}\right)\left(\left(\frac{R_{a p}}{2 R}\right)^{2} T_{m}^{6}-T^{6}\right) \frac{\text { erg }}{\text { baryon sec }}
$$

where temperatures are measured in Mev. For an alternate way of obtaining (34), zee Bethe and Wilson(1985).

$\dot{E}_{\text {net }}$ is positive provided:

$$
\frac{R_{p p}}{2 R} \geq\left(\frac{T}{T_{p p}}\right)^{3}
$$

Using $T_{s p} \approx 5 \mathrm{Mev}, R_{s p} \approx 3 \times 10^{6} \mathrm{~cm}$, one finds:

$$
R \approx 2 \times 10^{7} \mathrm{~cm}
$$

The gravitational potential energy per baryon at $R=2 \times 10^{7} \mathrm{~cm}$ is:

$$
E_{\text {grau }}=\frac{G M m_{B}}{R} \approx 1.8 \times 10^{-5} \frac{\mathrm{erg}}{\text { baryon }}
$$

where $M=1.6 M_{\odot}$ has been taken.

Equations (34) and (35) define a timescale for the net heating to overcome the gravitational binding as:

$$
t_{E x p}=\frac{E_{\text {grav }}}{\dot{E}_{\text {net }}}=.03 \mathrm{sec}
$$


where $T_{s p}=5 \mathrm{Mer}, T=1 \mathrm{Mev}, R_{s}=3 \times 10^{6} \mathrm{~cm}$, and $R=3 \times 10^{7} \mathrm{~cm}$ heve been taken in (34). These are typical values seen in a collape calculation.

Assuming an explosion does occur, by neutrino heating and the subeequent revival of the shock, the density is the region between the shock and the proto-neutron star will drop as the envelope begins to separate from the remnant. The density is dominated by neutrons and protons, formally the major source of interaction for the neutrinos. As the density falls, elsetron-positron pairs become the the major opacity source for neutrino interaction. As will be seen in Chapter IV, the opacity for nestrino intcraction with electrons can be written as:

$$
k_{c}^{*}=(\text { const }) \frac{\sigma_{0}}{m_{B}} \frac{E_{\mu} k_{B} T}{\left(m_{e} c^{2}\right)^{2}}
$$

in regions of high temperature and low density (i.e. $\frac{k_{n} r}{\mu_{n}} \geq 1$ ). It is possible for the material in the bubble behind the shock to interact with the core neutrinos and absorb energy through scattering with the electron-positron pairs.

Thus the blojle region can be heated by the neutrinos, and so prevent it: = temperature from rapidly falling, ss it wouid without some heating mechanism, when the shock moves outward during the explosion.

Photons and electron-positron pairs wil' provide pressure support (i.e. $P=\frac{11}{12} a T^{4}$, where $a=\frac{\sigma_{n}}{c}$, with $\sigma_{B}$ the Stephan-Boltzmann constant), even though the density (dominated by the baryons) is falling.

Figure 8 shows the results of the numerical computation for a $25 M_{\odot}$ progenitor star. Here, another phenomena is clearly seen. The neutrino luminosity can oscillate in time, as well as the mass accretınn rate. Chapter VI will report preliminary results in the study of this neutrino oscillator.

The last subject to discuss in Chapter II is the possible effects of magnetic fields or rotation. The median rotational period, ${ }$ rot, of the observed pulsars is about $.67 \mathrm{sec}$ ( $\mathrm{see}$ Shapiro and Teukolsky (1983)). The shortest period is $1.558 \mathrm{~ms}$ (PSR 1937+214; Backer et al. (1982)). The vibrational period, $f_{v i b}$ of a neutron star is about $.5 \mathrm{~ms}$ (i.e. ivib $\approx \sqrt{\frac{A^{3}}{G M}}$ ). $\frac{T_{\text {sib }}}{T_{\text {rot }}} \approx .3$, even in the most extreme example, hence the dynamic effects of rotation are negligible, and must even have been of less importance in the formation of a neutron star.

Since $B \approx 10^{12}$ gauss for the majority of observed pulsars, $P_{B}=\frac{1}{8 \pi} B^{2} \approx 4 \times 10^{22} \mathrm{cgs}$. This is completely nagligible to matter pressures seen in a collapse. At the onset of collapse, 
the pressure in degenerate electrone in approximately $4 \times 10^{27} \mathrm{cs}, 0$ can be wea using equation (5.15) and the fact that the electron promure is about $f$ the eloctron enerdy denaity. During the subsequent evolution, the material preanure reachea moch bigher vilues.

Therefore, for the majority of atars, apherica' collapec calculations must be able to explain the supernova explosions.

A numerical calculation of a supernore including magnetic field and rotation was done by LeBlanc and Wilson (1970) and later repeated witt a new equation of atale and neutrino physies by Syribalisty (1984). Both of these calculations found, for extreme values of magnetic field and rotation, a jet of obout $.07 M_{0}$ in seutronized matter excapes along the rotation axis. This may be a posuible aite for the froduction of the $r$ process nuclei, However, only a small fraction of excepitional supernove are needed to sccount for the $r$ process elements if neutrun jets are produced. These papers concerned the early times of a supurnuva (i.e. late times supernovs were not investigated), and the reader is referred to them for more quantitative details of the effecta of magnetic field and rolation on the evolution of a supernova. 


\section{Chapter II Reference:}

Backer,D.C.,Kulkarni,S.R.,Heiles,C.,Davis,M.M and Goss, W.M. 1982,

Nature, $\mathbf{3 0 0 , 6 1 5 .}$

Baron, E., Cooperatein, J., Kahana, S. 1985, Phys. Rev. Lettr., r55, 126.

Baym, G., Bethe, H.A. and Petbick, C.J. 19?1, Nucl. Pbys., A175, 225.

Bethe, H.A., Brown, G.E., Applegate, J. and Lattimer, J. 1979, Nucl. Pbys, A324, 187.

Bethe, H.A. and Wilson, J.R. 1985, Ap. J., v295,14.

Blaizot, J.P., Gogny, D. and Grammaticos, B. 1976, Nucl. Pbys. A265, 315.

Brown, G.F., Bethe, H.A.and Baym, G. 1982, Nucl. Phys., A375, 481.

Clayton, D. 1968, “Principles of Stellar Evolution and Nucizusynthesis”, (McGraw-

Hill:New York), Chapter 7.

Goldreich, P. and Weber, S.V. 1980, Ap. J., v238, 991.

Fuller, G.M. 1982 , Ap. J., v252, 741.

Fuller, G.M., Fowler, W.A. and Newman, M.J. 1982a, Ap. J. Suppl. Ser., r48, 279.

—. $1982 b$, ap. J., v252, 715.

Le Blane, J.M. and Wilson, J.R. 1970, Ap. J., v161, S41.

Lightman, A.P., Press, W.H., Price, R.H. and Teukolsky, S.A. 1975, "Problem Book in

Relativity and Gravitation", (Princeton University Press:Princeton), Chapter 5.

Shapiro, L. and Teukolsky, S.A. 1983 "Black Holrs, White Dwarfs, and Neutron Stars", (John Wiley and Sons, Inc. New York), Chapter 10.

Shu, F. 1982, "The Physical Universe", (University Science Books: Mill Valley, Ca.),

Chapter 9.

Symbalisty, E. 1984 , Ap. J. v285, 729.

Weaver, T.A. and Woosley, S.E. 1980, Ninth Texas Symp. Rel. Ap., Ann. N.Y. Acad. Sci., v336, 335.

Yahil, A. and Lattimer, J.M. 1982 in "Supernova:A Survey of Current Research" ed.

M.J. Rees and R.J. Stoneham, (Reidel, Dordrecht:Holland) 


\section{Chapter II Figure Caption:}

Fig. 1 Hertzoprung-Runell Disgram edapted from Shu (1902).

Fig. 2 Curve of constant Helium man frection on $2(6, T)$ plase.

Fig. 3 Collapee and bounce of a 15 solar man star. The aymbol B indicates free baryons.

Fig. 4 Velocity versus redius for the 15 wolar mas atar during the final .001 eecond before bounce.

Fig. 5 Late time behavior of the 15 oler mene ator. This figure continues foure 3.

Fig. 6 Approximate temperature and composition profle behind the shock.

Fig. 7 Illustration of the phyoical conditions important to underatanding the late time neutrino heating mechanism.

Fig. 8 A late time supernova of a 25 wolar mace progenitor. The phenomena of cecillations in neutrino luminosity and mase accretion rate is clearly ceen. 


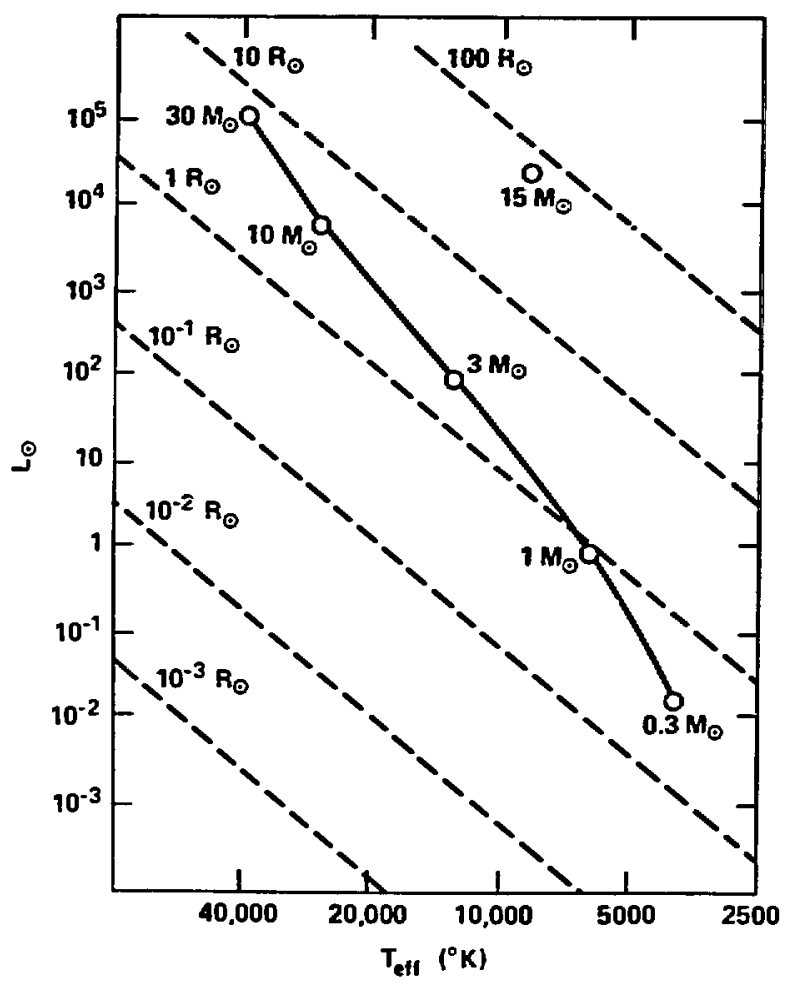

FI GURE 1 


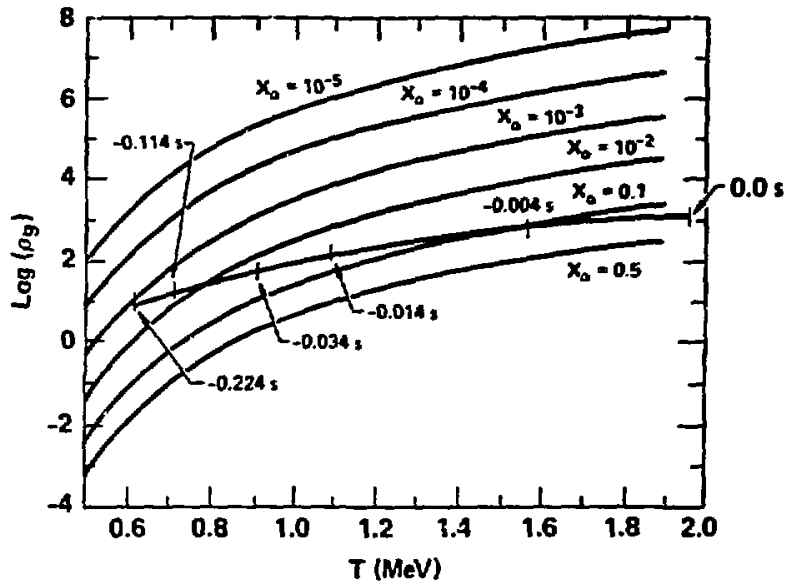

FIGURE 2 


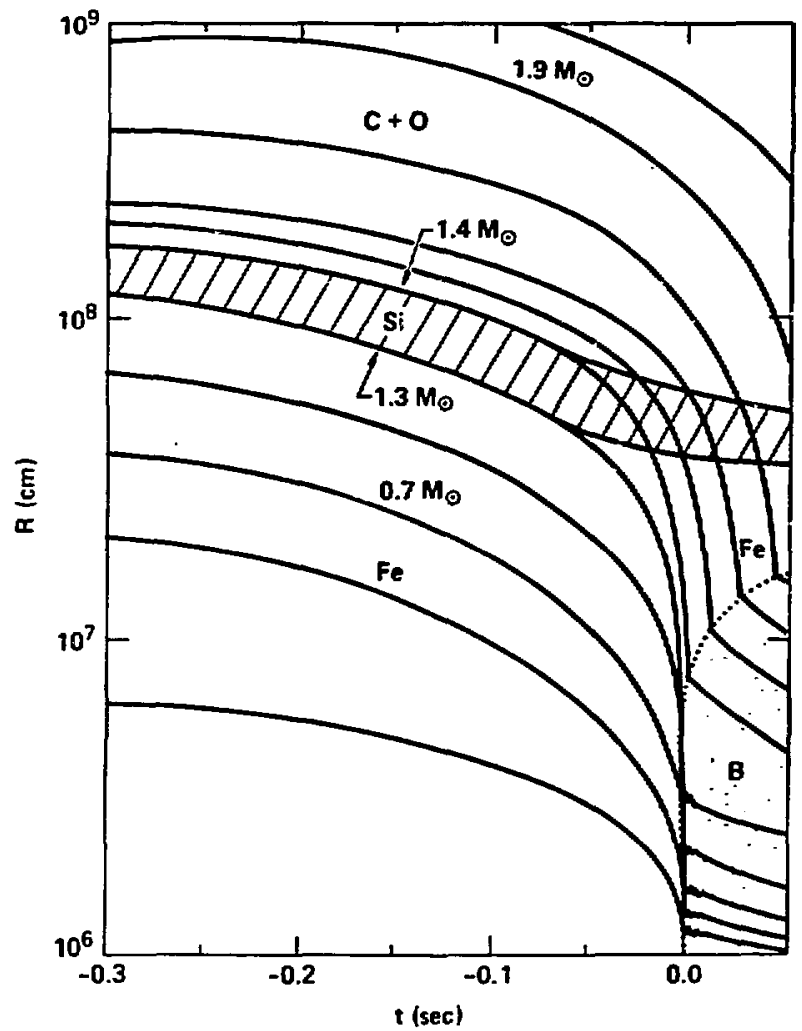

FI GURE 3 


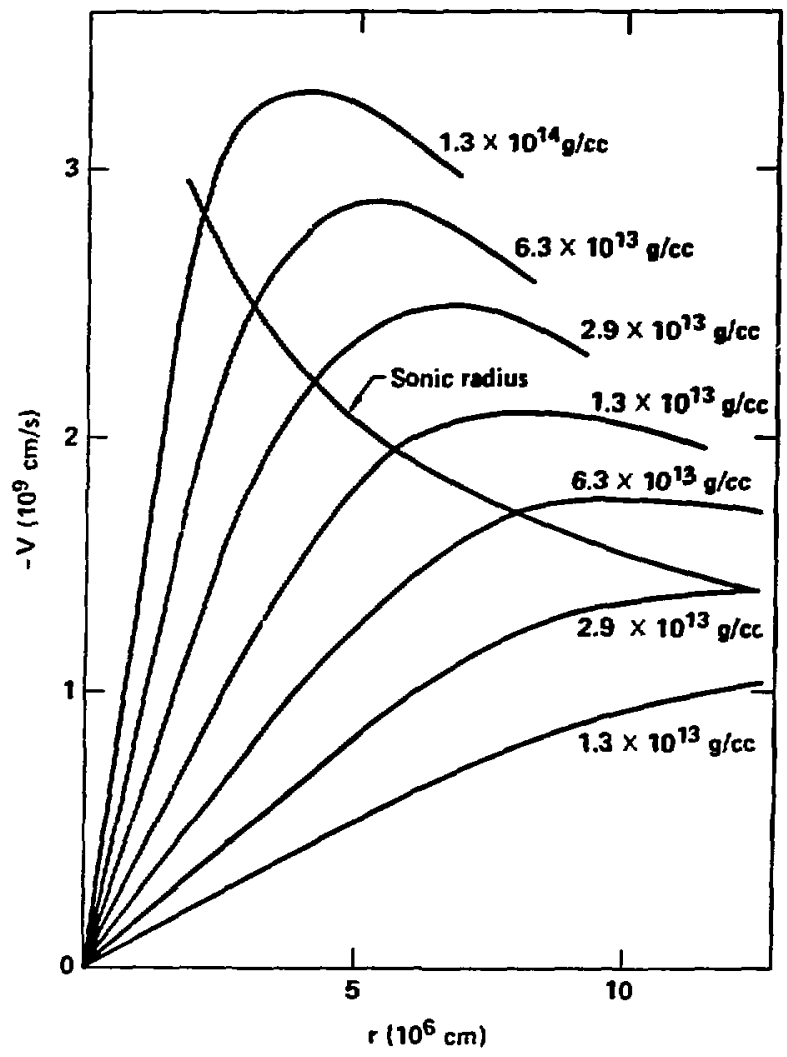

FI GURE 4 


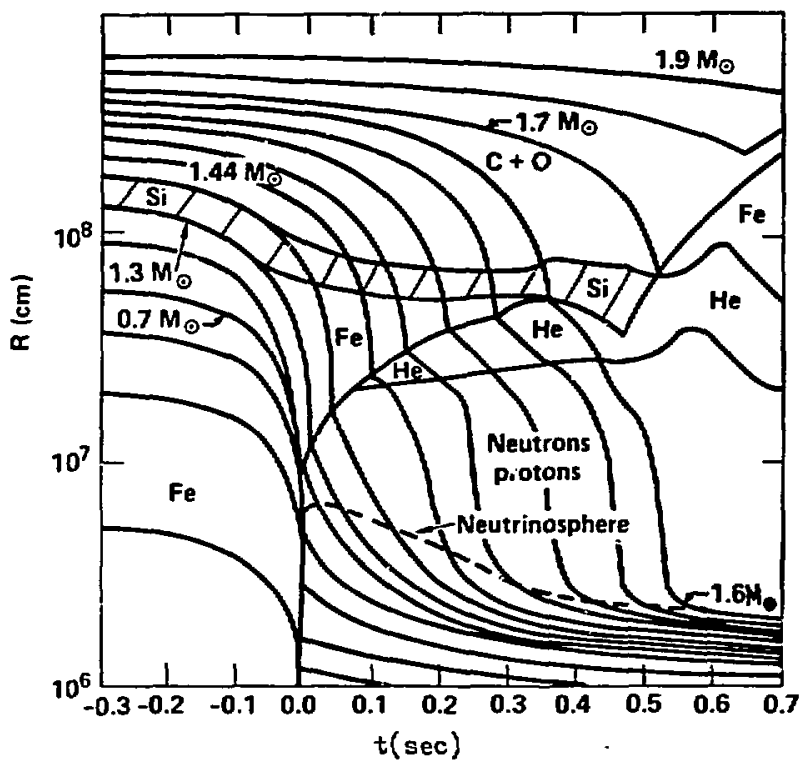

FI RURE 5 


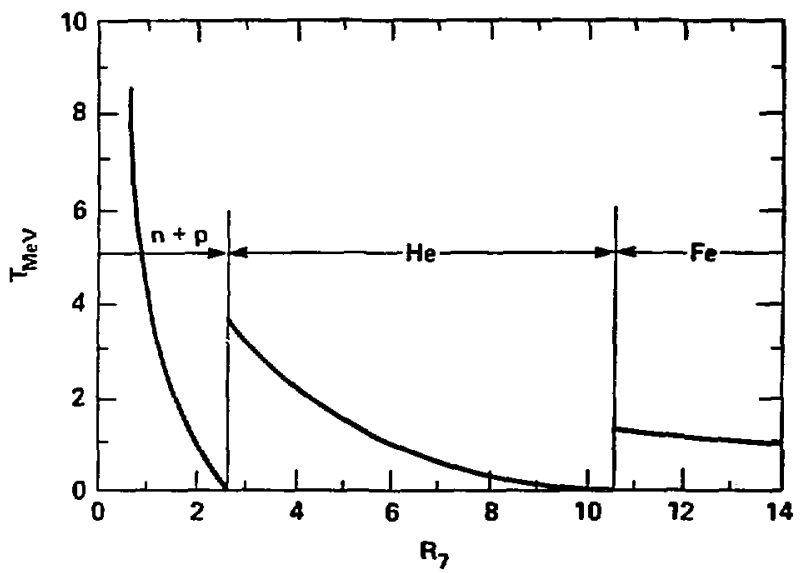

FIGUE 6 


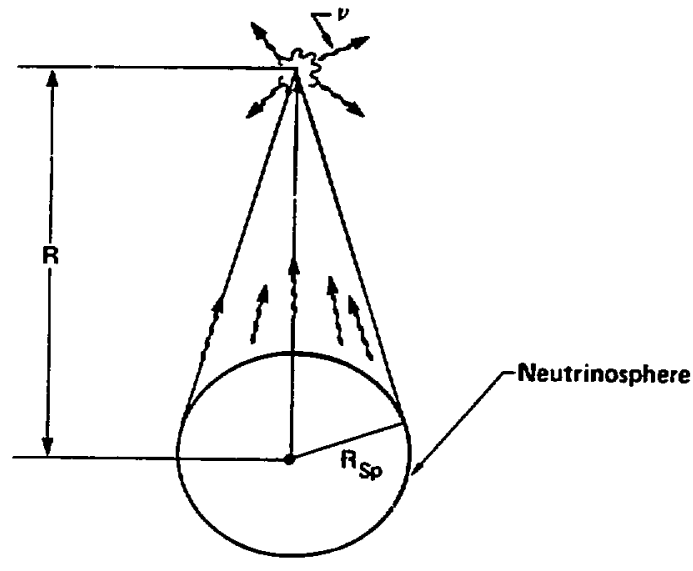

F1 Gงละ 7 


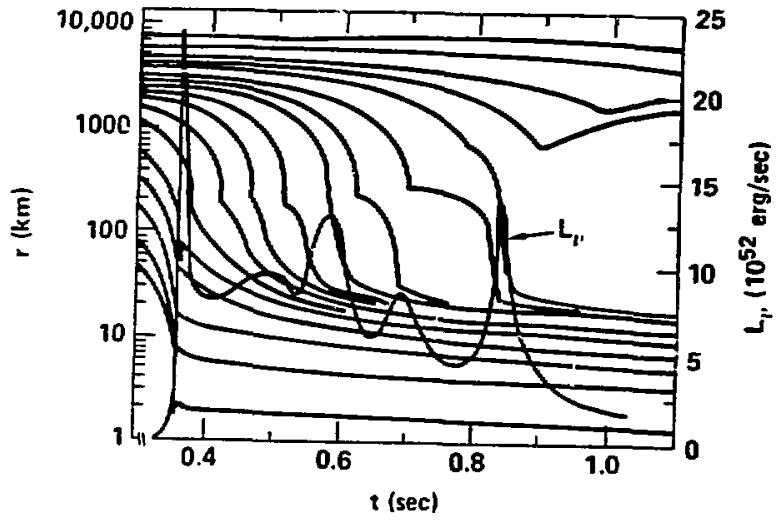

FI GIRE 8 


\section{Chapter III}

\section{EQUATIONS TO MODEL A SUPERNOVA}

\section{INTRODUCTION}

The supernova code 1 inherited is deacribed in Bowers and Wilson (1982a,b), herearter denoted by BW. In that paper, the equations and numerical methods used were discussed. This code approximated some aspects of general relativity (i.e. the force equation is correct in the static limit).

It has long been known that taking into account the full hydrodynamic effects of general relativity does make a difference in some details of the model supernove evolution (see May and White(1967), Wilson(1971)). One difference is that a deeper bounce is found with general relativity than with Newtonian physics. However, the inclusion of general relativity is not the determining factor in producing supernova explosions. (However, see Baron, Cooperstein, and Kahana(1985) for wome recent work un the combined effects of general relativistic hydrodynamics and various parameterizations of the high density equation of state).

As mentioned above, the supernova model described in BW has an enhanced gravitational force (due to inclusion of static general relativity effeets), that gives a deep bounce, and therefore larger possible infall velocities. An observer far away from the star (and therefore not significantly influenced by the gravitational field produced by the star) may ask if special relativistic effects are important in a supernova collapse.

The question can be answered by looking at a computer simulation (or, see below for an estimate without appealing to computer output). The $v / c$ ratio can become as large as .3: however, at any time, no more than than $0.01 M_{\odot}$ achieves such high velocities. During most of the evolution, $.2 c$ is a more typical peak infall velocity. Special relativistic effects can therefore be estimated to be on the order of $4 \%$ (i.e. $\left.(v / c)^{2} \leq .04\right)$.

In the neutrino transport used by $\mathrm{BW}$, some order $v / c$ effects were taken into account, but the gravitational redshift was not. From computer calculations, it is found that at bounce, the redshift, $\mathrm{z}$, is .25 at the center of the star, rising to .55 at the end of the simulation (about 1 second after bounce). However, at the neutrinosphere, $z=.05$ at bounce, rising to $z=.20$ at the end of the same calculation. Since, inside the neutrinosphere, neutrinos are trapped, $\mathbf{z}$ at the neutrirosphere radius is the important number to consider in 
estimating the effect of redshift on a late time supernova.

As discussed in Chapter II, the hearing rate of the matter behind the whock due to the neutrinos exiting the neutrinosphere is given by:

$$
\dot{E}(R)=k_{\mathrm{u}}\left(\epsilon_{\nu}(R), T\right) \frac{L(R)}{4 \pi R^{2}}
$$

where $L(R)$ is the neutrino luminosity at radiua $R$.

In Chapter II it was noted that the abeorbtive opacity in the temperature and density range found behind the shock can be expreseed as:

$$
k_{\mathrm{a}}=a_{1} \epsilon_{\nu}^{2}+a_{2} \epsilon_{\nu} T
$$

where $a_{1}$ and $a_{2}$ derend on the composition.

In terms of values at the neutrinosphere radius, $R_{p p}$, the luminosity at infinity can be expressed as:

$$
L(\infty)=\frac{L\left(R_{0 p}\right)}{(1+z)^{2}}
$$

and the energy of a neutrinos emitted at the neutrinosphere and observed at infinity is:

$$
\epsilon_{\nu}(\infty)=\frac{\epsilon_{\nu}\left(R_{p p}\right)}{(1+x)}
$$

Since the neutrinosphere is defined to be the radius where the optical depth to infinity is $2 / 3$, there is still a probability for a neutrino to interact outside the neutrinosphere (this fact, of course, is of fundamental importance to late time supernova). For a neutrino observed at infinity, and emitted at the neutrinosphere:

$$
z \approx \frac{G M}{R_{s p} c^{2}} \leq .2
$$

if $\mathrm{M}=1.4 M_{\odot}$ and $R_{o p}=10 \mathrm{~km}$ are taken (see the value of $z$ quoted on the first page of this chapter, for $\mathbf{z}$ at the end of a calculation).

Taking into account that fact that a neutrino absorbed before.escaping feels only a fraction of the redshift at infinity, and that the region important for absorbtion is typically a few times the neutrinosphere radius, a rough estimate of the average redshift at absorbtion is:

$$
<z>=\frac{2}{3} z
$$


(this would correspond to emission at $R_{m}$ and aboorbtion at $3 R_{m}$, a radius chosen as representative for alsorbtion.) Using < >>, (1) can be rewritten a:

$$
\dot{E}(R)=k_{\varepsilon}\left(\frac{\epsilon_{\nu}}{1+<z>}, T\right) \frac{L\left(n_{p p}\right)}{4 \pi R_{\rho p}^{2}}\left(\frac{1}{1+<z>}\right)^{2}
$$

Since $k_{a}$ has both a quadratic and linear dependence on $\epsilon_{\nu}$, the effect of the redshift on tha heating rate can be written as:

$$
\dot{E}(R,<z>)=\left(\frac{1}{1+<z>}\right)^{n} \dot{E}(R, 0)
$$

with $n=3$ or 4, depending on which term dominates the abeorbtive opacity. Assuming $n=4$, $<z>=.13$, the effect of the redshift is to drop the heating rate by $10 \%$ below the value found without redshifting the neutrinos.

From the discussion so far, it is clear that the most important physical process neglected (as far as late time supernova are concerned) is the gravitational redshift of the neutrinos exiting the neutrinosphere.

Another effect of importance (for late time supernova) whicn could increase the neutrino luminosity exiting the core is convection (material mixing would dredge up trapped neutrinos from below the neutrinosphere and allow them to escape). This topic will be the concern of Chapter $\mathrm{V}$.

Originally, a completely new computer code was planned, and Appendix $\boldsymbol{A}$ contains two complete sets of fully general relativistic equations, each of which could be used for such a cude (see below). However, new stellar input models (provided by Stanford Woosley) were becoming available at the time a new code was being planned. This led to a change in direction of research, as new codes take approximately threc years of development and debugging, and we felt that is was important to run the new models as soon as possible. Therefore, of the defects mentioned above, the redshift of the neutrinos was felt to be the most important to remedy in some fashion, snd this could be done without too drantic a change to the present code. In this chapter, the approximate equations now used in the supernova code will be presented. Some attempt will be made to justify any approximation that is made.

Two complete sets of equations are given in Appendix A, one is more appropiate for an Eulerian (fixed coordinate system) solution of the equations, the other is appropiate for a Lagrangian (coodinates tied to matter) solution of the equations. The supernova code I 
inherited uses a Lagrangian system of coordinates, and the equations to be found in this chapter will atart from the Lagrangian equations in Appendix A.

\section{HYDRODYNAMIC ACCELERATION}

The following five equations are repeated from Appendix $A$ :

$$
\begin{aligned}
& \frac{1}{a} \frac{\partial U}{\partial t}=-\frac{4 \pi R^{2} \Gamma}{\left(1+\frac{\operatorname{lom}+P_{m}}{m^{2}}\right)}\left(\frac{\partial P_{L \alpha}}{\partial m}+\frac{2 H}{R} \frac{\partial R}{\partial m}+\frac{1}{c^{2}} \frac{\partial}{\partial t}\left(\frac{\bar{G} b}{a}\right)\right) \\
& -\frac{G M}{R^{2}}\left(1+\frac{4 \pi P_{10 t} R^{2}}{M c^{2}}\right) \\
& M=4 \pi \int_{0}^{R}\left(\rho+\frac{e_{\text {ef }}}{e^{2}}+\frac{\tilde{G} U}{c^{\top} \Gamma}\right) R^{2} d R \\
& \Gamma=\left(1+\frac{U^{2}}{c^{2}}-\frac{2 G M}{R c^{2}}\right)^{\frac{1}{3}}=\frac{1}{b} \frac{\partial R}{\partial m} \\
& b=\frac{1}{4 \pi R^{2} p} \\
& U=\frac{1}{a} \frac{\partial R}{\partial t}
\end{aligned}
$$

where $e_{t o t}=\tilde{\epsilon}+e, P_{t o t}=\tilde{P}+P, H=\frac{1}{2}(\tilde{c}-3 \bar{P})$ and:

$$
\begin{gathered}
e=\text { matter energy density } \\
P=\text { matter pressure } \\
\tilde{e}=\text { neutrino energy density } \\
\tilde{P}=\text { neutrino pressure } \\
\tilde{G}=\text { neutrino energy flux }
\end{gathered}
$$

As mentioned in Appendix $A, \Gamma$ is a relativistic generalization of a special relativistic gamma, and $U \approx \Gamma v$ is the radial component of the matter 4 velocity as measured by an Eulerian observer. Using $v, \Gamma$ can be rewritten as:

$$
\Gamma=\frac{1}{\sqrt{ }\left(1-\left(\frac{\mathrm{v}}{\mathrm{c}}\right)^{2}\right)}\left(1-\frac{2 G M}{R c^{2}}\right)^{\frac{1}{2}}
$$


Now, changing derivatives with respect to $m$ into derivatives with respect to $R$ (uing equation (A.40)), and replacing $U$ with $\Gamma r$, equation (A.44) becomes:

$$
\begin{aligned}
& \left(1+\frac{c_{t+1}+P_{t \alpha}}{\mu^{2}}\right) \cdot\left(\frac{1}{a} \frac{\partial v}{\partial t}+\frac{\partial \ln \Gamma}{\partial t}\right)
\end{aligned}
$$

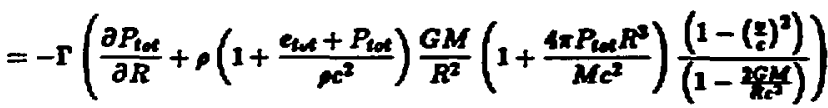

$$
\begin{aligned}
& -\frac{1}{c^{2} b} \frac{a}{\partial t}\left(\frac{\bar{G} b}{a}\right)-\frac{2 \mathrm{r} H}{R}
\end{aligned}
$$

An estimate of $\bar{P} / P$ can be made. When the relativistic corrections in (2) are important (in the central part of the atar), the core will have bounced (after seaching nuclear densities) and the neutrinos will be trapped, and in equilibrium with the matter. Since botk clectrona and neutrinos are relativistic degenerate fermions in the central regions (of the poutible neutrino types, only $\nu_{c}$ are present in appreciable numbers due to the degenerecy of the electrons):

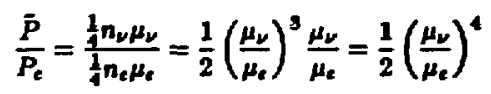

In the core, the reaction:

$$
n+\nu_{e} \Longleftrightarrow e+p
$$

gives the relation:

$$
\mu_{N}+\mu_{\nu}=\mu_{\varepsilon}+\mu_{P}
$$

Since $\rho$ is about nuclear density $\left(2.5 \times 10^{14} \mathrm{~g} / \mathrm{cc}\right), \bar{Z}=.33, \mu_{c}=11.1\left(\rho_{10} \bar{Z}\right)^{\frac{1}{2}} \mathrm{Mev}=230 \mathrm{Mev}$, and $\mu_{N} . p=30 \mathrm{Mev}$ (sec Bethe et al. (1979)), one finds $\mu_{\nu}=200 \mathrm{Mev}$. Using these values:

$$
\frac{\tilde{P}}{P_{e}}=.30
$$

Now the baryons can be tal: $\pi$ as a nonrelativistic degenerate fermion gas for purposes of the estimate being made here. So:

$$
P_{B}=\frac{1}{5} \frac{\hbar^{2}}{m_{B}}\left(3 \pi^{2}\right)^{\frac{2}{2}}\left(n_{N}^{\frac{4}{3}}+n_{P}^{\frac{5}{2}}\right)
$$

where $n_{N}$ is the number of neutrons/vol. and $n_{P}$ is the number of protons/vol. Using $P_{e}=\frac{1}{4} n_{e} \mu_{e}$, one finds: 


$$
\frac{\tilde{P}}{P}=\frac{\tilde{P}}{P_{c}+P_{B}}=\frac{\frac{p_{p}}{f_{c}}}{1+\frac{p_{g}}{P_{c}}}=.14
$$

The ratio $\tilde{E} / \mathrm{e}$ should be about the anme, and $00 \tilde{e}$ and $\bar{P}$ will be nellected acompared to $e$ and $P$ in the mass correction terms in (2). Therefore (2) can be rewritten a:

$$
\begin{gathered}
\left(1+\frac{e+P}{\rho c^{2}}\right) \cdot\left(\frac{1}{a} \frac{\partial v}{\partial t}+\frac{v}{a} \frac{\partial \ln \Gamma}{\partial t}\right) \\
=-\Gamma\left(\frac{\partial P}{\partial R}+\rho\left(1+\frac{e+P}{\rho c^{2}}\right) \frac{G M}{R^{2}}\left(1+\frac{4 \pi P R^{3}}{M c^{2}}\right) \frac{\left(1-\left(\frac{v}{c}\right)^{2}\right)}{\left(1-\frac{2 G M}{\partial c^{2}}\right)}\right) \\
-\Gamma \frac{\partial \tilde{P}}{\partial R}-\frac{1}{c^{2} b} \frac{\partial}{\partial t}\left(\frac{\tilde{G} b}{a}\right)-\frac{2 \Gamma H}{R}
\end{gathered}
$$

An estimate of the infall velocities can be made consistent with that quoted previously. When the supernova collapse begins, matter in the enyelope surrounding the dense white dwarf certral core of a red giant, previously in quasi-hydroatatic equilibrium, but auddenly without pr sssure support, begins co free fall. If it were to continue to free fall to the poxition of the original core surface, is would achieve velocities auch that:

$$
\stackrel{\because}{c} \approx \sqrt{\frac{2 G M}{R}} \approx .2
$$

where $M=1.4 M_{\odot}$ and $R=10^{7} \mathrm{~cm}$ are assumed. This is descriptive of pre-bounce conditions. If material could continue to free fall to typical neutron star radius, the above estimate would increase by a factor of 3 . The collapec evolution is not uimple free fall, however, as the rebounding shock wave produced when the core ceases to collapse, moves into the falling material, decelerating it. Also, in late times, the heating of matter behind the shock wave by neutrinos produces material pressure that further reduces the value of $v / c$ from the extreme estimate given above. As stated previously, peak values from the computer ouput indicate $v / c$ is usually less than .2 , and special relativistic effects are on the order of $4 \%$.

With the neglect of $(v / c)^{2}$ compared to unity, the right hand side of (3) can be written as:

$$
\text { R.H.S. }=-\Gamma\left(\frac{\partial P}{\partial R}+p\left(1+\frac{e+P}{\rho c^{2}}\right) \frac{G M}{R^{2}}\left(1+\frac{4 \pi P R^{3}}{M c^{2}}\right) \frac{1}{\left(1-\frac{2 G M}{R c^{2}}\right)}\right)+\text { neu. accel. }
$$


and:

$$
\Gamma=\left(1-\frac{2 G M}{R c^{3}}\right)^{\frac{1}{2}}
$$

$\Gamma$ can now be interpreted as giving the relation between increments in areal radius, and radius measured ferpendicular to a sphere centered on the star.

In the static limit, when there is no neutrino acceleration and no matter motion, (4) determines the structure of a neutron atar (ee Weinberg (1972)), and will give the exact structure (consistent with a given equation of state) of a central remnant, as the numerical evolution proceeds.

$\Gamma$ is unity at the center of the star, and reaches a minimum near the surface of the central remnant. Then is increases back to unity as $R$ increases outward. Using typical values of a neutron star mass and radius:

$$
\Gamma_{\min } \approx .8
$$

Time derivatives of $\Gamma$ during collapse are negligible as $\Gamma$ atays bracketed by $.8 \leq \Gamma \leq 1$, and so the terms in (3) involving of $a$ will be dropped.

From (A.43):

$$
M=4 \pi \int_{0}^{R}\left(\rho+\frac{e_{l o t}}{c^{2}}+\frac{\tilde{G} v}{c^{4}}\right) R^{2} d R \approx 1 \pi \int_{0}^{R}\left(\rho+\frac{e}{c^{2}}+\frac{\tilde{G} v}{c^{4}}\right) R^{2} d R
$$

An estimate of the size of $\tilde{G} v / \rho c^{4}$ can be made. This ratio should be largest just outside the neutrinosphere where the neutrinos begin to free stream. Now the mean free path of neutrinos can be estimated (see Lamb and Pethick (1976)):

$$
\frac{\lambda}{10^{6} \mathrm{~cm}}=\left(\frac{10^{12} \frac{\ell}{\rho c}}{\rho}\right)\left(\frac{10 M e v}{C_{\nu}}\right)^{2}
$$

So $\lambda$ is about the neutron star radius $\left(10^{6} \mathrm{~cm}\right)$ when $\rho \approx 10^{12} \mathrm{~g} / \mathrm{ce}$ and $\epsilon_{\nu} \approx 3 T_{\text {matter }} \approx$ $10 \mathrm{Mev}$.

Assuming the neutrino emission is blackbody, $\bar{G}=\frac{22}{8} \sigma_{B} T^{4}$ (note the $\frac{21}{8}$ comes from the fact that 6 neutrino types are present, and a fermion with one spin is worth $\frac{7}{16}$ of a photon, for blackbody radiation). Taking $v / c$ about .1 , and with the above values of $\rho$ and $T$, one finds: 


$$
\frac{\tilde{G} v}{\rho c^{4}} \approx 10^{-3}
$$

To a very good approximation:

$$
M=4 \pi \int_{0}^{R}\left(\rho+\frac{e}{c^{2}}\right) R^{2} d R
$$

The hydrodynamic equations now used in the supernova code are:

$$
\begin{gathered}
\left(1+\frac{e+P}{\rho c^{2}}\right) \rho\left(\frac{1}{a} \frac{\partial v}{\partial t}\right)=-\Gamma\left(\frac{\partial P}{\partial R}+\rho\left(1+\frac{e+P}{\rho c^{2}}\right) \frac{G M}{R^{2}}\left(1+\frac{4 \pi P R^{2}}{M c^{2}}\right) \frac{1}{\left(1-\frac{2 G M}{\partial c^{2}}\right)}\right) \\
-\Gamma\left(\frac{\partial \bar{P}}{\partial R}+\frac{1}{c^{2} b \Gamma} \frac{\partial}{\partial t}\left(\frac{\tilde{G} b}{a}\right)+\frac{2 H}{R}\right) \\
M=4 \pi \int_{0}^{R}\left(\rho+\frac{e}{c^{2}}\right) R^{2} d R \\
\frac{1}{a} \frac{\partial R}{\partial t}=\Gamma v
\end{gathered}
$$

Equations (5),(6),(7) differ from the equations in BW with the inclusion of the $a$ and $\Gamma$ factors, plus the first factor on the left hand aide of (5).

From Appendix A:

$$
a=\exp \int_{R}^{\infty} \frac{1}{c^{2}}\left(\left(\frac{\partial P_{t o t}}{\partial m}+\frac{2 H}{R} \frac{\partial R}{\partial m}+\frac{1}{c^{2}} \frac{\partial}{\partial t}\left(\frac{\bar{G} b}{a}\right)\right) \frac{d m}{\left(\rho+\frac{c \operatorname{cect} P_{e x}}{c^{2}}\right)}\right)
$$

or changing from an integral over $\mathrm{dm}$ to one over $\mathrm{dA}$, (A.38) becomes:

$$
a=\exp \int_{R}^{\infty} \frac{1}{c^{2}}\left(\left(\frac{\partial P_{\text {tot }}}{\partial R}+\frac{2 H}{R}+\frac{1}{c^{2} \Gamma b} \frac{\partial}{\partial t}\left(\frac{\tilde{G} b}{a}\right)\right) \frac{d R}{\left(\rho+\frac{\sec c+P_{\text {act }}}{c^{2}}\right)}\right)
$$

Making similar approximations in (8) as in (5) results in:

$$
a=\exp \int_{R}^{\infty} \frac{1}{c^{2}}\left(\left(\frac{\partial P_{\text {lot }}}{\partial R}+\frac{2 H}{R}+\frac{1}{c^{2} \Gamma b} \frac{\partial}{\partial t}\left(\frac{\tilde{G} b}{a}\right)\right) \frac{d R}{\left(\rho+\frac{e+P}{c^{2}}\right)}\right)
$$

In the static weak field limit:

$$
\frac{\partial P}{\partial R} \approx-\frac{\rho G M}{R^{2}}
$$


Assuming $R \geq \bar{R}$ where the mass outside $\bar{R}$ is negligible compared to the total mas of the system, then:

$$
a \approx \exp \int_{R}^{\infty} \frac{1}{c^{2}}\left(-\frac{G M}{R^{2}}\right) d R=\exp \left(\frac{-G M}{R c^{2}}\right)=e^{-\frac{4}{2}} \approx 1+\frac{\phi}{c^{2}}
$$

where $\frac{\phi}{c^{2}} \ll 1$, and $\phi$ is the Newtonian gravitational potential. (Notice that in this case $\Gamma \simeq a$, i.e. the Schwarzschild metric.)

Thus it is seen that $a$ represents a generalization of the gravitational alowing down of clocks to the dynamic case. In the supernova code, a is normalized to unity at the outer boundary of the star, in this way, the time measured on the outer boundary ia proper time. The equation used in the supernova code for a is:

$$
a=\exp \int_{R}^{R_{\text {mas }}} \frac{1}{c^{2}} \frac{\partial P}{\partial R} \frac{d R}{\left(\rho+\frac{\varepsilon+P}{c^{2}}\right)}
$$

In (10), the neutrino acceleration terms have been neglected since they are small compared to the matter pressure acceleration (see the previous estimate of the ratio $\tilde{P} / P$ in the central part of the star).

MASS CONSERVATION

Equation (A.45) is rewritten below:

$$
\frac{1}{a} \frac{\partial}{\partial t} \ln \left(\rho R^{2}\right)+\frac{\partial U}{\partial m} / \frac{\partial R}{\partial m}=0
$$

using:

$$
\begin{gathered}
\frac{\partial U}{\partial m} / \frac{\partial R}{\partial m}=\frac{\partial U}{\partial R} \\
U \approx \Gamma v
\end{gathered}
$$

Equation (A.45) becomes:

$$
\frac{1}{a} \frac{\partial \rho}{\partial t}+\frac{\rho}{R^{2}} \frac{\partial}{\partial R}\left(R^{2} \Gamma v\right)=0
$$

This differs from the BW equation with the retention of the factors of $a$ and $\Gamma$.

\section{ENERGY CONSERVATION}

From Appendix A: 


$$
\frac{1}{a} \frac{\partial(e / \rho)}{\partial t}+\frac{P}{a} \frac{\partial}{\partial t}\left(\frac{1}{\rho}\right)=-\int c k(B-F) d \mu \frac{d Q}{a}
$$

This can be rewritte, 1 as:

$$
\frac{1}{a} \frac{\partial(e / \rho)}{\partial t}+\frac{P}{a} \frac{\partial}{\partial t}\left(\frac{1}{\rho}\right)=\frac{T}{a} \frac{\partial S}{\partial t}
$$

with:

$$
\frac{T}{a} \frac{\partial S}{\partial t}=-\int c k(B-F) d \mu \frac{d Q}{a}
$$

The right hand side of (12) is identified with $T \frac{g s}{\partial t}$, giving the change in entropy for the matter in terms of the neutrinos. The left band side of (12) is used as it atands in the supernova code (again the $\frac{1}{2}$ factors are additions to the code deacribed in $\mathrm{BW}$ ). The opacities are discussed later in Appendix B.

In a study of antineutrino production during gravitational collapse (eee Wooley, Wilson, and Mayle (1985)), a $500000 M_{\odot}$ atar was followed both with the aupernove code using $(5),(6),(7),(10),(11),(12)$, and a fully general relativiatic collapue code. (This code is a 1-dimensional version of a 2-dimensional collapse code used to study the production of gravitational waves. It was kindly provided by Charles Evans (1984).) This large star has a high entropy going into collapse ( entropy/baryon $\simeq 200 k_{B}$ ), and the neutrino production had a negligible effect on the dynamies of collapse, so an adiabatic collapse (used in the fully general relativistic code) is a good approximation. Both codes showed the trapped surface forming at the same interior mass, indicating that at least in this case, the approximation in the supernova code is very good.

\section{NEUTRINO TRANSPORT}

Equation (A.46) is rewritten below:

$$
\begin{gathered}
\frac{1}{c a} \frac{\partial F}{\partial t}=-\frac{\mu \Gamma}{a R^{2}} \frac{\partial}{\partial R}\left(a R^{2} F\right)-\Gamma\left(\frac{1}{R}-\frac{\partial}{\partial R} \ln a\right)\left(\frac{\partial}{\partial \mu}\left(F\left(1-\mu^{2}\right)\right)\right) \\
+\frac{F}{c a \rho} \frac{\partial \rho}{\partial t}+\frac{R}{c} \frac{\partial}{\partial R}\left(\frac{U}{R}\right)\left(\frac{\partial}{\partial \mu}\left(\mu\left(1-\mu^{2}\right) F\right)+\mu^{2} Q \frac{\partial F}{\partial Q}\right) \\
+\frac{Q}{c a} \frac{\partial F}{\partial Q}\left(\frac{\partial}{\partial t} \ln \frac{R}{a}\right)+k \rho(B-F)
\end{gathered}
$$

Now defining: 


$$
\begin{aligned}
& E_{q}=\int_{-1}^{2} F d \mu \\
& G_{q}=\int_{-1}^{1} C F \mu d \mu \\
& F_{q}=\int_{-1}^{1} F \mu^{2} d \mu
\end{aligned}
$$

and integrating (A.46) over $\mathrm{d} \mu$ results in:

$$
\begin{aligned}
& \frac{1}{a} \frac{\partial E_{q}}{\partial t}=-\frac{\Gamma}{a R^{2}} \frac{\partial}{\partial R}\left(a R^{2} G_{q}\right)-Q \frac{\partial E_{q}}{\partial Q} \frac{\partial \ln a}{a \partial t}+\frac{E_{q}}{a \rho} \frac{\partial \rho}{\partial t} \\
& +R \frac{\partial}{\partial R}\left(\frac{U}{R}\right) Q \frac{\partial P_{q}}{\partial Q}+Q \frac{\partial E_{q}}{\partial Q} \frac{U}{R}+\int_{-1}^{1} \operatorname{ck\rho }(B-F) d \mu
\end{aligned}
$$

Now replace in the above:

$$
R \frac{\partial}{\partial R}\left(\frac{U}{R}\right) Q \frac{\partial \bar{P}}{\partial Q}+Q \frac{\partial \bar{E}_{G}}{\partial Q} \frac{U}{R}
$$

with:

$$
Q \frac{\partial}{\partial Q}\left(\frac{\bar{E}_{q}}{R^{2}} \frac{\partial}{\partial R}\left(\frac{D}{\lambda c} r^{2} U\right)\right)
$$

where $\mathrm{D}$ is the diffusion coefficient (see following discussion).

This is part of an approximation called flux limited diffusion. It is a means (somewhat ad hoc, but see Levermore (1979)) to close the moment equations in such a way that in the diffusion limit, $\frac{D}{\lambda c}=\frac{1}{3}$ (equivalent to $E_{q}=3 P_{q}$, in which the replacement above is an exact equality, $\lambda$ is the geutrino mean free path). In the free streaming limit:

$$
\frac{D}{\lambda c}=\frac{1}{\lambda\left|\frac{\partial \ln E_{q}}{\partial R}\right|}
$$

The free streaming limit is dictated by the following identification:

$$
\bar{G}_{q}=-D \frac{\partial E_{q}}{\partial R}
$$

using:

$$
D=\frac{c}{\left|\frac{\partial \ln E_{q}}{\partial R}\right|}=-\frac{c \bar{E}_{q}}{\frac{\partial E_{q}}{\partial R}}
$$

then: 


$$
c_{q}=\frac{c E_{q}}{\frac{\partial E_{q}}{\partial R}} \frac{\partial E_{q}}{\partial R}=c E_{q}
$$

This is the exact result for free atreaming. In the diffusion limit $D=\frac{1}{3} \lambda e$ so:

$$
\zeta_{q}=-\frac{1}{3} \lambda c \frac{\partial E_{q}}{\partial R}
$$

The form chosen for $\mathrm{D}$ is arbitrary, but must have the appropiate aymptotic behavior. One simple choice is:

$$
D=\frac{\lambda c}{3+\lambda\left|\frac{\partial \ln E_{1}}{\partial f}\right| \xi}
$$

with:

$$
\begin{gathered}
\xi=1+\frac{3}{1+\frac{z}{2}+\frac{z^{2}}{\delta}} \\
x=\lambda\left|\frac{\partial \ln E_{q}}{\partial R}\right|
\end{gathered}
$$

More is said about the validity of this approximation in Chapter IV.

With the flux limited diffusion approximation, (13) becomes:

$$
\begin{gathered}
\frac{1}{a} \frac{\partial E_{q}}{\partial t}=-\frac{\Gamma}{a R^{2}} \frac{\partial}{\partial R}\left(a R^{2} \bar{G}_{q}\right)-Q \frac{\partial E_{q}}{\partial Q} \frac{\partial \ln a}{a \partial t}+\frac{E_{q}}{a \rho} \frac{\partial \rho}{\partial t} \\
+Q \frac{\partial}{\partial Q}\left(\frac{\bar{E}_{q}}{R^{2}} \frac{\partial}{\partial R}\left(\frac{D}{\lambda c} R^{2} \Gamma v\right)\right)+\int_{-1}^{1} c k \rho(B-F) d \mu
\end{gathered}
$$

Since:

$$
\begin{aligned}
& \tilde{e}=\int_{0}^{\infty} \bar{E}_{q} \frac{d Q}{a} \\
& \tilde{G}=\int_{0}^{\infty} \bar{G}_{q} \frac{d Q}{a} \\
& \tilde{P}=\int_{0}^{\infty} P_{q} \frac{d Q}{a}
\end{aligned}
$$

integration of both sides of (16) over $d Q$ and dividing both sides by $a$, one finds:

$$
\begin{gathered}
\frac{1}{a} \frac{\partial \tilde{e}}{\partial t}=-\frac{\Gamma}{a^{2}} \frac{1}{R^{2}} \frac{\partial}{\partial R}\left(a^{2} R^{2} \tilde{G}\right)+\frac{\tilde{e}}{a \rho} \frac{\partial \rho}{\partial t}-\int\left(\frac{E}{R^{2}} \frac{\partial}{\partial R}\left(\frac{R^{2} D \Gamma v}{\lambda c}\right)\right) d Q \\
+\int_{0}^{\infty} \int_{-1}^{1} \operatorname{ck\rho }(B-F) d \mu \frac{d Q}{a}
\end{gathered}
$$


Note that in the static free atreaming limit one finds from (17):

$$
\tilde{G}=\frac{\text { const }}{a^{2} R^{2}}
$$

The $a^{2}$ factor in the denominator of (18) is the expected redshift for the energy luminosity of free streaming neutrinos.

Now, the flux limited diffusion approximation determines the neutrino acceleration of the matter if the demand of energy and momentum conservation is required. First, I will write the expression for matter acceleration, then show that it in the correct one to be consistent with fux limited diffusion. Equation (5) becomes:

$$
\begin{aligned}
\left(1+\frac{e+P}{\rho c^{2}}\right) \frac{\rho}{a} \frac{\partial v}{\partial t}= & -\Gamma\left(\frac{\partial P}{\partial R}+\rho\left(1+\frac{e+P}{\rho c^{2}}\right) \frac{G M}{R^{2}}\left(1+\frac{4 \pi R^{3} P}{M c^{2}}\right) \frac{1}{\left(1-\frac{2 G M}{R c^{2}}\right)}\right) \\
& +\Gamma\left(\int_{0}^{\infty} \frac{\bar{G}_{q}}{\lambda c} \frac{d Q}{a}+\frac{\partial \ln a}{\partial R} \int_{0}^{\infty} \frac{D}{\lambda c} E_{q} \frac{d Q}{a}\right)
\end{aligned}
$$

An equation for total energy density (i.e. $\frac{1}{2} \rho v^{2}+e+\tilde{\varepsilon}$, with the form of the kinetic energy in the Newtonian limit) should be constructed using (11),(12), (17), and (19). However, since the general relativistic mass correction term on the left hand side of (19) is only important in the very center of the star, where $v / c \leq .001$ and neutrinos are trapped, for purposes of this derivation oniy, (19) will be replaced by equation (20) below:

$$
\frac{\rho}{a} \frac{\partial v}{\partial t}=-\Gamma\left(\frac{\partial P}{\partial R}+F_{\mathrm{grav}}-\int_{0}^{\infty} \frac{\bar{G}_{q}}{\lambda c} \frac{d Q}{a}-\frac{\partial \ln a}{\partial R} \int_{0}^{\infty} \frac{D}{\lambda c} E_{q} \frac{d Q}{a}\right)
$$

with:

$$
F_{\text {grav }}=\rho\left(1+\frac{e+P}{\rho c^{2}}\right) \frac{G M}{K^{3}}\left(1+\frac{4 \pi R^{3} P}{M c^{2}}\right) \frac{1}{\left(1-\frac{3 G M}{R c^{2}}\right)}
$$

The right hand sides of (19) and (20) are identical.

Now:

$$
\begin{gathered}
\frac{1}{a} \frac{\partial}{\partial t}\left(\frac{1}{2} \rho v^{2}\right)=\frac{v^{2}}{2 a} \frac{\partial \rho}{\partial t}+\frac{\rho v}{a} \frac{\partial v}{\partial t} \\
=-\frac{v^{2} \rho}{2} \frac{1}{R^{2}} \frac{\partial}{\partial R}\left(R^{2} \Gamma v\right)-v \Gamma \frac{\partial P}{\partial R}+v \Gamma \int_{0}^{\infty} \frac{\bar{G}_{q}}{\lambda c} \frac{d Q}{a} \\
+v \Gamma \frac{\partial \ln a}{\partial R} \int_{0}^{\infty} \frac{D}{\lambda c} \bar{E}_{q} \frac{d Q}{a}-\Gamma v F_{\text {grav }}
\end{gathered}
$$

Using (12),(17), and (21), one finds (where the time derivative is now locally Eulerian, not Lagrangian): 


$$
\begin{aligned}
\frac{\partial}{\partial \tau}\left(\frac{1}{2} \rho v^{2}+e+\bar{\epsilon}\right)+\frac{1}{R^{2}} \frac{\partial}{\partial R} & \left(\Gamma R ^ { 2 } \left(v\left(\frac{1}{2} \rho v^{2}+e+\tilde{c}+P+\int \frac{\left.\left.\left.D E_{Q} \frac{d Q}{\lambda c}\right)+\bar{G}\right)\right)}{a}\right)\right.\right. \\
= & -\Gamma v F_{\text {res }}-\bar{G} \mathrm{r} \frac{\partial \ln \frac{e^{2}}{\partial R}}{\partial R}
\end{aligned}
$$

$=$ work done by gravity on matter and nevtrinos

From (22) it is thus seen that che approximation to general relativity used is not Post Newtonian, but one in which the static effects of general relativity are done correctly (i.e. gravitational force, redshift of neutrinos), but the matter kinetic terms are treated in the Newtonian approximation.

Th.e above discussion assumed only one type of neutrino. However, six different typss of neutrinos are present, the electron neutrino, mu neutrino, tau neutriro, and their axtiparticles. The transport equation (17) will apply to each type, with different source and sink terms for the possible interactions with matter. There will be a consribution to (12), the matter internal energy equation, and (19), the ratter acceleration for each type of neutrino.

A brief discussion of opacity, and some differences between the opacities used in BW, and what is presently used in the supernova code will be found in Appendix B.

\section{LEPTON NUMBER CONSERVATION}

Finally, equation (A.48) is repeated below:

$$
\frac{\rho}{m_{P}} \frac{1}{a} \frac{\partial \bar{Z}}{\partial t}=-\int_{0}^{\infty} \int_{-1}^{1}(B-F) \frac{d Q}{Q} d \mu
$$

This equation is now used in the supernova code, with contributions from the electron neutrino and antineutrino on the right hand side.

\section{SUMMARY}

In this chapter the new equations (the old equations are found in Bowers and Wilson $(1982 \mathrm{a}, \mathrm{b})$ ) used to model a supernova are presented. The new physics that is being auded ss the gravitational redshift of the neutrinos produced in the collapse. Estimates are made of the importance of its inclusion to the late time supernova explosion. (Other improvernents in the physics now used in the supernova code are presented in Appendix B.) 


\section{Chapter III References}

Baron, E., Cooperstein, J. and Kahana, S. 1985, Phys. Rev. Lettr., v55., 126.

Bethe, H.A., Brown, G.E., Applegate J. and Lattimer, J. 1979, Nucl. Phys., A324, 487.

Bowers, R.L., and Wilson, J.R. 1982a, Ap. J. Suppl., v50, 115.

-. 1982b, Ap. J., v263, 366.

Evans, C. 1984 PhD Thesis, University of Texas at Austin

Lamb, D.Q. and Pethick, C.J. 1976, Ap. J. Lettr., v209, 177.

Levermore, C.D. 1979, Lawrence Livermore Report UCID-18229

May, M., and White, R.H. 1967 , Meth. Comp. Phys., v73, 219.

Weinberg, S. 1972, "Gravitation and Cosmology" (John Wiley and Sons, Inc.: New York), Chap.11

Wilson, J.R. 1971, Ap. J., v163, 209.

Woosley, S.E., Wilson, J.R., Mayle, R. 1985, Ap. J., accepted for publication. 


\section{Chapter IV}

\section{NEUTRINO TRANSPORT}

Is Chapter III, the flux limited diffusion approximation for neutrino transport was introduced. This chapter is concerned with the validity of replacing the Boltzmann equation with the flux limited diffusion epproximation.

\section{Neutrino Transport(Flux Limited Differion Approximation)}

The supernova computer code transports electron neutrinos using multigroup fiux limited diffusion. The following equation determines the neutrino evolution:

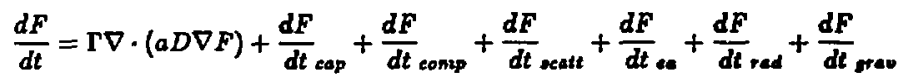

where the energy density of electron neutrinos at radius $R$ is $\int F \frac{Q Q}{a}$ and $D$ is the diffusion coefficient. D contains a flux limiter that forces $F\left(\frac{Q}{a}, R\right)$ to be proportional to $\frac{1}{k^{2}}$ in the regions where neutrino3 are free streaming. $Q$ is the energy of a neutrino as measured by an observer at infinity, while $\frac{Q}{a}$ is the locally measured energy (see Chapter III). The form used for $\mathrm{D}$ is as follows:

$$
D=\frac{\lambda c}{3+\lambda\left|\frac{d n F}{d \xi}\right| \xi}
$$

where:

$$
\xi=1+\frac{3}{1+\frac{z^{2}}{2}+\frac{t^{2}}{8}}
$$

and:

$$
x=\lambda\left|\frac{d \ln F}{d R}\right|
$$

This particular form for $D$ was arrived at by Wilson (private communication) when photon radiation transport was being studied.

The terms on the right hand side of (1) include changes in the neutrino field due diffusion, electron capture, changes in volume, scattering, emission and absorbtion, and work done on the matter. The last term in (1), as well as the factors of $a$ and $r$, are due to goneral relativity (see Chapter IlI).

Looking at equation (16) in Chapter III, one can make the following identifications with (1) of this chapter: 


$$
\begin{aligned}
& \frac{d F}{d t} \text { ces }+\frac{d F}{d t} \text { sealt }+\frac{d F}{d t} \text { as } \equiv a \int_{-2}^{1} c k p(B-F) d \mu \\
& \frac{d F}{d t}_{\text {red }}=a Q \frac{\partial}{\partial Q}\left(\frac{F}{R^{2}} \frac{\partial}{\partial R}\left(\frac{D}{\lambda c} R^{2} \Gamma_{0}\right)\right. \\
& \frac{d F}{d t \text { comp }}=\frac{F}{\rho} \frac{\partial \rho}{\partial t}
\end{aligned}
$$

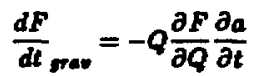

and the same symbol, $F$, has been uned for $F\left(\frac{Q}{4}, R, t, \mu\right)$ and $F\left(\frac{Q}{a}, R, t\right)=\int F\left(\frac{Q}{a}, R, t, \mu\right) d \mu$.

The electı na antineutrinos and mu-tau neutrino and antineutrinon are vimilarly treated except that the capture term in (1) is not included.

\section{Boltzmann Transport Equation}

Let $f$ represent the distribution function of electron neutrinos such that:

$$
\int f(\vec{x}, \vec{p}, t) \frac{d^{3} p}{h^{3}}=\text { number density of neutrinos }
$$

In the limit of complete thermodynamic equilibrium:

$$
f(\vec{x}, \vec{p}, \lambda)=\frac{1}{\exp \left(\frac{E}{k_{B} \vec{T}}\right)+1}
$$

The transport equation for $f(\vec{x}, \vec{p}, t)$ is taken to be:

$$
\begin{aligned}
\frac{1}{c} \frac{\partial f}{\partial t}+\hat{p} \cdot \nabla f= & k_{\mathrm{a}} \rho(b-f)-f \int\left(1-f^{\prime}\right) \rho \kappa_{,}\left(E, \Omega \rightarrow E^{\prime}, \Omega^{\prime}\right) \frac{d^{3} p^{\prime}}{h^{3}} \\
& +(1-f) \int f^{\prime} \rho \kappa_{a}\left(E^{\prime}, \Omega^{\prime} \rightarrow E, \Omega\right) \frac{d^{3} p^{\prime}}{h^{3}}
\end{aligned}
$$

On the right har $d$ side of (2), the first term represents absorbtion and emiesion of neutrinos by free nucleons, and $b$ is a Fermi-Dirac distribution function representing emission with $\mu_{\nu}=\epsilon_{N}-\epsilon_{p}+\mu_{e}$. The sr:ond and third terms represent scattering by free nucleons. electrons an? positrons, and helium nuclei.

Since the accuracy of the neutrino transport is most important to the late time supernova explosions at late times, the comparison with the flux limited diffusion approximation is A.w. siter bounce, ut which time only the above sources of opecity are considered important (i.e. the heavy nuclei are all dissociated). This occurs in the region of the star from the center to the shock position. 
Equation (2) neglects Doppler shift, Doppler aberration effects, adrection, and gravitational redshift. After bounce, the core is quaci-static and all the above effecta are amall except the gravitational redshift (ree Chapter ID). The gravitational redabift in taken into ccount after the final spectrum is nolved for, by using the energy shift that geutrinos would undergo if emitted from the neutrinosphere. In (2), the term of will be neglected, and the equation solved in the static approximation.

The construction of the opacities for use in (2) is discumed next. First, an eatimate of the importance of electron-powitron acattering opacity as compared to the total opacity is made. Helium will be ignored as an opacity source for purposes of making this eatimate.

The ratio of electron-positron scattering to total opacity is written a:

$$
\frac{k_{e}^{e}}{k_{\text {tot }}}=\frac{n_{e}-\sigma_{e-\nu}+n_{e}+\sigma_{e+\nu}}{n_{N} \sigma_{N \nu}{ }^{a}+n_{N} \sigma_{N \nu}^{\prime}+n_{P} \sigma_{P_{\nu}}^{\prime}+n_{e}-\sigma_{e-\nu}+n_{e}+\sigma_{e}+\nu}
$$

where:

$$
\begin{gathered}
\sigma_{N \nu}^{a} \approx\left(\frac{1+3 \alpha^{2}}{4}\right) \sigma_{o}\left(\frac{E}{m_{e} c^{2}}\right)^{2} \\
\sigma_{N \nu}^{a} \approx \sigma_{0}\left(1-C_{A}\right)^{2}\left(\frac{E}{m_{e} c^{2}}\right)^{2} \\
\sigma_{P \nu}^{f} \approx \frac{\sigma_{O}}{4}\left(\left(C_{V}-1\right)^{2}+3 \alpha^{2}\left(C_{A}-1\right)^{2}\right)\left(\frac{E}{m_{e} c^{2}}\right)^{2}
\end{gathered}
$$

and $\sigma_{0}=1.7 \times 10^{-14} \mathrm{~cm}^{2}, E$ is the neutrino energy. $C_{V}$ and $C_{\mathcal{A}}$ are weak interaction constants and $\alpha \approx 1.2$ is a strong interaction constant. The cross-sections are zaken from Tubbs and Schramm (1975), bereafter denoted by TS.

If $C_{V} \approx 1, C_{A}=.5$, then $\sigma_{P_{\nu}}^{\prime} \approx \sigma_{N_{\nu}}^{\prime}$ (since $\alpha \approx 1.2$ ). For the number of pairs per unit volume the following holds:

$$
n_{p a i r d}=n_{e}+=\int \frac{1}{\exp \left(\frac{E+\mu_{e}}{k_{B} T}\right)+1} \frac{d^{3} p}{h^{3}} \approx \frac{1}{\pi^{3}}\left(\frac{m_{\varepsilon} c}{\hbar}\right)^{3}\left(\frac{k_{B} T}{m_{e} c^{2}}\right)^{3} F_{z}^{-}(\eta)
$$

where the final expression is good in the limit $\frac{m_{r} e^{2}}{k_{B} T} \ll 1$. The symbol $F_{2}^{-}(\eta)$ is defined as:

$$
F_{2}^{-}(\eta)=\int_{0}^{\infty} \frac{x^{2} d x}{e^{x+\eta}+1}
$$

with $\eta=\frac{\mu_{c}}{k_{D} T} \cdot F_{2}^{-}(\eta) \approx 2 e^{-\eta}$ is an approximation good to within $10 \%$.

In the limit of degenerate electrons with $C_{V}=1$ and $C_{A}=.5$ : 


$$
\sigma_{\varepsilon-\nu}=\frac{T}{32} \sigma_{*} \frac{E \mu_{c}}{\left(m_{\varepsilon} c^{2}\right)^{2}}
$$

This cron-section in taken from TS.

If the electrons are degenerate, the number of positrons in negligible. In this limit:

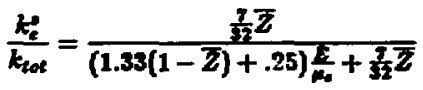

In this case $\left\langle E>_{\infty} \mu_{e}\right.$, and taking $\bar{Z}$, the number of matter electrons per baryon, to be about $\frac{1}{3}$, one finds:

$$
\frac{k_{e}^{\prime}}{k_{\text {lot }}}=.06
$$

Therefore electron seattering is completely negligible in the degenerate limit.

If electrons and positrons are relativistic and non-degenerate then (taking $C_{V}=1$ and $\left.C_{A}=.5\right):$

$$
\begin{aligned}
& \sigma_{e-\nu}=\frac{7}{8} \sigma_{0} \frac{E k_{B} T}{\left(m_{e} c^{2}\right)^{2}} \\
& \sigma_{e+\nu}=\frac{3}{8} \sigma_{o} \frac{E k_{B} T}{\left(m_{e} c^{2}\right)^{2}}
\end{aligned}
$$

where the cross-sections are taken from TS.

Therefore in the limit of relativistic non-degenerate electrons and poeitrons:

$$
\frac{k_{e}^{0}}{k_{t o l}}=\frac{\frac{5.5}{n z} T^{3} e^{-\eta}+\frac{7}{8} \bar{Z}}{(1.33(1-\bar{Z})+.25) \frac{E}{2}+\frac{5.5}{17} T^{3} e^{-\eta}+\frac{7}{8} \bar{Z}}
$$

where $\rho_{\tau}=\rho \times 10^{-7}$ with $\rho$ in $\frac{\ell}{\epsilon \in}, T$ and $E$ are in Mev, and the approximation for $F_{2}^{-}(\eta)$ mentioned above has been used.

There is another effect that should be accounted for in the above. The scattering opacities should be transport opacities (i.e. opacities weighted by the factor $1-\cos \theta$, where $\theta$ is the scattering angle). Using data taken from TS, a fit for $\cos \theta \operatorname{can}$ be made, and (3) can be changed $t o$ include this factor. The fit for $\cos \theta$ used is:

$$
\cos \theta=\frac{E}{4 k_{B} T+\mu_{\varepsilon}+E}
$$

It can be seen that is $E \gg 4 k_{B} T+\mu_{e}$, then the electron positron scattering will also not be important. 
To estimate the value of (3) use the fact that $\bar{Z} \leq \frac{1}{3}$ in the region of interent, and that the electrons are slightly degenerate $\left(\eta \approx \frac{1}{j}\right)$ to find:

$$
\frac{k_{i}^{3}}{k_{\text {tec }}}=\frac{\frac{1.8}{n} T^{3}+.29}{3.4+\frac{1.5}{n} T^{3}+.29}
$$

where the moat restrictive value of $\bar{Z}$ (i.e. 1) has been taken. Demanding that this be less than .20 results in the condition $p 7 \geq 8 T^{3}$, with $T$ in Mer. Looking at a computer simulation, this relotion is generally seen to hold during the late time evolution in the region from the center to near the shock position.

Since nucleons dominate the opacity, the electron-positron scattering contribution may be approximated without greatly effecting the calculated spectrum.

From the above estimate for electron-positron acattering opucity, it is ween that:

$$
k_{\varepsilon}^{*}\left(\mu_{\varepsilon}, T, E\right)=E H\left(\mu_{\varepsilon}, T, E\right)
$$

where $H\left(\mu_{e}, T, E\right)$ is a more slowly varying function of $E$ than $k_{e}\left(\mu_{e}, T, E\right)$. Figure 1 a and 1b show graphs of $\frac{\sigma_{u f}}{E T}$ versus $\log \left(\frac{k_{g} T}{\mu_{1}}\right)$ constructed using the results of TS. In the high temperature limit $H\left(\mu_{e}, T, E\right)$ becomes independent of $E$, as can be aeen from figures 1 a and $1 b$.

Now write:

$$
f \int\left(1-f^{\prime}\right) \kappa_{e}^{\prime}\left(E, \mathrm{n} \rightarrow E^{\prime}, \Omega^{\prime}\right) \frac{d^{3} p^{\prime}}{h^{3}}=k_{\varepsilon}^{\prime}<1-f^{\prime}>f
$$

where $\left\langle 1-f^{\prime}\right\rangle$ is some average blocking factor.

The other term involving electron-positron acattering will be approximated in such a way that, in the limit $f=e^{\frac{-E}{E_{B}}}$, the electron-positron scattering terms will cancel (with the neglect of the blocking factors). This condition results in:

$$
(1-f) \int f^{\prime} \kappa_{e}^{\prime}\left(E^{\prime}, \Omega^{\prime} \rightarrow E, \Omega\right) \frac{d^{3} p^{\prime}}{h^{3}}=\frac{E}{4 \pi k_{B} T}(1-f) \int_{E}^{\infty} \int_{1 \pi} k_{e}^{a}\left(\mu_{e}, T, E^{\prime}\right) \frac{f^{\prime}}{E^{\prime}} d E^{\prime} d \Omega^{\prime}
$$

To show this is correct, the integrals on the right hand side of the above equation need to be done with $f=e^{\frac{-E}{k^{T}}}$. To do the energy integral, take $H\left(\mu_{e}, T, E\right)$ outside the integral so that:

$$
\frac{E}{4 \pi k_{B} T} \int_{E}^{\infty} \int_{4 \pi} k_{e}^{\prime}\left(\mu_{e}, T, E^{\prime}\right) e^{\frac{\mu-E^{\prime}}{k_{B} T}} \frac{d E^{\prime}}{E^{\prime}} d \Omega^{\prime}=\frac{H\left(\mu_{e}, T, E\right) E}{k_{B} T} \int_{E}^{\infty} e^{\frac{-E^{\prime}}{k_{B} T}} d E^{\prime}=k_{e}^{\prime} f
$$


This is a very good approximation in the limit $f_{f}<1$, aince in this case, $k \propto E$ and $H\left(\mu_{e}, T, E\right)$ is independent of $E$. This is aleo the region where $f=e^{-5}$, if the neutrinos are in equilibrium.

The nucleon scattering terms are simpler in form since the seattering is conservative. The proton differential crossection has an angular dependence such that:

$$
\frac{d \sigma}{d \Omega} \propto\left(1-\frac{1}{3} \cos \theta\right)
$$

and the neutron crossection is isotropic (sc: TS). The nucleon scattering terms will be written as:

$$
-(1-f) k_{0}^{N+P} f+(1-f) k_{0}^{N+P} \frac{1}{4 \pi} \int_{4 \pi} f^{\prime} \mathrm{d}^{\prime}
$$

where the angular dependence of the croas-section bas been averaged over $4 \pi$, weighted by $(1-\cos \theta)$ in order to produce a transport crose section. Notice that this is another approximation as the angular dependence of the cross-section really should appear inside the angular integral over $f$.

The scattering cross-section for belium is taken from TS and is given by the following expression:

$$
\sigma_{H_{e}}=\frac{8}{3} \sigma_{\circ}\left(\frac{E}{m_{a} c^{2}}\right)^{2} \sin ^{2} \theta_{W}
$$

where $\theta_{W}$ is the Weinberg angle. The above has been averaged over $4 \pi$ steradians weighted by $(1-\cos \theta)$.

Since both helium and the neutron-proton scattering opacity have the same dependence on the neutrino energy, the scattering opacity for these particles may be added to find a total conservative scattering opacity for use in the transport equation.

The Boltzmann equation is rewritten below:

$$
\begin{gathered}
\hat{p} \cdot \nabla f=\rho k_{\mathrm{a}}^{N+P}(b-f)-\rho k_{e}^{*}<1-f^{\prime}>f+\frac{E(1-f)}{4 \pi k_{B} T} \int_{E}^{\infty} \int_{\Delta \pi} \rho k_{e}^{*} \frac{f^{\prime}}{E^{\prime}} d E^{\prime} d \Omega^{\prime} \\
-(1-f) \rho k_{a}^{C} f+(1-f) \rho k_{*}^{C} \frac{1}{4 \pi} \int_{6 \pi} f^{\prime} d \Omega^{\prime}
\end{gathered}
$$

where:

$$
k_{e}^{C}=k_{a}^{N+P}+k_{p}^{H e}
$$

Next, introduce a new distribution function $F(\vec{x}, \hat{p}, E)$ such that: 


$$
F(\vec{x}, \hat{p}, E)=\left(\frac{E}{h c}\right)^{3} f(\vec{p}, \vec{x})
$$

This is done since the supernova code uses the angular average of $F(\vec{x}, \hat{P}, E)$ a the neutrino distribution function (i.e. $\left.F_{\text {eode }}=\int \Gamma(\bar{x}, \hat{p}, E) a n\right)$ ).

The transport equation for $\boldsymbol{F}(\vec{x}, \hat{p}, E)$ becomea:

$$
\begin{aligned}
& \hat{p} \cdot \nabla F=\rho k_{a}^{N+P}(B-F)-\rho k_{e}^{*}<1-f^{\prime}>F+\frac{E^{4}}{4 \pi k_{B} T}(1-f) \int_{E}^{\infty} \int_{a r} \rho k_{e}^{*} \frac{F^{\prime}}{E^{\prime}} d E^{\prime} d n^{\prime} \\
& -(1-f) \rho k_{i}^{C} F+(1-f) \rho k_{i}^{c} \frac{1}{4 \pi} \int_{4 \pi} F^{\prime} d R^{\prime}
\end{aligned}
$$

where $B=\frac{E^{3}}{(h e)^{3} b \text {. }}$

In order to solve the Boltzmann transport equation, the denaity, temperature, and composition profile must be provided so that the numerical value of the opacity can be found. One approximation is to use the density, temperature, and composition profile from the output of a computer simulation generated using the flux limited difiusion approximation. This should be an acceptable proceedure, to the extent that the two methods of neutrino transport give similar results.

The blocking factors $\left\langle 1-f^{\prime}\right\rangle$ and $(1-f)$ are also evaluated using the value of $f$ obtained from the supernova calculation. The supernove code uned 16 energy bins and the following are taken for the blocking factors:

$$
\left.<1-f^{\prime}\right\rangle=\frac{\sum_{\text {hins }}\left(1-\frac{F\left(E^{\prime}, \bar{x}\right)}{1 \pi E^{3}}(h c)^{3}\right) E^{\prime 2} \Delta E_{\text {bin }}^{\prime}}{\sum_{\text {bins }} E^{\prime 2} \Delta E_{\text {bin }}^{\prime}}
$$

and:

$$
1-f=1-\frac{F(E, \vec{x})}{4 \pi E^{3}}(h c)^{3}
$$

One collapse problem, a $15 M_{\odot}$ star, was run with 32 energy bins and weak sensitivity to bin number was found in the calculated spectrum.

Equation (4) is still difficult to solve since it is a differential-integral equation for $F(\vec{x}, \hat{p}, E)$. In the spirit of the proceeding approximations, the spectrum produced by the supernova simulation is used to evaluate the integral involving the electron-positron scattering since, as was shown earlier, it is much less important than the nuclear contribution. Equation (4) can be rewritten as: 


$$
F(\vec{x}, \hat{p}, E)=\left(\frac{E}{h c}\right)^{3} f(\vec{p}, \vec{x})
$$

This is done since the supernova code uses the angular average of $F(\vec{x}, \hat{p}, E)$ a the neutrino distribution function (i.e. $\left.F_{\text {eode }}=\int F(\bar{x}, \hat{p}, E) d n\right)$ ).

The transport equation for $F(\vec{x}, \hat{p}, E)$ becomes:

$$
\begin{aligned}
& \hat{p} \cdot \nabla F=\rho k_{a}^{N+P}(B-F)-\rho k_{\varepsilon}^{0}<1-f^{\prime}>F+\frac{E^{4}}{4 \pi k_{B} T}(1-f) \int_{E}^{\infty} \int_{4 r} \rho k_{e}^{*} \frac{F^{\prime}}{E^{\prime 4}} d E^{\prime} d \Omega^{\prime} \\
& -(1-f) \rho k_{i}^{C} F+(1-f) \rho k_{i \pi}^{c} \frac{1}{4 \pi} \int_{4 \pi} F^{\prime} \alpha^{\prime}
\end{aligned}
$$

where $B=\frac{E^{3}}{(h e)^{3} b .}$

In order to solve the Boltzmann transport equation, the density, temperature, and composition profile must be provided so that the numerical value of the opacity can be found. One approximation is to use the density, temperature, and composition profile from the output of a computer simulation generated using the flux limited difiusion approximation. This should be an acceptable proceedure, to the extent that the two methode of neutrino transport give similar results.

The blocking factors $\left\langle 1-f^{\prime}\right\rangle$ and $(1-f)$ are also evaluated using the value of $f$ obtained from the supernova calculation. The aupernova code used 16 energy bins and the following are taken for the blocking factors:

$$
<1-f^{\prime}>=\frac{\sum_{b i n s}\left(1-\frac{F\left(E^{\prime}, \bar{x}\right)}{\left(\pi E^{\prime 3}\right.}(h c)^{3}\right) E_{i}^{\prime 2} \Delta E_{b i n}^{\prime}}{\sum_{\text {bin }} E^{\prime 2} \Delta E_{\text {bin }}^{\prime}}
$$

and:

$$
1-f=1-\frac{F(E, \vec{x})}{4 \pi E^{3}}(h c)^{3}
$$

One collapse problem, a $15 M_{\odot}$ star, was run with 32 energy bins and weak sensitivity to bin number was found in the calculated spectrum.

Equation (4) is still difficult to solve since it is a differential-integral equation for $F(\vec{x}, \hat{p}, E)$. In the spirit of the proceeding approximations, the spectrum produced by the supernova simulation is used to evaluate the integral involving the electron-positron scattering since, as was shown earlier, it is much less important than the nuclear contribution. Equation (4) can be rewritten as: 
The beams are followed until they come within a distance $\boldsymbol{R}_{\text {min }}$ from the center of the atar. The beams are then picked up on the opposite side. $\boldsymbol{R}_{\text {min }}$ is chowen well inside the neutrinosphere so that in the omitted region, the neutrino are in equilibrium and collisions should not conange $F$. This also helpe to remove sensitivity to the electron-positron scattering opacity, since in this region the neutrinos are degenerate, and the epproximation used for this opacity is not good for degenerate neutrince. The errors introduced by the numerical integration are negligible compared to the uncertainty in the density, temperature, and composition profile used in the opacity conatruetion.

Figures $2 \mathrm{a}$ and $2 \mathrm{~b}$ are polar plots of the angular dintribution for $F_{\nu}$ and $F_{\bar{\nu}}$ (i.e. the electron neutrino and antineutrino diatribution function) at a radius of $270 \mathrm{~km}$ at a tinie late in the collapse of a $25 M_{\odot}$ otar. The dependence on angle is consintent with the interpretation that most of the emission is coming from a region near the surface of the neutrinosphere.

\section{Electron Antineutrinos}

Electron antineutrinos are treated similarly to the electron neutrinos, the only difference being in the opacities. Electron-positron scattering is more important for electron antineutrinos than neutrinos since, while both types scatter from nuclei, antineutrinos are absorbed only by protons, neutrinos only by neutrons, and the value of $Y_{P}$, the proton fraction, can drop as low as .1 in the region near the neutrinosphere. Therefore, the approximate form for the electron-positron scattering term in the Boltzmann equation will have a greater effect on the electron antineutrino spectrum than on the electron neutrino spectrum. However, the supernova code includes the effects of electron-positron scattering with a more consistent approximation than that used in the Boltzmann equation, and relatively good agreement is obtained with the flux limited diffusion calculation and the calculation done with the Boltzmann equation.

\section{Mu Tou Neutrinos and Antineutrinos}

Mu tau neutrinos and antineutrinos (which can be treated as four essentially identical neutrinos when $C_{V}=1$ and $C_{A}=.5$ for $e y_{e}$ scattering) are scattered but not absorbed by nucleons. The electron-positron opacity is now comparable to the baryon opacity. No attempt .0 solve for a spectrum of these neutrinos with the Boltzmann equation is made since the electron-positron scattering opacity used is only approximate. However, with good 
agreement of the electron antineutrino spectrum calculated with the mpernore code and the Boltzmann equation, and the fact that electron-foritron scattering can be an important source of opacity for electron antineutrinos, the supernove code calculated mu tau opectrum is done adequately.

\section{SUMMARY}

Figure 3(a-e) show comparasions of the electron neutrino npectrum calculated with the fux limited diffusion approximation and with the Boltzmann equation. Figure 4(a-e) show the electron antineutrino spectrum as calculated by both methoda. Five times are chosen during the evolution to show general trends. Figures 3 a and 4 a are .1 eecond after bounce, while figures $3 b$ and $4 b$ are just before the explosion. Figures $3 c$ and $4 c$ are at the explosion time. These three times show the most discrepancy between the two methods, and are all taken when there is unsteady motion in the atmosphere. Figures 3d,3e,1d,and 1 show times after explosion, when matter motion is fairly steady on one second timescales. Here agreement is very good. The $25 M_{\odot}$ model $C$ progenitor atar, as listed in Wilson et al. (1985), is the stellar model that produced the spectra seen in figures 3 and 4.

Good agreement is found between the spectrum as calculated by the Boltzmann equation and by the flux limited diffusion approximation, as average energies calculated using both spectra were within $20 \%$ of each other. The luminosities, however, were in better agreement, between 5 to $10 \%$. The peak discrepancies are at the times which had the most motion in the atmospheres. (If the flux from the Boltzmann equation were actually fed back into the evolution equations for the star, the system would adjust so as to lower this discrepancy. For example, too high a flux would lower the temperature, thereby bringing down the flux.)

One weakness in the above calculation is in the electron-positron scattering cross-section. The form used is certainly not good in the region where the neutrinos are degenerate, but at this time the blocking factors help to reduce the importance of the electron-scattering cross-section. In fact, inside the neutrinosphere near the star center, where electrons are very degenerate, the agreement between the two methods of calculation is only fair, but the spectra and flux exiting the neutrinosphere is within the estimates given above.

It must be remembered that the supernova code finds a spectrum by the time integration of the flux limited diffusion equation, while the Boltzmann equation solved in this chapter assumes the static limit. The flux limited distribution function contains a history of the supernova evolution and when the atmosphere is unsteady, disagreement between the two 
methods of calculation should be expected. Even though $v / c \leq .2$ (we Chapter III), the time rate of change of $v / c$ and the distribution function can be important in determining the spectrum. In fact, at $t=.4695 \mathrm{sec}$, or the time figures $3 \mathrm{a}$ and $4 \mathrm{a}$ are taken, the atructure of the sta: was frozen, and the supernova flux limited diffusion subroutines were used to evolve the neutrinos until a steady state situation was reached. This test resulted in improved agreement of the spectra and luminosities. However, repeating this proceedure at $t=.7835$ sec. (figures $3 \mathrm{~b}$ and $4 \mathrm{~b}$ ) failed to improve agreement.

Some further results concerning the neutrino spectra and luminosity for the $25 \mathrm{M}_{\odot}$ model C star (see Wilson et al. (1985)) is now presented. Figures 5(a-e) in this chapter show the sum of the spectra for the mu, tau neutrinos and their associated antineutrinos, as calculated by the flux limited diffusion approximation. Five times during the collepoe are chosen, and correspond to the same times shown in figures 3 and 4. Figures $5(\mathrm{f}-\mathrm{h})$ show the time integrated spectrum for the electron neutrinos,electron antineutrinos, and the mu-tau group of neutrinos. Figures $5 \mathrm{i}$ and $5 \mathrm{j}$ show the electron neutrino and electron antineutrino luminosity versus time. They are similar, except that the electros capture burst seen in the electron neutrino luminosity during collapse is not seen in the electron antineutrino luminosity (there is no corresponding process). Figure 5k shows the electron neutrino, electron antineutrino and one of the mu tau group of neutrinos luminosity superimposed onto the same graph. Figure 51 shows the strueture of the electron capture burst on a magnified time scale. Figures $5 \mathrm{~m}$ and $5 \mathrm{n}$ show average neutrino energies for the various neutrino types as a function of time. Since mu and tau neutrinos and antineutrinos only scatter from baryons, their mean free path tends to be longer than for electron neutrinos and antineutrinos, which are emitted and absorbed by baryons, as well as scattered. This means that average energies for the mu tau group of neutrinos are generally larger than the electron neutrinos and antineutrinos. This is the case since the mu tau group of neutrinos can escape from the star at a smaller radius, where the temperature is higher than at the radius where electron neutrinos and electron antineutrinos escape. After explosion, however, due to the drop in the proton fraction near the outer part of the proto-neutron star (see Chapter $V$ ), the average electron antineutrino energy increases to about the value of the mu tau group of electrons. There are few protons to participate in emission and absorbtion reactions with $\bar{\nu}_{e}$. Therefore, the electron antineutrino has a mean free path close to one of the mu tau group of neutrinos. Figures $6(\mathrm{a}-0)$ show the time integrated neutrino spectra for some of the 
other stars studied in Wilson et al. (1985). Figures $5(2-0)$ show the luminosities obtained from some of the stars studied in Wilson et al. (1985). The burat signal from electron capture is very similar for all iron core collapee driven supernova, since it is produced by the deleptonization of approximately the inner $.6 M_{\odot}$ of these collapoing atars. Figure 8 shows the curve of binding energy versus gravitational mass of a neutron star used to obtain the remnant masses for the supernovae found in Wilson et al.(1985). This bindins energy is also used to calculate the total luminosity in neutrinos that must be produced by the remnant as it becomes a cold neutron star. 


\section{Chapter IV References}

Tubbs, D.L., and Schramm, D.N. 1975, Ap. J., v201, 467.

Wilson, J.R., Mayle, R., Woosley, S.E. and Weaver, T.A. 1985,

XII Texas Symp. Rel. Ap., ir press 


\section{Chapter IV Figure Caption:}

Fig. 1 Electron neutrino scattering crosesection constructed using the results of Tubbs and Schramm (1975). T, $\mu_{e}$, and the neutrino energy are all measured in Mev. Crosssections are measured in units of $10^{-41} \mathrm{~cm}^{2}$.

Fig. 2 Angular distribution of the electron neutrinos with energy $8 \mathrm{Mev}$ and angular distribution of tine electron antineutrinos with energy $16 \mathrm{MeV}$ at a point $270 \mathrm{~km}$ from the star center at late times. The neutrinosphere is inside the point where the angular distribusion is shown. The star is the 25 model C (see Wilson et al. (1985)).

Fig. 3 Comparasion between the flux limited diffusion approximation calculated spectrum (FLD) and the Boltzmann equation (BE) for the neutrinos at 5 different times. The star is the 25 model C. Integrating the area under a curve gives the luminosity in Mev/sec, or $1.6 \times 10^{-6} \int l d e=L \frac{\mathrm{er} q}{\mathrm{aec}}$.

Fig. 4 The same comparasion is made for the electron antineutrinos as was made for the clectron neutrinos (see fig. 3 caption).

Fig. 5(a-e) The combined spectrum from the 4 members of the mu tau group of neutrinos as calculated by the flux limited diffusion approximaton. The five times chosen agree with the times in figures 3 and 4.

Fig. 5(f-h) Time integrated spectrum, taken at three different times for the electron neutrinos and antineutrinos and for the 4 members of the mu tau group. The star is the 25 Model C.

Fig. 5(i-1) Luminosity versus time curves for for the electron neutrino, electron antineutrino, and one of the members of the mu tau group, call it the muon neutrino. Note the sharp peak of electron neutrinos at the early times. This is the burst from electron capture. For ease in seeing other structure, the electron capture burst is drawn one half it's act" 1 height. This does not apply to figure 51 which is a magnified view of the structure of the electron capture burst.

Fig. $5(m-n)$ The time dependence of the average neutrino energies seen in the collapse of 25 Model $\mathrm{C}$ star. Fig $5 \mathrm{n}$ shows the comparasion between the average energy as given by the fux limitied diffusion, denoted by the subscript $C$, and by the Boltzmann equation generated spectrum, denoted by the subscript $\mathrm{S}$. Figure $5 \mathrm{~m}$ shows the effect of redshift on the average energies.

Fig. 6 Time integrated spectra from many of the stars studied in Wilson et alf (1985). 
Fig. 7 Luminosities from many of the stars studied in Wilson. et al. (1985)

Fig. 8 Neutron star binding energy as a function of gravitational mass. This curve is used to find the total amout of energy that must be radiated away in neutrinos as a hot neutron star cools to a final cold state. 


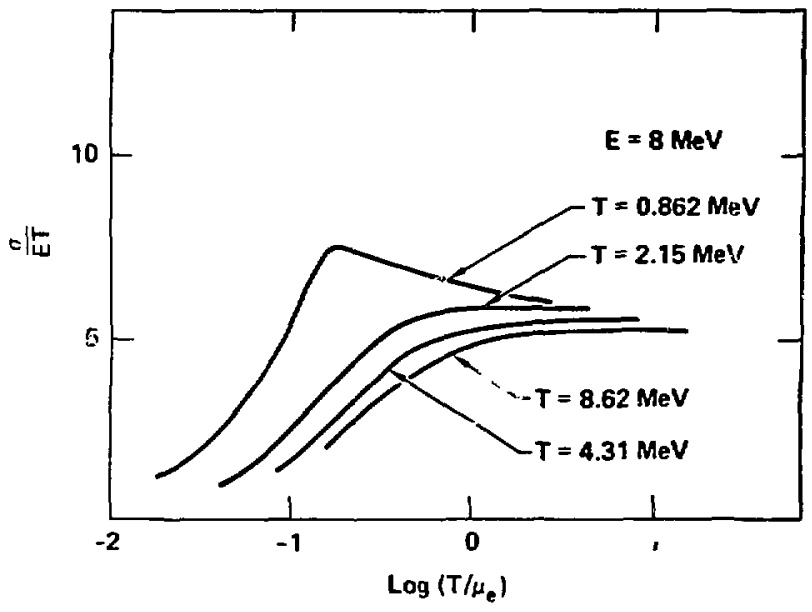

FIGURE la 


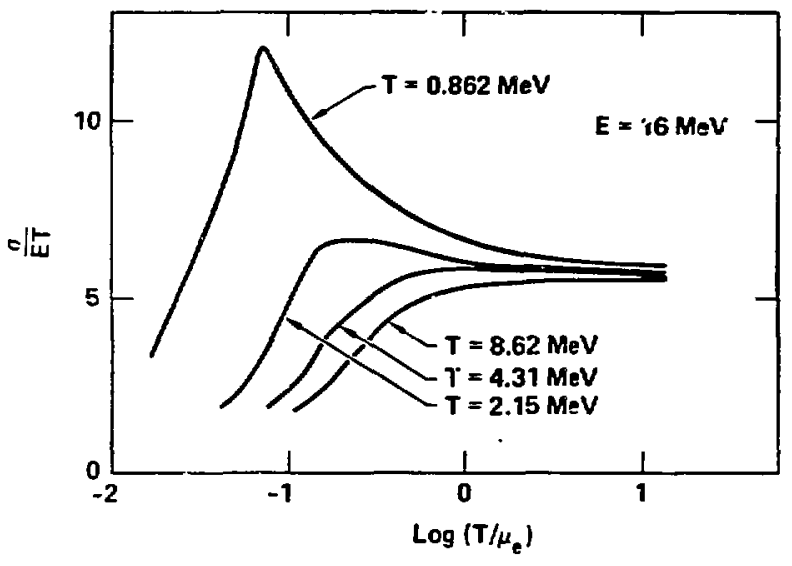

FIGURE Ib 


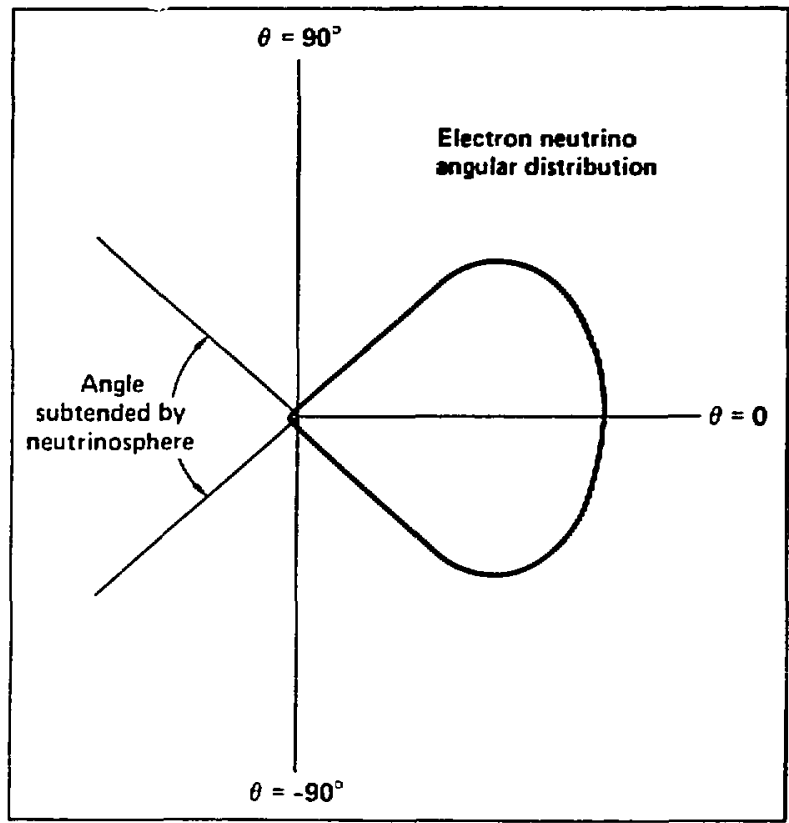

FI Gบกั 2 a 


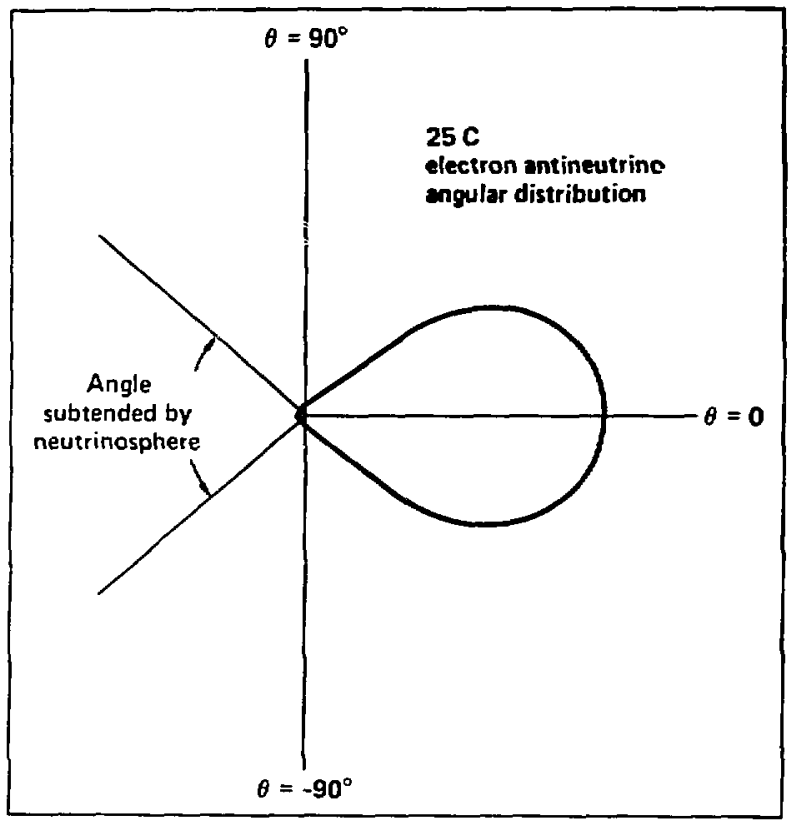

FIGURE 2b 


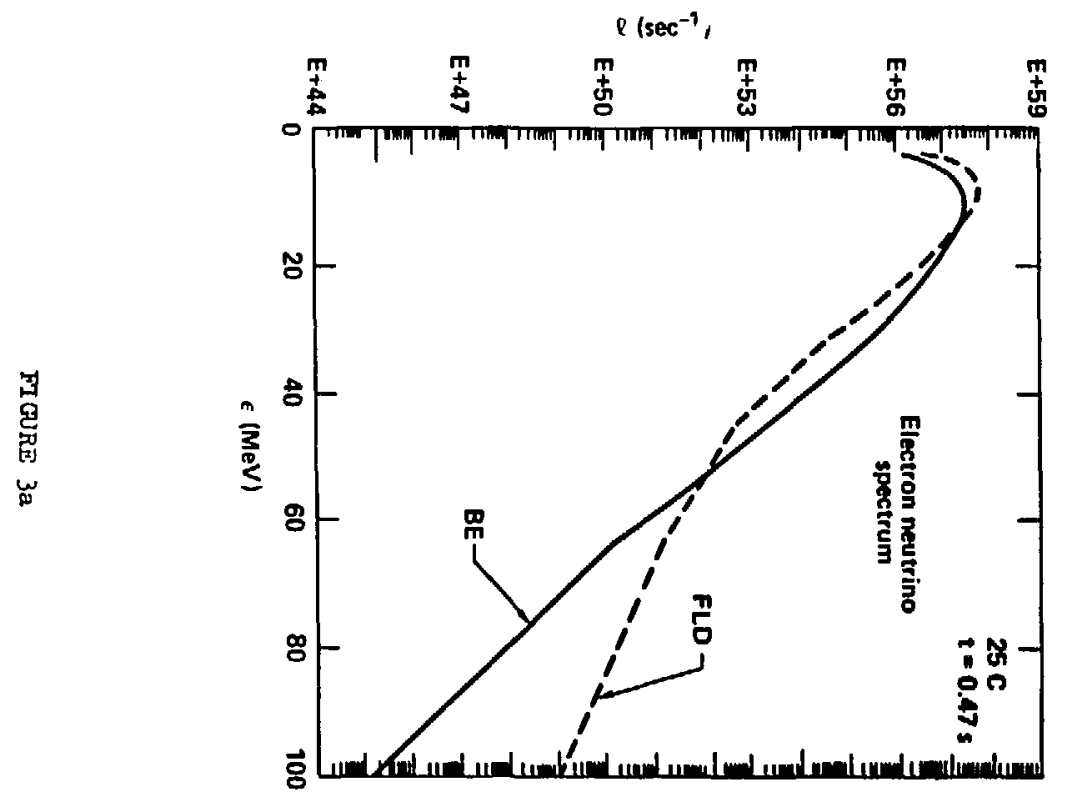




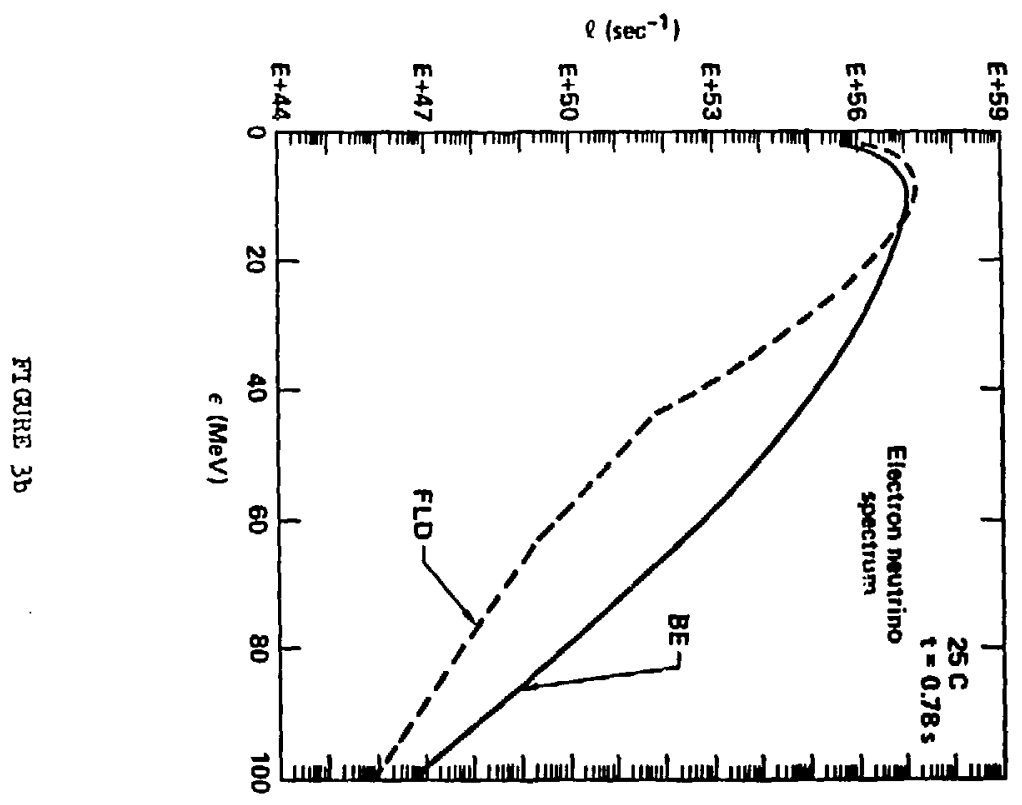




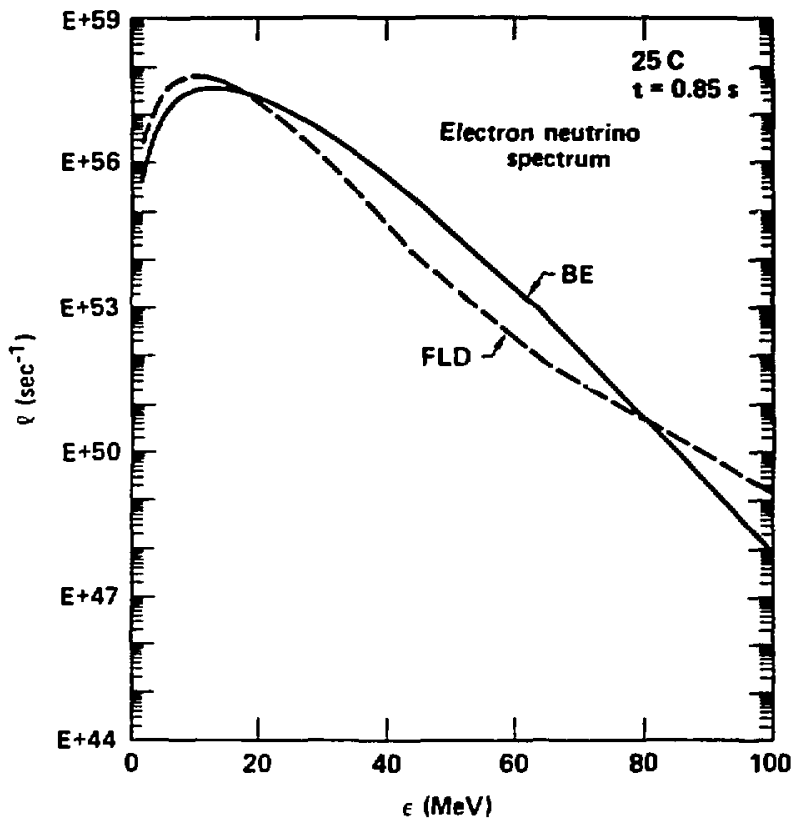

FI GJRE $3 \mathrm{C}$ 


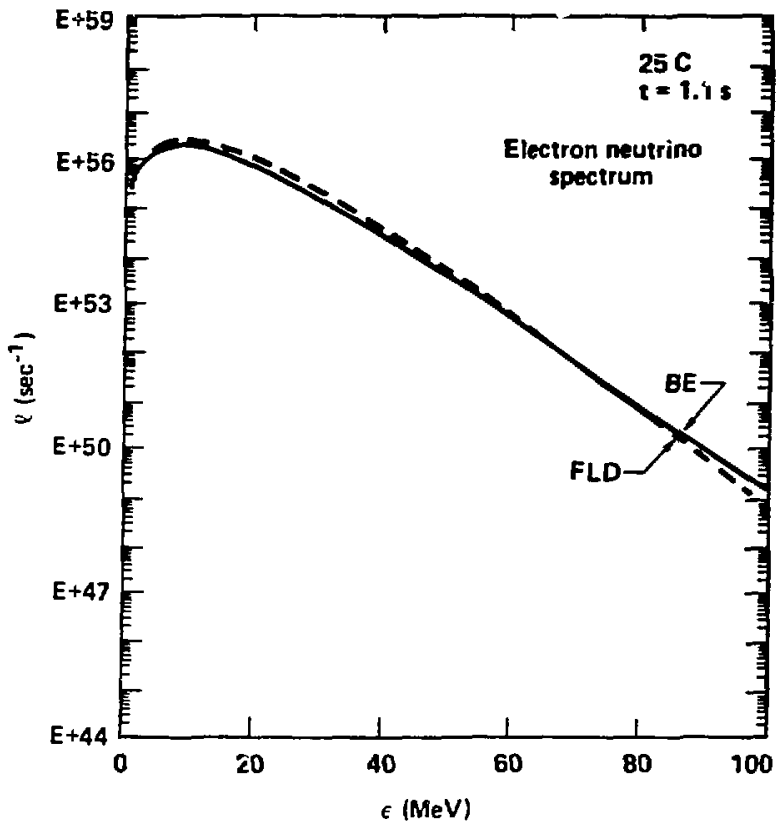

FI GRE 3d 


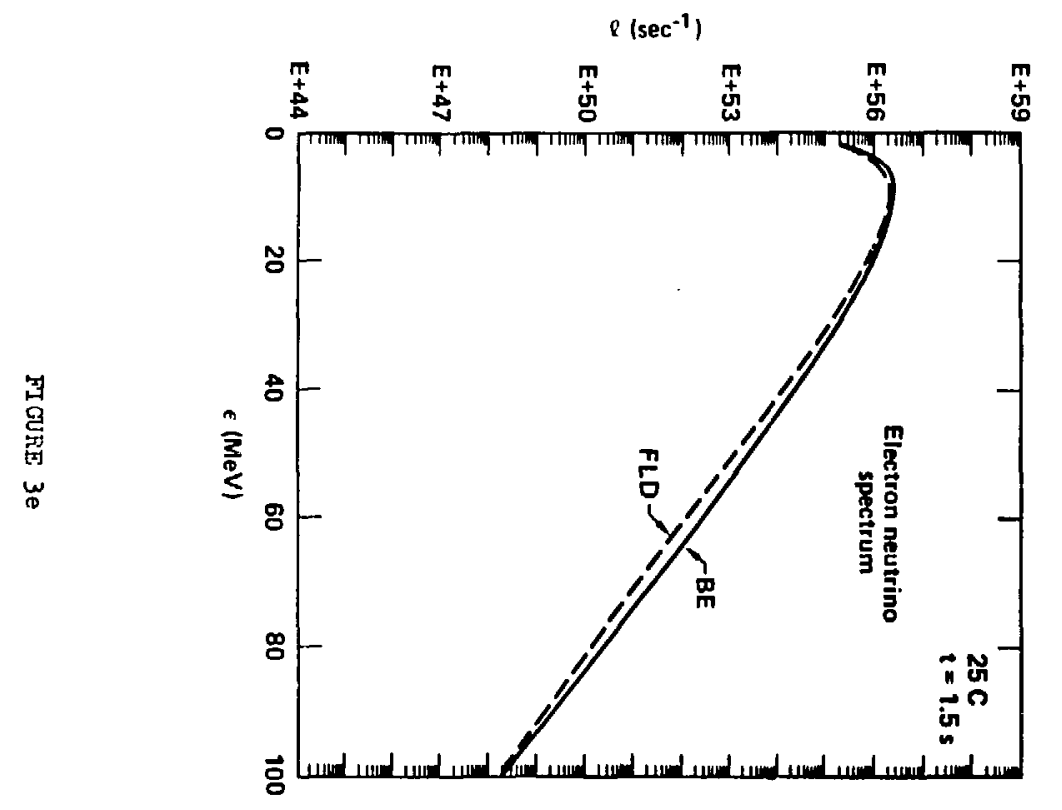




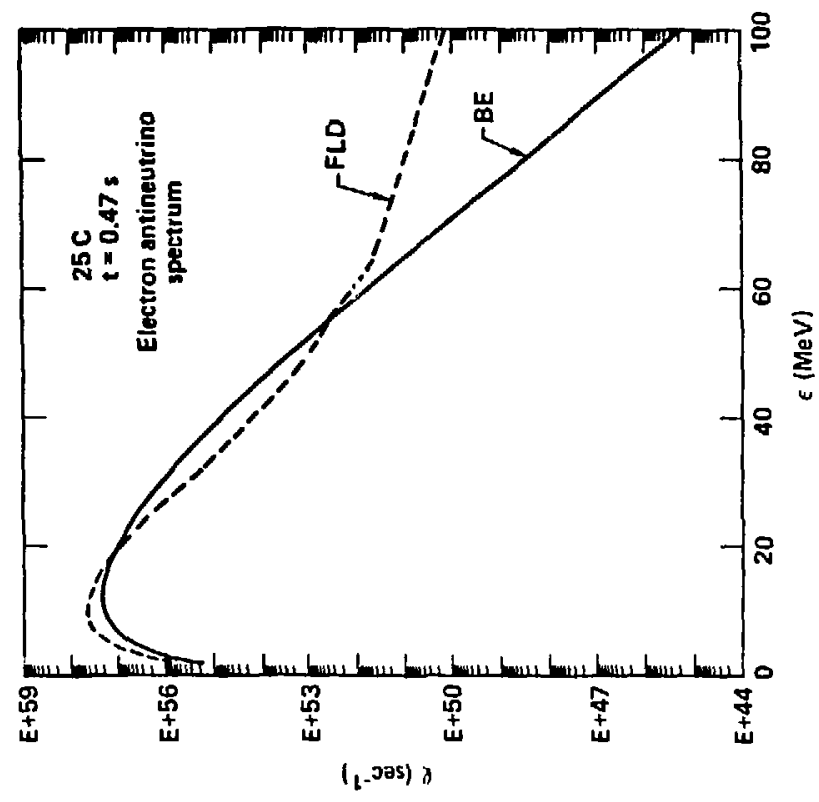




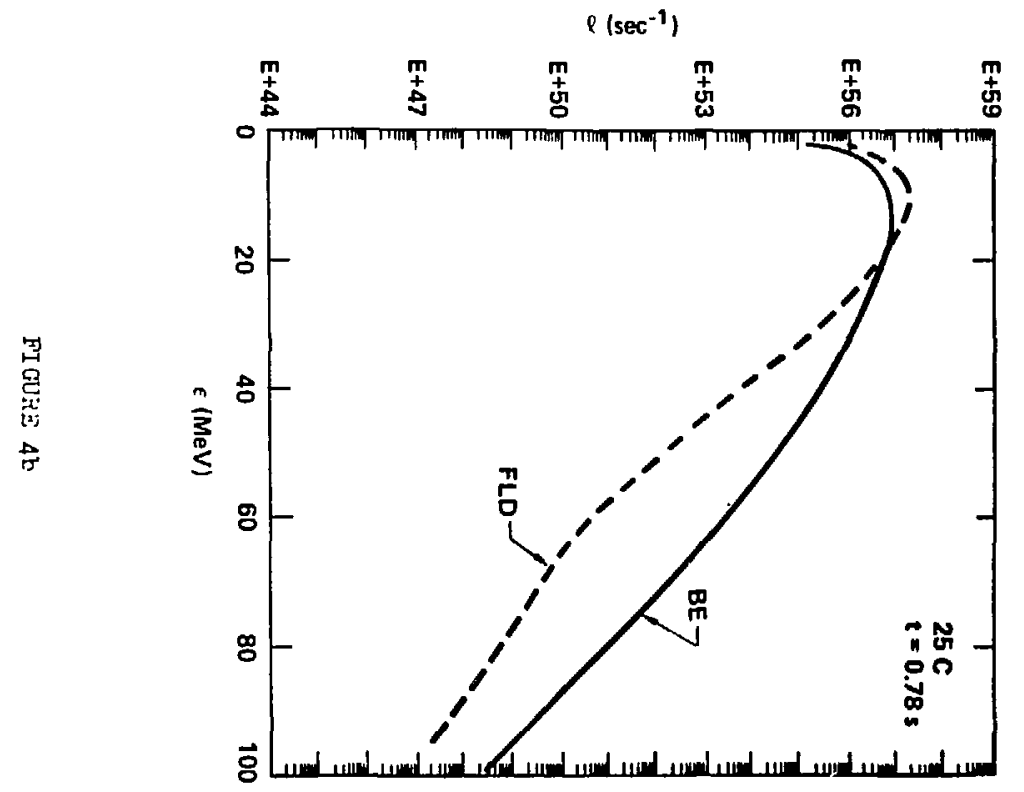




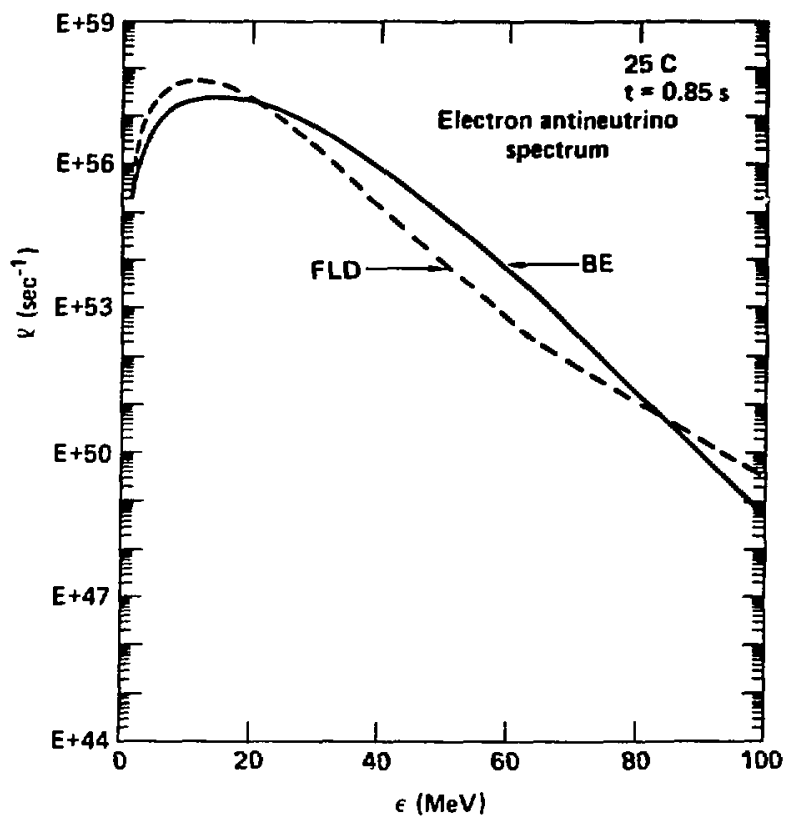

FI GUKE $4 \mathrm{C}$ 


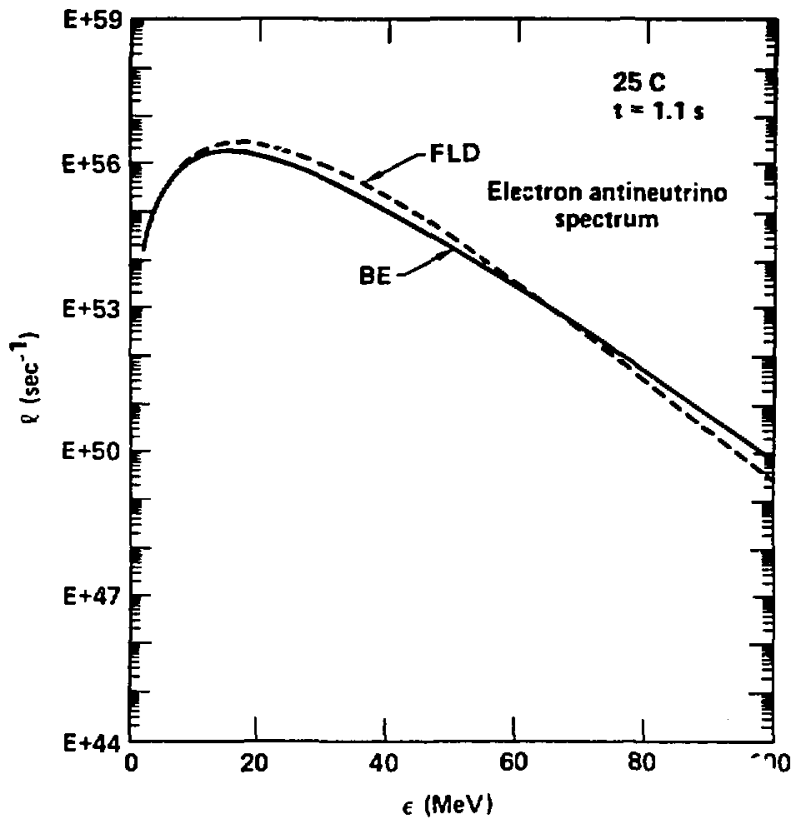

FI GURE 4d 


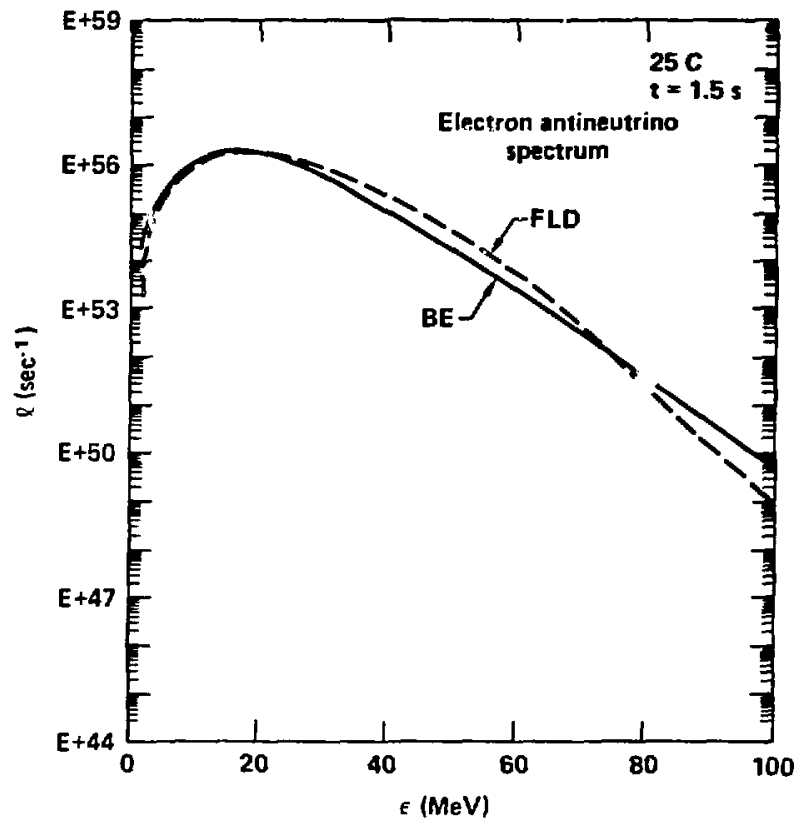

FIGJRE 4e 


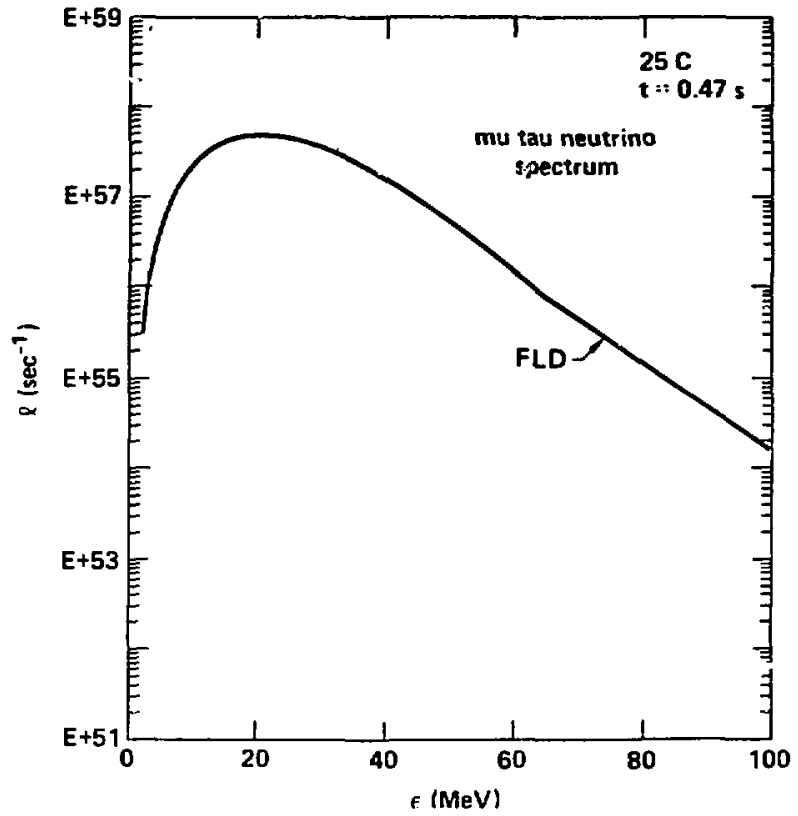

FI GURE $5 a$ 


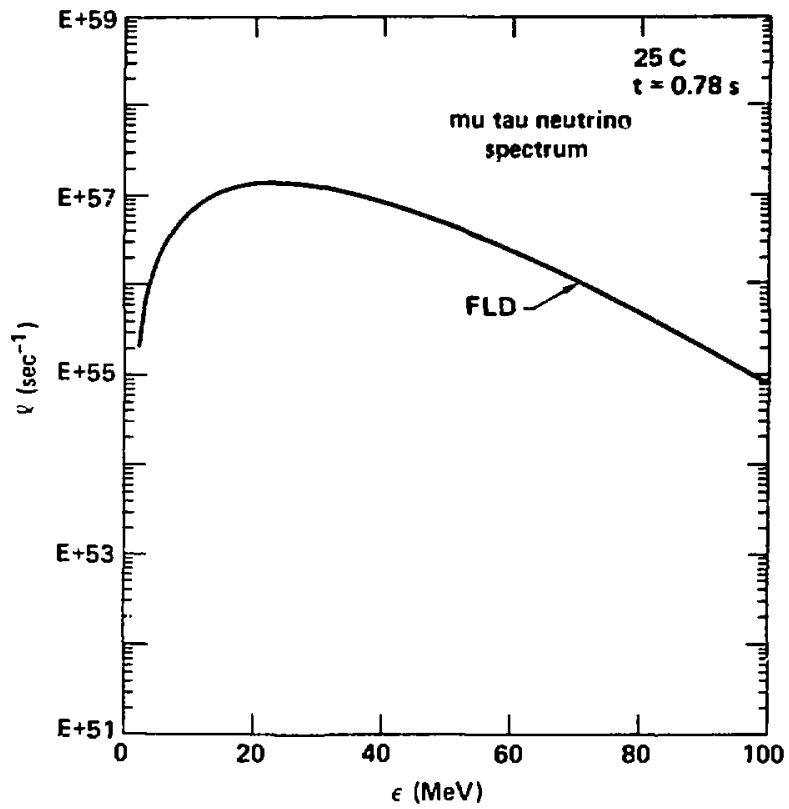

FI GURE 5b 


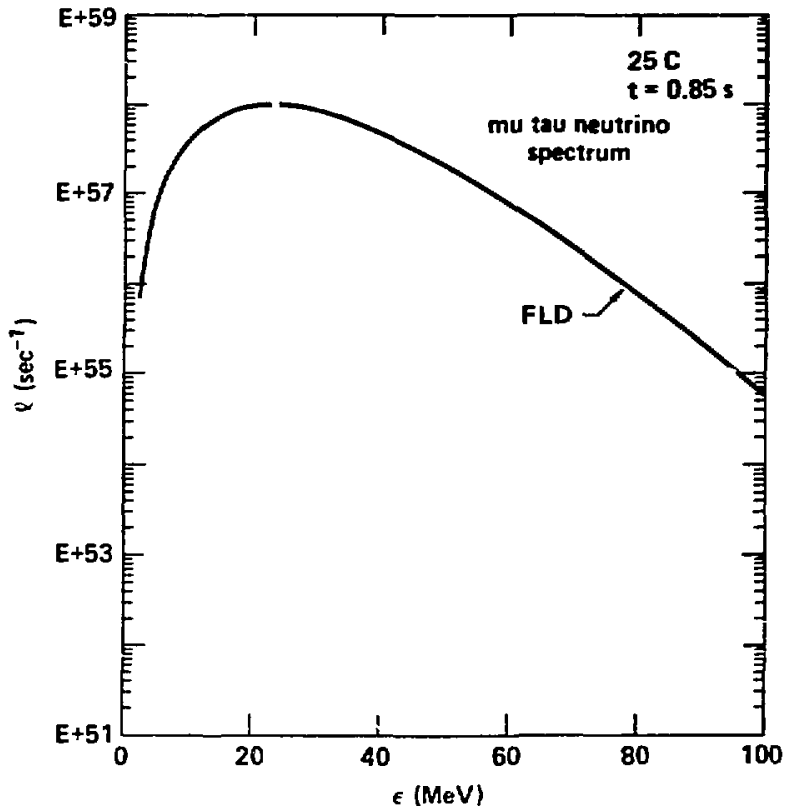

FI GURE $5 c$ 


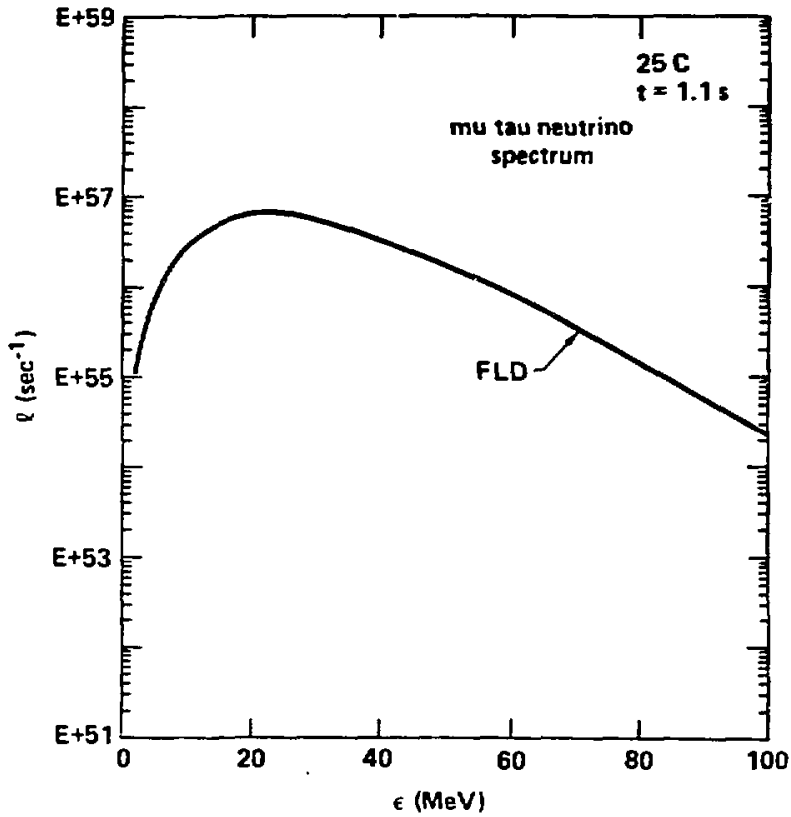

FIGURE $5 d$ 


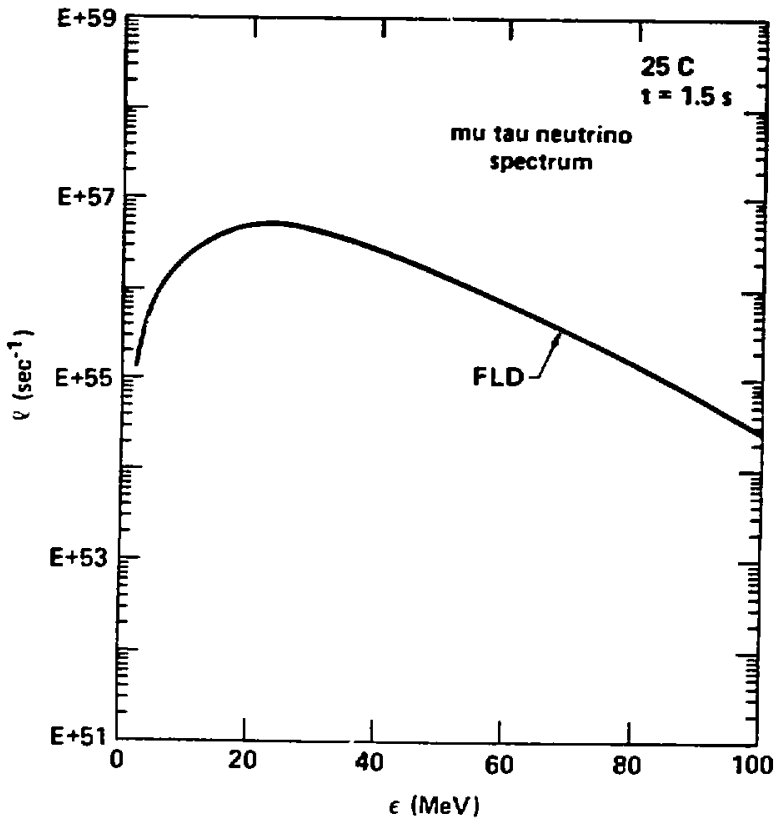

FI LURE $5 e$ 


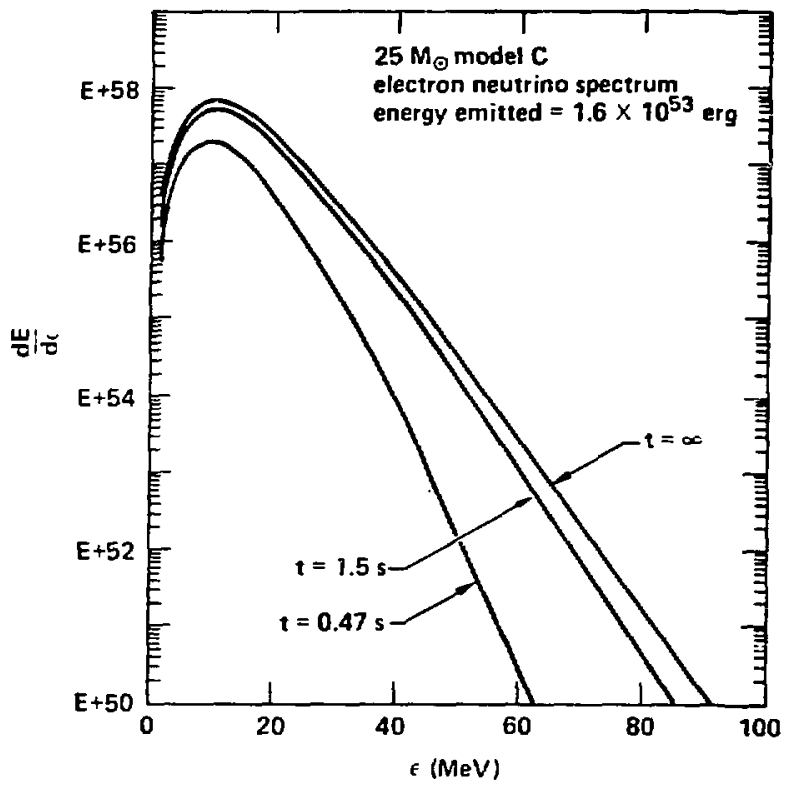

FI GUPE $5 f$ 


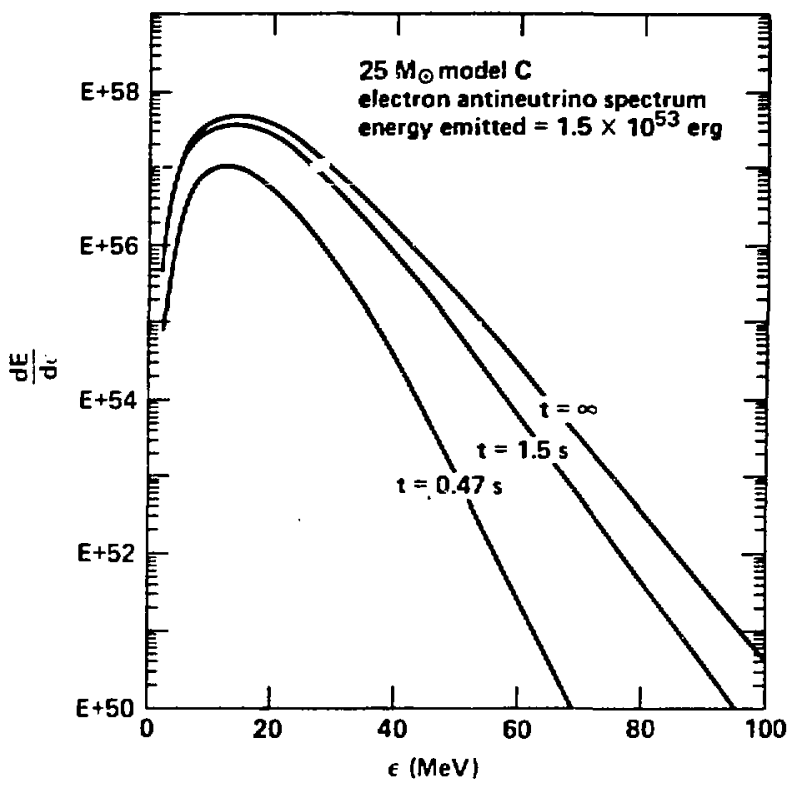

FI GURE $5 g$ 


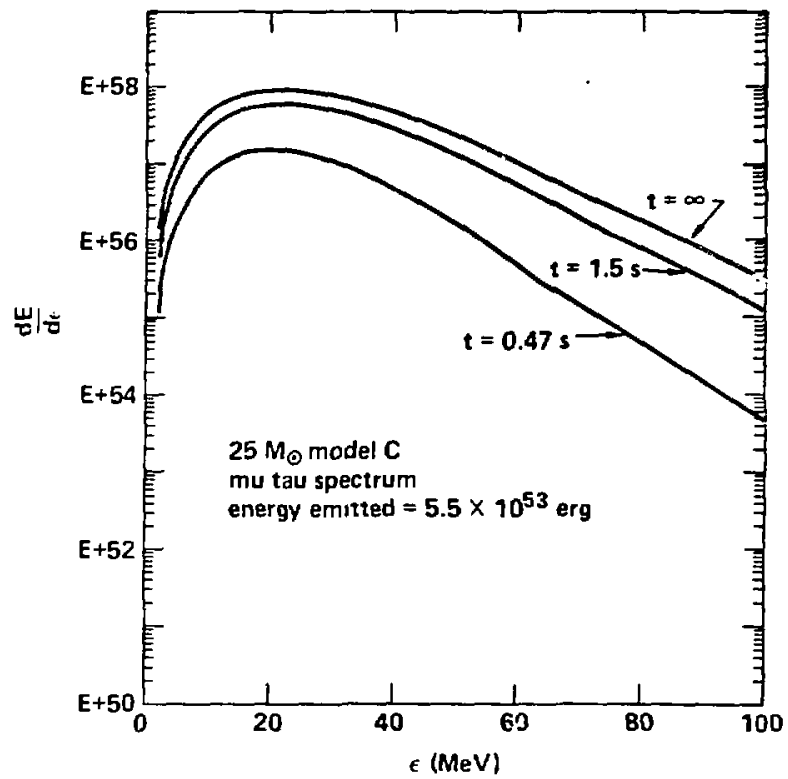

FIGURE $5 \mathrm{~h}$ 


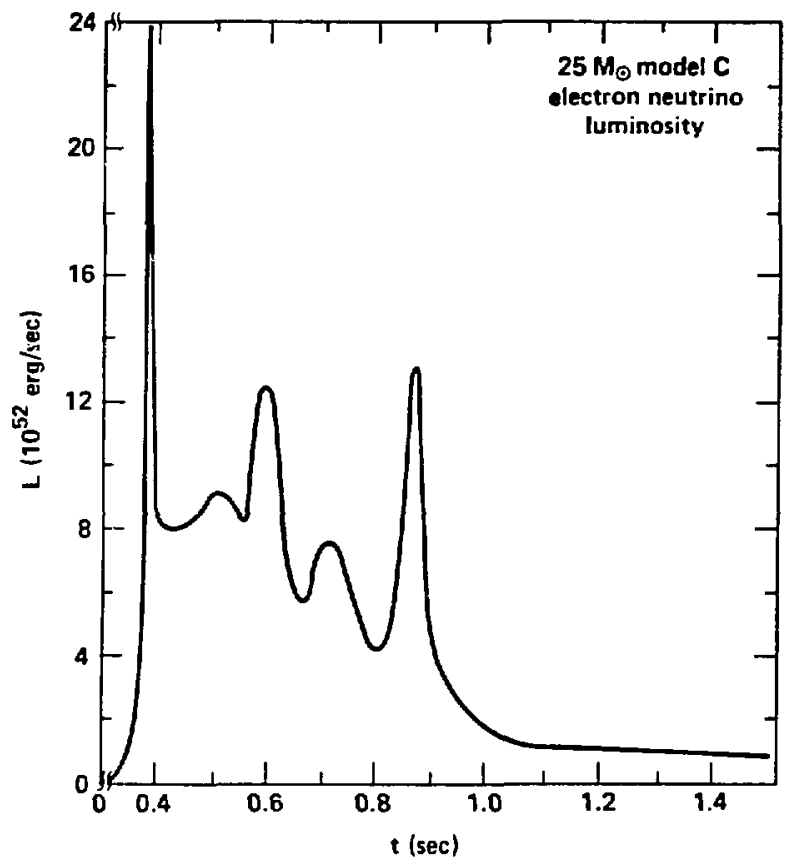

F'I GU:I $5 i$ 


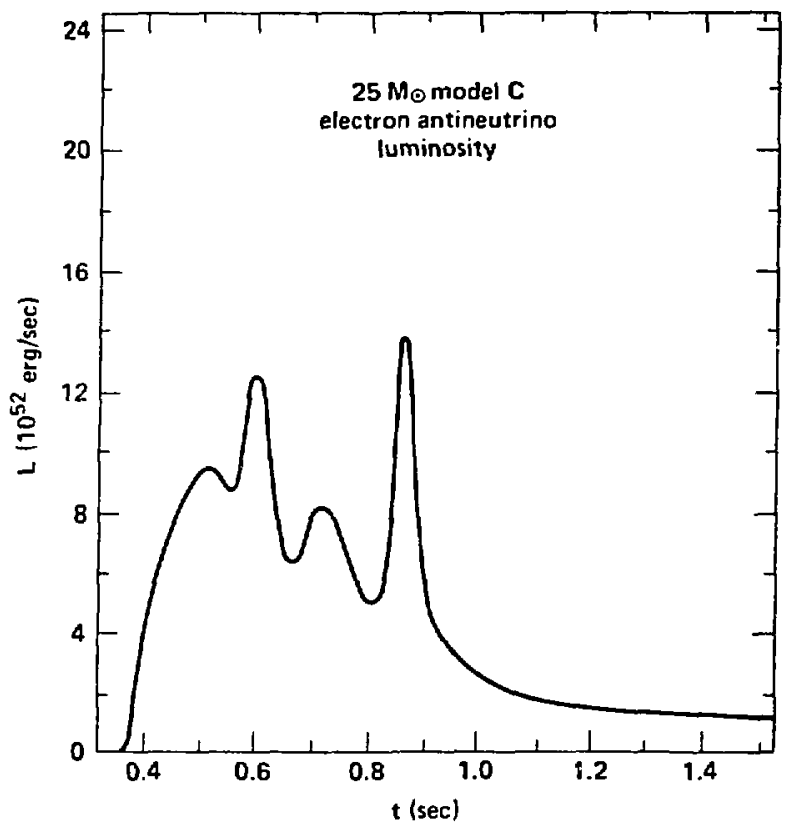

FIGURE $5 j$ 


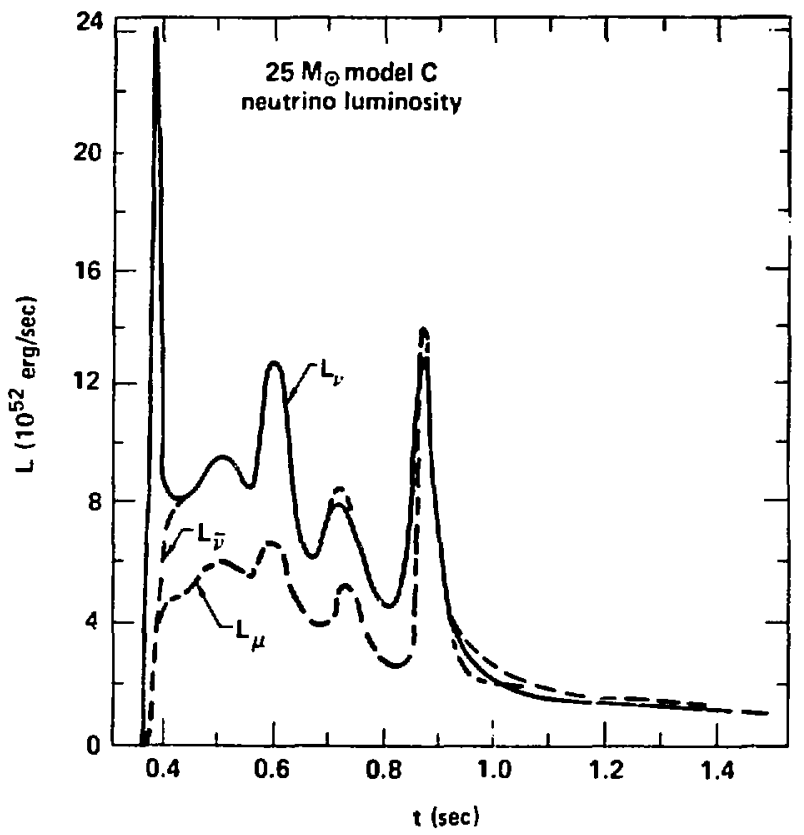

FI GURE $5 k$ 


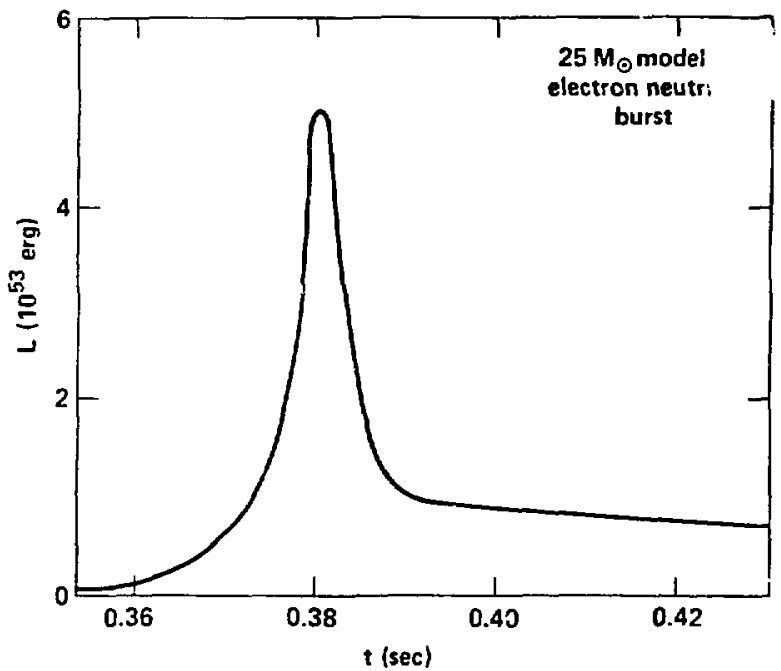

FIGURE 51 


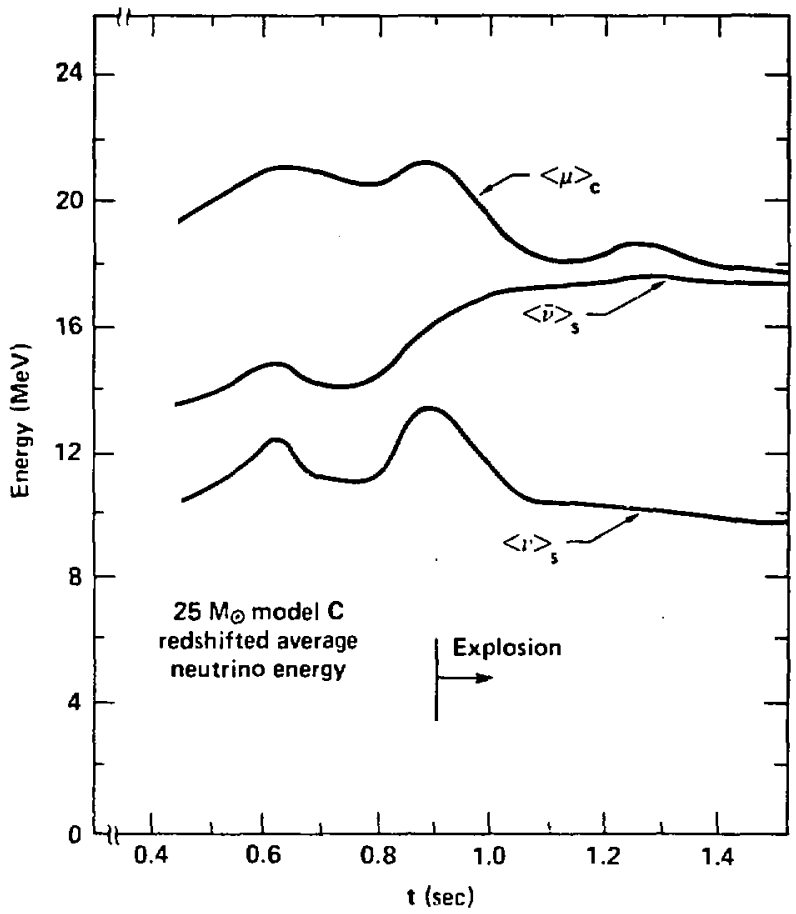

FICU:S $5 \mathrm{~m}$ 


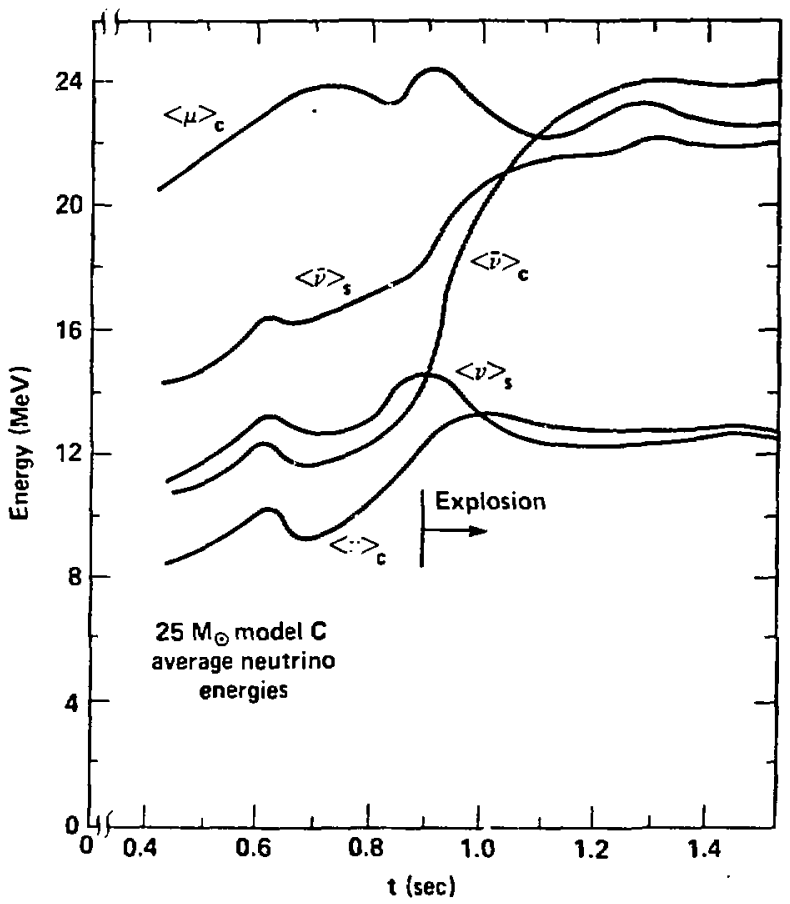

FIGURE $5 n$ 


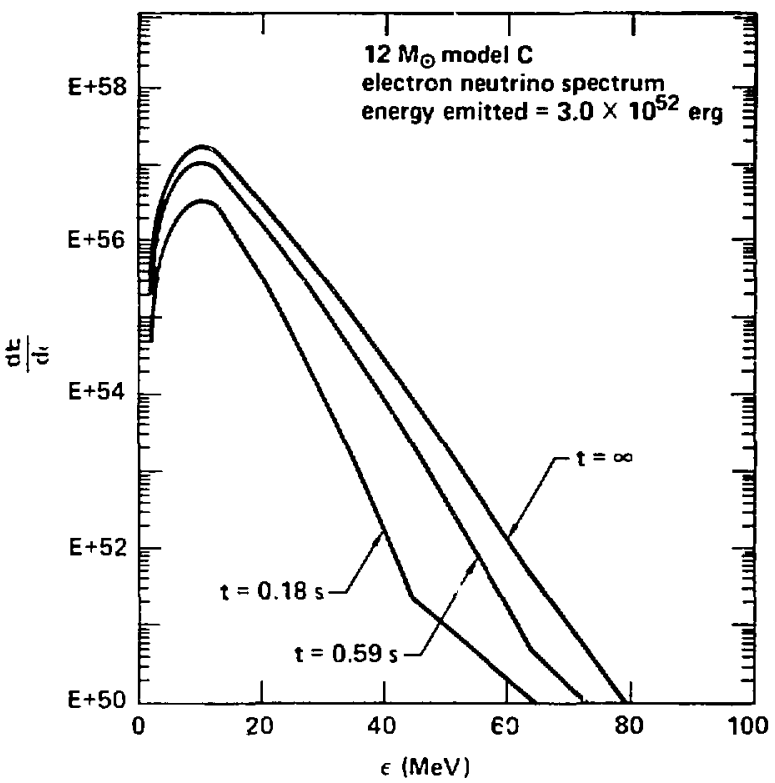

FI GURE $6 a$ 


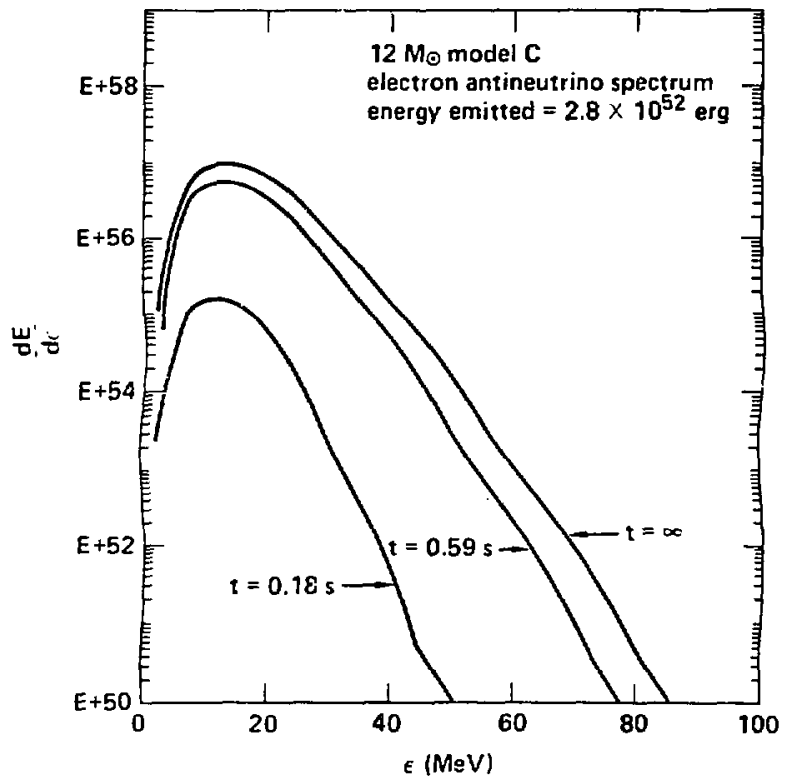

FICJRE 6b 


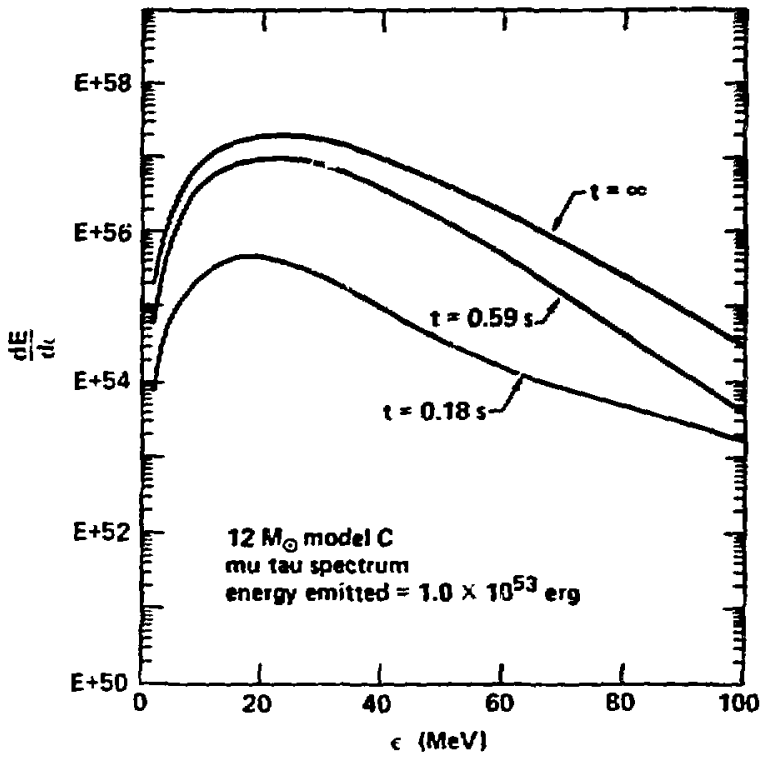

FIGU:E 6c 


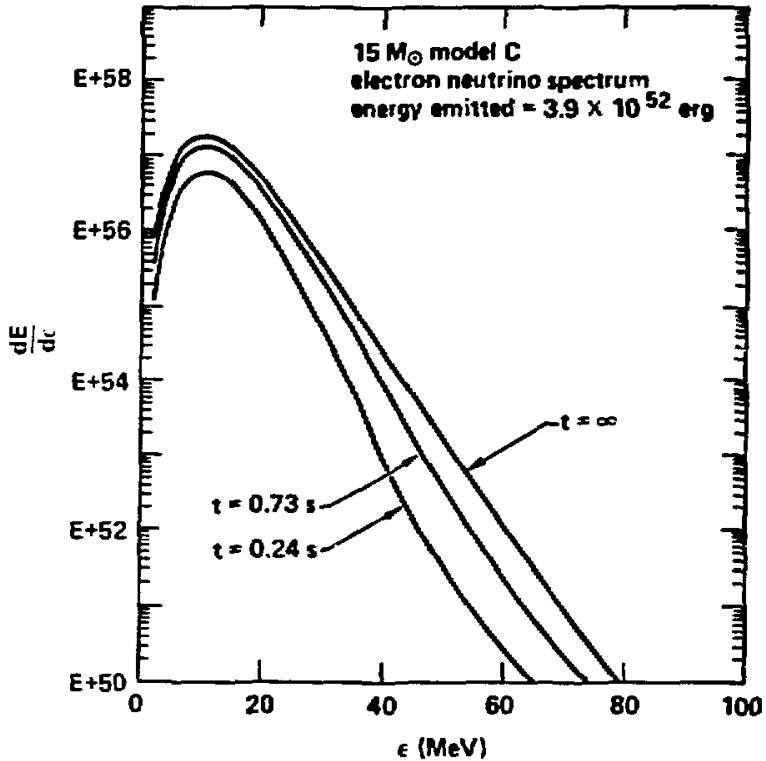

FIGURE 6d 


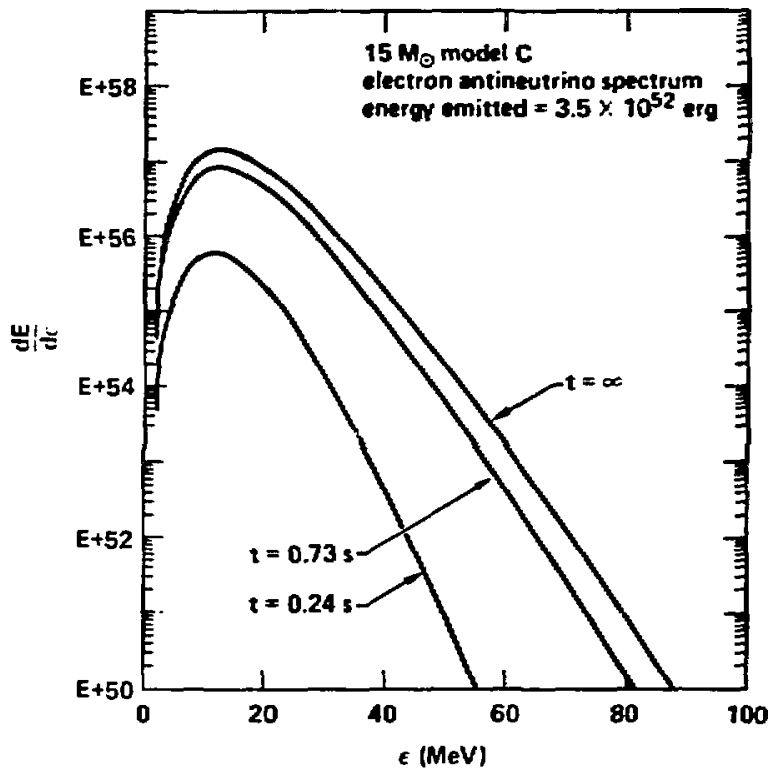

FIGIRE $6 \mathrm{e}$ 


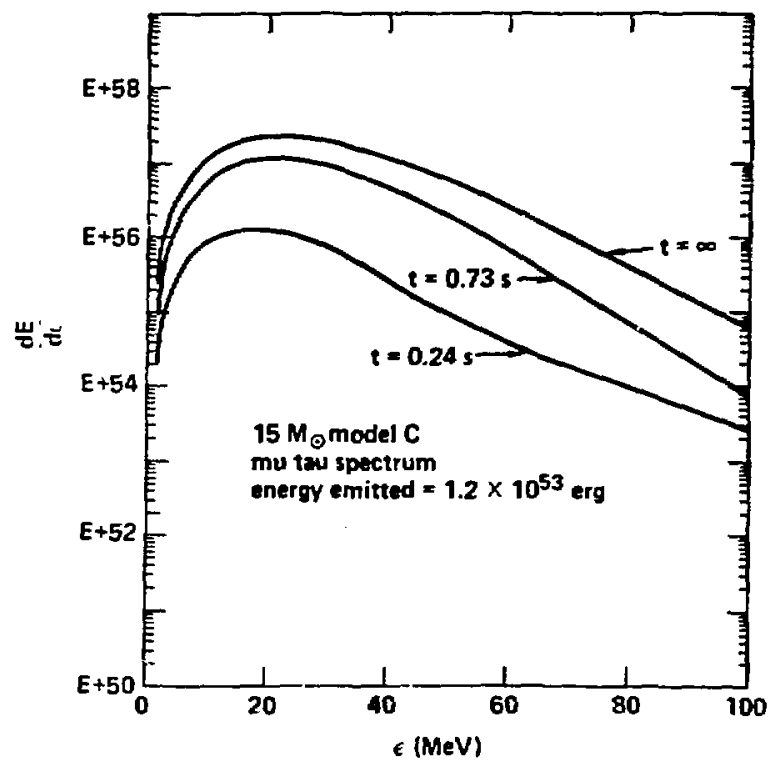

FIGURE $6 f$ 


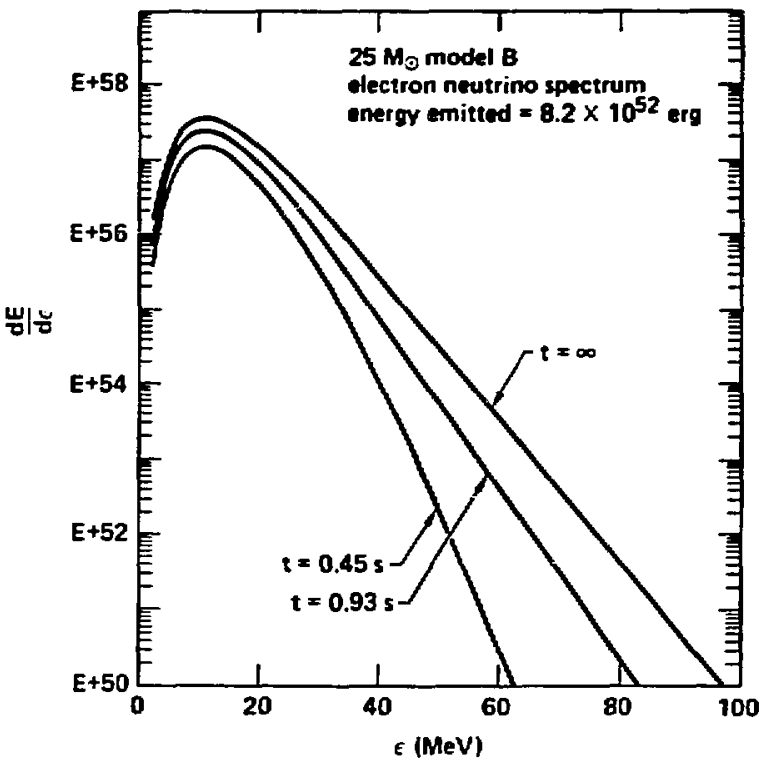

FI GUAE $6 \mathrm{C}$ 


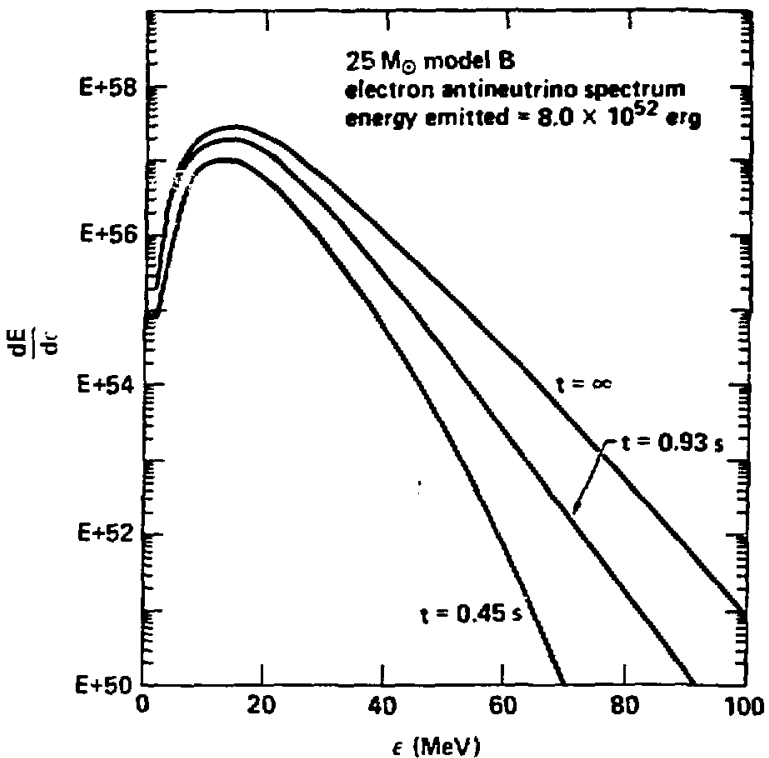

FuGize 6r 


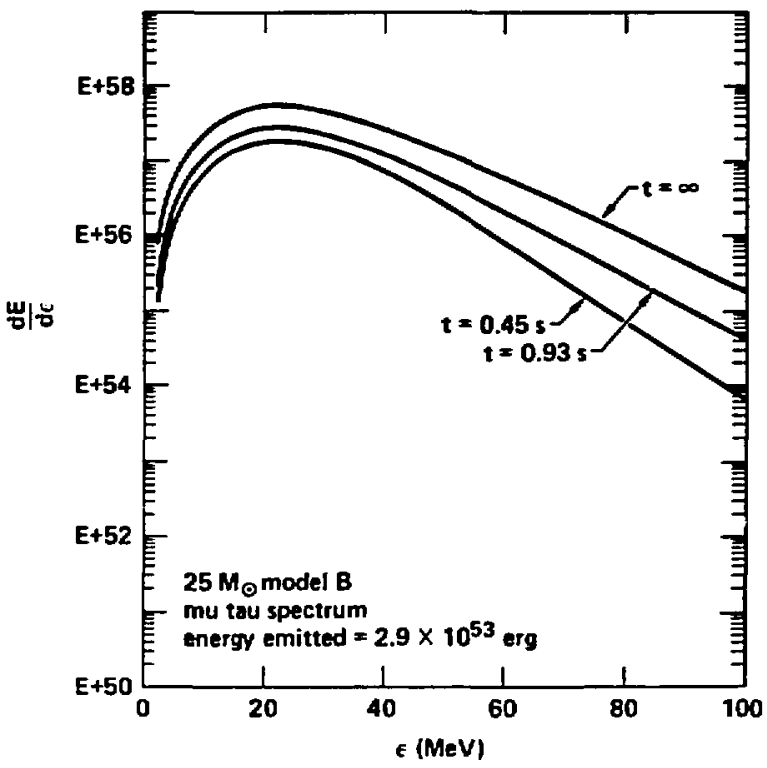

FIGURE $6 i$ 


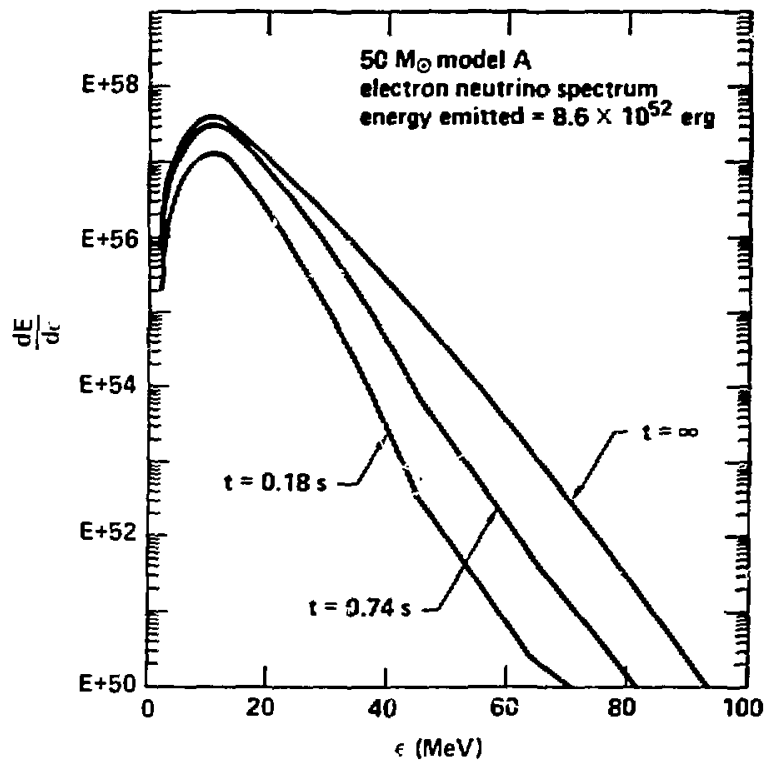

FI GURE $6 j$ 


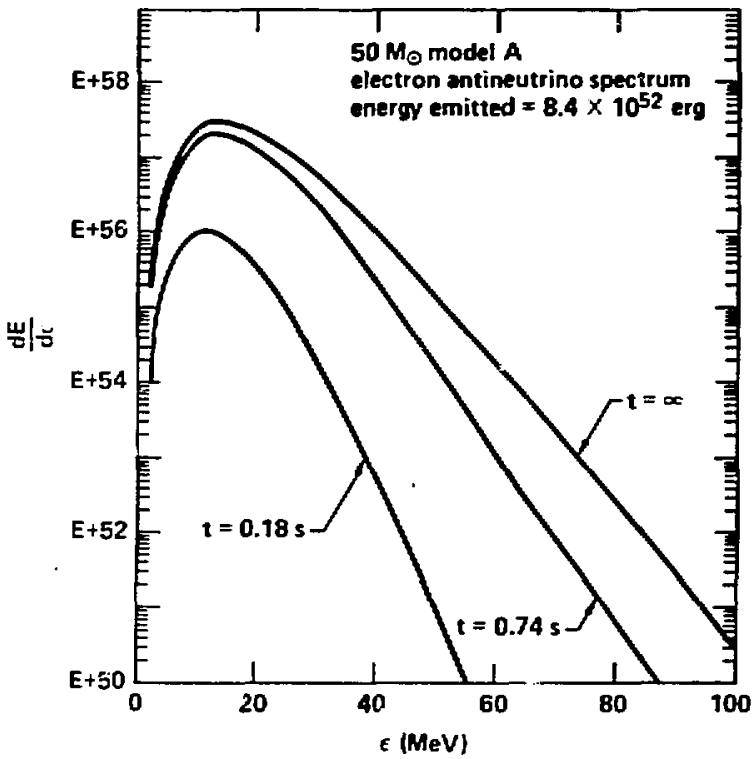

FIGURE $6 k$ 


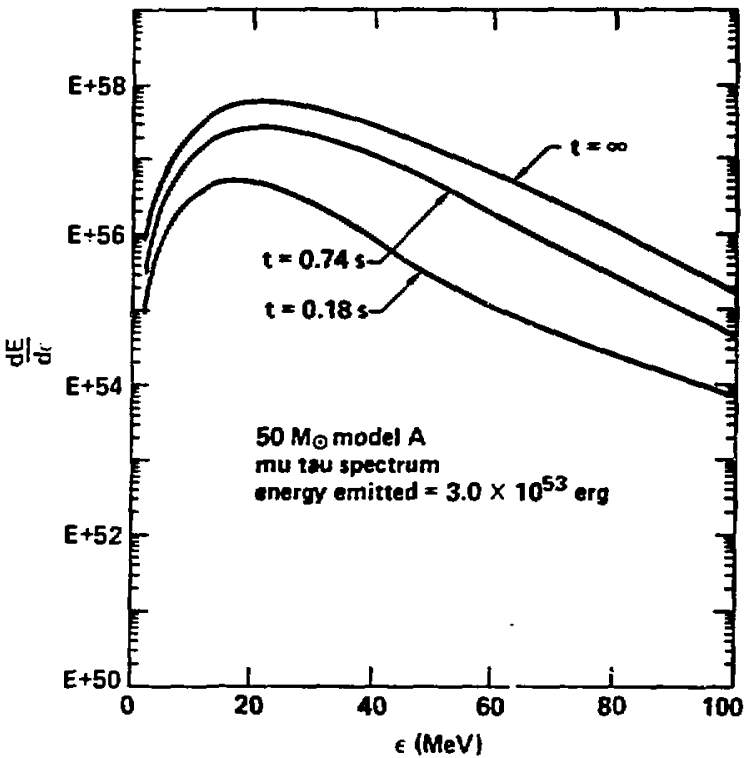

FIGIRE 61 


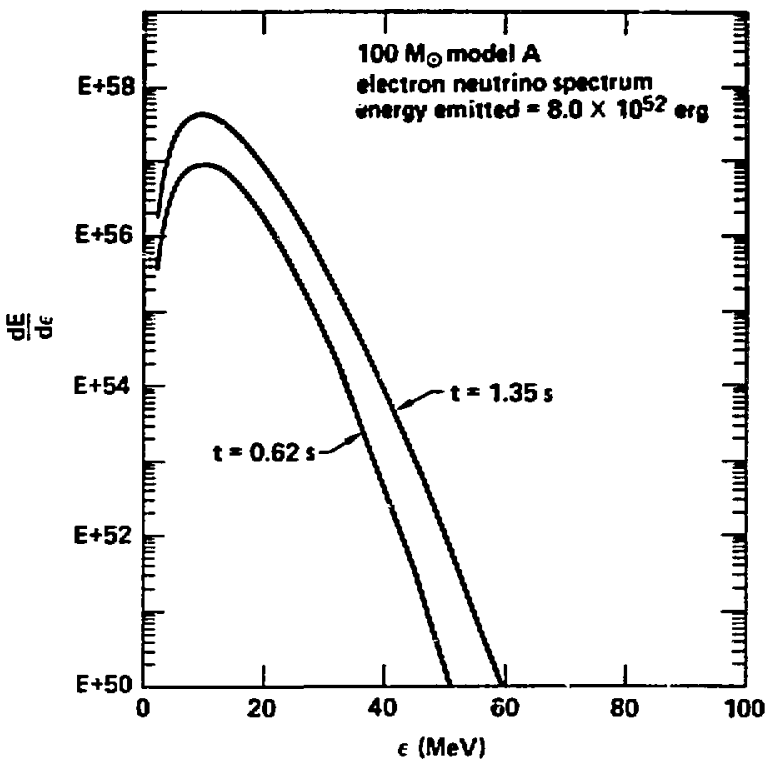

FIGLRP $6 \mathrm{~m}$ 


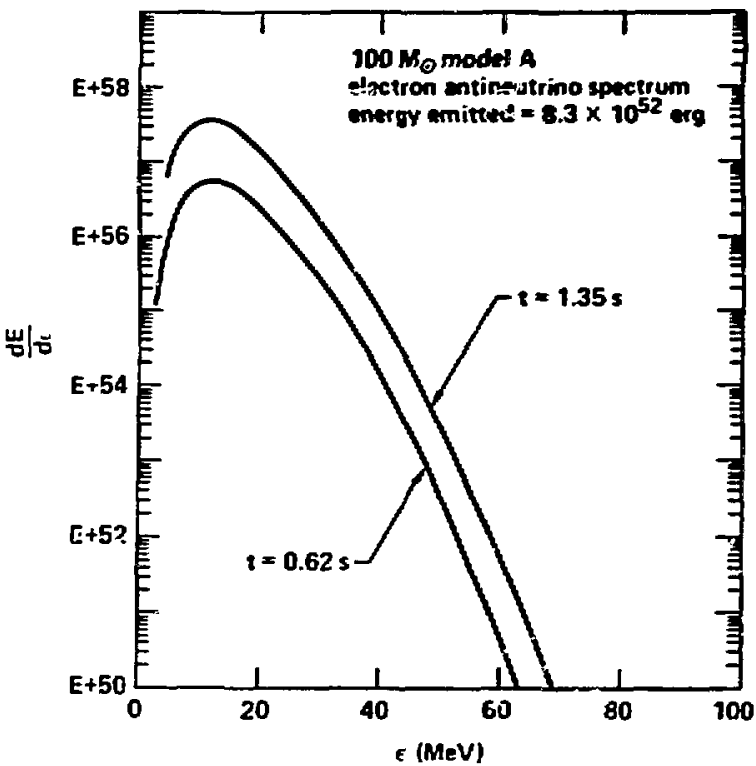

FIGU: 6n 


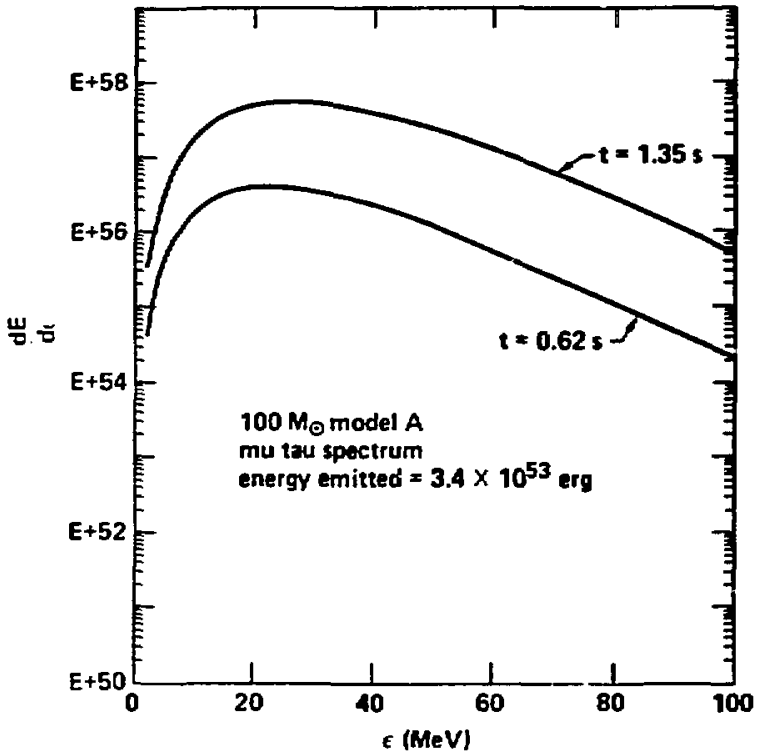

FIGURE 60 


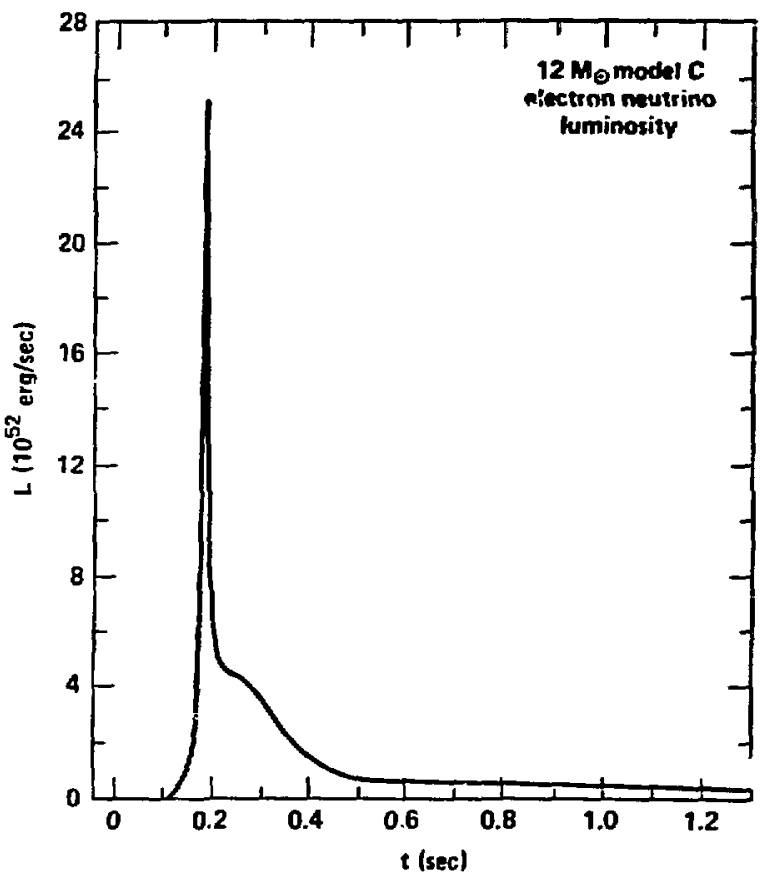

FI GURE 7a 


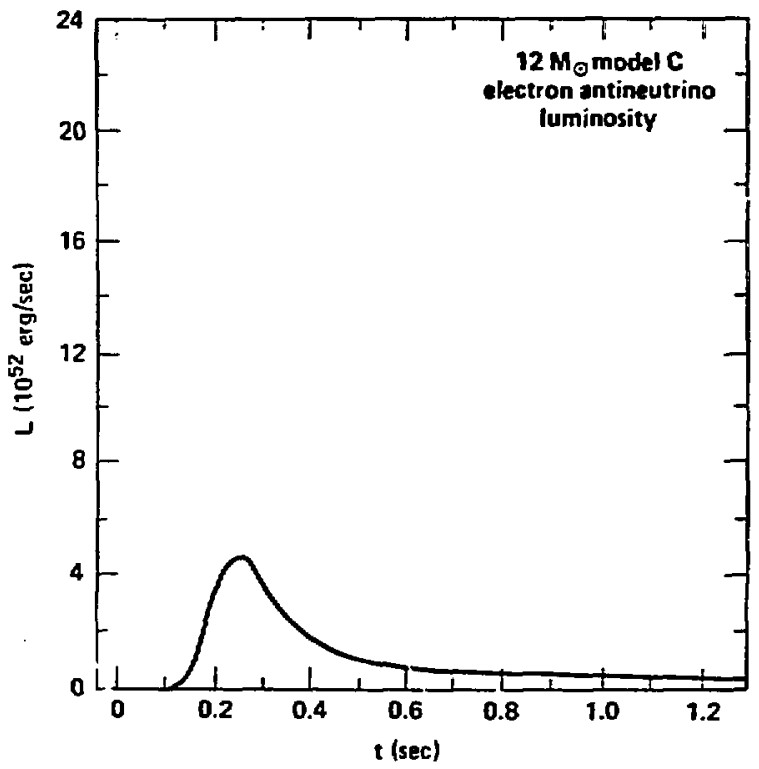

FI GURE Tb 


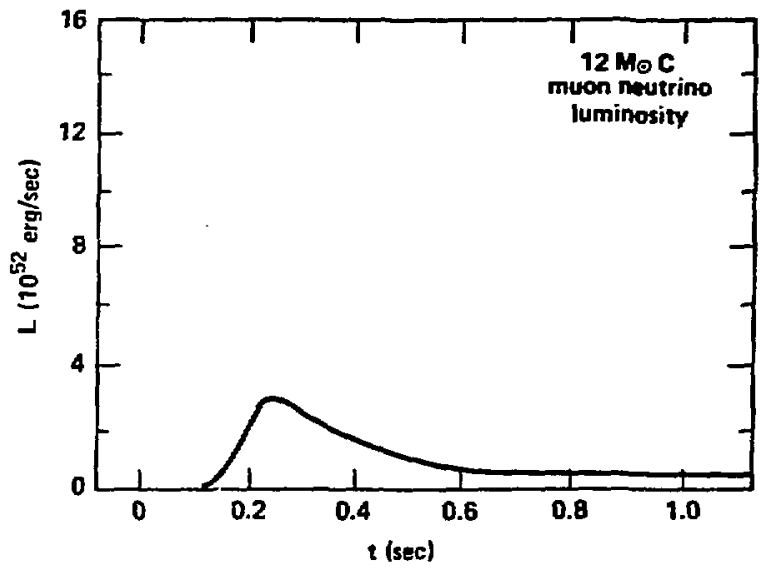

FI GURE 7c 


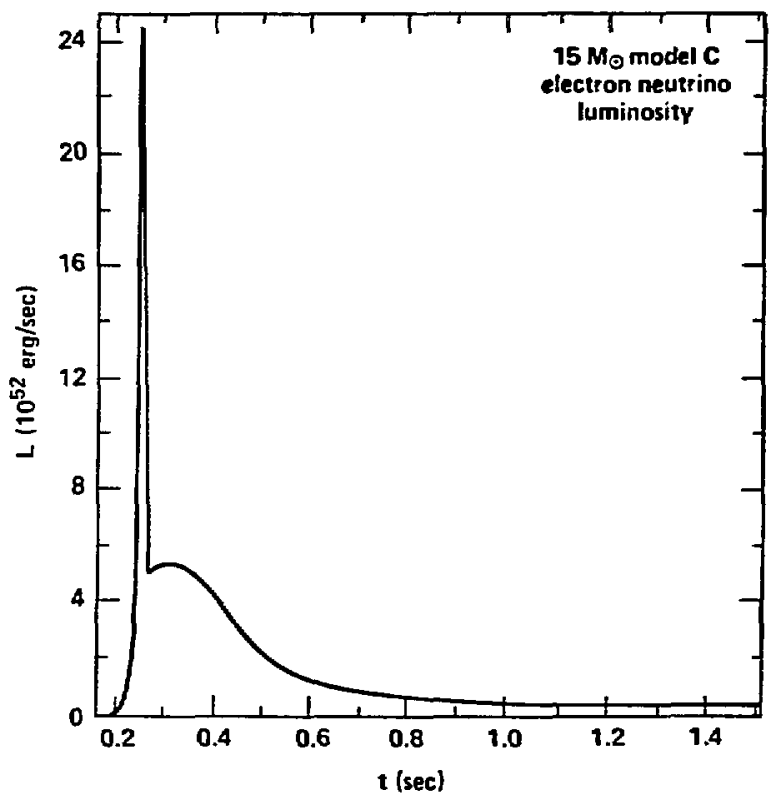

FIGURE $7 \mathrm{à}$ 


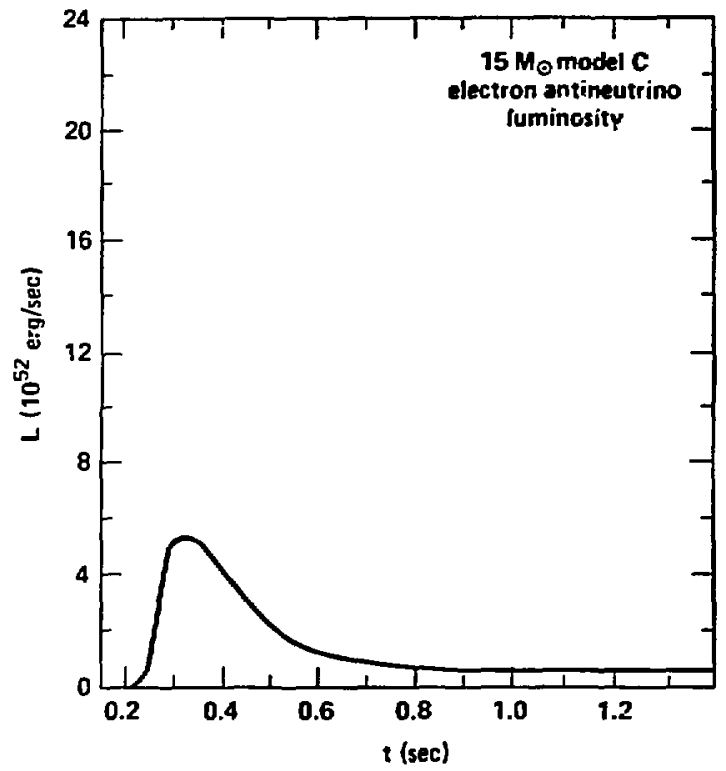

FIGURE 7e 


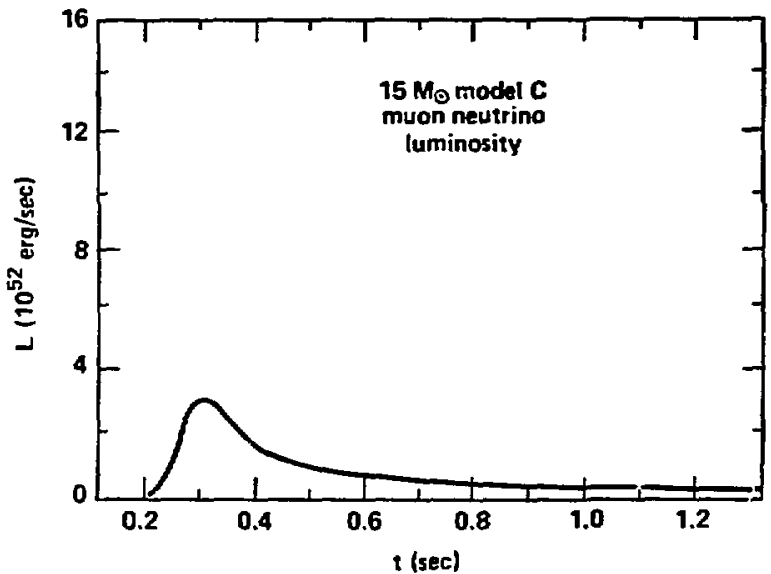

FIGLRE $7 f$ 


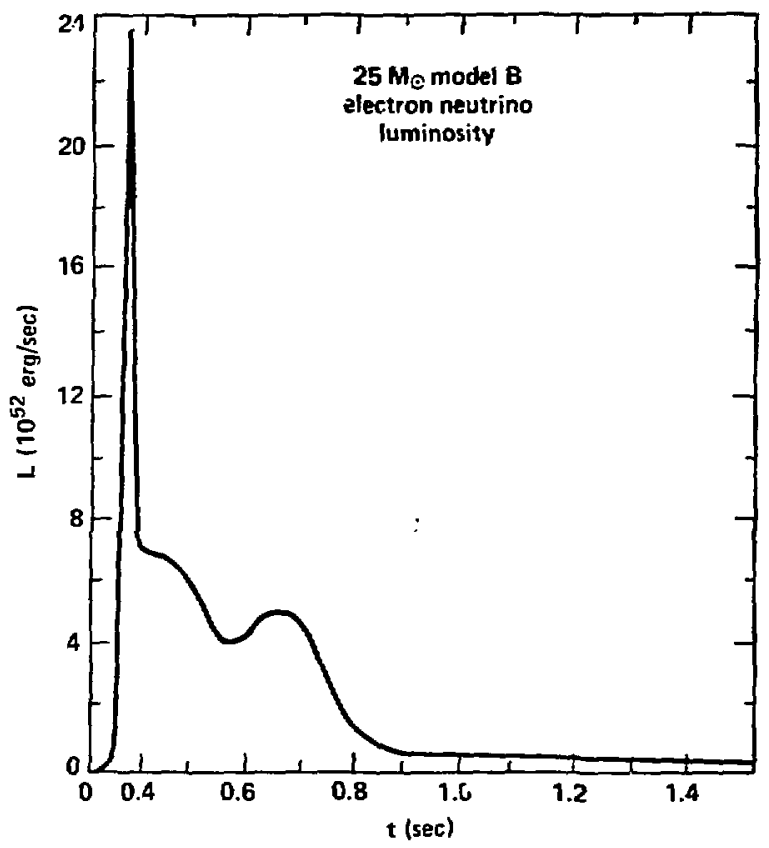

FI GURE $7 E$ 


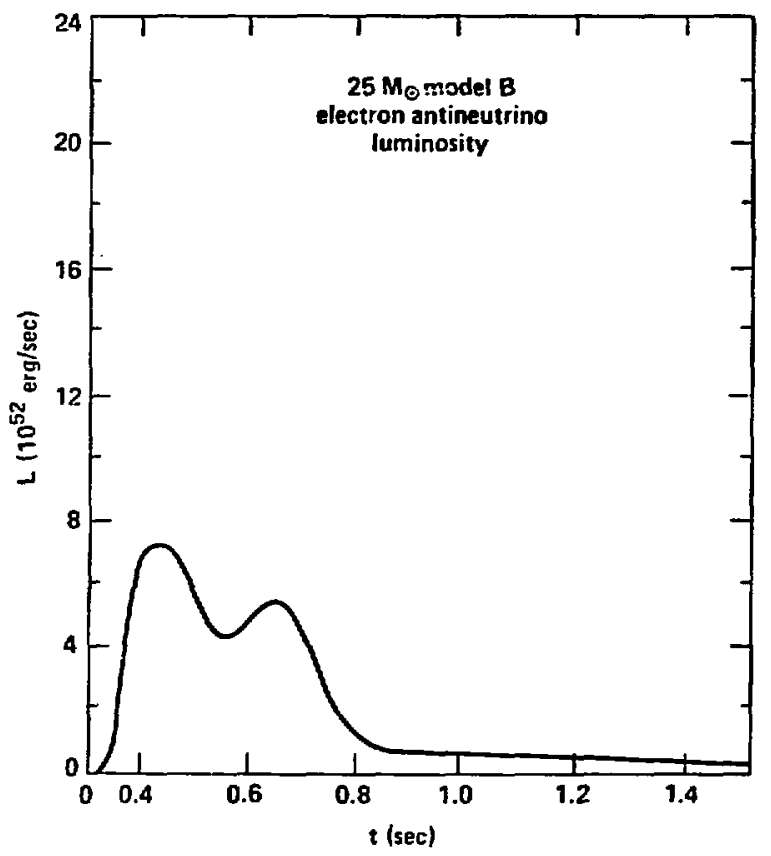

FIGURE 7h 


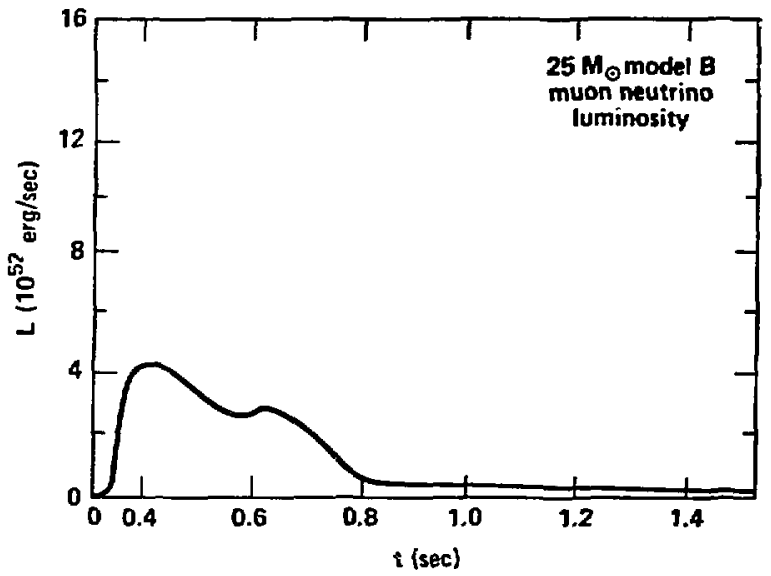

FI GuRe 7 i 


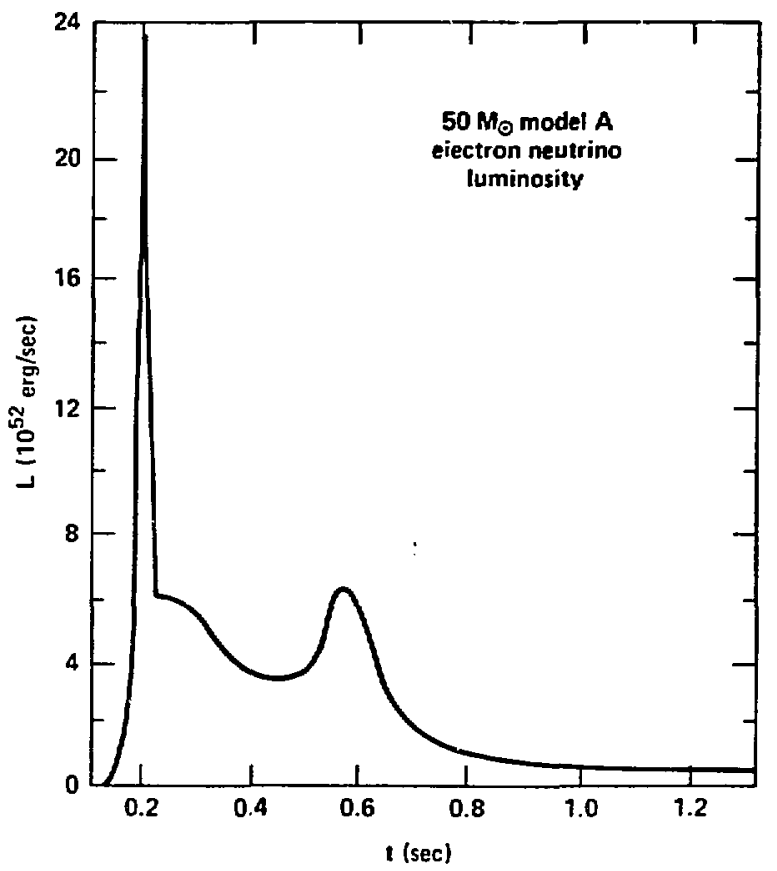

FIGURE 7j 


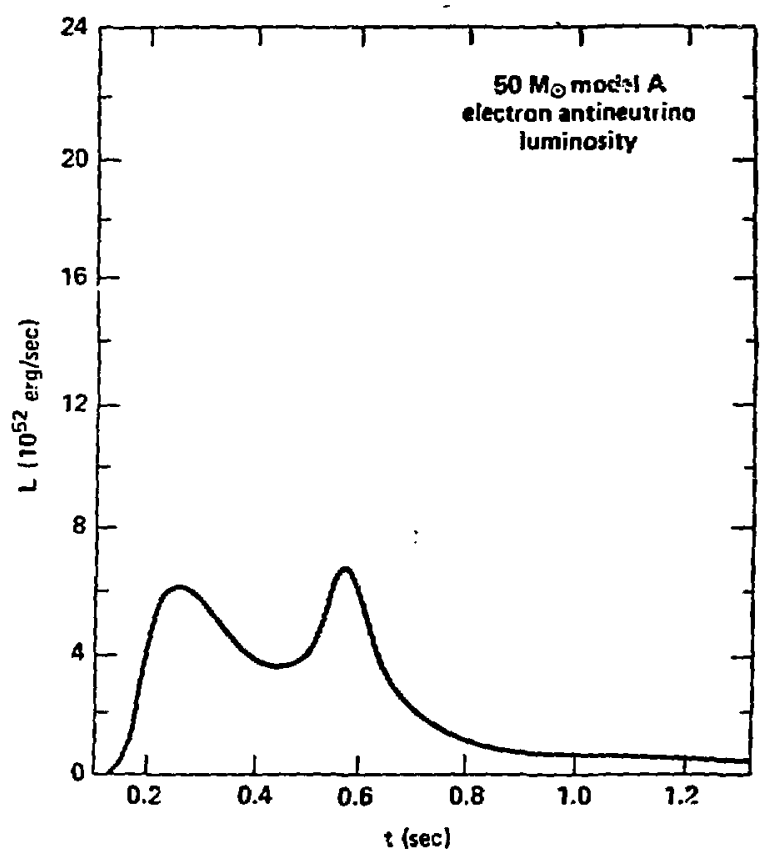

FIGUaz 7k 


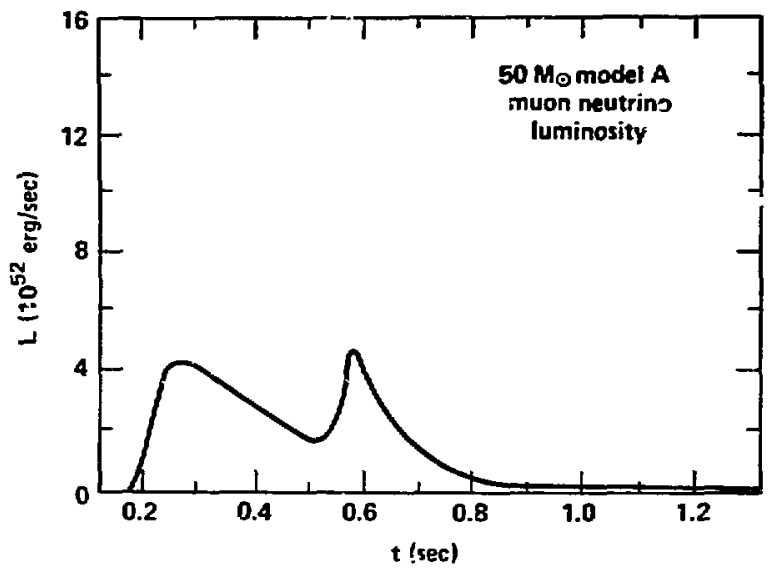

FIGURE 71 
115

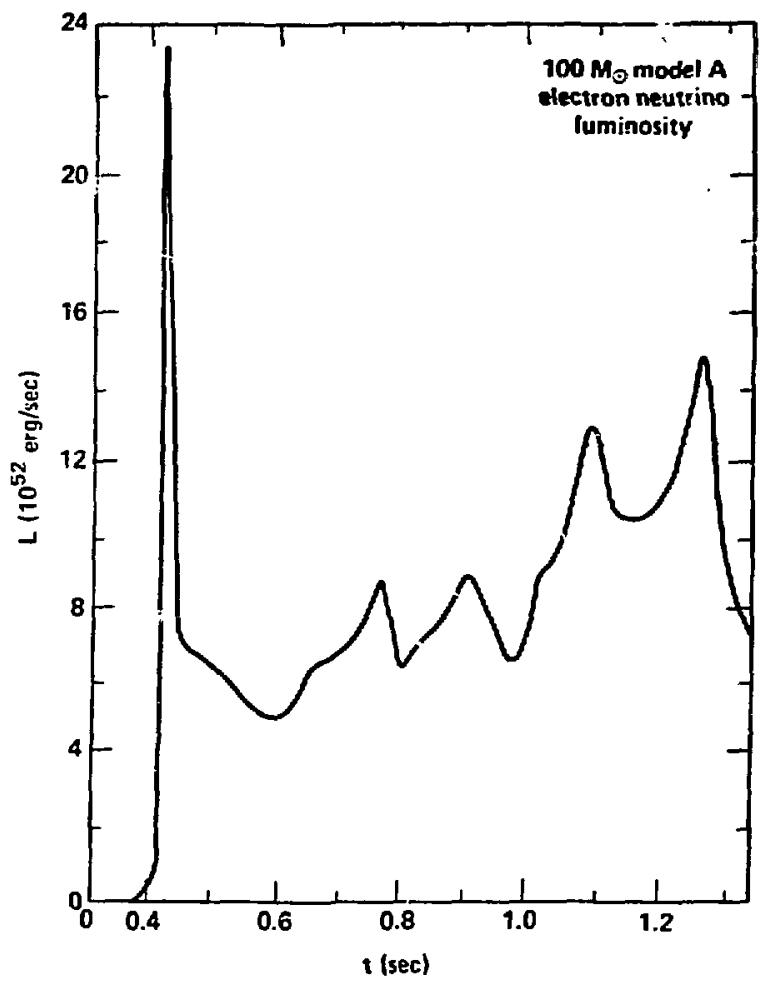

FIOU:ล $7 \mathrm{~m}$ 


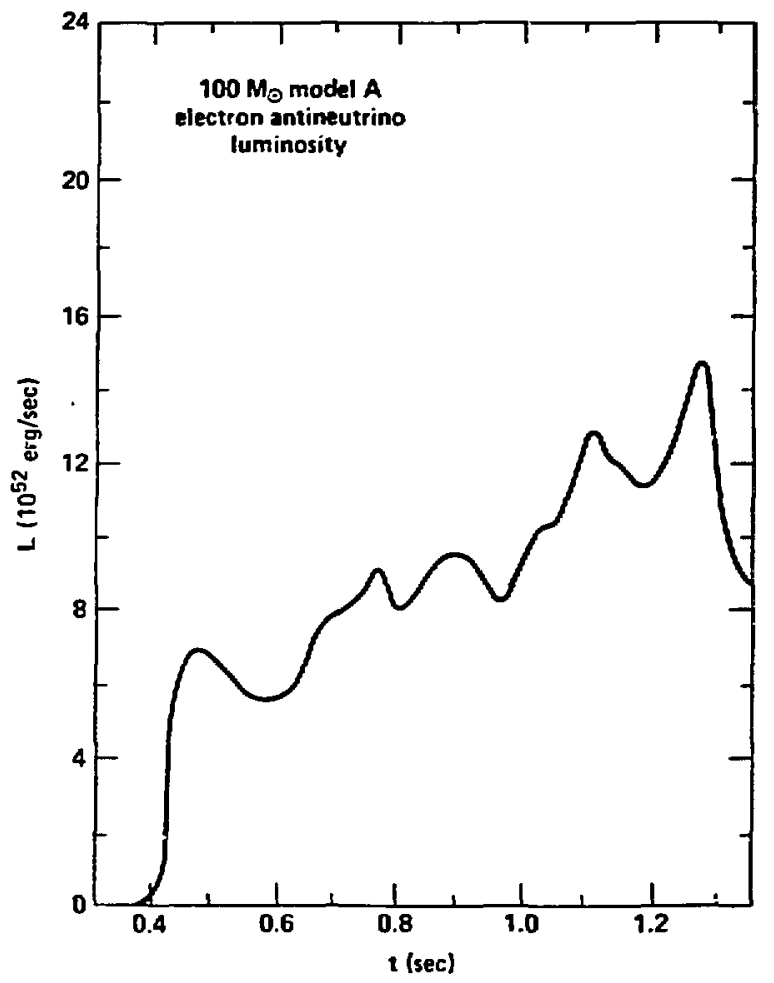

FI GLUE $7 \mathrm{n}$ 


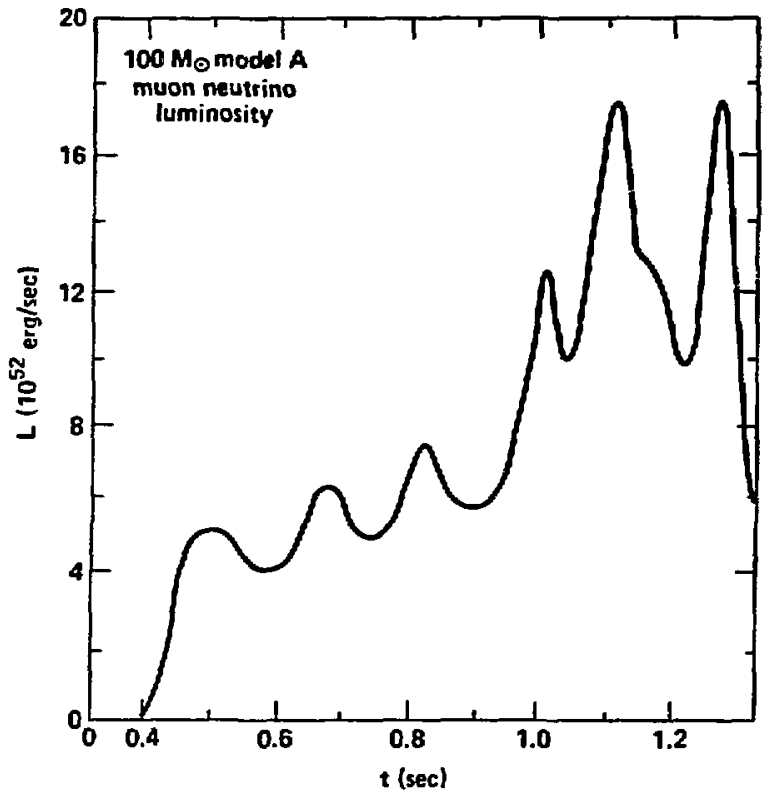

FIGIRE 70 
for number sequence only 


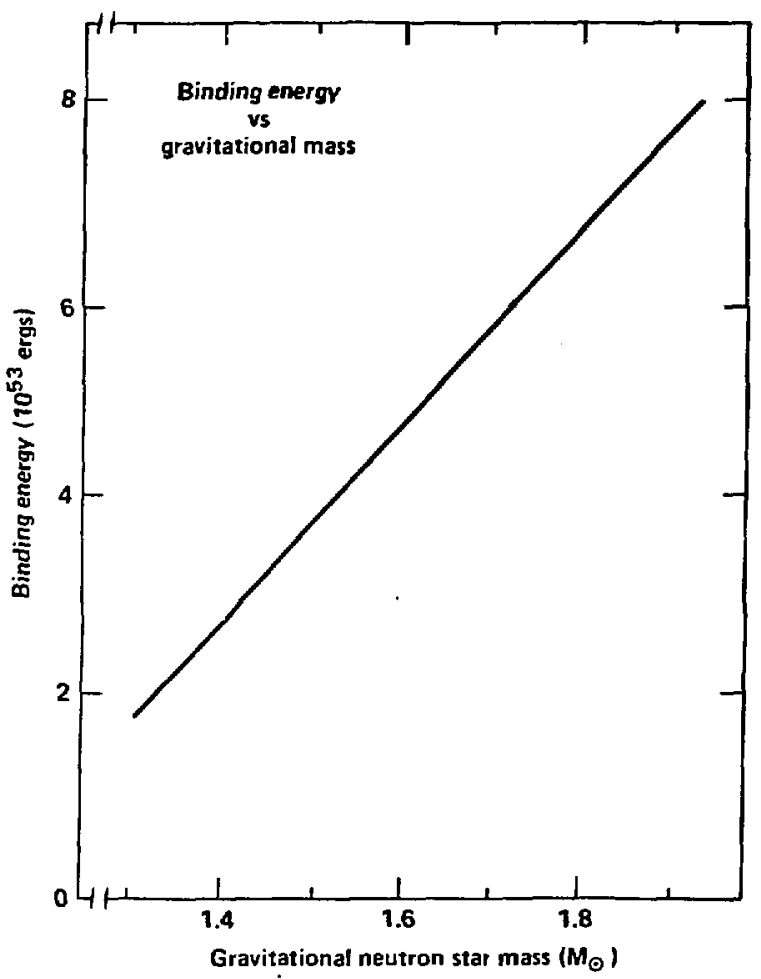

Figues 3 


\section{Chapter V}

\section{CONVECTION IN SUPERNOVA}

\section{INTRODUCTION}

After bounce, and the subsequent leunching of the accretion shock into the outer core, the physical variables, $Y_{Z}$, the lepton number per baryon, and $S$, the entropy per baryon, typically show a dependence on the mas coordinate similar to the one shown in figure 1.

The dip in $Y_{L}$ near the neutrinosphere is due to the following reaction:

$$
p+e^{-} \Longrightarrow n+\nu_{e}
$$

with the neutrino esceping, this part of the core can deleptonize early. The bump in $Y_{L}$ at $.7 M_{\odot}$ is what remains of a discontinuity in $Y_{L}$ produced when the shock began its outward motion.

The current position of the shock is seen as the spike in entropy. It originated around $.7 M_{\odot}$ and, in moving to its present position, has dissociated heavy nuclei into free baryons and helium. Interior to $.7 \%$, the density is so high that a uniform sea of baryons exists. (A profile of the proton fraction is similar in shape to $Y_{L}$.) At earlier times, heavy nuclei exist inside $.7 M_{\odot}$, since the density at the earlier times is not yet high enough to squeeze the nuclei into a uniform sea of nuclear matter.

$Y_{L}$ increases outside the neutrinosphere and beyond the shock postiton. It reaches a value of about .5 and stays relatively constant at .5 throughout the rest of the star. (The neutrino burst from the electron capture during collapse will appear as a bump in $Y_{L}$ approximately $10^{10} \mathrm{~cm}$ from the star center.) The $Y_{L}$ profile is more smooth than the entropy profile near the shock since heavy nuclei outside the shock can participate in electron capture, although only a few protons in any nuclej can capture due to suclear shell effects. Also true, is that the timescale for electron capture is much longer than the dynamical timescale at the densities near the shock. The heavy nuclei pass through the shock and are dissociated before they can capture appreciably.

Entropy is low in the centrai regions of the star initially, and the inner $7 \mathrm{M}_{\odot}$ homologous core remains unshocked during the evolution. Therefore, entropy remains low inside $7 M_{\odot}$. From 7 to $2 M_{\odot}$, the entropy originally produced by the shock has decreased due to the cooling that results from the loss of neutrinos. This cooling has produced a region from 1.5 $M_{\odot}$ to $2 M_{\odot}$ where entropy decreases with increasing mass. 
The core, in this configuration, in unstable in the wense that a redistribution of the material could result in a configuration with lower total energy.

Mixing can occur as the motion of various size parcele of material from one radial position to another. If a parcel, on moving downward, became more denwe that the average surrounding material, it would continue to move downward, nntil it resches a radius where its density was no greater than the surrounding* (anuming no convective overahoot).

Macroconvection describes the limit where mont parcels have a size comparable to the dimensions of the system being considered. In this case, numerical study must take into account the non-spherical nature of the problem due to the large parcel aize. Two studies of core overturn have been done (Livio,Buchler, and Colgate (1980), and Smarr, Wilson,Barton, and Bowers (1981)) using two-dimensional computer codes (axial symmetry is assumed). These studies concentrated on the early times in a supernova explosion (1.e. a few milliseconds after bounce). Both studiea found a portion of the core overturned on a timescale of 5-20 ms. In particular, Smarr et al.(1981) found the region from .7 to $1.25 M_{\odot}$ overturning, but not enough neutrinos were released to achieve an explosion.

Microconvection describes the limit where most parcels have a size much smaller than the entire region of instability. This type of convection can be studied within the context of a spherical computer code by means of a mixing length theory. This chapter is concerned with microconvection, and the manner in which it is incosporated in a computer model for a supernova.

\section{CONVECTION}

Stars, in quasi-static equilibrium, can be unstable, in that a parcel of matter, if moved from its position downward (upward) may become more (less) dense than the surrounding matter, and so be driven even further downward (upward). This type of motion is call convection.

A criterion for the onset of convection is thus:

$$
\left.\frac{\partial \rho}{\partial R}\right|_{\text {ditop }}-\left.\frac{\partial \rho}{\partial R}\right|_{\text {atar }} \leq 0
$$

where $\left.\frac{\partial P}{\partial R}\right|_{\text {disg }}$ is the rate of change of density of the parcel with respect to radius, and $\left.\frac{\partial \rho}{\partial R}\right|_{\text {otar }}$ is the star's density gradient.

Different possibilities for convection occur depending on how the parcel is moved. If the parcel is moved adiabatically, but always in pressure and composition equilibrium, the 
Schwar2schlid criterion for the onset of convection is found.

Using the following figure to illustrate the notation, the Schwarzschild criterion can be derived:

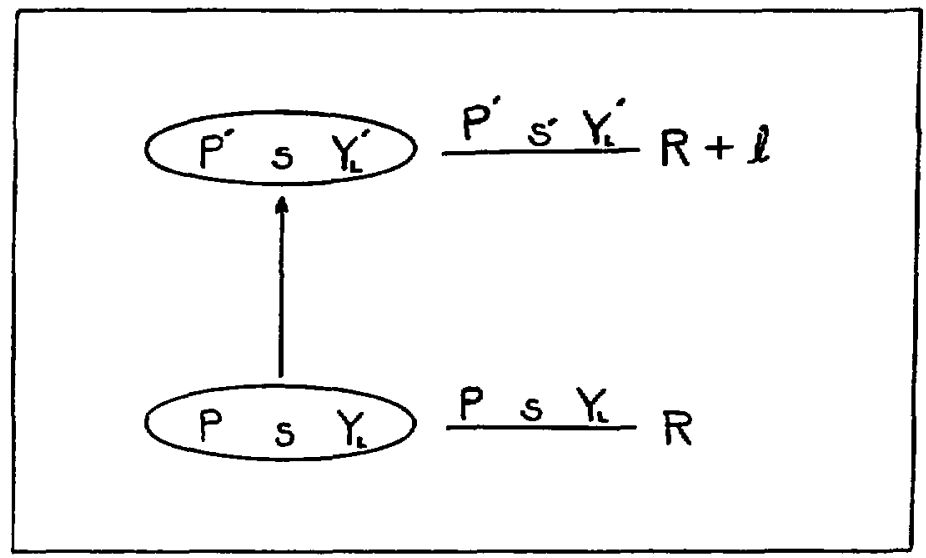

Equation (1) becomes:

$$
\rho\left(P^{\prime}, s^{\prime}, Y_{L}^{\prime}\right)-\rho\left(P^{\prime}, s, Y_{L}^{\prime}\right) \geq 0
$$

Using a Taylor series expansion results in:

$$
\rho\left(P^{\prime}, s^{\prime}, Y_{L}^{\prime}\right)-\rho\left(P^{\prime}, s, Y_{L}^{\prime}\right)=\left(\frac{\partial \rho}{\partial s} \mid P, Y_{L}\right) l \frac{d s}{d R} \geq 0
$$

Now:

$$
\left.\frac{\partial \rho}{\partial s}\right|_{P, Y_{L}}=\frac{T}{C_{P}}\left(\left.\frac{\partial \rho}{\partial T}\right|_{P, Y_{L}}\right) \frac{d s}{d R} \geq 0
$$

For most equations of state $\left.\frac{\partial p}{\partial T}\right|_{P, y_{L}} \leq 0$, and the Schwarzschild criterion becomes:

$$
\frac{d s}{d R} \leq 0
$$


Returning to figure 1 , it is meen that the region from 1.2 to $1.8 \mathrm{M}_{\odot}$ is unatable according to this criterion.

The Ledoux criterion is found by moring the parcel adiabatically, with no componition change but in pressure equilibrium. Then equation (1) becomea:

$$
\rho\left(P^{\prime}, s^{\prime}, Y_{L}^{\prime}\right)-\rho\left(P^{\prime}, \pm, Y_{L}\right) \geq 0
$$

Again using a Taylor series expansion the above equation becomes:

$$
\rho\left(P^{\prime}, s^{\prime}, Y_{L}^{\prime}\right)-\rho\left(P^{\prime}, s, Y_{L}\right)=\left(\left.\frac{\partial \rho}{\partial s}\right|_{P, Y_{L}}\right) l \frac{d s}{d R}+\left(\frac{\partial \rho}{Y_{L}} \mid P, \bullet\right) l \frac{d Y_{L}}{d R} \geq 0
$$

using (2) in (3), the Ledoux criterion becomes:

$$
\left(-\left.\frac{T}{C_{P}} \frac{\partial \rho}{\partial T}\right|_{P, Y_{L}}\right) \frac{d \varepsilon}{d R}+\left(-\left.\frac{\partial \rho}{\partial Y_{L}}\right|_{P, e}\right) \frac{d Y_{L}}{d R} \leq 0
$$

where as before $\left.\frac{\partial P}{\partial T}\right|_{P, Y_{L}} \leq 0$ for most materials. Also $\left.\frac{\partial P}{\partial Y_{L}}\right|_{P_{2},} \leq 0$ since incressing $Y_{Z}$ will result in a density decrease at constant $P$ due to the extrs portion of the preasure now carried by the leptons.

In figure 1 it is seen that the decrease in $Y_{L}$ is very large in the region from the center out to $2 M_{\odot}$. Even though $\frac{d r}{d R} \geq 0$ from the center out to $1.2 M_{\odot}$, the composition gradient is large enough that the region is unstable according to the Ledoux criterion.

Another possibility exists that models the salt finger instability. This instability is discussed in more detail below. For now, move the parcel in temperature and pressure equilibrium, but not composition equilibrium.

The criterion for convection to occur becomes:

$$
\rho\left(P^{\prime}, T^{\prime}, Y_{L}^{\prime}\right)-\rho\left(P^{\prime}, T^{\prime}, Y_{L}\right) \geq 0
$$

or:

$$
\rho\left(P^{\prime}, T^{\prime}, Y_{L}^{\prime}\right)-\rho\left(P^{\prime}, T^{\prime}, Y_{L}\right)=\left(\frac{\partial \rho}{\partial Y_{L}} \mid P, T\right) l \frac{d Y_{L}}{d R} \geq 0
$$

Again $\left.\frac{\partial P}{\partial Y_{L}}\right|_{P, T}$ is generally negative, and the criterion for the salt finger instability to occur is:

$$
\frac{d Y_{2}}{d R} \leq 0
$$


From figure 1 it is meen that the region of the atar form the center to $2 M_{\odot}$ is unatable to ealt fingers.

Convection in collepsed atellar cores that results from the gradient in $Y_{L}$ was frat auggester by Epriein (1979).

MODEL FOR CONVECTION

A standard mixing length theory is used (ree Cox and Giuli (1968)) to model time dependent convection.

A convective velocity is asumed to develop auch that:

$$
\frac{1}{2} \rho v_{C}^{2}=g \delta \rho l
$$

where:

$$
\begin{gathered}
\delta \rho=-\left(\left.\frac{\partial \rho}{\partial R}\right|_{\text {diop }}-\left.\frac{\partial \rho}{\partial R}\right|_{\text {otar }}\right) l \\
g=-\left.\frac{1}{\rho} \frac{d P}{d R}\right|_{\text {oter }}=\frac{P}{\rho l_{P}} \\
l_{P}=-\left.\frac{d R}{d l n P}\right|_{\text {otar }} \\
l=\text { distance parcel moves before mixing }
\end{gathered}
$$

Using:

$$
v_{S}=\left(\frac{\gamma P}{p}\right)^{\frac{1}{2}}
$$

the expression for $v_{C}$ becomes:

$$
v_{C}=\left(\frac{2 l}{\gamma l_{P}}\right)^{\frac{1}{2}}\left(\frac{\delta p}{\rho}\right)^{\frac{1}{2}} v_{S}
$$

Equation (4) is written down by assuming that the change in kinetic energy of a parcel is equal to the work done by the bouyancy force.

The following equations are used as a guide in the construction of sumetical model equations:

$$
\begin{aligned}
& \rho \frac{\partial \bar{Z}}{\partial \tau}=\frac{1}{R^{2}} \frac{\partial}{\partial R}\left(R^{2} \Gamma D \rho \frac{\partial \bar{Z}}{\partial R}\right) \\
& \rho \frac{\partial \epsilon}{\partial \tau}=\frac{1}{R^{2}} \frac{\partial}{\partial R}\left(R^{2} \Gamma D \rho \frac{\partial \epsilon}{\partial R}\right)
\end{aligned}
$$




$$
\rho \frac{\partial Y_{\nu}}{\partial r}=\frac{1}{R^{2}} \frac{\partial}{\partial R}\left(R^{2} \Gamma D \rho \frac{\partial Y_{\nu}}{\partial R}\right)
$$

where:

$$
\begin{gathered}
\bar{Z}=\frac{m_{B}}{\rho}\left(n_{c^{-}}-n_{c^{+}}\right) \\
Y_{\nu}=\frac{m_{B}}{\rho}\left(n_{\nu}\right) \\
\epsilon=\text { matter internal energy/mass } \\
\rho=\text { matter rest mase density } \\
D=\frac{1}{3} v_{C l} l
\end{gathered}
$$

Equation (8) is really 6 equations, one for each neutrino type. The time derivative is a local Eulerian time derivative, and $\Gamma$ is a relativistic correction factor defined in Chapter III and Appendix A.

\section{NUMERTCAL METHOD}

This section describes the method in which convection is introduced into the supernova code. Equations (6), (7), and (8) will used as a guide in the following. Before proceeding further, the assumption that $l=l_{P}$ is made (or that the parcel moves no further than the pressure scale height). Since mixing length theory cannot be expected to give definitive answers but only good guesses as to the effect of the actual physical process, there is some uncertainty in deciding on the choice for $l$, the mixing length.

Once a choice for $l$ is made, a rest mass is defined such that:

$$
\delta m=\frac{\Delta r}{\Delta R} \rho D\left(4 \pi R^{2}\right)=\frac{\Delta r}{\Delta R} \rho\left(4 \times R^{2}\right) \frac{1}{3} v_{C} l
$$

This has the physical interpretation of being the amount of mass that is exchanged between different zones in time $\Delta r$.

Figure 2 illustrates the zoning convention used in the supernova code. Using subscripts $k$, to indicate the zonal position, (9) is rewritten as:

$$
\delta m_{k}=\Delta r \frac{\rho_{k}+\rho_{k+1}}{R_{k+1}-R_{k-1}} 4 \pi R_{k}^{2} \frac{1}{3} v C k l_{k}
$$

Equation (6) then becomes: 


$$
\bar{Z}_{k}^{\prime}-\bar{Z}_{k}=\frac{1}{m_{k}}\left[\Gamma_{k} \delta m_{k}\left(\bar{Z}_{k+1}-\bar{Z}_{k}\right)-\Gamma_{k+1} \delta m_{k+1}\left(\bar{Z}_{k}-\bar{Z}_{k+1}\right)\right]
$$

where $m_{k}$ is the baryon rest mass in zone $k$, and $\vec{Z}_{k}$ is the value of $\bar{Z}_{k}$ at the next time step. Similar equations are written for ( 7$)$ and (8).

The convection equations are colved explicitly (as seen from (10)), in the split operator approximation (i.e. only the convection equations are solved, the other evolution equations are not used. After the new atate variablea are found from the convection equations, the other evolution equations are colved in turn). If more than $25 \%$ of the masa in any zone needs to be mixed in one timestep (typically $10^{-1}$ second), time evolution is halted while the material is moved in a sequence of steps, each of which moves no more than $25 \%$ of the zone mass. After each step, the equation of atate routine is called to determine the theirmodynamic variables.

Since total neutrino number is conserved by (8), but not total neutrino energy, any difference in total neutrino energy found alter the convection aubroutine is called is placed in the matter internal energy. In the region where convection is important, the neutrinos are strongly coupled to the matter. The neutrino fielda will thus adjust to the correct neutrino distribution, and any errors introduced in the distribution function by the convection subroutine should be corrected, as the other subroutines are called in a time step.

Since convection using the Schwarzschild or Ledoux criterion is more traditional an approach than using the salt finger instability, the salt finger instability is discussed in more detail in the following.

\section{SALT FINGERS}

The salt finger mechanism can be illustrated as follows. Imagine a region of hot salty water overlaying a region of cold fresh water. Should a parcel of salt water begin to sink, heat will diffuse from the parcel more quickly than salt (this is just due to the different molecular means of transport of energy by collisions, and transport of salt by particle diffusion). Thus, the salt water can reach temperature equilibrium with the environment sooner than composition equilibrium. However, at the same temperature as fresh water, salt water is more dense due to the salt content, and so will sink further. It will continue to sink in a finger like fashion, producing what is known as a salt finger. 
Now, a similar thought experiment can be done for a mpernova. Imacine a region of neutrons over layer of protons (with electrons to conserve charge). Or course, this is in the presense of a gravitational field with the neutrons in a region of larger gravitational potential. Should a parcel of neutrons begin to sink, collisions with geutrinos of all sypes will allow the parcel to come into temperature equilibrium with the environment mure quickly than composition equilibrium.

The composition changes more slowly since it is determined by the following equation:

$$
\frac{\rho}{m_{B}} \frac{\partial Y_{L}}{\partial r}=\frac{\partial}{\partial \tau} \int\left(F_{\nu_{*}}-F_{\bar{\nu}_{s}}\right) \frac{d E}{E}
$$

while the energy exchange rate is found from:

$$
\frac{\partial e_{m}}{\partial \tau}=\frac{\partial}{\partial \tau} \int\left(F_{\nu_{0}}+F_{\bar{\nu}_{d}}+F_{\nu_{r}}+F_{\bar{\nu}_{p}}+F_{\nu_{r}}+F_{\bar{\nu}_{r}}\right) d E
$$

where $r$ is the local time and $E$ is the local neutrino energy.

Note that mu and tau neutrinos and their antiparticles cannot change composition (for example, $n+\nu_{\mu} \Longleftrightarrow p+\mu^{-}$or $n+\nu_{r} \Longleftrightarrow p+\tau^{-}$) since muoss are too heavy to be produced (i.e. $\frac{m_{\mu} c^{2}}{k_{B} T} \approx 20$ ) in the region in which neutron fingers is important. Tau neutrinos cannot change composition as the mass of a tau particle is $1784 \mathrm{Mev}$ and will not be found in a supernova. The matter and neutrinos are in quasi-equilibrium and the difference in the electron neutrino and antineutrino fluxes determine the nuclear composition. This difference is about $\frac{1}{10}$ the total neutrino flux in all 6 neutrino types which exchange energy with the nuclear matter by scattering as wel) so by emission absorbtion reactions (i.e. $n+\nu_{c} \Longleftrightarrow p+e^{-}$or $\left.p+\bar{\nu}_{e} \Longleftrightarrow n+e^{+}\right)$.

Once the neutron parcel is in temperatinre and pressure equilibrium with the environment, it will be more dense (electron pressure being the difference), as such, the neutron parcel can continue to sink further in a finger like fashion.

The analogy with the salt finger case will be carried further. Linear stability analyses of the salt linger instability (and other doubly diffusive phenomena) aave been carried out by many authors (see Baines and Gill (1969) for a good summary). For the salt finger case, the waveiength of the most unstable mode is given by (see Turner (1971)):

$$
t_{F}=\left(\frac{\kappa_{T} \eta d}{g \alpha \Delta T}\right)^{\frac{1}{d}}
$$

where: 


$$
\begin{gathered}
g=\text { gravitalional act ieration } \\
\alpha=-\frac{1}{\partial T} \frac{\partial \rho}{\partial T} \mid p_{\text {comp }} \\
d=\text { height of region } \\
\Delta T=\text { temperature difference acrose } d \\
x_{T}=\text { thermometric conduetivity } \\
\eta=\text { kinematic viscosity }
\end{gathered}
$$

$l_{F}$ car be taken as an eatimate of the finger width.

Simple kinetic theory arguments can be uned to find:

$$
\kappa_{T}=\frac{1}{3} \lambda_{\nu}=
$$

anc:

$$
\eta=\frac{1}{3} \lambda_{\nu} c \frac{p_{\nu}}{\rho}
$$

In (13) and (14) the following notrtion is used:

$$
\begin{aligned}
& \lambda_{\nu}=\text { neutrino mean free path } \\
& c^{2} p_{\nu}=\text { neutrino energy density }
\end{aligned}
$$

and $\frac{\lambda_{w}}{\hat{R}_{o p}} \leq 1$ (where $R_{a p}$ is the neutrinosphere radius) is being assumed.

Figure 3 shows the inner $2 M_{\odot}$ of a supernova core at late time on a $(\rho, T)$ plane. Most of this region has $\bar{Z}$, the number of matter electrons per baryon, approximately equal to .15 , as convection has been included in the evolution. Without convection $\bar{Z} \approx 33$ would be a more likely value in the central core. Nuclear density has been reached at about $10^{14} \frac{i}{c c}$, at which time nuclear forcss come into play, and the temperature stops rising as pressure suppurt from the replusive nuclear force begins to become important. At $\rho \approx 3.2 \times 10^{14} \frac{\ell}{e c}$, the major preisure oupport is essentially temperature independent, and the temperature lalls as the density increases. Contours of constant $\mu_{e} / k_{B} T$ and $\mu_{\nu_{e}} / k_{B} T$ are shown. Over most of the ster $\mu_{\nu_{c}} / k_{B} T \approx 3$ (or $\mu_{c} / k_{B} T \approx 4$ ) is an indicator of the star track. $\mu_{c}$ and $\mu_{\nu_{c}}$ are related by: 


$$
\mu_{e}-\mu_{v_{0}}=\mu_{N}-\mu_{P}
$$

$\mu_{\nu,}$ and $\mu_{\varepsilon}$ differ more and more $a$ the density increanes owing to the inceming difference in $\mu_{N}$ and $\mu_{P}$. The baryons are well deacribed $a x$ ideal gas outside $.25 M_{O}$ and in this region an eatimate of $I_{5}$ can be made.

The energy of a relativistic lepton-antilepton ga can be written as (see Bludmann and Van Riper (1977)):

$$
E_{l}=\frac{7}{8} a T^{4}\left(1+\frac{30}{7}\left(\frac{\mu_{l}}{\pi k_{B} T}\right)^{2}+\frac{15}{7}\left(\frac{\mu}{\pi k_{B} T}\right)^{4}\right)
$$

where only one spin state has been anumed. For a relativistic electron-positron gas the factor $\frac{7}{8}$ should be replaced by $\frac{7}{4}$.

Using (15) and the ideal gas expremion for energy denaity of a free buryon Eas, the condition:

$$
\rho=\left(1.7 \times 10^{\circ}\right) T_{M}^{3}
$$

can be shown to imply that the neutrinos carry approximately $20 \%$ of the total internal energy. This track in the $(p, T)$ plane also falls close to the atar track outoide $.95 \mathrm{MO}_{\odot}$.

For estimating $I_{F}$, use $\alpha=-\left.\frac{1}{i} \frac{f}{f f}\right|_{P, \text { eomp }}=\frac{1}{3}$, the ideal gas result. Replace $\frac{f}{d}$ with $\frac{G M}{R^{3}}=\frac{1}{r_{\text {tib }}^{2}}$, and equation (12) becomes:

$$
I_{F}=\left(\frac{\lambda_{\nu} c \tau_{v i k}}{3}\right)^{\frac{1}{2}}\left(\frac{\rho_{\nu}}{\rho} \frac{T}{\Delta T}\right)^{\frac{1}{2}}
$$

Now:

$$
\frac{\rho_{\nu}}{\rho}=\frac{E_{\nu}}{\rho c^{2}}=\left(3 \times 10^{-4}\right) T_{M e v} \approx .01
$$

where $T=30 \mathrm{Mev}$ has been taken. Also take:

$$
\frac{\Delta T}{T}=1
$$

and:

$$
T_{\text {vib }} \approx \frac{1}{2} \times 10^{-s} \text { sec. }
$$

to find: 


$$
I_{F}=\left(1 \times 10^{2}\right) \lambda^{1} \mathrm{~cm}
$$

In order to extimute the time for a finger of cise if to come into kemperature cquilibrium with the surroundinge, consider frure 4. Solve:

$$
\frac{\partial E}{\partial t}+\nabla \cdot G_{\nu}=0
$$

inside the cylinder, with the curfece bold at a contant temparature, where $\boldsymbol{E}$ in the total internal enercy dennity, and $G_{v}$ in the wutrino lux. Anome the difinion approximation, and use (15) along with the fect thet $\mu_{y_{1}} / k_{1}, T \sim$ contant to find:

$$
G_{v}=-\frac{4}{3} \lambda_{v} c \frac{\sum_{v}}{T} \frac{\partial T}{\partial r}
$$

In (18), aince the baryons make up at beat $60 \times$ of the totel encroy dencity, ves:

$$
\frac{\partial E}{\partial t} \approx \frac{\Sigma}{T} \frac{\partial T}{\partial t}
$$

and write:

$$
\frac{E}{T} \frac{\partial T}{\partial t}=\frac{1}{r} \frac{\partial}{\partial r}\left(r \frac{4}{3} \lambda_{\nu} c \frac{E_{\nu}}{T} \frac{\partial T}{\partial r}\right)
$$

Since only an entimate in needed, replece (20) with:

$$
\frac{\partial T}{\partial t}=\frac{4}{3} \lambda_{\nu} i \frac{E_{\nu}}{E} \frac{1}{r} \frac{\partial}{\partial r}\left(r \frac{\partial T}{\partial r}\right)
$$

Equation (21) is a diffusion equation with the difusion coefficient:

$$
D=\frac{1}{3} \lambda_{\nu} e \frac{D_{\nu}}{\Sigma}
$$

The time for the cylinder to come into equilibrium is about:

$$
t_{\text {if }}=\left(\frac{\frac{f}{f}}{2.4}\right)^{2} \frac{1}{D}
$$

where 2.4 is the fint sero of the Benel function $J_{0}$. Uning $(17)$ in (22), a value of $t_{2 i}$ is found:

$$
t_{\text {dif }}=1.2 \times 10^{-5} \text { see. }
$$


From the computer model of ealt finger convection, convective velocitive are found to be about $3 \times 10^{3} \mathrm{and}$ and the mixing length (premure acale beight) is about $3 \times 10^{5} \mathrm{~cm}$, implying a lifetime of a ealt finger before mixing to be:

$$
t_{\text {tife }}=10^{-3} \text { sec. }
$$

Therefore, the isothermal model for parcel interchange is a good one to use for walt fingert. Figure 5 shows $l_{F}$ and $l_{P}$ for the ande core shown in figure 3 and at the anme time.

More on convection will tr found in Chepter VII. 


\section{Chapter V Reference}

Baines, P.G. enó Gill, R.E. 1969, J. Fluid Mech., v37, 269.

Rludman, S.A. and Van Riper, K.A. 198\%, Ap. J. v212, 859.

Cox, J.P.and Giuli, R.T. 1968, "Principles of Stellar Structure, Vola, I. and II. (Gordon and Breach: New York) Chapter 14.

Epstein, R.I. 1979, Mon. Not. Roy. Astron. Soc., v188. ,305.

Livio, M.J.R, Buchler,R.J., and Colgate, S.A. 1980, Ap. J. Lettr., v238, L139.

Particle Properties Data 1984, Rev. of Mod. Phys., v56.

Smarr, L., Wilson, J.R., Barton, R.T., and Bowers, R.L. 1981, Ap. J., v246, 515.

Turner, J.S. 1974, Ann. Rrv. Fluid Mech. v6, 37. 


\section{Chapter V Figure Caption:}

Fig. 1 The lepton number per baryon and the entropy per baryon $a$ a function the utar mass.

Fig. 2 The zoning conventions used in the supernove code.

Fig. 3 The track of the central region of a 25 wolar man atar at the late timea on a $(\rho, T)$ plane. Convection was modeled in the evolution of this star.

Fig. $4 \mathrm{~A}$ skematic showing the neutron finger instability.

Fig. 5 The pressure acale height and the vize of a neutron finger a a function of radius. 


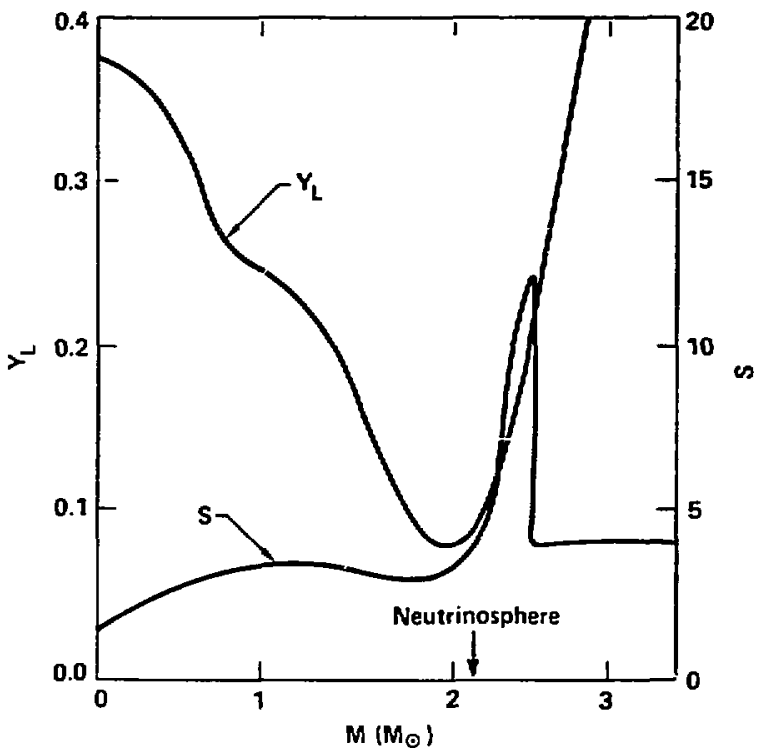

FI GURA 1 


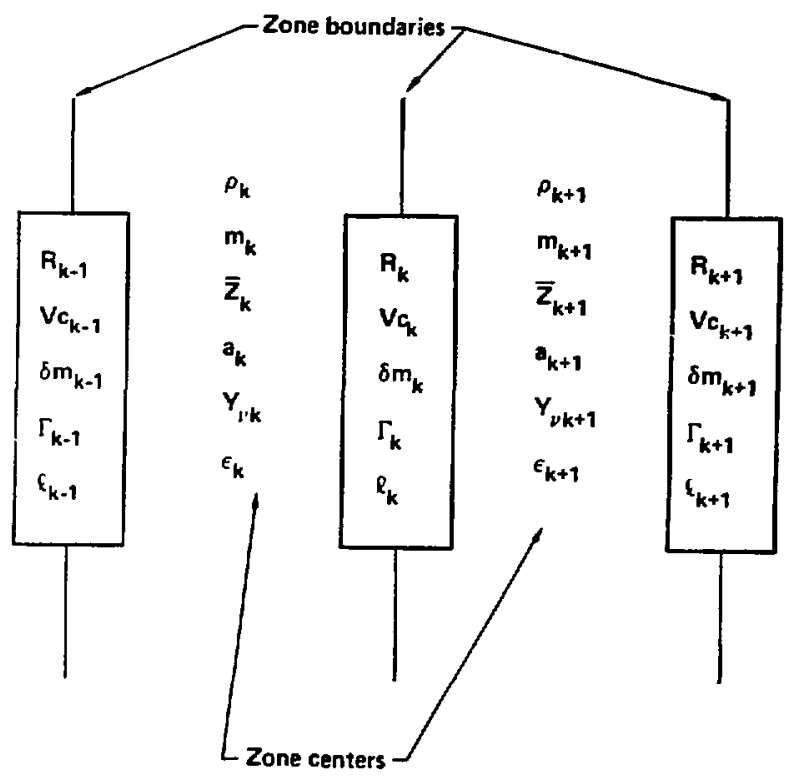

FI GJRE 2 


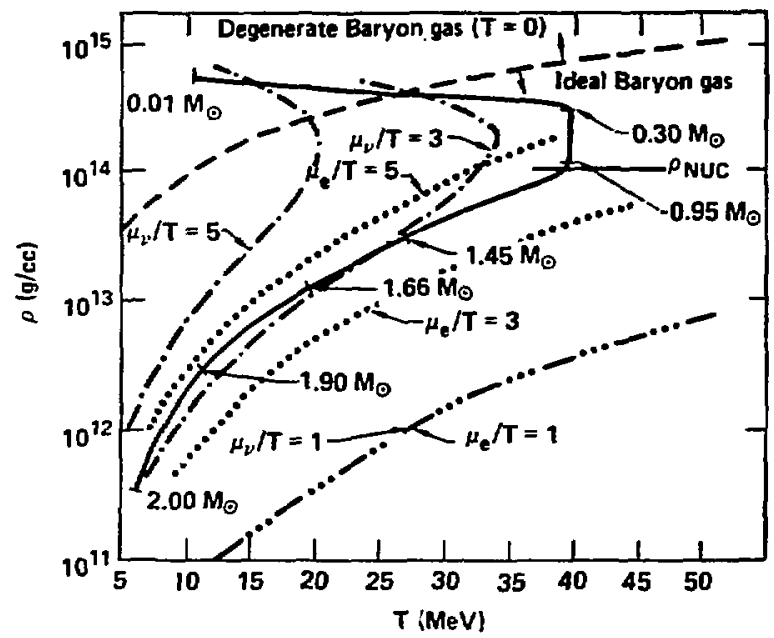

FI GURE 3 


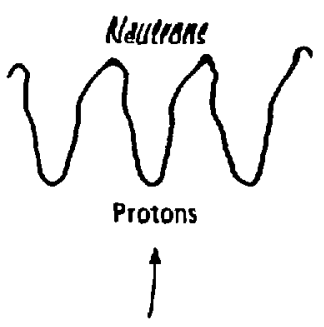

Neutron fingers

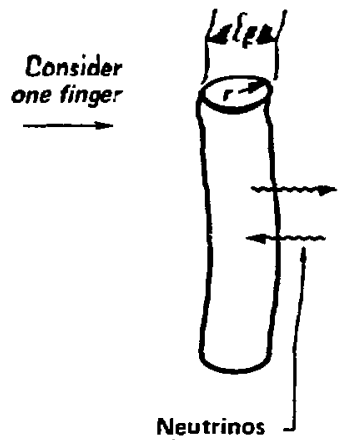

Neutrinos 


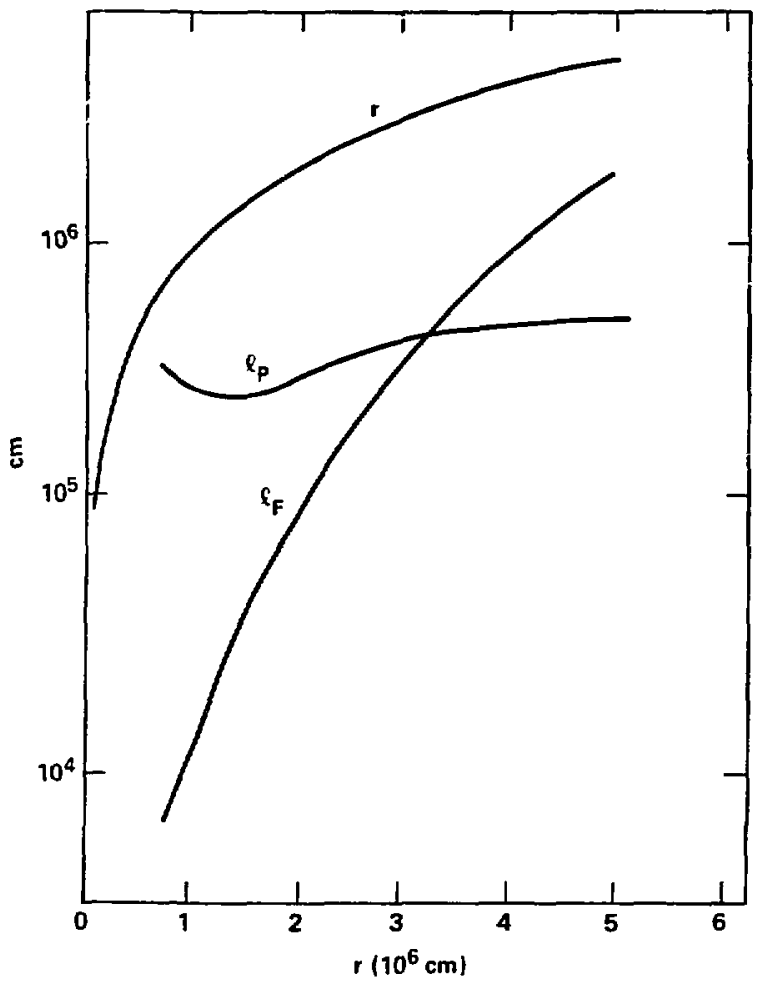

FI GURE 5 


\section{Chapter VI}

\section{RADIATIVE ACCRETION SHOCKS}

\section{INTRODUCTION}

Figure 18 shows the mass point trajectories for $\$ 100 M_{\odot}$ star at late times in a gravitational collapse (see Wilson et a'. (1985)). Figure $1 \mathrm{~b}$ shows the neutrino luminoaity and mav accretion rate for the same star. An oscillatory phenomena is muperimponed over a steady accretion of matter onto the central remnant. To study this oscillation, a aimpler problem can be constructed that models the more complex physics contained in the supernova code.

Going to a simpler problem allows the investigation of the atability of a steady atate solution to small radial perturbations. If the syatem is found to be unatable, then one may be able to understand the late time aupernova in tarms of this inatability. A simpler froblem also allows a smaller number of variables to be used in the defcription of the physics, thereby isolating the effects thought to be most important.

The simpler problem snust contain an oacillatory shock, and a non-degenerate gns of neutrons and protons occupying the region between the shock and the neutrinosphere. Possible interaction of neutrinos and the matter behind the shock must be allowed, along with the possibility of neutrinos being produced by the matter as it reaches the neutrinosphere, due to the impact of the matter onto the remnant. A simple beating-cooling function can model the interaction of neutrinos with the matter, and a boundary condition at the neutrinosphere can replace the surface of the remnant. Strong shock boundary conditions can be used at the shock position.

The physics chosen for the simpler problem is motivated by the present understanding of the oscillations. A lump of matter, as it is stopped by the central remnant, produces neutrinos. Kinetic energy of motion is transformed into thermal energy, which then finds its way into neutrino energy as the beated matter radiates neutrinos. The neutrinos already exiting the neutrinosphere, which originate from within the central remnant, are joined by this accretion burst of neutrinos and increase the heating rate of the neutrons and protons behind the shock. This increases the material pressure behind the shock and pushes the shock wave outwards. The outward movement of the shock reduces the mass accretion rate and reduces the amount of neutrinos produced by accretion. This allows the matter pressure to drop behind the shock, since the heating rate has fallen, and thus the shock begins to 
move inward again, allowing the mass accrevion rate ro increase. The cycle then can repeat, starting with another accretion burat of neutrinow. This acenario io rather complex, and the simplified version is atill a difficult problem to handle.

A steady state solution can be found to the nimpler problem, then perturbation methods can be used to test stability. In this chapter, the cesult found in the construction of ateady state solutions for two geometries, planar and apherical in presented (wee figure 2). The perturbation equations will also be given for both the planar case and the spherical case. The numerical solution of these perturbed equations is not done for whis thesis, but will be the topic of a future paper.

COMPLETE SET OF EQUATIONS FOR THE PLANAR CASE

$$
\begin{gathered}
\frac{\partial \rho}{\partial t}+\frac{\partial}{\partial x}(\rho v)=0 \\
\rho\left(\frac{\partial v}{\partial t}+v \frac{\partial v}{\partial x}\right)=-\frac{\partial P}{\partial x}-\rho g \\
\frac{\partial}{\partial t}(\rho e)+\frac{\partial}{\partial x}(\rho e v)+P \frac{\partial v}{\partial x}=\Lambda_{B C} \\
P=\frac{\rho k_{B} T}{\mu} \\
\frac{P}{\rho e}=\gamma-1 \\
\Lambda_{H C}=\frac{\sigma}{m_{B}} \rho\left(\frac{k_{B}}{m_{e} c^{2}}\right)^{2} \sigma_{\bar{E}}\left(\tau_{d p}^{d i}-T^{6}\right)
\end{gathered}
$$

In the above $T_{s p}$ is the temperature of the neutrinosphere, $\sigma_{B}$ Stephan-Boltzmann constant, $\sigma$ is a constant with the dimensions of $\mathrm{cm}^{2}, k_{B}$ is the Boltzmann constant and $\mu$ is the mean molecular weight. In (2), g is the gravitational acceleration and is assumed constant, as the mass contained in the region from $0 \leq x \leq x_{S}$ (see figure 2) is assumed amall compared to the mass of the compact object. $\Lambda_{H C}$ is constructed to model equation (34) of Chapter II.

BOUNDARY CONDITIONS

At the shock, strong shock boundary conditions are used, and are as follows: 


$$
\begin{aligned}
& \left.P\right|_{z=x_{0}}=\frac{2}{\gamma+1} \rho_{a} u^{2} \\
& \left.v\right|_{z=x_{0}}=-\frac{\gamma-1}{\gamma+1}|u| \\
& \left.\rho\right|_{z=x_{0}}=\frac{\gamma+1}{\gamma-1} \rho_{c}
\end{aligned}
$$

where $u$ is the infall velocity into the shock front, and $p_{a}$ is the ambient density of the matter falling into the shock.

At the neutrinosphere, the following boundary condition is used:

$$
\frac{7}{8} \sigma_{B} T_{o p}^{4}=\frac{1}{2} \rho v^{2} c+F_{C}
$$

where the factor of $\frac{7}{8}$ is due to considering equal contributions from electron neutrinos and antineutrinos ( $\frac{7}{16}$ for each separately, due to Fermi-Dirac statistics rather than BoseEinstein). Equation (9) implies that the kinetic energy of the matter is transformed into blackbody neutrino emission after reaching the neutrinosphere. $F_{C}$ is a core flux, taken to be independent of the mijerial outside the core. For the steady state case, this condition just determines the numerical value of $F_{C}$ to be used in the perturbed problem. Other boundary conditions could be used, such as:

$$
\frac{7}{8} \sigma_{B} T_{s p}^{4}=\frac{1}{2} \rho v^{2} c+\frac{\gamma}{\gamma-1} \frac{c P}{\rho}+F_{C}
$$

This takes into account the fact that the matter must be brought to rest at the neutrinosphere, and contains internal, as well as kinetic energy, that could be converted to neutrinos. As mentioned above, work on the perturbed problem is still in progress, and other boundary conditions must still be tried at $x=0$, in order to test the sensitivity of the results to this inner boundary condition.

\section{STEADY STATE SOLUTION OF THE PLANAR PROBLEM}

In the case where time derivatives are zero, equations (1), (2) and (3) become:

$$
\begin{gathered}
\frac{d}{d x}(\rho v)=0 \\
\rho v \frac{d v}{d x}=-\frac{d P}{d x}-\rho g \\
v \frac{d P}{d x}+\gamma P \frac{d v}{d x}=(\gamma-1) \Lambda_{H C}
\end{gathered}
$$


where the equation of atats has been used in (3) to obtain (12). Equation (10) is easily solved to find:

$$
\rho v=-\rho_{a}|s|=\text { constant }
$$

Using (11) anc (12) to solve for $\frac{d P}{d x}$ and $\frac{y}{x}$ results in:

$$
\begin{gathered}
\frac{d v}{d x}=\frac{1}{\frac{P P}{m^{2}}-1}\left(\frac{g}{v}+\frac{(\gamma-1) \Lambda_{H C}}{\rho v^{2}}\right) \\
\frac{d P}{a x}=-\frac{1}{\frac{7 P}{m^{2}}-1}\left(\frac{\gamma P g}{v^{2}}+\frac{(\gamma-1) \Lambda_{H C}}{v}\right)
\end{gathered}
$$

Since insight into the possible solutions to (13), (14), and (15) can be found by dividing (15) by (14) to find:

$$
\frac{d P}{d v}=-\frac{\frac{{ }^{P} P}{y^{2}}+\frac{(y-1) \Lambda_{H C}}{v}}{\frac{2}{v}+\frac{(\gamma-1) \Lambda_{H C}}{v^{2}}}
$$

Since neither side has an $x$ dependence, a plot of various solutions to (16) (i.e. $P(v)$ ) can be máe using various p:operties of $\frac{d P}{d v}$. For example, all points $(P, v)$ such that $\frac{d P}{d v}=0$, have a curve that passed horizontally through $(P, v)$. Before constructing the plot, it is useful to introduce the following dimensionlese variables:

$$
\begin{gathered}
\bar{P}=\frac{P}{\rho_{a} u^{2}} \\
\bar{w}=-\frac{v}{|u|} \\
\bar{\rho}=\frac{\rho}{\rho_{a}}=-\frac{|u|}{v} \\
\alpha=\frac{\sigma}{m_{B}} \frac{k_{B}^{2} \sigma_{B}}{g|u|} \frac{T_{s p}^{6}}{\left(m_{\varepsilon} c^{2}\right)^{2}} \\
\beta=\alpha\left(\frac{\mu u^{2}}{k_{B} T_{u p}}\right)^{g}
\end{gathered}
$$

Note that $l=x \frac{l}{u^{2}}$ intrnduces $l$, a dimensionless distance.

Using dimensionless $:$ riablea (16) becomes:

$$
\frac{d \bar{P}}{d \bar{w}}=-\frac{\gamma \bar{P}-(\gamma-1)\left(\alpha-\beta(\bar{P} \bar{w})^{6}\right)}{\bar{w}-(\gamma-1)\left(\alpha-\beta(\bar{P} \bar{w})^{6}\right)}
$$


Figure 3 shows a plot of various solutions to (17). The arrows indicate the direction of increasing $x$.

It is also useful to introduce new dependenti variables, the sound apeed and mach number:

$$
\begin{gathered}
c^{2}=\frac{y P}{p} \\
m^{2}=\frac{v^{2}}{\frac{\chi^{P}}{?}}
\end{gathered}
$$

In terms of dimensionless variables (18) and (19) become:

$$
\begin{gathered}
\bar{c}^{2}=\left(\frac{c}{u}\right)^{2}=\gamma \bar{P} \bar{w} \\
\bar{m}^{2}=m^{2}=\frac{\bar{w}}{\gamma \bar{P}}
\end{gathered}
$$

Using $\bar{c}$ and $\bar{m}$, equation (17) becomes:

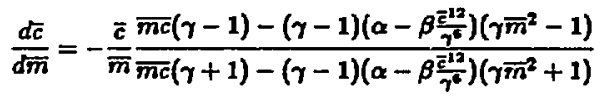

$\bar{c}$ and $\bar{m}$ are useful since there is a linear relationship between $\log \bar{c}, \log \bar{m}$ and $\log \bar{P}$ and $\log \overline{\boldsymbol{w}}$ :

$$
\begin{aligned}
2 \ln \ddot{c} & =\ln \bar{P}+\ln \bar{w}+\ln \gamma \\
2 \ln \bar{m} & =-\ln \bar{P}+\ln \bar{w}-\ln \gamma
\end{aligned}
$$

This is basically a rotation of 45 degrees in thr $\log \bar{P}, \log \bar{w}$ plane.

Figure 3 can be divided into 4 areas, classified as subsonic or supersonic, and net heating greater than zero or net heating less than zero. Along the line $\bar{m}=1, \frac{d \bar{P}}{d d}$ and $\frac{d \bar{W}}{d}$ are both infinite, except for one point, a critical point where both derivatives are finite. The location of this point depends on $\alpha$ and $\beta$, the two dimensionless parameters previouzly defined.

Since the accretion shock is stationary, the infall material velocity must be supersonic and the shocked material velocity subsonic. This restricts attention to the region of figure 3 representing subsonic fiow.

The important fact to be seen in figure 3 is that, given an initial point on a possivie solution curve, and following the curve against the arrows, puts one on a track asymptotic to the net heating equals zero curve. If the post shock temperature is less than the neutrinosphere 
temperature, the initial point in in the net beating $\geq 0$ part of figure 3 . In thin cane, the post shocked material can initially be beated by neutribos, but at the mutter moves clower to $x=0$, and passee over the zero net beating line, it cools and aymptotically approwehes the neutrinouphere temperature. The neutrinowpere tenperature thus acte a a clamp on the matter temperature, not allowing the matter temperature to rise appreciably above the neutrinosphere temperature during infall. If the post ahocked material cemperature is greater than the neutrinosphere temperature, the initial point in fgure $\mathbf{3}$ is in the net heating $\leq \mathbf{0}$ region. In this case the matter temperature monotonically decreases towards the value of the neutrinosphere temperature as the matter moves toward the neutrinosphere, and no beating region forms.

Figures 4a-4g show some results for the numerical integration of (13), (14) and (15), assuming that the post-shock temperature is les than the neutrinosphere temperature. Note figure 4f, a $P$ versus $-v$ diagram, and compare if to figure 3. The neutrinoshere temperature was chosen to be $4 \mathrm{Mev}$, and figure $4 \mathrm{~d}$ showa the matter temperature versus distance, first rising tehind the shock, then asymptotisally approaching $4 \mathrm{Mer}$.

\section{PERTURBATION OF SHOCK-PLANAR CASE-}

The manner in which the perturbation of the time independent wolution is carried out will now be described. A new coordinate, $y$, is used auch that the value of $y$ at the shock position will always have the numerical value $x_{\infty}$, or the unperturbed shocik position. A grid velocity is thus introduced with a wet of grid observers which move so that $y=x_{80}$ at the shock. The relevant equations with \& grid relocity, $v_{G}$, introduced become:

$$
\begin{gathered}
\left.\frac{\partial \rho}{\partial t}\right|_{y}+\left.\frac{\partial}{\partial x}\left(\rho\left(v-v_{G}\right)\right)\right|_{t}+\left.\rho \frac{\partial v_{G}}{\partial x}\right|_{t}=0 \\
\rho\left(\left.\frac{\partial v}{\partial t}\right|_{y}+\left.\left(v-v_{G}\right) \frac{\partial v}{\partial x}\right|_{t}\right)=-\left.\frac{\partial P}{\partial x}\right|_{t}-\rho g \\
\left.\frac{\partial}{\partial t}(\rho e)\right|_{u}+\left.\frac{\partial}{\partial x}\left(\rho e\left(v-v_{G}\right)\right)\right|_{t}+\left.\rho e \frac{\partial v_{G}}{\partial x}\right|_{t}+P \frac{\partial v}{\partial x} l_{t}=\Lambda_{H C}
\end{gathered}
$$

Note that if $v_{G}=0$, Eulerian hydrodynamics is recovered, and if $v_{G}=v$. Lagrangian hydrodynamies is obtained. The shock position is taken ss:

$$
x_{0}=x_{00}+x_{01} e^{w t}
$$




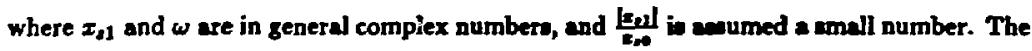
choice for $y$ is:

$$
y=x-x \frac{x_{11}}{x_{20}} e^{w t}
$$

It can be seen that when $x=x_{2}$, the ahock poeition, $y=x_{50}$, to socond order in $\frac{s_{3}}{x_{00}}=\epsilon_{2}$ the smallness parameter used in deriving the perturbation equations.

Introduce the following perturbed variables $P_{1}, p_{1}$, and $v_{2}$ such that:

$$
\begin{aligned}
& P=P_{0}(y)+\epsilon P_{1}(y) e^{\omega t} \\
& \rho=p_{0}(y)+\epsilon P_{1}(y) e^{\omega !} \\
& v=v_{0}(y)+\epsilon v_{1}(y) e^{\omega t}
\end{aligned}
$$

where $P_{0}, p_{0}$, and $v_{0}$ are the unperturbed variables. From (26) the derivatives transform as:

$$
\begin{gathered}
\left.\frac{\partial}{\partial x}\right|_{t}=\left.\left(1-\epsilon e^{\omega t}\right) \frac{\partial}{\partial y}\right|_{t} \\
\left.\frac{\partial}{\partial t}\right|_{x}=\left.\frac{\partial}{\partial t}\right|_{y}-\left.\epsilon \omega y e^{\omega t} \frac{\partial}{\partial y}\right|_{t}=\frac{\partial}{\partial t} l_{L}-\left.v_{G} \frac{\partial}{\partial y}\right|_{t}
\end{gathered}
$$

Identifying the correct grid velocity using (28) results in:

$$
v_{G}=\epsilon \omega y e^{\omega t}
$$

Defining $s$ as wy, the following perturbation equations result when (22), (23) and (24) are expanded using (27) and only terms first order in 6 are retained:

$$
\begin{gathered}
v_{0} \frac{d \rho_{1}}{d y}+\rho_{1}\left(\omega+\frac{d v_{0}}{d y}\right)=-\rho_{0} \frac{d v_{1}}{d y}-v_{1} \frac{d \rho_{0}}{d y}+s \frac{d \rho_{0}}{d y} \\
v_{0} \frac{d v_{1}}{d y}+v_{1}\left(\omega+\frac{d v_{0}}{d y}\right)=-\frac{1}{\rho_{0}}\left(\frac{d P_{1}}{d y}-\frac{\rho_{1}}{\rho_{0}} \frac{d P_{0}}{d y}\right)+s \frac{d v_{0}}{d y}-g \\
v_{0} \frac{d P_{1}}{d y}+P_{1}\left(\omega+\gamma \frac{d v_{0}}{d y}\right)=(\gamma-1)\left(\Lambda_{0}+\Lambda_{1}\right)-v_{1} \frac{d P_{0}}{d y}-\gamma P_{0} \frac{d v_{1}}{d y}+s \frac{d P_{0}}{d y}
\end{gathered}
$$

The neutrinosphere temperature is allowed to oscillate, with the following equation defining $T_{P_{0}}$ and $T_{P_{1}}$ : 


$$
T_{p p}=T_{P_{0}}+e T_{P_{1}} e^{\text {wit }}
$$

The function $\Lambda_{H C}$ is written as:

$$
A_{H C}=\Lambda_{0}+C A_{1} e^{\omega t}
$$

where:

$$
\begin{gathered}
\Lambda_{0}=\frac{\sigma}{m_{B}}\left(\frac{k_{B}}{m_{e} c^{2}}\right)^{2} \sigma_{B} \rho_{0}\left(T_{B}^{6}-T_{0}^{6}\right) \\
\Lambda_{1}=\frac{\sigma}{m_{B}}\left(\frac{k_{B}}{m_{e} c^{2}}\right)^{2} \sigma_{B}\left(6 \rho_{0}\left(T_{P_{1}} T_{P_{Q}}^{5}-T_{1} T_{0}^{5}\right)+\rho_{1}\left(T_{P_{Q}}^{6}-T_{0}^{6}\right)\right)
\end{gathered}
$$

Using $\rho_{0} T_{1}=\frac{\mu}{k_{E}} P_{1}-\rho_{1} T_{0}$ found from $P=\frac{e^{k}, T}{\mu}$, one finda:

$$
\Lambda_{1}=\frac{\sigma}{m_{B}}\left(\frac{k_{B}}{m_{\ell} c^{2}}\right)^{2} \sigma_{B}\left(6 \rho_{0} T_{P_{1}} T_{P_{0}}^{5}-6 T_{0}^{5} \frac{P_{1} \mu}{k_{B}}+p_{1}\left(T_{P_{0}}^{6}+5 T_{0}^{6}\right)\right)
$$

where $T_{0}$ if the unperturbed matter temperature, and $T_{1}$ is the perturbed matter temperature.

Equation (29),(30), and (31) can be written in matrix form as:

$$
A \frac{d D}{d y}+\Omega D+Q D=H
$$

where:

$$
\begin{aligned}
& A=\left(\begin{array}{ccc}
v_{0} & \rho_{0} & 0 \\
0 & v_{0} & \frac{1}{\nu_{0}} \\
0 & \gamma P_{0} & v_{0}
\end{array}\right) \\
& \Omega=\left(\begin{array}{lll}
\omega & 0 & 0 \\
0 & \omega & 0 \\
0 & 0 & \omega
\end{array}\right) \\
& Q=\left(\begin{array}{ccc}
\frac{d v_{0}}{d y} & \frac{d \rho_{Q}}{d y} & 0 \\
-\frac{1}{\rho_{0}^{2}} \frac{d P_{Q}}{d y} & \frac{d v_{0}}{d y} & 0 \\
-(\gamma-1) \frac{\sigma}{m_{B}}\left(\frac{k_{B}}{m_{e} c^{2}}\right)^{2} \sigma_{B}\left(T_{P_{Q}}^{6}+5 T_{0}^{6}\right) & \frac{d P_{0}}{d y} & \gamma \frac{d v_{0}}{d y}+6(\gamma-1) \frac{\sigma}{m_{B}}\left(\frac{k_{D}}{m_{\theta} c^{2}}\right)^{2} \sigma_{B} \frac{\mu}{k_{B}} T_{0}^{5}
\end{array}\right)
\end{aligned}
$$




$$
\begin{aligned}
& D=\left(\begin{array}{l}
\rho_{1} \\
v_{1} \\
P_{1}
\end{array}\right)
\end{aligned}
$$

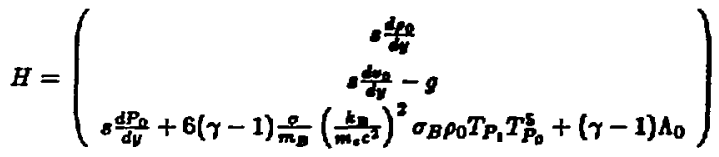

Perturbation of the strong shock boundary conditions (6), (7) and (8) result in:

$$
\begin{gathered}
v_{1 y=x_{\infty 0}}^{\prime}=\frac{2}{\gamma+1} \omega x_{\infty 0} \\
\left.P_{1}\right|_{y=x_{\infty 0}}=\frac{1}{\gamma+1} \rho_{c}|u| \omega x_{\infty 0} \\
\left.\rho_{1}\right|_{y=x_{\infty 0}}=0
\end{gathered}
$$

Perturbation of the inner boundary condition (9) at $x=y=0$ gives the following equation relating $\rho_{1}, v_{1}$, and $T_{P_{1}}$ :

$$
\frac{7}{8} \frac{4 \sigma_{B}}{C} T_{P_{0}}^{3} T_{P_{1}}=\frac{1}{2} \rho_{1} v_{0}^{2}+v_{1} \rho_{0} v_{0}
$$

In solving (32), the value for $A^{-1}$ will be needed and is given by:

$$
A^{-1}=\frac{1}{v_{0}\left(v_{0}^{2}-\frac{\gamma P_{0}}{\rho_{0}}\right)}\left(\begin{array}{ccc}
v_{0}^{2}-\frac{\gamma P_{0}}{\rho_{0}} & -\rho_{0} v_{0} & 1 \\
0 & v_{0}^{2} & -\frac{v_{0}}{\rho_{0}} \\
0 & -v_{0} P_{0} \gamma & v_{0}^{2}
\end{array}\right)
$$

This problem is not really an eigenvalue problem for finding $\omega$, since any value of $\omega$ will yield a possible solution, given the boundary conditions (33) and (34). This occurs since $T_{P_{1}}$, the perturbed neutrinosphere temperature can be adjusted to give a solution, once a value of $\omega$ is chosen.

In preliminary work done on the numerical solution of the perturbed equations, what has become apparent is that $T_{P_{1}}$ can be thought af as a function of $\omega$. The system resonates when the value of $T_{P_{1}}$ becomes a maximum, at those values of $\omega$ that are the natural frequencies of the system. 
COMPLETE SET OF EQUATIONS FOR THE SPHERICAL CASE

$$
\begin{aligned}
& \frac{\partial \rho}{\partial t}+\frac{1}{r^{2}} \frac{\partial}{\partial r}\left(r^{2} \rho v\right)=0 \\
& \frac{\partial v}{\partial t}+v \frac{\partial v}{\partial r}=-\frac{1}{\rho} \frac{\partial P}{\partial r}-g \\
& \frac{\partial}{\partial t}(\rho e)+\frac{1}{r^{2}} \frac{\partial}{\partial r}\left(r^{2} \rho e v\right)+\frac{P}{r^{2}} \frac{\partial}{\partial r}\left(r^{2} v\right)=\Lambda_{H C} \\
& \frac{\partial L}{\partial r}=-4 \pi r^{2} \Lambda_{H C} \\
& g=\frac{G M}{r^{2}} \\
& P=\frac{\rho k_{B} T}{\mu} \\
& \frac{P}{p e}=\gamma-1 \\
& \Lambda_{H C}=\frac{\sigma}{m_{B}} \rho\left(\frac{k_{B} T_{a p}}{m_{e} c^{2}}\right)^{2}\left(\frac{L}{4 \pi r^{2}} f(r)-\frac{7}{8} a c T^{4}\left(\frac{T}{T_{s p}}\right)^{2}\right)
\end{aligned}
$$

The form chosen for $\Lambda_{H C}$ is suggested by equation (34) in Chapter II, but is more general since it allows $L$, the neutrinosphere luminosity, to vary with position (see Bethe and Wilson (1985)).

The factor $f(r)$ in (41) takes into account the fact that integration c.ver the solid angle subtended by the neutrinosphere at position $r$, only approximately done in obtaining (2.34) must be done correctly as $r$ approaches $r_{s p}$, the neutrinosphere radius. However, even if $f(r)=2\left(\frac{r}{r_{a p}}\right)^{2}\left(1-\sqrt{1-\left(\frac{r_{o p}}{r}\right)^{2}}\right)$, the value found when exact solid integration is used, $\Lambda_{H C}$ fails to become zero when $r=r_{\mathrm{sp}}, T=T_{\mathrm{ap}}$, and $L=4 \pi r_{\mathrm{ap}}^{2}\left(\frac{7}{8} \sigma_{B} T_{\mathrm{sp}}^{4}\right)$, as it should. The reason for the discrepancy is due to the approximation of the neutrinosphere as a sharp Burface, with a discontinuous change from an optically thick region to an optically thin one. The real neutrinosphere occupies a finite region in the radial coordinate. A form for $f(r)$ 
is chosen to force $\Lambda_{H C}$ to be zero at the neutrinophere, but not effect the correct large $r$ behavior. The form chosen for $f(r)$ is as follows:

$$
f(r)=\left(1+3\left(\frac{r_{m}}{r}\right)^{2}\right)
$$

The amount of matter in the region $r_{o p} \leq r \leq r$, in asumed amall as compared to the central remnant mass, and $20 \mathrm{~g}$, the gravitational acceleration is given by (39) with $M$ the approximately constant central mass. The only previously undefined constant appearing in (35) to (41) is $a$, which is defined as $\frac{40 n}{c}$.

\section{BOUNDARY CONDITIC NS}

The infalling material is aseumed to be composed of iron, with $8 \mathrm{Mer}$ per nucleon in binding energy. The shock is assumed strong enough to dissociate the iron to free baryons. and the energy associated with this breakup must be included in the shock jump conditions. At the shock, the boundary conditions are:

$$
\begin{gathered}
\left.k_{B} T\right|_{r=r_{\theta}}=\frac{3}{32} m_{B} u^{2}\left(1+\sqrt{\left(1+\frac{32}{9} \frac{\epsilon_{B}}{m_{B} u^{2}}\right)}\right)-\frac{\epsilon_{B}}{2} \\
\left.v\right|_{r=r_{+}}=-|u|\left(\frac{5}{8}-\frac{3}{8} \sqrt{\left(1+\frac{32}{9} \frac{\epsilon_{B}}{m_{B} u^{2}}\right)}\right) \\
\left.\rho\right|_{r=r_{+}}=\frac{\rho_{a}}{\left.\frac{5}{8}-\frac{3}{8} \sqrt{\left(1+\frac{32}{9} \frac{\epsilon_{B}}{m_{B} u^{2}}\right.}\right)}
\end{gathered}
$$

where $\left.P\right|_{r=r}$, can be found using (40). These jump conditions are done for a $\gamma=\frac{5}{3}$ gas only. $\epsilon_{B}$ is $1.28: 10^{-5} \operatorname{erg}(8 \mathrm{Mev}), u$ is the infall velocity into the shock, and $\rho_{a}$ is the ambient density of the infalling material. The choice $|u|=\sqrt{\frac{2 G M}{r \cdot}}$ suggests itself as a free fall velocity to be used at the outer boundary.

A discussion of general time dependent boundary conditions at $r=r$, will not be attempted in this thesis. The boundary conditions for the steady state problem at $r=r_{s p}$ are:

$$
\begin{gathered}
T=T_{o p} \\
L=\frac{7}{8} \sigma_{B} T_{u p}^{4}\left(4 \pi r_{s p}^{2}\right)
\end{gathered}
$$




\section{STEADY STATE SOLUTION OF THE SPHERICAL PROBLEM}

The time derivatives in equatione (35), (36) and (37) are net to sero, and these three equations are manipulated to give:

$$
\begin{gathered}
r^{2} \rho v=\text { constant } \\
\frac{d v}{d r}=\frac{1}{\frac{\gamma^{P}}{v^{2}}-1}\left(\frac{G M}{r^{2} v}+\frac{(\gamma-1) \Lambda_{H C}-2 \gamma \frac{P_{q}}{r}}{\rho v^{2}}\right) \\
\frac{d P}{d r}=-\frac{1}{\frac{T^{P}}{\omega^{2}}-1}\left(\frac{\gamma P G M}{r^{2} v^{2}}+\frac{(\gamma-1) \Lambda_{H C}-2 \gamma \frac{P_{v}}{r}}{v}\right)
\end{gathered}
$$

Using the time independent form of (35), (36) and (37), along with (38) allows the following equation to be constructed:

$$
\frac{1}{r^{2}} \frac{d}{d r}\left(r^{2}\left(\rho v\left(e+\frac{P}{\rho}+\frac{1}{2} v^{2}-\frac{G M}{r}\right)+\frac{L}{4 \pi r^{2}}\right)\right)=0
$$

Equation (50) is a statement of total energy flux conservation, and can be integrated to give:

$$
L=l_{0}+\dot{M}\left(\frac{\gamma}{\gamma-1} \frac{P}{\rho}+\frac{1}{2} v^{2}-\frac{G M}{r}\right)
$$

where $l_{0}$ is a constant, and $\dot{M}=-4 \pi r^{2} \rho v$ is the constant mass accretion rate (see equation (47)).

Results of the numerical integration of the steady atate spherical problem are seen in figures $5 \mathrm{a}$ through $5 \mathrm{~g}$, which show plots similar to figures $\mathbf{4 a}$ through $\mathbf{4 g}$. Figure 5 h shows the luminosity versus radius curve.

From the results of the numerical integration, near the neutrinosphere, the matter temperature, as a function of radius, can be approximated by demanding $\Lambda_{H C} \approx 0$, or:

$$
T=\left(\frac{T_{o p}^{2}}{\frac{7}{8} a c} \frac{L}{4 \pi r^{2}} f(r)\right)^{\frac{1}{2}}
$$

Comparing figures $5 \mathrm{a}$ through $5 \mathrm{~h}$ to the output of the supernova code taken at a late time, but before explosion, shows remarkable agreement with the steady state results found here. Even the decrease, and then increase of the density behind the shock (see figure $5 \mathrm{c}$ ) was reproduced in the supernova code output. 
Figure $5_{\mathbf{g}}$, the curve of $\Lambda_{H C}$ veraus radius whows the matter, after being diseciated can absorb energy from the neutrinos. The matter is much colder than it would have been if dissociation had not occured, vince $8 \mathrm{Mev}$ per nucleon of the infall kinetic energy went into the iron breakup and is thus unavailable for ahock heating. This implies the pressure is low and the material quickly begins to accelerate under the influence of gravity. Since the matter is being compressed as it falls, due at firct to geometric effects, and later to the increasing pressure, it heats up and begins to radiate neutrinos. It woon radiates more energy than it absorbs and a region of cooling exists as the material falls to the neutrinoaphere, now decelerating due to the high pressure gradients.

The temperature of the neutrinosphere thus acts as a clamp on the matter temperature at late times when baryons occupy the region outside the neutrinosphere up to the shock position.

\section{PERTURBATION OF SHOCK-SPHERICAL CASE-.}

A similar proceedure was used to derive perturbation equations for th= opherical case, as for the planar case. The resulting formula appear below:

$$
A \frac{d D}{d R}+\Omega D+Q D=H
$$

where:

$$
\begin{aligned}
& A=\left(\begin{array}{cccc}
v_{0} & \rho_{0} & 0 & 0 \\
0 & v_{0} & \frac{2}{\rho_{0}} & 0 \\
0 & \gamma P_{0} & v_{0} & \frac{\gamma-1}{1 \pi R^{2}} \\
0 & 0 & 0 & \frac{1}{4 \pi R^{2}}
\end{array}\right) \\
& \Omega=\left(\begin{array}{llll}
\omega & 0 & 0 & 0 \\
0 & \omega & 0 & 0 \\
0 & 0 & \omega & 0 \\
0 & 0 & 0 & 0
\end{array}\right) \\
& Q=\left(\begin{array}{cccc}
\frac{d v_{0}}{d R}+2 \frac{v_{0}}{R} & \frac{d \rho_{0}}{d R}+2 \frac{p_{0}}{R} & 0 & 0 \\
-\frac{1}{\rho_{0}^{2}} \frac{d P_{0}}{d R} & \frac{d v_{0}}{d R} & 0 & 0 \\
0 & \frac{d P_{0}}{d R}+2 \frac{2 P_{0}}{R} & \frac{\gamma d v_{0}}{d R}+2 \frac{T v_{0}}{R} & 0 \\
\frac{\mathrm{r}}{\rho_{0}}\left(\frac{L_{0} /(R)}{4 \pi R^{2}}+\frac{36}{8} a c T_{0}^{4}\left(\frac{T_{0}}{T_{P_{0}}}\right)^{2}\right) & 0 & -\frac{\mathrm{r}_{\mu}}{\rho_{0} L}\left(\frac{21}{4} a c T_{0}^{3}\left(\frac{T_{0}}{T_{r_{0}}}\right)^{2}\right) & \frac{\mathrm{r} f(R)}{4 \pi R^{2}}
\end{array}\right)
\end{aligned}
$$




$$
\begin{aligned}
& D=\left(\begin{array}{l}
p_{1} \\
v_{1} \\
P_{1} \\
L_{1}
\end{array}\right)
\end{aligned}
$$

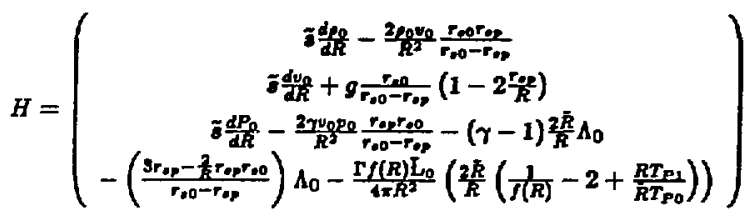

$$
\begin{aligned}
& A^{-1}=\frac{1}{v_{0}^{2}-\frac{\gamma P_{0}}{\rho_{0}}}\left(\begin{array}{cccc}
\frac{v_{0}^{2}-\frac{\gamma P_{0}}{v_{0}}}{v_{0}} & -p_{0} & \frac{1}{v_{0}} & -\frac{\gamma-1}{v_{0}} \\
0 & v_{0} & -\frac{1}{\nu_{0}} & \frac{\gamma-1}{\nu_{0}} \\
0 & -\gamma P_{0} & v_{0} & -(\gamma-1) v_{0} \\
0 & 0 & 0 & \left(v_{0}^{2}-\frac{\gamma P_{0}}{\nu_{0}}\right) 4 \pi R^{2}
\end{array}\right)
\end{aligned}
$$

and new variables appearing in equations (52) through (57) are defined as follows:

$$
\begin{gathered}
\Gamma=\frac{\sigma}{m_{B}}\left(\frac{k_{B} T_{P 0}}{m_{e} c^{2}}\right)^{2} p_{0} \\
\tilde{s}=\left(\frac{R-r_{o p}}{r_{s 0}-r_{o p}}\right) \omega r_{s 0} \\
\tilde{R}=\left(\frac{R-r_{s p}}{r_{s 0}-r_{s p}}\right) r_{s 0} \\
r=R+\left(\frac{R-r_{s p}}{r_{s 0}-r_{o p}}\right) \epsilon r_{s 0} e^{\omega t} \\
L=L_{0}+\epsilon L_{1} e^{\omega t}
\end{gathered}
$$

Note that the variables in equations (52) through (57) are complex in general. Equation (52) is thus a $4 \times 4$ matrix equation and is equivalent to two $4 \times 4$ matrix equations containing real variables. Of course, this is also true in the planar case previously presented where the equation corresponding to (52) is a complex $3 \times 3$ matrix equation equivalent to two $3 \times 3$ real matrix equations. 


\section{BOUNDARY CONDITIONS}

The perturbed boundary conditions are given for the outer boundary only, $R=r_{80}$, and are as follows:

$$
\begin{gathered}
\frac{k_{B} T_{1}}{m_{B}}=\frac{3}{32}|u| \omega r_{\infty 0}\left(2+\varsigma+\frac{1}{\varsigma}\right) \\
v_{1}=\frac{3}{8} \omega r_{\infty 0}\left(1+\frac{1}{\zeta}\right) \\
\rho_{1}=8 \rho_{a} \frac{\omega r_{00}}{|u|}\left(\frac{32}{9 m_{B} u^{2}} \epsilon_{B}\right)\left(\frac{1}{(5-3 \zeta)^{2} \zeta}\right)
\end{gathered}
$$

where $s=\sqrt{\left(1+\frac{92}{m_{B} u^{2}}\right)}$.

\section{SUMMARY AND SPECULATIONS}

From a study of the steady state solution to the problem described in the introduction, it has been found that in the region between the neutrinosphere and the shock, the matter temperature cannot rise above the neutrinosphere temperature as the matter falls onto the remnant, if the post-shock temperature is less than the neutrinosphere temperature. If the post-shock temperature is higher than the neutrinosphere temperature, the temperature of the matter monotonically decreases to the neutrinosphere temperature as the material moves to the central remanant. This occurs because the matter can cool by neutrino emission if the temperatures try to rise above the neutrinosphere temperature. Therefore, for a heating region to form, the post-shock temperature must be less than the neutrinosphere temperature. In the spherical case, this is almost guaranteed to occur, since the dissaciation of heavy elements robs infall kinetic energy that could otherwise go into shock heating. In the planar case considered, it was easy to find solutions where the post shock temperature was higher than the neutrinosphere temperature and no heating region formed. No dissociation was assumed to take place for the planar case. Of course, it is also possible to force no heating behind the shock in the epherical case, but this requires the infall velocity to be much greater than $\sqrt{\frac{2 G M}{r_{0}}}$ and it is not easy to see 8 way to avoid $|u| \approx \sqrt{\frac{2 G M}{r_{0}}}$ being the case.

I wish to speculate on the possible future results of a perturbation analysis using the steady state solution. Looking at figure $5 \mathrm{~g}$, it seems likely that a perturbation at a frequency that produces a $\Lambda_{1}$ (the perturbed heating-cooling function) with a spatial "wavelength" 
that fits one wavelength between the neutrinosphere and the ahock wili produce the fund:mental mode of cscillation seen in the computer simulation. If this in the case, it implies the ascillation in neutrino luminosity is not produced by material near the surface of the core, but by all the material in the region between the neutrinosphere and the shock. I hope this is the case, as it implies the solutions to the perturbation equations may not be overly sensitive to the boundary conditions at the neutrinosphere. 


\section{Chapter VI References}

Wilson, J.R., Mayle, R., Woosley, S.E, and Weaver, T.A. 1985, XII Texas Symp. Rel. Ap. , in press. 


\section{Chapter VI Figure Captions}

Fig. 1 Mas point trajectories for a 100 solar mass atar undergoing gravitational collapee. The luminosity and mass accretion rate are shown in the accompanying figure.

Fig. 2 Skematic drawing of the simplified problem of a radiative shock in the presence of neutrino exiting the neutrinosphere.

Fig. 3 Pressure versus velocity solutions for the planar problem.

Fig. 4 Results of numerical integration of the oteady state equation of motion for the planar problern ( $\mathrm{r}$ of $\frac{1}{\mathrm{~s}}$ was used).

Fig. 5 Results of numerical integration of the steady state equations of motion for the spherical problem (a $\gamma$ of $\frac{8}{5}$ was chosen). 


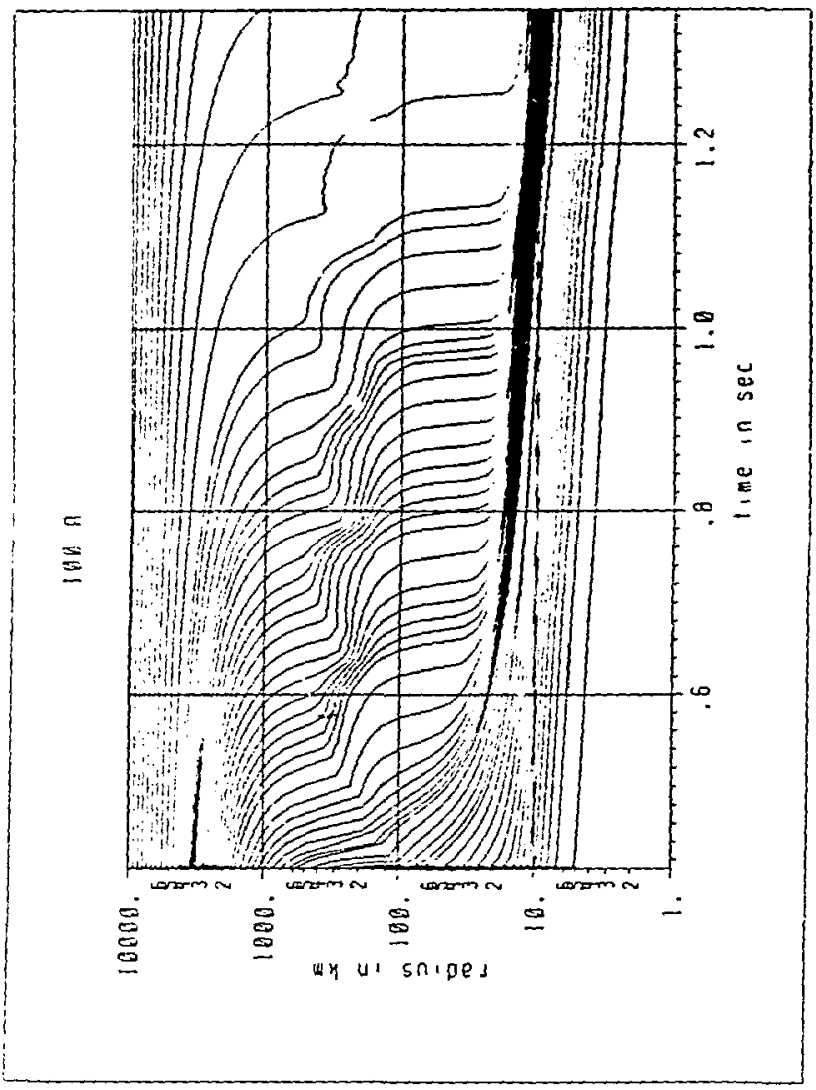

FIGUরe 1a 


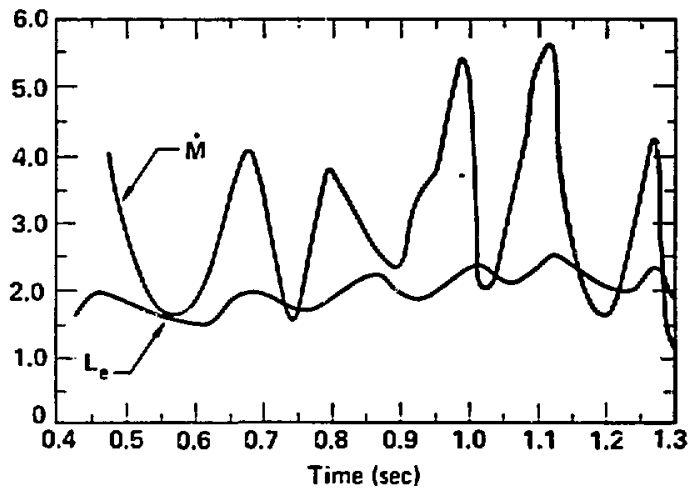

FIGURE Ib 


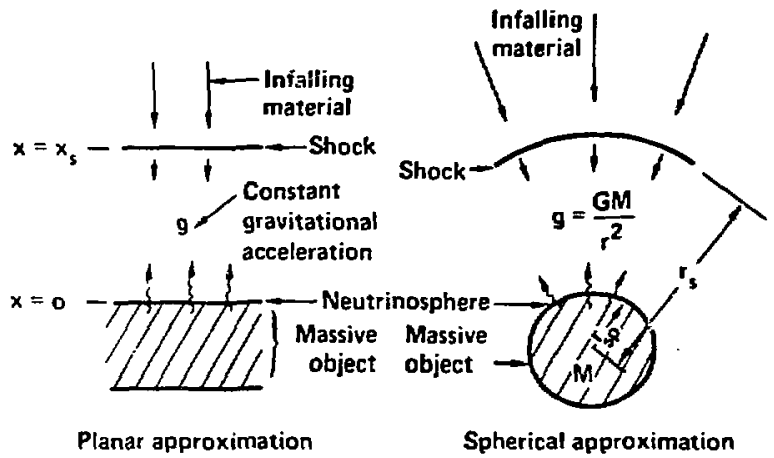

FIGURE 2 


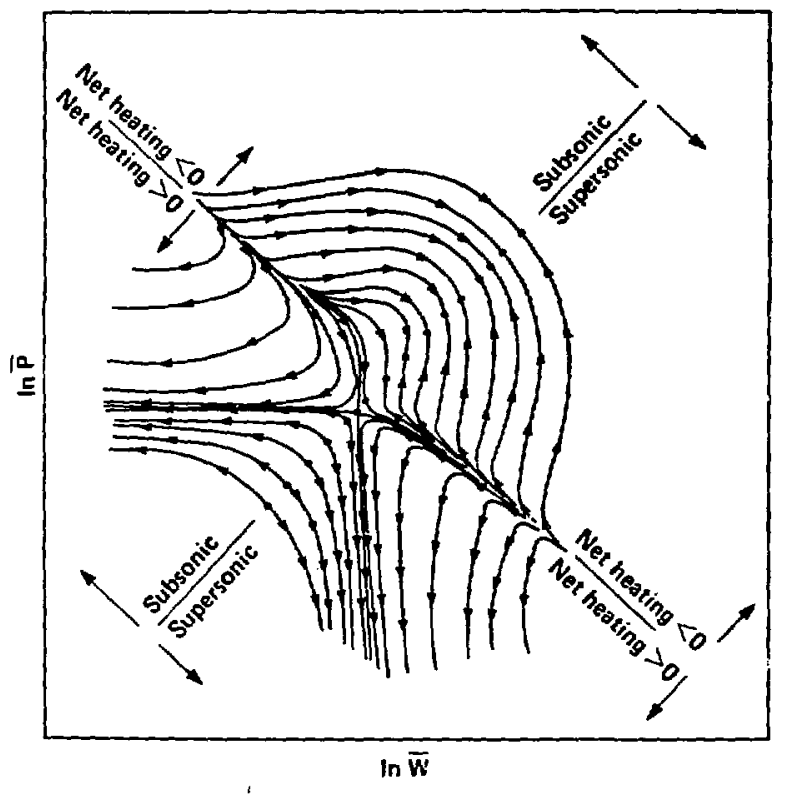

FIGURE 3 


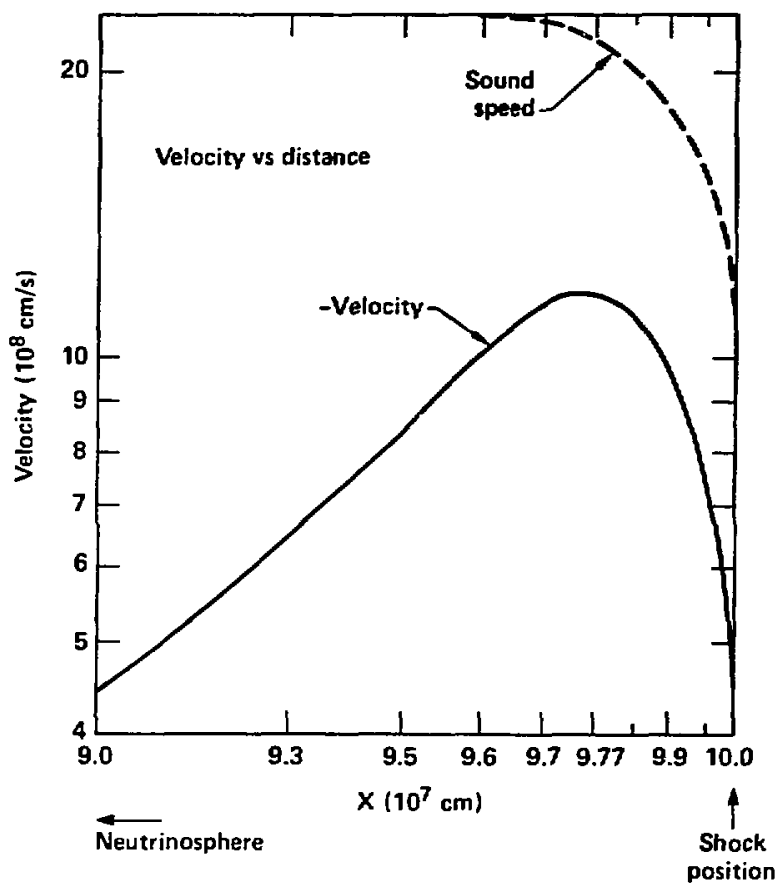

FI GURE $4 a$ 


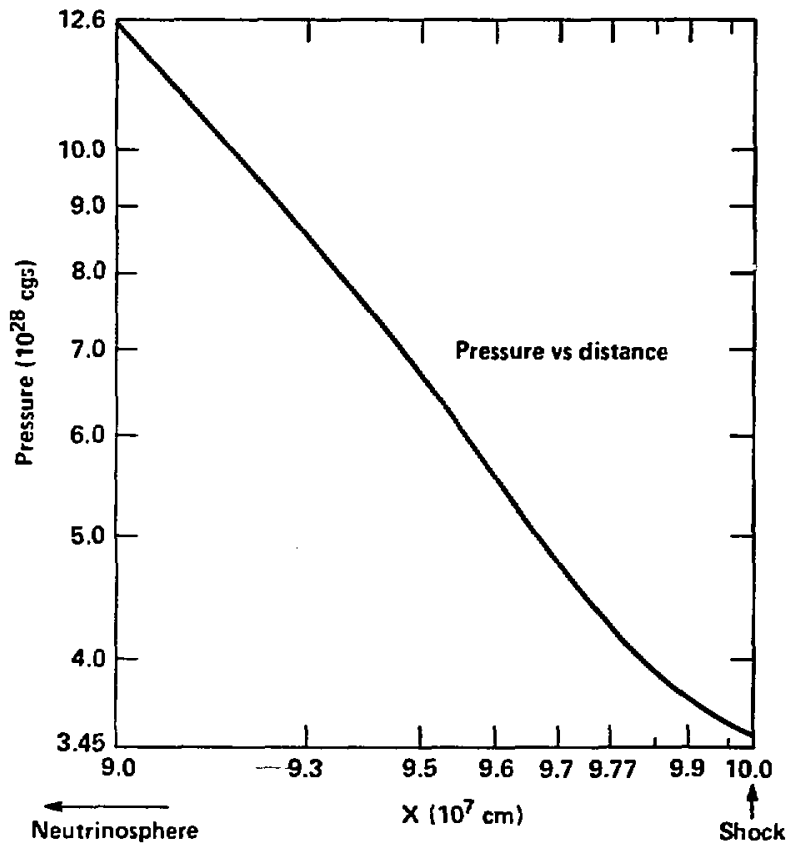

FI GURE $4 \mathrm{~b}$ 


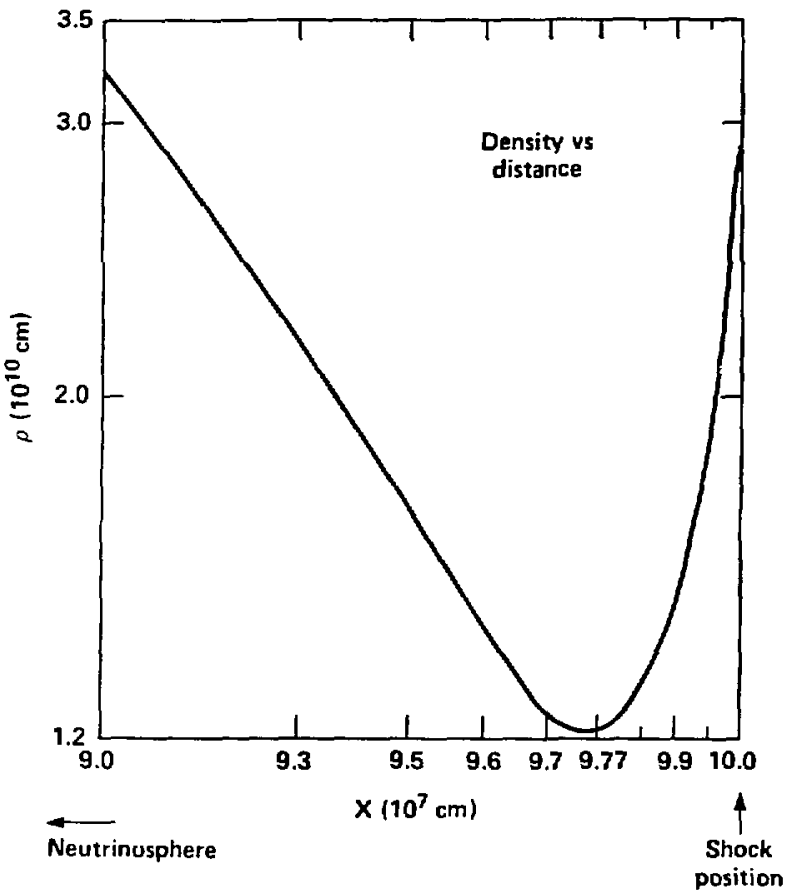

FIGJRE 4C 


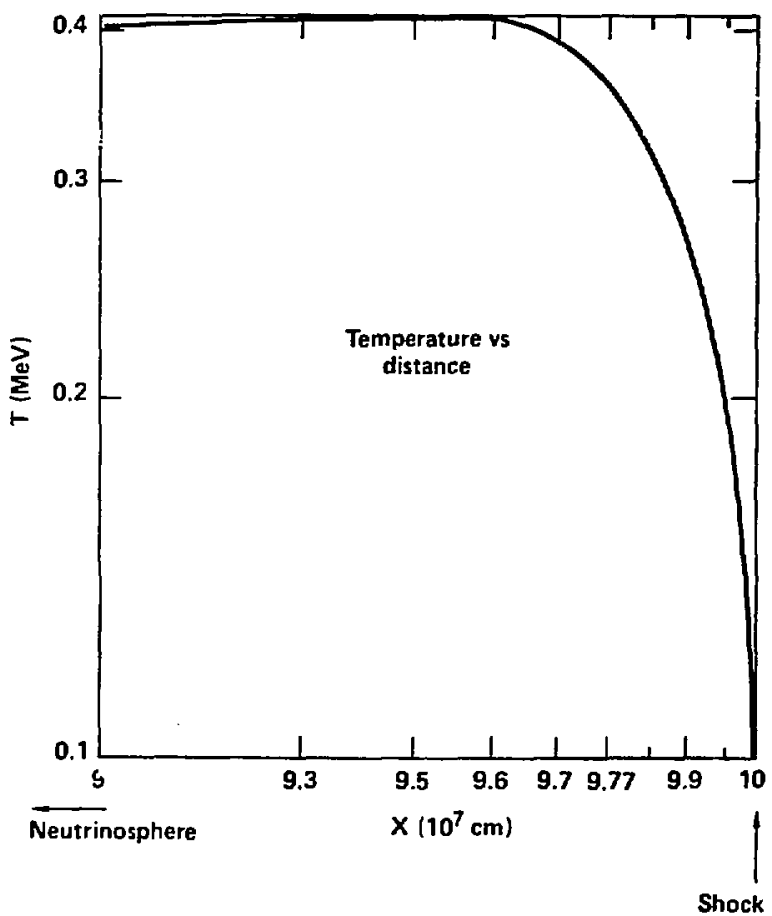

FI GURE 4d 


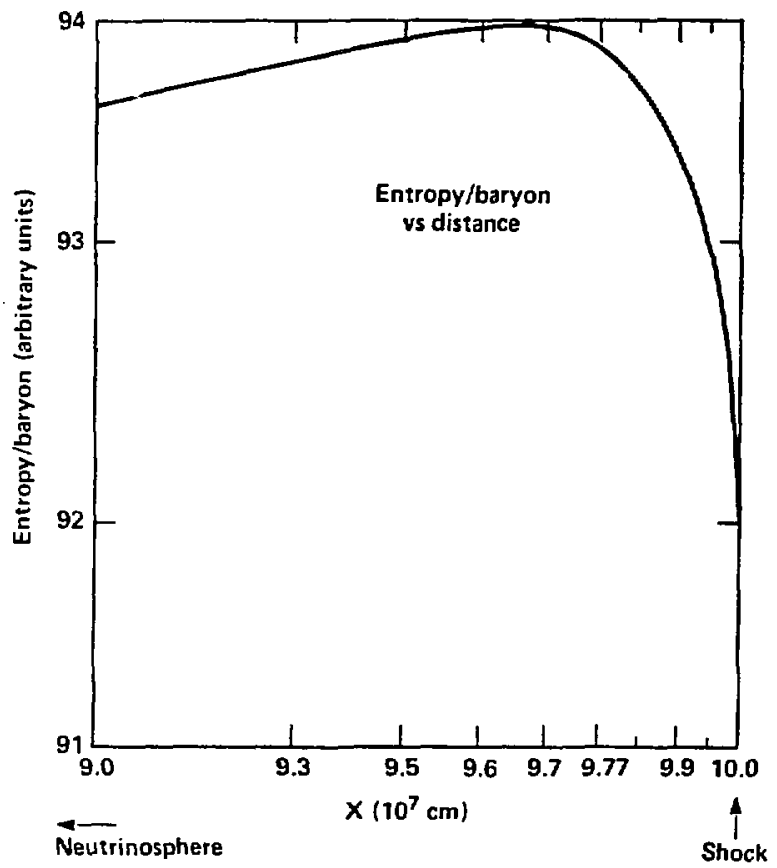

FIGURE $4 \mathrm{e}$ 


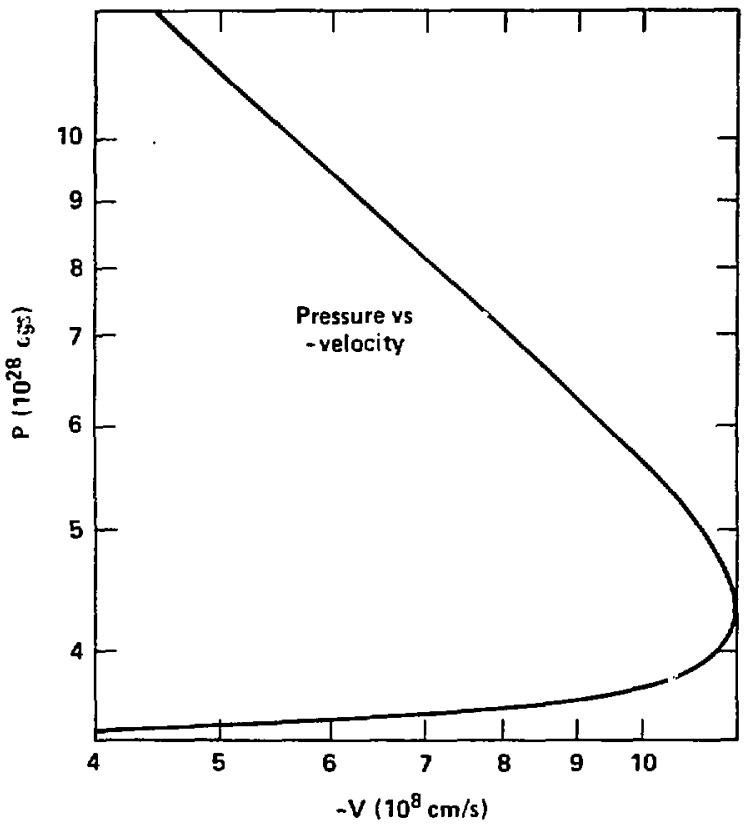

FI GURE $4 f$ 


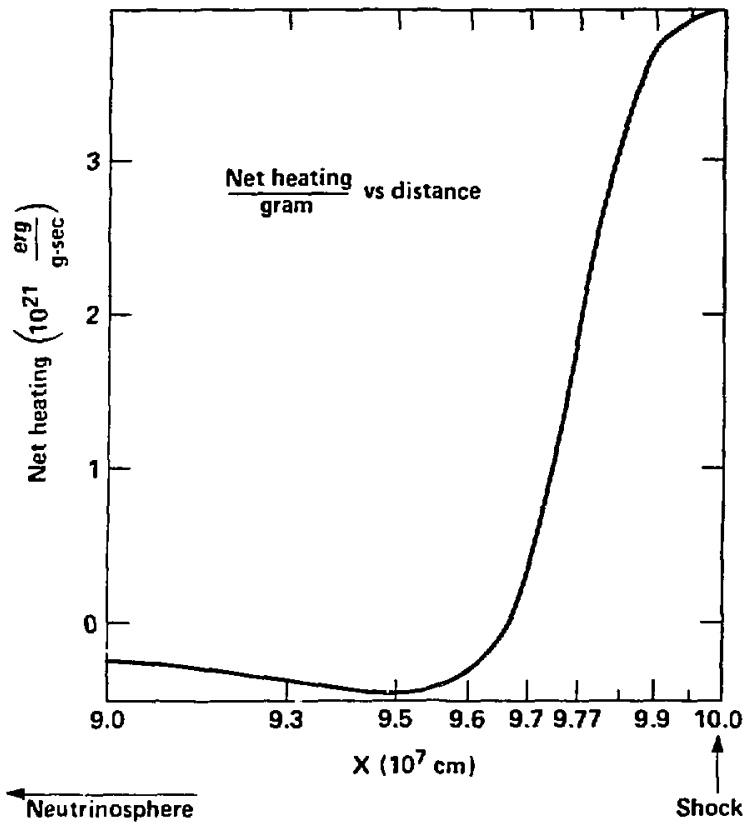

FI. GUU 48 


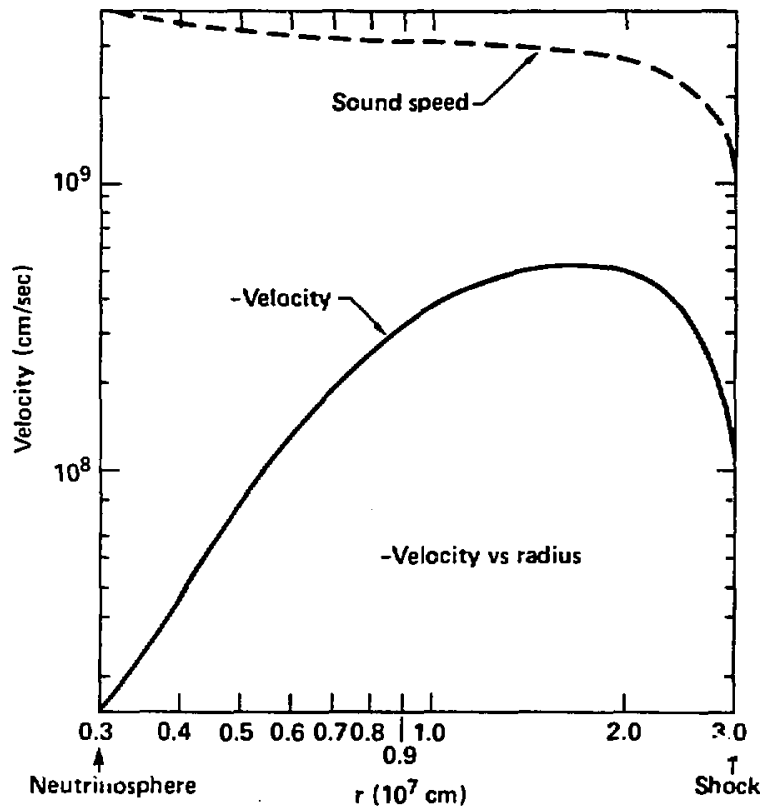

FIGURE $5 a$ 


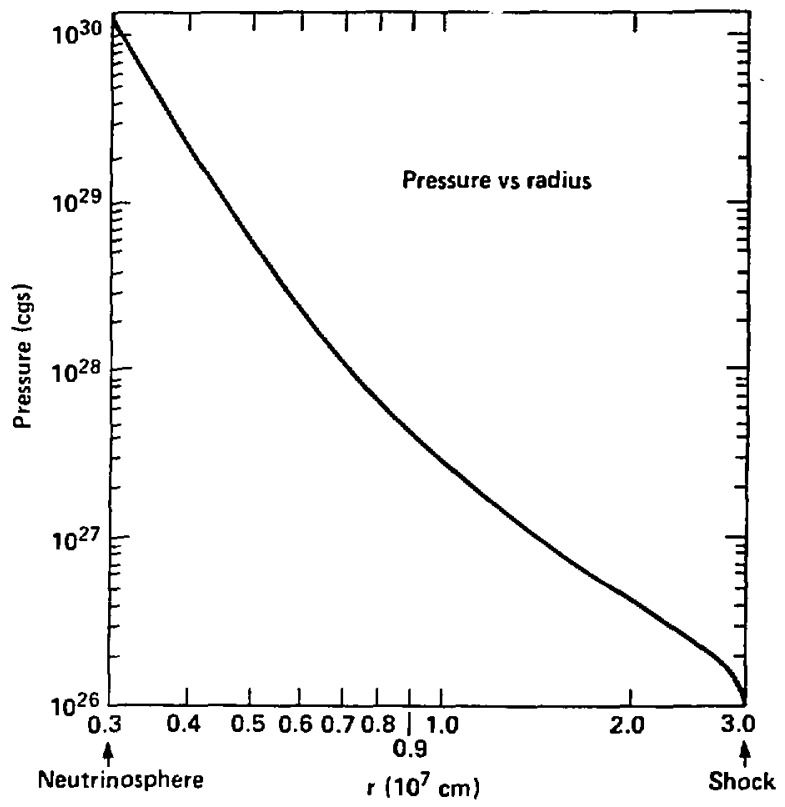

FI GURE $5 \mathrm{~b}$ 


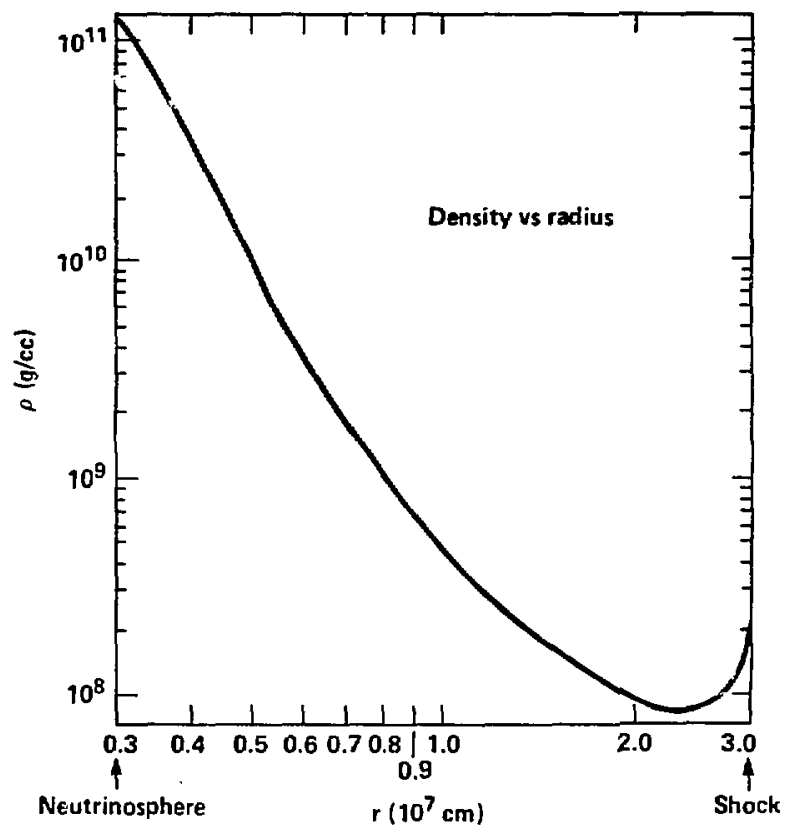

FIGURE 5c 


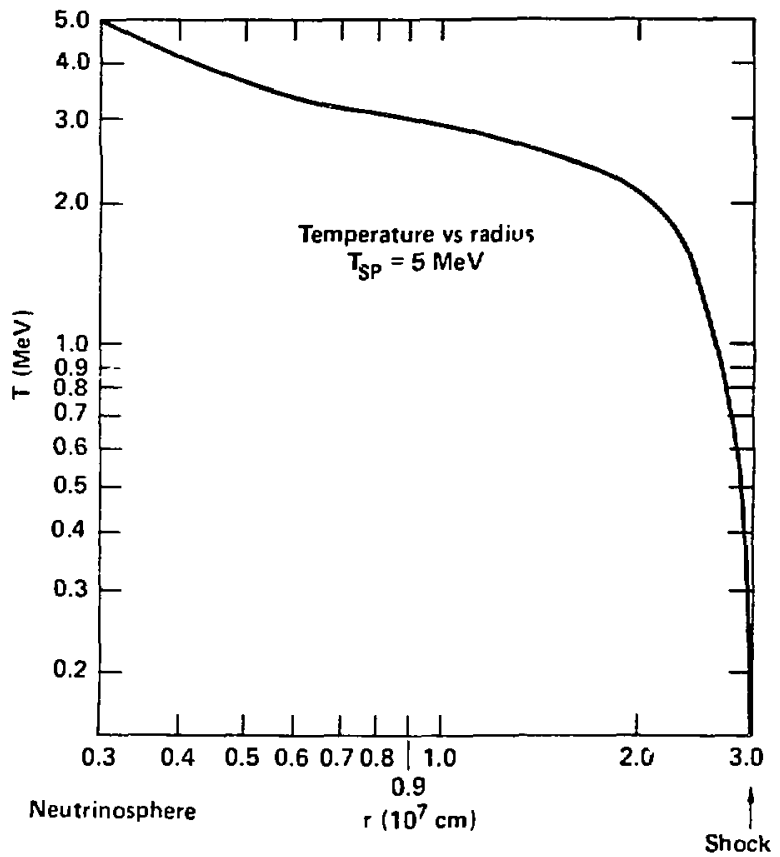

FI GUPE 5d 


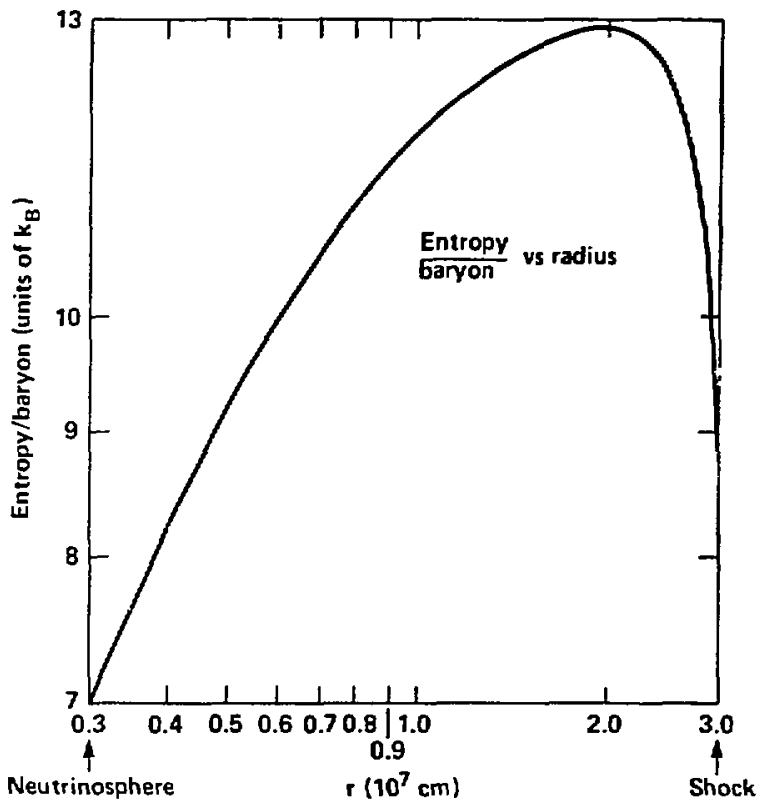

FIGURE $5 \mathrm{e}$ 


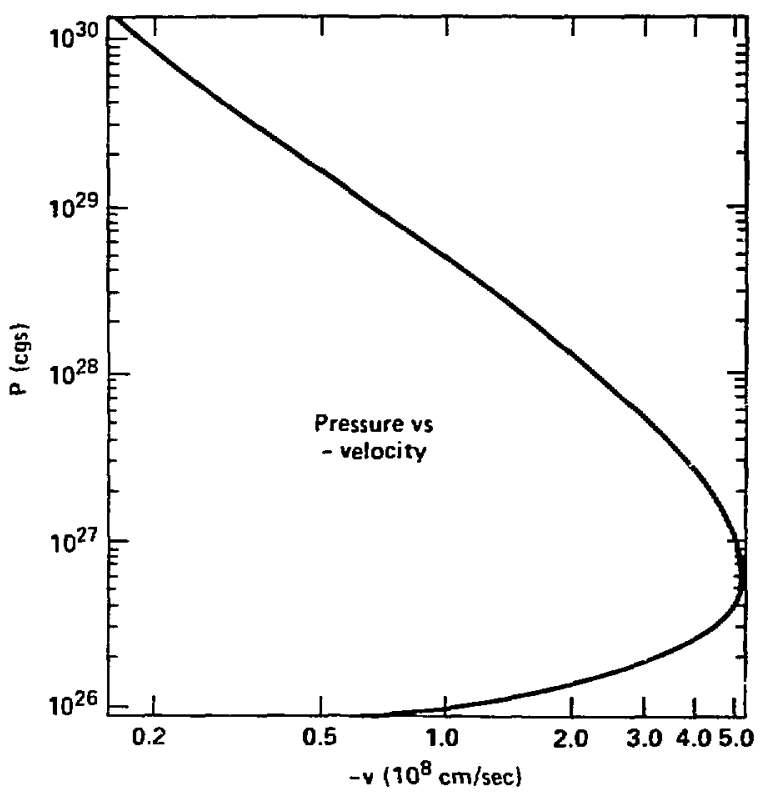

FIGURE $5 f$ 


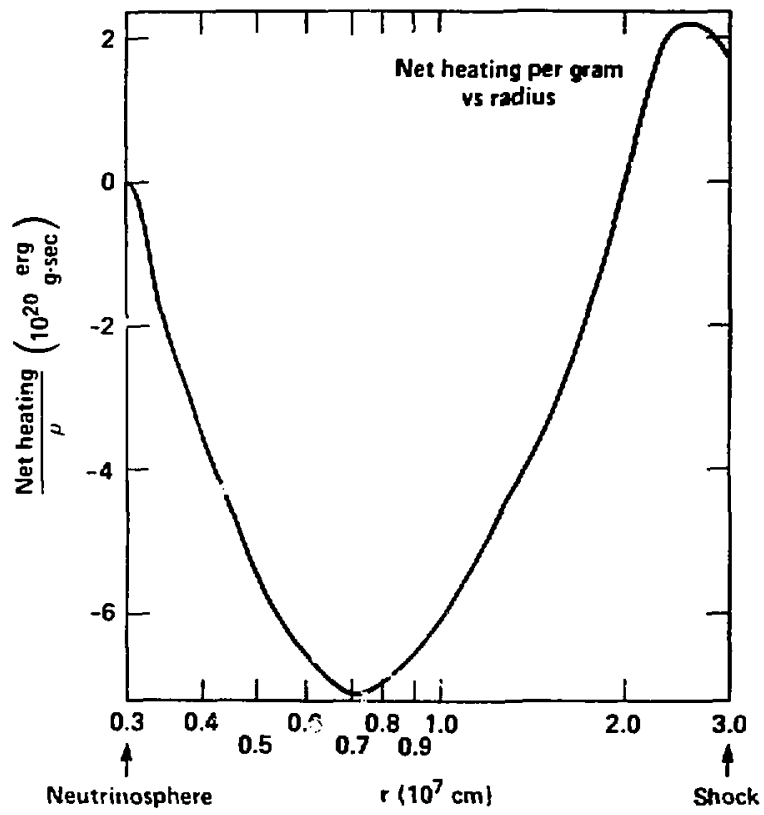

FI GURE $5 E$ 


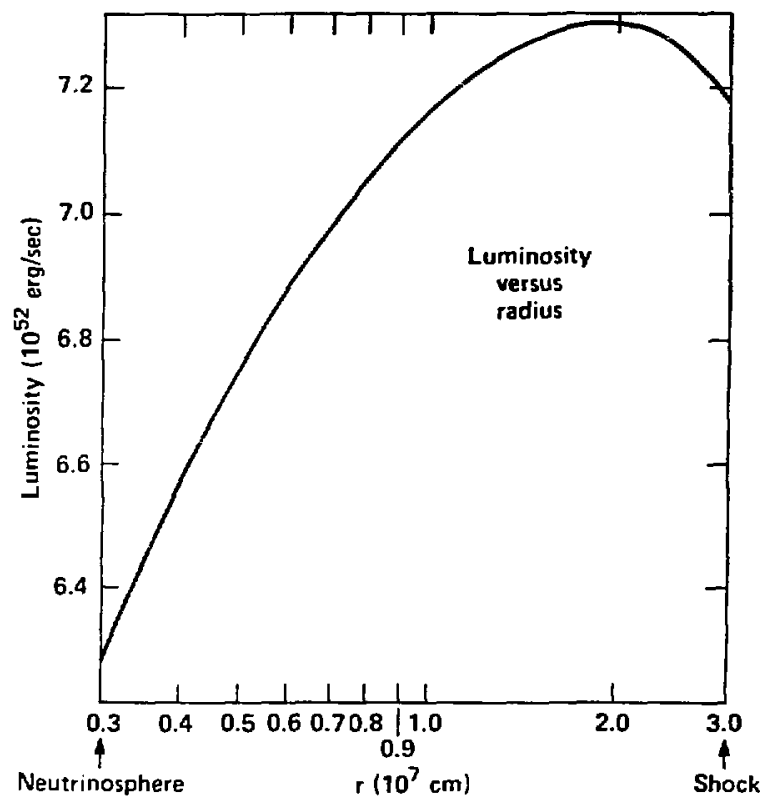

FIGURE 5h 


\section{Chapter VII}

\section{SUPERNOVA CODE RESULTS}

\section{INTRODUCTION}

In this chapter, work I did in collaboration with other authors will be discussed. The papers that resulted may be consulted for a more complete report of the reaearch done. In the second section of this chapter, some briel remarks on preliminary results from the inclusion of convection in the supernova code are made. Also presented is the result from a computer run in which a neutrino redshift was included in the aupernova evolution. Finally, a summary of my contributions to supernova research will be made, along with my opinions on the state of the understanding of collapse driven supernova.

\section{Collaborative Efforts}

Gravitational Collapse and the Cosmic Antineutrino Beckground Woosley, Wilson and Mayle 1985, Ap. J., accepted for publication

The possibility exists that current large detectors being used to search for proton decay could also be used to detect supernova. Antineutrinos produced during gravitational collapse could be detected on the earth by the reaction $\bar{\nu}_{c}+p \Longrightarrow e^{+}+n$. With the detection of both the positron and the neutron, low fluxes of antineutrinos could be measured. This fact led to an investigation of the electron antineutrino spectrum produced by collapsing stars in general, whether or not an explosion occured, since in the process of collapse, neutrinos of all types are produced due to the high temperatures and densities involved.

The higher the antineutrino snergy, the larger the cross-section for interaction, so spectral information is important. The supernova code, with multi-group flux limited diffusion, could provide this spectral information.

A study was undertaken of the antineutrino spectrum produced by the collapse of stars ranging in mass from $10 M_{\odot}$ to $5 \times 10^{5} M_{\odot}$. The hardest neutrinos were found to be produced by stars less than $100 M_{\odot}$ in mass, probably in the mass range $8 M_{\odot} \leq M \leq$ $100 M_{\odot}$. The average antineutrinc energy produced is about $15 \mathrm{Mev}$. However, stars in this mass range produce rare supernova events, so an actual detection of a single supernova exploding is unlikely, while the time integrated production of electron antineutrinos is also limited for these stars by nucleosynthetic constrains. 
The larger mass atars, in the mass range $M \geq 100 M_{\odot}$, produce average antineutrino energies less than about $5 \mathrm{Mev}$. The main reason for the drop in everage energy, as compared to the lower mass atars, is that the neutrinoephere, if it develops, is eaten by the black hole that forms in the center of the atar. The lower mass stars, upon collapse, produce dense cores that may or may not collapse further to black holes. The cores last long er gh to provide for much neutrino emission, even if a black hole eventually forms. The antineutrinos escaping from the neutrinosphere in the atars in the lower mass range have energies about 3 times the neutrinosphere temperature in energy units. The neutrinos produced in the stars with $M \geq 100 \odot$ produce a large fraction of the antineutrinos from volume emission in optically thin regions.

There is a terrestrial background of antineutrinow produced by the natural radioactivity of the earth below about $3 \mathrm{Mev}$. This makes detection of antineutrinos form extraterrestrial sources impossible for energies below about $5 \mathrm{Mey}$. The conclusion was reached that present day detectors would be hard pressed to identify antineutrinos from gravitational collapse. However, in the design of future detectors, this work is useful in that it produced some numbers for experimenters to consider in their planning.

In order to so this research, the supernova code needed modification. Previous to this time, the supernova code had studied collapsing stars in the mass range $10 M_{\odot} \leq M \leq 25_{\odot}$. These stars form dense cores that may or may not collapse to black holes. For the $150 M_{\odot}$, $500 M_{\odot}$ and $5 \times 10^{5} M_{\odot}$ stars, black holes form at the center and continue to accrete the stellar envelope.

I was involved in modifying the supernova code to approximate the formation of a black hole, ad in finding suitable boundary conditions to apply at the boundary of the black hole and the rest of the star. Many different ideas were tried, but I will only describe the method of approximation we finally decided gave the best representation of the actual problem.

A black hole is declared to exist at that time when any sphere of total mass $M$ moves inside twice the Schwarzschild radius for that mass (i.e. $r \leq 2 r_{0}=\frac{1 G M}{\sigma^{2}}$ ). From then on, the central zone becomes the "black hole" (i.e. zone 1). The adjacent zone (i.e. zone 2) is merged into the central zone on each time cycle in which any mass zone in the star is inside twice the Schwarzschild radius for the enclosed mass. The merging process was conducted such that the resulting pressure between zones 1 and the new zone 2 (i.e. old zone 3) was less than or equal to the old pressure between the old zone 2 (merged into zone 1) and old 
zone 3 (new zone 2). This becomes the boundary condition, that the "black hole" produces no pressure on the accreting material. Each time a new zone is accreted, the problem time step is reduced by $40 \%$, in order to help insure the numerical errors introduced by the abrupt merging would not produce instabilities.

The manner of teating the approximation is discusesd next. As mentioned in Chapter III, the approximations used on the supernova code, including gravitational redshift and an enhanced gravitational force (see Chapter III), as well as the black hole accretion were checked against a fully general relativistic code provided by Chuck Evans. Both codes ran the $5 \times 10^{5} M_{\odot}$ star and the results were compared. Doing the comparasion was part of my contribution to the research. It is not a trivial operation, as some modifications to the general relativistic collapse code needed to be made in order to made this type of calculation. The results of this comparasion convinced us that the approximations in the supernova code were very good.

1 was also involved with Jim Wilson in using a known solution to steady state matter accretion onto a black hoke, in order to check the approximations used in the supernova code. The known solution is the general relativistic analogue to the Bondi accretion sojution, called Michel accretion (see Michel (1972)).

All three authors worked in the semi-analytic formulae presented in the resulting paper, to which I refer the reader for compete details.

\section{Stellar Core Collapse and Supernova}

Wilson, Mayle, Woosley and Weaver 1985 XII Texas Symp. Rel. Ap., in press

For the first time, stars in the mass range $11_{\odot} \leq M \leq 100 M_{\odot}$, with the possible exception of the $100_{\odot}$ star, were shown to explode at the end of their stable evolution. These supernovae were al! late time supernovae, where neutrino heating of the matter behind the shock produces material pressure that helps the shock move outward ints the stellar envelope. Explosive energies are found to be consistent with the experimental value of $10^{51} \mathrm{erg}$. Also, the formation of a neutron star remnant of about $1.4 M_{\odot}$ is seen to be produced by stars in the mass range less than about $20 M_{\odot}$. Stars in the mass range above this may produce blackholes as well as a supernova display. Three progenitor stars, all of initial mass $25 M_{\odot}$, but with widely varying iron core masses going into collapse, became supernova. The variance in the size of the iron cores reflected differences in the physics used 
to evolve the stars up to the point of collapse. The late time heating mechanism is thus shown to be fairly insensitive to the exact details of the stellar evolutionary calculations. The nucleosynthesis produced by one the aupernova, the most recent calculation of the $25 M_{\odot}$ star done Weaver and Woosley, was found to be in good agreement with colar abundances.

The oscillations in the neutrino luminosity and the mass accretion rate first seen by Jim Wilson (see Wilson (1985)) were again seen in some of the supernovae studied for the Texas Symposium. A qualititive explanation of the origin of the instability is also given in the Texas paper.

The scenario for the late time supernova can be found discussed in Chapter II of this thesis, and the qualitative explanation of the neutrino luminosity can be found in the sntroduction to Chapter VI.

Neutrinos from Gravitational Collapse

Schramm, Mayle and Wilson 1985 Proc. First International Symp. on Underground Phys.,in press

Neutrinos from Gravitational Collapse

Majle, Wilson and Schramm 1985 Ap. . , submitted for publication

The first paper listed contains some preliminary results on the production of neutrinos of all types by collapsing stars in the mass range $12 M_{\odot} \leq M \leq 100 M_{\odot}$. The second paper presents the results of spectral calculations, as well as a discussion of the method of calculation (i.e. flux limited diffusion). The neutrino production is of interest to particle exferimenters thai are planning to attempt detection of neutrinos form extraterrestial sources. If detection of neutrinos from supernova becomes possible, it is pointed out in the first paper, $t$. $t$ the Wilson delayed supernova mechanism, could be verified, if oscillations in the neutrino luminosity were observed on time scales of 1 second.

Part of the material in Chapter IV is contained in these papers, and the reader is referred to this chapter for a more thorough discussion of the neutrino production found during collapse. 
The calculations tuat were done for the XII Texas Symposium on Relativistic Astrophysics did not include a gravitational redshift or a model for convection. Figures 1a through 1c show mass point trajectories for a $25 M_{\odot}$ atar (identified as $25 \mathrm{C}$ in Wilson et al. 1985). Figure 1a shows the evolution without convection or redshift. Figure $1 \mathrm{~b}$ is the evolution of the same star with a convection model included in the phywico but no redshift. The model for salt finger convection was used. Figure $1 c$ is the $25 M_{O}$ with a redshift included but no convection. As expected, the model with convection has an enhanced neutrino emission due to the dredge up of neutrinos from underneath the neutrinosphere. Figure 2 shows a graph of $Y_{P}$, the proton fraction for the stars shown in figure $1 \mathrm{a}$ and $1 \mathrm{~b}$. As can be seen, the negative gradient in $Y_{P}$ in the model without convection is smoothed out in the model with convection. The neutrino enhancement is only modest, however, with about a $30-50 \%$ increase in luminosity. The explosive energy of $2.0 \times 10^{51}$ ergs was correyjondingly about $30 \%$ higher than the model without convection, which gave $1.6 \times 10^{31}$ ergs when the additional opacity due to electron-positron pair scattering was included. The run of the $25 M_{\odot}$ with a redshift of the neutrinos, is, as expected, a weaker explosion. The explosive energy of $.7 \times 10^{51} \mathrm{crgs}$ is roughly $40 \%$ less than the model without redshift.

\section{SÜMMARY}

\section{Contributions in Supernova Research}

The material in Chapters IV, V, and VI is essentially my own research, as well as the material in Ch:pter III covering the approximate manner in which the general relativistic red shift is taken into account. I also contributed to the csilaborative work summarized in the first part of this chapter. Other contributions have been made in improving or adding additional physics to an already established computer code. These include: improving the electron-positron equation of state, adding pair and plasma source and sink terms to the electron neutrino and antineutrino transport, improving the electron-positron ocattering formul $、$ by refitting the results of Tubbs and Schramm (1975) with the newest value of $\sin ^{2} \theta_{W}=.21$, and recognizing that the computer corle I inherited did not correctly count the total number of electrons and positrons as scattering centers for neutrino interaction.

\section{Collapse Driven Supernova}

Unless our understanding of the equation of state of matter above nuclear densities is mistaken, and the compressibility modulus is around $140 \mathrm{Mev}$ rather then near the laboratory experimental value of $220 \mathrm{Mev}$, the discovery of the explosive mechanism for collapse 
driven supernova has been found. Agreement with observed output energy, nueleangnthesis, and remnant size strengthens the case for the Wilson heating mechaniam.

There are uncertainities in the stellar evolutionary models, such as nuclear reuction rates or electron capture rates that can change the evolutionary acenario (see Wilson et al. 1985) and the resulting progenitor configuration. This in turn can effect the final nucleosynthesis or the output energy; however, vecertainites in atellar evolution do not aeen to drastically effect the chances of an explosion occuring by late time neutrino heating.

The late time supernova mechanism, in which heating of the envelope behind the shock is important to the success of the explosion, can be studied without dealing with uncertain physics (i.e. a poorly known equation of state). The physical processes are well known in the heating region. This then brings up the question of numerics.

Full multi-group multi-angle solution of the Boltzmann equation $f$ "rtrino transport takes about an order of magnitude more computer time than multi-, flux limited difiusion. For som 'the computer simulations reported in this chapter, a run of 2 hours on a Cray was nivessary to follow about 1 second of supernova evolution. The neutrino transport subroutines currently use about $60 \%$ of the CPU time consumed in a run. At present, computer time constrairts force approximate $t_{2}$ ansport methods.

The work reported in Chapter VI is a step in the direction of trying to understand the late time heating mechanism without a complete supernova time evolution. I believe this is a good approach, and hope further work will result in a betler uncerstanding of late time supernova. 


\section{Chapter VII References}

Michel, F.C. 1972, Ap. and Spac. Sci., v15. 153.

Wilson, J.R. 1985, in Numerical Astrophysics, ed. J. Centralla, J. LeBlane, and

R. Bowers, (Jones and Bartlett:Boston), p. 422. 


\section{Chapter VII Figure Captions}

Fig. 1 Mass point trajectories for the 25 solar mass supernova. These figures show the effect of convection and a gravitational redshift.

Fig. 2 Proton fraction versus interior mass in the 25 solar mass star. The figure compares the evolution with and without convection. 


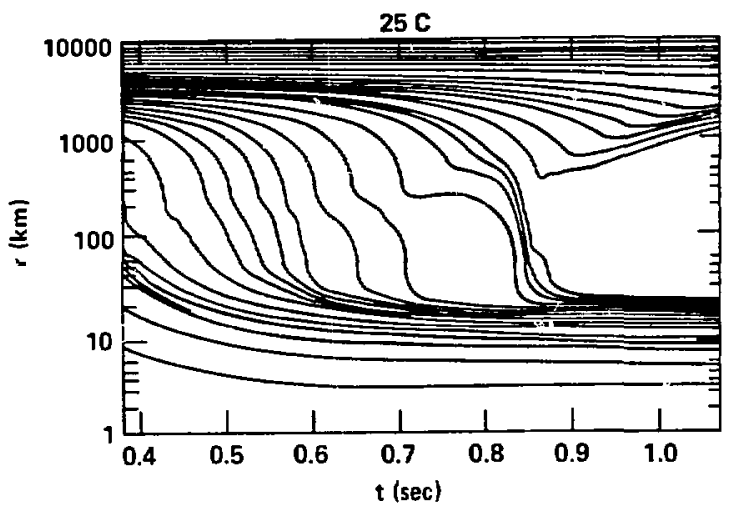

FIGURE la 


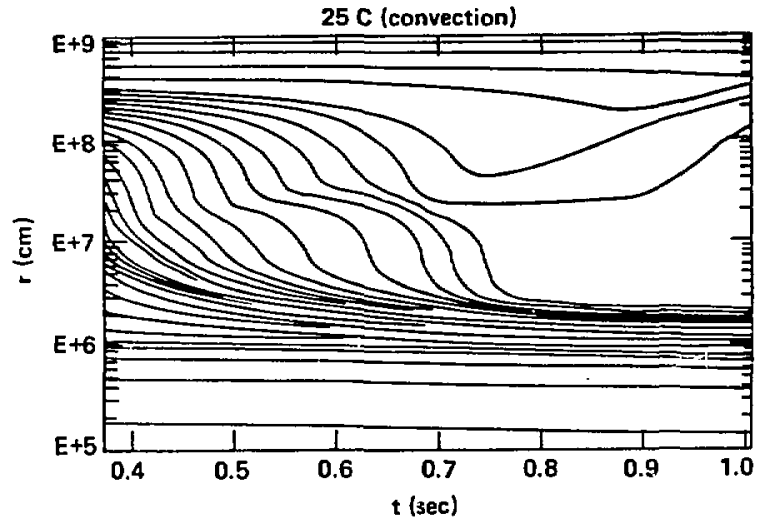

FIGUR 16 


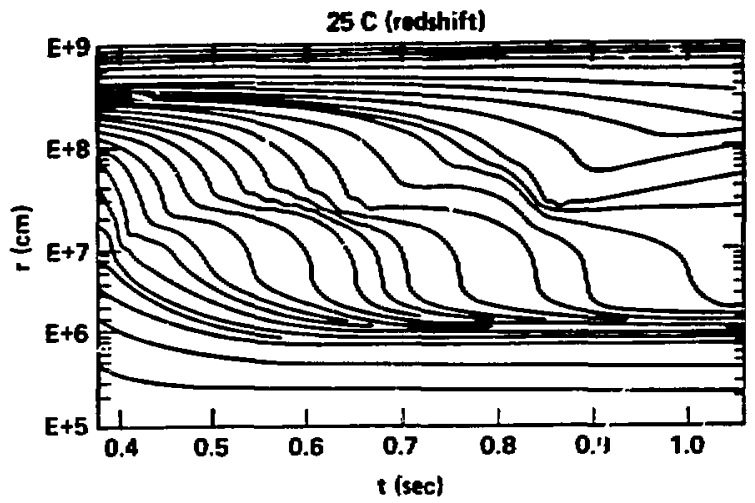

FIGURe Ie 


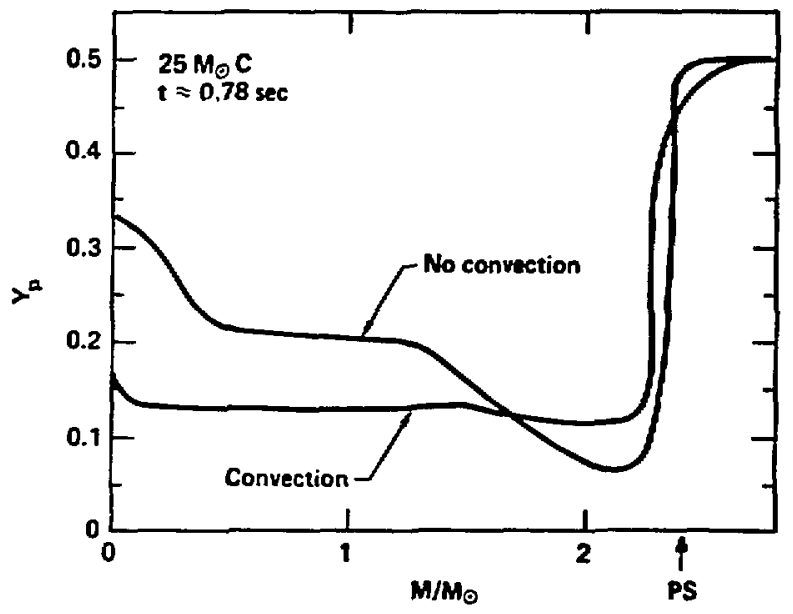

FI GURE 2 


\section{Appendix A}

\section{INTRODUCTION}

The complete net of equations needed to dexcribe the evolution of a mpernora include:

\section{Einatein ' equation:}

2. Equations that guarantee the conservation of:

(a) matter + neutrino strem energy

(b) electric cherge

(c) baryon number

(d) lepton number

3. Boltzmann equation for neutrino

\section{I.latter equation of atate}

The purpose of this appandix is to translate the necessary equations described above into mathematical form. In the first ection, the evolution equations are displayed and briefly discussed. The only asoumption made is that matter is described by an ideal fluid. In the second section, the additional asumption of spherical symmetry is introduced.

\section{SECTION I}

In the following, letters at the beginning of the Latin alphabet, such as $a, b, c$, denote spacetime indices of tensors. Letters near the middle of the Latin alphabet, such as $i$, j, $k$ represent space indices. At the end of thi : appendix, a list of the notation, in the approximate order in which it was introduced, ean be found.

\section{EINSTEIN EQUATIONS}

In units where $G=\frac{1}{8 \pi}, c=1$, the Einstein equations are:

$$
G_{a b}=T_{a b}+t_{a b}
$$

where $T_{a b}$ is the matter atress energy tensor, and $t_{a b}$ is the neutrino stress energy tensor. Four of the 10 equations (1) are not evolution equations, but constraint equations, as they contain only first derivatives of the metric with respect to the timelike coordinate (see 
Weinberg (1972)) . If the initial data eatisfy the constraint equations at one time, the 6 evolution equations guarantee the constraint equationa are zatisfied thereafter.

The $3+1$ formalism of Arnowitt, Deser, and Misner described in Misper, Thorne, and Wheeler (1973) (hereafter denoted by MTW), and furtber refined by Yosk (1979), provide - natural way to meparate the 4 conatraint equationa from the 6 dynamical equations. The idea is to break apacetime into apace plus time, and then write sab and $G_{\text {at }}$ in terms of geometrical objects describing the particular way apacetime is aliced up into 3 dimensional bypersurfaces.

From differential geometry, two 3 dimensional tensora describe an embedded surface in a 1 dimensional space. $\Lambda_{i j}$, the metric tensor in the alice, determines the intrinsic curvature of the surface, independent of the surrounding manifold. $K_{i j}$, the extrinsic curvature tensor, describes the embedding of the auface in 1 dimensional spacetime.

The 3 dimensional surface determines a rector field, $n^{*}$, of unit timelike normals (i.e. $\left.n_{a} n^{a}=-1\right) . n^{a}$ can represent the 4 velocity of a phyaical observer, called the Eulerian observer, who is at rest in the slice. In the following, the way is which gat and $G_{a b}$ are expressed in terms of $K_{i j}, \Lambda_{i j}$, and $n^{*}$ is sketched.

$A_{a b}$ is defined es follows:

$$
\Lambda_{e b}=g_{e t}+n_{0} n_{b}
$$

$\Lambda_{a l}$ is really a spatial metric since $\Lambda_{a b} n^{b}=0$. In tact, since $\Lambda_{a b}=\Lambda_{b a}$ and $\Lambda_{\Delta b} n^{b}=0$, the 16 components of $\Lambda_{a b}$ are all expressible in terms of only 6 , which are taken as $\Lambda_{i j}$.

$G_{a b}$ is constructed with ${ }^{4} R_{b e d}^{a}$, the 4 dimensional Riernann tensor. The 256 components of ${ }^{4} R_{b c d}^{a}$ can be expressed in terms of only 20 independent components (see MTW). Fousteen of the 20 can be expressed in terms of $\lambda_{i j}, K_{i j}$, and their spatial derivatives (i.e. $D_{i}$, the covariant derivative compatible $\dot{s}_{i j}$ ), using the equations of Gauss and Codazzi. (The Gauss-Codazzi equations are integrability conditions that $\Lambda_{i j}$ and $K_{i j}$ must satisfy if they are to describe a surface that can actually be constructed in spacetime.)

These 14 components of ${ }^{4} R_{\text {ded }}^{a}$ are all that is needed to form the following 4 components of $G_{a b}$ :

$$
\begin{gathered}
n^{a} n^{b} G_{a b}=\frac{1}{2}\left(s_{R}+\left(K_{i}^{i}\right)^{2}+K_{i j} K^{i j}\right) \\
-\Lambda_{i}^{a} n^{b} G_{a b}=D_{j} K_{i}^{j}-D_{1} K_{j}^{j}
\end{gathered}
$$


where ${ }^{3} R$ is the Ricci acalar compatible with $\Lambda_{i j}$ Now using $G_{a b}=T_{a b}+t_{a b}$ in the above, the 1 Einstein constraint equations are found (ee York (1979) for a more complete discussion):

$$
\begin{gathered}
{ }^{3} R+\left(K_{i}^{i}\right)^{2}-K_{i j} K^{i j}=2 \rho H=H \text { amiltonian constraint } \\
D_{j} K_{i}^{j}-D_{i} K_{j}^{j}=J_{i}=\text { momentum constraint }
\end{gathered}
$$

where:

$$
\begin{aligned}
& \rho_{H}=n^{c} n^{b}\left(T_{a b}+t_{a b}\right) \\
& J_{i}=-A_{i}^{c} n^{b}\left(T_{a b}+t_{a b}\right)
\end{aligned}
$$

$\rho_{H}$ is the energy density and $J_{i}$ is the momentum density as meen by the Eulerian observer.

Before writing the remaining 6 evolution equations, the manner in which the spatial coordinates are to be moved from alice to slice must be opecified. This is called a gauge freedom.

Define a vector field $t^{a}$ such that:

$$
t^{\bullet}=\alpha n^{\bullet}+\beta^{\bullet}
$$

with $\hat{\beta}^{a} n_{a}=0$ (i.e. $\beta^{a}$ is really a spatial vector). The trajectory of constant spacelike coordinates will travel along $t^{*}$, as is illustrated in figure $1 . \beta^{i}$ represents the freedom to - perform a special relativistic Lorentz transformation relative to the Eulerian observer, and $\alpha$ adjusts coordinate time so that adt is the proper time as measured by the coordinate observers.

$\alpha$ is known as the lapse function, and $\beta^{i}$ is the shift vector. Together, they form 1 kinematical degrees of freedom, and must be opecified independently of Einstein's equations (they are just telling the coordinate observers how to move.)

The 4 dimensional line element can then be written as:

$$
d s^{2}=-\left(\alpha^{2}-\beta_{i} \beta^{i}\right) d t^{2}+2 \beta_{i} d x^{i} d t+\Lambda_{i j} d x^{i} d x^{j}
$$

The remaining 6 components of ${ }^{4} R_{b e d}^{a}$ can now be expressed in terms of $\Lambda_{i j}, K_{i j}, \alpha, \beta^{i}$ and their derivatives. $G_{a b}$ is then determined, and the 6 second order in time Einstein evolution equations can be written down. 
However, it is more convenient to we the following 12 firt order in time equations, equivaleat to the 6 evolution equations:

$$
\begin{gathered}
\frac{\partial K_{i j}}{\partial t}=-D_{i} D_{j} \alpha+\alpha\left({ }^{3} R_{i j}-2 K_{i j} K_{j}^{l}+K_{i}^{l} K_{i j}-S_{i j}-\frac{1}{2} \Lambda_{i j}\left(\rho_{H}-S\right)\right) \\
+\beta^{l} D_{l} K_{i j}+K_{i j} D_{j} \beta^{l}+K_{l j} D_{i j} \beta^{2} \\
\frac{\partial \Lambda_{i j}}{\partial t}=-2 \alpha K_{i j}+D_{i} \beta_{j}+D_{j} \beta_{i}
\end{gathered}
$$

where:

$$
S_{i j}=A_{i}^{e} A_{j}^{b}\left(T_{a b}+t_{a b}\right)
$$

$S_{i j}$ is thus the spatial stresses as measured by the Eulerian obeerver. $S$ is defined to be $S_{i}^{j}$.

For completeness, the constraint equations are repeated below. As stated previously, the above 12 first order evolution equations guaranlee the constraints are antisfied, provided they are satisfied at one time.

$$
\begin{gathered}
{ }^{3} R+\left(K_{i}^{i}\right)^{2}-K_{i j} K^{i j}=2 \rho_{H} \\
D_{j} K_{i}^{j}-D_{i} K_{j}^{j}=J_{i}
\end{gathered}
$$

\section{BARYON NUMBER CONSERVATION}

Defining $n_{B}$ as the number of baryons per unit volume as measured in a frame where the matter is locally at rest, the equation that implies baryon number conservation is:

$$
\left(n_{B} u^{\bullet}\right)_{; a}=0
$$

where $u^{a}$ is the matter 4 velocity.

Neglecting the electron mass as compared to the baryon mass, the matter rest mass density, $p$, is proportional to $n_{B}$, the constant of proportionality being the baryon mass. Therefore, ( 7$)$, can be replaced by:

$$
\left(\rho u^{a}\right)_{i a}=0
$$


Equation (B) will be rewritten in a more phyacally transparent form, wing $D$, the density measured by the Eulerian obeerver, and $v^{i}=v^{i}=4$, the 3 velocity of the luid a measured by the coordinate observer (see Wileon(1979), Shapiro and Teukoloky(1980)).

The fluid 3 velocity, $v_{E}^{i}, \approx$ meanured by the Eulerian obeerver in found to be:

$$
v_{i}^{i}=\frac{v^{i}+p^{i}}{\alpha}
$$

The figure 2 illustrates the above. Now defining $w=-n^{4} u_{s}$, it can be thown (using $u_{a}=g_{a b} u^{b}$ and $u^{*} u_{a}=-1$ ) that:

$$
u=\frac{1}{\sqrt{ }\left(1-\frac{\left(v_{i}+\beta_{i}\right)\left(v^{j}+\beta^{i}\right)}{a^{2}}\right)}=\frac{1}{\sqrt{ }\left(1-v_{E}^{i} v_{E i}\right)}
$$

$u$ is thus a generalization of the npecial relativiatic gamma that relates physical quanities measured in two frames, the fluid frame and the Eulerian frame.

Therefore, $D=u \rho$, and equation (8) becomes:

$$
\frac{\partial D}{\partial t}+D \frac{\partial \ln \Upsilon^{\frac{1}{2}}}{\partial t}+\frac{1}{\Upsilon^{\frac{1}{2}}} \frac{\partial}{\partial x^{i}}\left(r^{\frac{1}{2}} v^{i} D\right)=0
$$

where $\Upsilon^{\frac{1}{2}} \equiv \sqrt{\text { Det } \Lambda_{i j}}$ (note that $\sqrt{-\operatorname{Det} g_{a b}}=\alpha \Upsilon^{\frac{1}{2}}$ ).

In the nonrelativistic limit (9) reduces to:

$$
\frac{\partial \rho}{\partial t}+\nabla \cdot(\rho \vec{v})=0
$$

\section{STRESS ENERGY CONSERVATION}

The matter stress energy tensor, $T_{a b}$, will be taken in the ideal fluid approximation:

$$
T^{a b}=\left(\rho+e_{m}+P_{m}\right) u^{a} u^{b}+P_{m} g^{a b}
$$

where $e_{m}$ is the matter internal energy density and $P_{m}$ is the matter pressure, as seen by an observer at rest with respect to the matter fiuid. This is an excellent approximation for the supernova collapse, as viscosity and heat conduction due to the finite mean free path of the matter particles is only important in shock fronts, where material properties can change over distances on the order of the mean free path.

The neutrino stress energy tensor, $t_{a b}$, is defined as:

$$
t^{a b}=\int p^{a} p^{b} \int d P
$$


where $f$ is the neutrino distribution function, $d P$ is the invariant 3 morrentum rolume, and $p^{*}$ is the neutrino 4 momentum (cee Lindquint (1966)). Six neutrino feld are coneidered, $\nu_{\varepsilon}, \bar{\nu}_{e}, \nu_{\mu}, \bar{\nu}_{\mu}, \nu_{r}, \bar{\nu}_{s}$, and $t_{e l}$ will represent the total contribution from all 6 fielda.

The equacion for atreas energy coneervation in:

$$
\left(T_{a}^{+}+t_{i b}^{b}=0\right.
$$

This is a set of 4 equations which oplit into an energy conservation equation, and Euler's equation. The energy conservation is discussed firat. It in taken to be:

$$
u^{*}\left(T_{a}^{b}+t_{a}^{b}=0\right.
$$

Iniroducing the energy density, $E=u e_{m}$, as measured by the Eulerian obeerver, equation (13) becomes:

$$
\begin{gathered}
\frac{\partial E}{\partial t}+E \frac{\partial \ln \Upsilon^{\frac{1}{2}}}{\partial t}+\frac{1}{\Upsilon^{\frac{1}{2}}} \frac{\partial}{\partial x^{i}}\left(E v^{i} \Upsilon^{\frac{1}{2}}\right)+\frac{P_{m}}{\Upsilon^{\frac{1}{2}}}\left(\frac{\partial\left(u \Upsilon^{\frac{1}{2}}\right)}{\partial t}+\frac{\partial}{\partial x^{i}}\left(u v^{i} \Upsilon^{\frac{1}{2}}\right)\right) \\
=\alpha u^{a} t_{a: b}^{d}
\end{gathered}
$$

In the nonrelativistic limit, the sbove beconies:

$$
\frac{\partial E}{\partial t}+\nabla \cdot(\vec{v} E)+P_{m} \nabla \cdot \vec{v}=- \text { change in neutrino energy }
$$

The Euler equation is:

$$
\left(T_{i}^{b}+t_{i}^{b}\right)_{i b}=0
$$

A new 4 vector, $S_{\mu}=\left(\rho+e_{m}+P_{m}\right) u_{\mu}=\left(D+E+P_{m} u\right) u_{\mu}$ is useful in writting (15) in a physically transparent form. Using $S_{\mu}$, equation (15) becomes:

$$
\begin{gathered}
\frac{\partial S_{i}}{\partial t}+S_{i} \frac{\partial \ln \Upsilon^{\frac{1}{2}}}{\partial t}+\frac{1}{\Upsilon^{\frac{1}{2}}} \frac{\partial}{\partial x^{j}}\left(S_{i} v^{j} \Upsilon^{\frac{1}{2}}\right)+\alpha \frac{\partial P_{m}}{\partial x^{i}} \\
+\frac{1}{2} \frac{\partial g^{a b}}{\partial x^{i}} \frac{S_{\alpha} S_{b}}{S^{t}}=-\alpha t_{i ; b}^{b}
\end{gathered}
$$

In the nonrelativistic limit $S_{i}=\rho v^{i}$, and using the nonrelativistic mass conservation equation, (16) becomes, in the absence of a gravitational field:

$$
\rho \frac{\partial \vec{v}}{\partial t}+\rho \vec{v} \cdot \nabla \vec{v}+\nabla P=\text { neutrino acceleration }
$$




\section{LEPTON NUMBER, CONSERVATION}

Lepton number is defined as the number of leptons minus the number of antileptons. The leptons present in a eupernova collape are the electron, electron neutrino, muon neutrino, and tau neutrino. All the antiparticles of the 4 leptome abore are aleo precent. The muon, whose rest masa is $105 \mathrm{Mer}$ would only be produced in the very central region of a cupernova where the equation of atate is poorly known, wo its presence is neglected in the mpernova model studied here. The tau lepton has a rest mae of $1784 \mathrm{Mer}$ and will not be present in a supernovs collape (see the Particle Propertien Data (1964) for the manes quoted).

The equation for lepton number conservation is:

$$
\left(n_{0} u^{*}\right)_{i \cdot}+N_{i a}^{a}=0
$$

where $n_{0}=n_{e^{-}}-n_{e^{+}}$is the difference in the number dennities of electrons and positrons as measured in the fuid reat frame. $N^{*}=N_{\nu_{0}}-N_{\nu_{*}}^{*}$, wher $N_{\nu_{s}}^{*}=\int P^{*} f_{\nu_{0}} d P$ is the number flux in neutzinos of species $\omega . N^{*}$ is thus the difference in the number flux of electron neutrinos and electron antineutrinos. Mu and Thu neutrino and their antiparticles are not able to change lepton number ance the producton of tau particlea is not likely in a supernova, and any muons produced are in a region where matte: is in thermal equilibrium with the neutrinos. In the above $f_{\nu_{*}}$ is the generalized Fermi-Dirac distribution function for the " ${ }^{\text {th }}$ species of neutrino.

Now iniroducing $\bar{Z}$ such that:

$$
n_{0}=\frac{\rho \bar{Z}}{m_{B}}
$$

and using $\left(\rho u^{a}\right)_{; a}=0,(17)$ can be written as:

$$
\frac{D}{m_{B}}\left(\frac{\partial \bar{Z}}{\partial t}+v^{i} \frac{\partial \bar{Z}}{\partial x^{i}}\right)=-\alpha N_{i a}^{a}
$$

In the nonrelativistic limit, (18) becomes:

$$
\frac{\rho}{m_{B}}\left(\frac{\partial \bar{Z}}{\partial t}+\vec{v} \cdot \nabla \bar{Z}\right)=\text { change in lepton number }
$$

\section{CHARGE CONSERVATION}

The equation expressing conservation of electric charge is:

$$
\left(n_{0} u^{a}\right)_{; a}=\left(n_{p} u^{a}\right)_{; a}
$$


where nP is the number of protrons per unit rolume a meacured in the fuid frame.

Introducing $Z$ such that:

$$
n_{P}=\frac{P Z}{m_{B}}
$$

equation (19) becomes:

$$
\frac{\partial \bar{Z}}{\partial t}+v^{i} \frac{\partial \bar{Z}}{\partial x^{i}}=\frac{\partial Z}{\partial t}+v^{i} \frac{\partial Z}{\partial x^{i}}
$$

\section{BOLTZMANN EQUATION FOR NEUTRJNOS}

A generally covariant Boltzmann equation has been derived by Lindquiat (wee Lindg; int(1966)). An abbreviated derivation follows.

Call $f_{\nu_{0}}\left(x^{a}, p^{b}\right)$ the generalized Fermi Dirac diatribution function that dewcribes neutrinos of species 8 . Then:

$$
d f_{\nu_{*}}=\frac{\partial f_{\nu_{0}}}{\partial x^{*}} d x^{*}+\frac{\partial f_{\nu_{0}}}{\partial p^{*}} d p^{\bullet}=\delta f_{\nu_{0} \text { endivion }}
$$

Now assuming the neutrinos move along geodesics between collisions:

$$
\begin{gathered}
\frac{d x^{e}}{d r}=p^{a} \\
\frac{d p^{e}}{d \tau}=-\Gamma_{b e}^{e} p^{e}
\end{gathered}
$$

where the variable $r$ parameterizing the geodesics is normalized so that $\frac{d x^{a}}{d r}=p^{a}$ hoids.

The Boltzmann equation becomes:

$$
p^{a} \frac{\partial f_{\nu_{s}}}{\partial x^{a}}-\Gamma_{b c}^{e} p^{b} p^{c} \frac{\partial f_{\nu_{k}}}{\partial p^{2}}=\delta f_{\nu_{c} \text { collinions }}
$$

\section{EQUATION OF STATE}

The equation of state is discussed in Appendix B. For now, the equation of state is symbolically written as:

$$
P_{m}=P_{m}\left(\rho, \epsilon_{m}\right)
$$

The supernova equations have been written in a generai form in the above. In Section II, the assumption of spherical Bymmerty is introduced, and the supernova equations are rewritten using this additional assumption. 
SECTION II

A 4 dimencional line element that reopects epherieal aymmetry can be writien Wilen(1979)):

$$
d \theta^{2}=-\left(\alpha^{2}-\rho, F\right) d^{2}+2 \beta r d r+A^{2} d^{2}+B^{2} r^{2} d R^{2}
$$

Two different gauge choices will be made, and the compiete zet of aupernove equations will be written for each choice.

Before proceeding further, come counting of gravitational dynamical degrees of freedom can be done. Of the 10 Einstein equations, 4 are conatraint equations and involve no dynamics. There is also a gauge freedom, in that the 4 coordinates, $x^{*}$, can be chomen abistrarily. So Einstein's equations can deacribe at mont 2 degrees of frondom (i.e. the 2 polarization states of a gravitational wave). By Birchorf's theorem ( we Weinberg (1972)), a apherical object cannot radiate gravitational waves (there is no preferred direction with which to asign directions of polarization). This menns that it abould be powible to find a coordinale system in which ihe metric is determined only by the 4 conatraint equation (metric instantaneously responding to matter and neutrinou), wince these are no gravitational degrees of freedom present.

\section{Isotropic Gauge}

\section{Einstein's Equations}

For a more complete discussion of Einstein's equations in the form vritten below, see Wilson(1979)). A sketch of the derivation follows.

In equation (22), use $\beta_{r}$ to force $A=B$ on each alice, and determine $\alpha$ by "maximal slicing", or $K_{i}^{i}=0$. (In equilibrium, and neglecting gravity, a soap film stretched across a closed wire loop, will assume a ahape such that $K_{i}^{i}=0$. In this case $K_{i j}$ is the extrinsic curvature of the sosp film (see Landou and Lifshitz (1959)).

It will be shown below that $K_{i}^{i}=0$ implies $\alpha=1+\phi$, in the limit of weak gravity, where $\phi=$ Newtonian gravitational potential.

In order to find the equation determining $\alpha$, use (5) in this appendix. Raise one index with $\Lambda^{i j}$, and take the trace of both aides of the equation. The following equation results:

$$
\frac{\partial K_{i}^{i}}{\partial t}=-D_{i} D^{i} \alpha+\alpha\left(K_{i j} K^{i j}+\frac{1}{2}(\rho H+S)\right)+\beta^{i} \frac{\partial K_{i}^{j}}{\partial x^{i}}
$$


Demanding $\frac{\partial x_{i}^{i}}{d i}=0$ and, $K_{i}^{i}=0$ in (23), the alicing condition for $a$ in found. In the gauge chosen, (23) becomea:

$$
\frac{1}{A^{3} r^{2}} \frac{\partial}{\partial r}\left(r^{2} A \frac{\partial \alpha}{\partial r}\right)=\alpha\left(\left(\rho+e_{m}\right)\left(u^{2}-\frac{1}{2}\right)+P_{m}\left(w^{2}+\frac{1}{2}\right)+\frac{3}{2}\left(K_{r}\right)^{2}+\rho_{r}\right)
$$

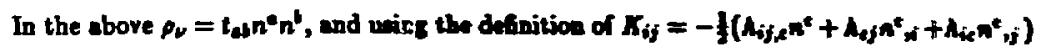
(wee MTW), it can be shown $K_{f}^{f}=K_{\phi}^{\phi}$. With thie reule, and $K_{j}^{j}=0$, the term in (23) $K_{i j} K^{i j}$, becomes ${ }_{2}^{3}\left(K_{r}^{\prime}\right)^{z}$ in $(24)$.

The condition $K_{i}^{j}=0$ cLn also be written down psing the definition of $\boldsymbol{K}_{j j}$ mentioned above:

$$
K_{i}^{i}=K_{r}^{r}+K_{i}^{i}+K_{i}^{t}=K_{r}^{r}+2 K_{i}^{p}=0
$$

implies:

$$
3\left(\frac{\partial A}{\partial t}-\beta^{r} \frac{\partial A}{\partial r}\right)-\frac{A}{r^{2}} \frac{\partial}{\partial r}\left(r^{2} \theta^{r}\right)=0
$$

Equation (25) will be further discusand after the constraint equations are written down. The Hamiltonian constraint equation (3a) becomes:

$$
\left.\frac{1}{r^{2}} \frac{\partial}{\partial r}\left(r^{2} \frac{\partial A^{\frac{1}{2}}}{\partial r}\right)=-\frac{A^{\frac{1}{2}}}{4}\left(\left(p+e_{m}+P_{m}\right) u^{2}-P_{m}+\rho_{\nu}+\frac{3}{4}: K_{r}^{r}\right)^{2}\right)
$$

The momentum constraint equation (3b) has only one nonzero component, and is writ ven as:

$$
\frac{1}{r^{3} A^{3}} \frac{\partial}{\partial r}\left(r^{3} A^{3} K_{r}^{r}\right)=S_{r}-j_{r}+\frac{\beta_{r}}{\alpha} p_{r}
$$

where:

$$
j_{r}=t_{r}^{e} n_{c}
$$

As mentioned proviously, $\beta^{r}$ is used to keep $A=B$ throughout the evol, ition. This means:

$$
\frac{\partial}{\partial t}\left(r^{2} \Lambda_{r r}\right)=\frac{\partial}{\partial t} \Lambda_{11}
$$

and:

$$
\frac{\partial}{\partial t}\left(\sin ^{2} \theta A_{y 0}\right)=\frac{\partial}{\partial t} \Lambda_{d \phi}
$$


must be true. Uning (6) in this appendix, a coordinate condition on $\rho^{*}$ can be found that guarantece $A=B$. It can be abown to be:

$$
\frac{\partial \rho r}{\partial r}-\frac{\rho_{r}^{r}}{r}-\frac{3}{2} \alpha K_{r}^{r}=0
$$

Now uning (28) and the equation from the set (6) for $4 x$, equation (25) can be reproduced. Tbis means the choice for is consistent with the choice $K_{i}^{i}=0$ for maximal alieing.

Four equations have been found to determine $K_{r}^{r}, \alpha, A, \beta r$ without using all of Einstein's equations. The complete set must be satisfied, and it will not be ahown, but the equations determining $K_{r}^{r}, \alpha, A, \beta^{r}$ in terms of spatial derivatives only, are consistent with the evolution equations determining $K_{r}^{r}$ and $A$ in terms of time derivatives. (For example, wolve (5) for $\frac{\partial K_{r}}{\partial t}$, and use (27) for $\frac{\partial x_{r}}{\partial r}$. If (27) is consistent with (5), then $\frac{f}{f t} \frac{\partial K_{r}}{\partial r}=\frac{\Delta x_{r}}{\partial t}$ using (27) in the left hand side, and (5) in the right hand side. To show the equality, $\left(T_{*}^{+}+t_{k}\right), \alpha=0$ is also needed)

The equations determining the metric variables are rewritten below:

momentum constraint

$$
K_{r}^{r}=\frac{1}{r^{3} A^{3}} \int_{0}^{r} r^{3} A^{3}\left(S_{r}-j_{r}+\frac{\beta_{r}}{\alpha} p_{\nu}\right)
$$

coordinate condition

$$
\beta^{r}=-\frac{3}{2} r \int_{r}^{\infty} \frac{\alpha K_{r}^{r}}{r} d r
$$

\section{Hamiltonian constraint}

$$
\frac{1}{r^{2}} \frac{\partial}{\partial r}\left(r^{2} \frac{\partial A^{\frac{1}{2}}}{\partial r}\right)=-\frac{A^{\frac{k}{2}}}{4}\left(\left(\rho+e_{m}+P_{m}\right) u^{2}-P_{m}+\rho_{\nu}+\frac{3}{4}\left(K_{r}^{r}\right)^{2}\right)
$$

marimal slicing

$$
\frac{1}{A^{3} r^{2}} \frac{\partial}{\partial r}\left(r^{2} \frac{\partial \alpha}{\partial \alpha}\right)=\alpha\left(\left(\rho+e_{m}\right)\left(u^{2}-\frac{1}{2}\right)+P_{m}\left(u^{2}+\frac{1}{2}\right)+\frac{3}{2}\left(K_{r}^{r}\right)^{2}+\rho_{\nu}\right)
$$


Thus the isotropic gauge, along with maximal alicing, allow the metric variable to be found by solving ordiuary differential equation, explicity demonatratiag the leck of gravity waves in a spherical geomstry.

Finally in the limit of weak gravity, where the wource term in (24) and and (20) are dominated by the rest man matter density, it can be wen by direct wbatitution that $\alpha=1+\phi$ and $A=1-\phi$, where $\phi$ in the Newtonien gravitational potential determined from Poiswon's equation:

$$
\frac{1}{r^{2}} \frac{\partial}{\partial r}\left(r^{2} \phi\right)=4 \pi G_{\rho}=\frac{\rho}{2}
$$

Neutrino Boltzmann Equation

Next, the neutrino Boltzmann equation, and the comotruetion of the neutrino atrom energ: tensor will be considered. Only one opecien will be done an an example; bowerer, 6 Boltzmann equations must actually be found, and $t_{a t}$ really contains contributions from 6 apecies.

Now the neutrino momentum can be written in terme of the tetred unit rectors (thoese unit vectors that are locally Minkowskian in the apecelike olice):

$$
\bar{p}=p^{0} z_{0}+p^{1} z_{1}+p^{2} z_{2}+p^{9} z_{3}
$$

where:

$$
\begin{gathered}
\left(z_{0}\right)^{*}=n^{*}=\text { normal to opacelike aliee } \\
\left(z_{1}\right)^{r}=\frac{1}{A} ;\left(z_{1}\right)^{*}=0, a \neq r \\
\left(z_{3}\right)^{*}=\frac{1}{A r} ;\left(z_{2}\right)^{*}=0, a \neq 0 \\
\left(z_{3}\right)^{*}=\frac{1}{\text { Arain }} ;\left(z_{3}\right)^{*}=0, a \neq \phi
\end{gathered}
$$

snd:

$$
\begin{gathered}
p^{0}=E \\
p^{1}=E \cos \eta=E \mu \\
p^{2}=E \sin \eta \cos \xi=E\left(1-\mu^{2}\right)^{\frac{1}{2}} \cos \xi \\
p^{3}=E \sin \eta \sin \xi=E\left(1-\mu^{2}\right)^{\frac{1}{2}} \sin \xi
\end{gathered}
$$


Figure 3 defines the angles uned in the above. Following wileon (unpubliabed notec), the Boltzmann equation takes on a relatively simple form if, inatead of $E$ (the energy mearured by the Eulerian obeerver), $W=E A$ in und. If thi in $\infty$, then:

$$
\begin{gathered}
p^{t}=\frac{W}{\alpha A} \\
p^{r}=\frac{W}{A}\left(\frac{\mu}{A}-\frac{\rho^{r}}{\alpha}\right) \\
p^{\prime}=\frac{W}{A^{2}} \frac{\left(\Sigma-\mu^{2}\right)^{\frac{1}{3}}}{r} \cos \xi \\
p^{\prime}=\frac{W}{a^{2}} \frac{\left(1-\mu^{2}\right)^{\frac{1}{2}}}{r \sin } \sin \xi
\end{gathered}
$$

are the contravariant components of $p^{\infty}$, while the covariant components are:

$$
\begin{gathered}
p_{t}=\beta^{+} \mu W-\frac{W}{A} \alpha \\
p_{r}=\mu W \\
p_{l}=r\left(1-\mu^{2}\right)^{\frac{1}{2} W} \cos \xi \\
p_{\phi}=r\left(1-\mu^{2}\right)^{\frac{1}{2}} W \sin \xi \sin \varphi
\end{gathered}
$$

With this momentum parameterization, $p_{r}=\mu W$, the radial component of the covariant momentum, takes on a simple form.

Also introduce $H=r^{2} W^{2} f$, which is a kind of geutrino number luminosity, and the Boltzmann equation in conservation form becomen:

$$
\begin{gathered}
\frac{\partial \hat{H}}{\partial t}+\frac{\partial}{\partial r}\left(\hat{H}\left(\frac{\alpha \mu}{A}-\beta^{r}\right)\right)+\frac{\partial}{\partial W}\left(\tilde{H W}\left(\frac{\left(1-\mu^{2}\right) \beta^{r}}{r}-\mu\left(\frac{\partial}{\partial r}\left(\frac{\alpha}{A}\right)-\mu \frac{\partial \beta^{r}}{\partial r}\right)\right)\right) \\
+\frac{\partial}{\partial \mu}\left(\tilde{H}\left(\left(\frac{\alpha}{A}-\mu \beta^{r}\right) \frac{1-\mu^{2}}{r}-\left(1-\mu^{2}\right)\left(\frac{\partial}{\partial r}\left(\frac{\alpha}{A}\right)-\mu \frac{\partial \beta^{r}}{\partial r}\right)\right)\right) \\
=\alpha r^{2} W^{2} \frac{A}{W} \text { (collioions terms) }
\end{gathered}
$$

where:

$$
\text { collision terms }=\kappa \rho(I-f)
$$

Lindquist defines $\boldsymbol{k}$ so that it is an invariant or scalar quantity. This means knowledge of $x$ in the fluid frame allows the calculation in any frame. Lindquist finds: 


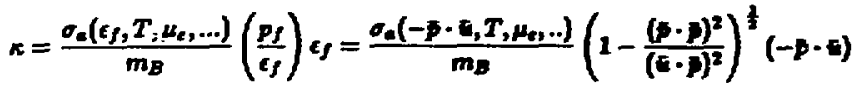

where the rightmont equality ohowa $x$ is a $x a l a r$ quanity. pg is the magnitude of the neutrino momentum as mearured by the fluid oboerver $\left(p_{f}=c\right.$, for a macolem particle) and all dot products are 4 vector dot products. The abeorbtive opmcity is denoted by $\sigma_{a}\left(\epsilon_{S}, T, \mu_{e}, ..\right) / m_{B}$. It depends in general os the neutrino energy, matter temperature, electron chemical potential, matter composition, etc. $x$ is thus meen to be (opacity)(momentum). The momentum factor comes from the fact that (21), the Boltzmann equation can be written more elegantly with a factor of momentum multiplying both sides of tbe equation.

As an example to illustrate the form of the collinion term, consider pure abmorbtion and emission processes. The energy of a neutrino in the fluid frame (as meanud by the fluid observer), is found from:

$$
\epsilon_{p}=-\mathbf{p} \cdot \boldsymbol{t}
$$

Using (30), the right hand side of (29) becomes:

$$
\alpha A \tau^{2} W \kappa \rho(I-f)=\frac{\rho \sigma_{a}}{m_{B}} \alpha u\left(1-\mu \frac{\beta^{r}+v^{*}}{\alpha} A\right)(B-H)
$$

where $B$ is an emission function. Assuming the emitting particles are in local thermodynamic equilibrium:

$$
I=\frac{1}{\exp \left(\frac{c_{f}-\mu_{v}}{k_{g} T}\right)+1}
$$

and:

$$
B=\frac{r^{2} W^{2}}{\exp \left(\frac{\epsilon_{f}-\mu_{\nu}}{k_{B} T}\right)+1}
$$

Also used in (31) is the fact:

$$
\epsilon_{f}=-\bar{p} \cdot \bar{u}=\frac{u W}{A}\left(1-\mu \frac{\beta^{r}+v^{r}}{a} A\right)
$$

The conservative form of Boltzmann's equation is useful for numerical computation as it facilitates construction of a finite difference form of the equation (see Roache (1972)).

To discuss limiting cases of the Boltzmann equation, it is more useful to return to $f$ as an independent variable. In terms of $f,(29)$ becomes: 


$$
\begin{gathered}
\frac{1}{\alpha} \frac{\partial f}{\partial t}+\frac{1}{A}\left(\mu-\frac{\beta^{r} A}{\alpha}\right) \frac{\partial f}{\partial r}+W \frac{\partial f}{\partial W}\left(\frac{\left(1-\mu^{2}\right) \beta^{r}}{\alpha r}-\mu\left(\frac{1}{\alpha} \frac{\partial}{\partial r}\left(\frac{\alpha}{A}\right)-\frac{\mu \beta^{r}}{\partial r}\right)\right) \\
+\left(1-\mu^{2}\right) \frac{\partial f}{\partial \mu}\left(\left(1-\frac{\mu \beta^{r} A}{\alpha}\right) \frac{1}{A r}-\left(\frac{1}{\alpha} \frac{\partial}{\partial r}\left(\frac{\alpha}{A}\right)-\frac{\mu^{2}}{\alpha} \frac{\partial \beta^{r}}{\partial r}\right)\right) \\
=\frac{A}{W} \kappa \rho(I-\rho)
\end{gathered}
$$

In the limit $\alpha=A=1$, and $\beta^{*}=0$, (32) becomes:

$$
\frac{\partial f}{\partial t}+\mu \frac{\partial f}{\partial r}+\left(1-\mu^{2}\right) \frac{\partial f}{\partial \mu}=\frac{\partial f}{\partial t}+\hat{p} \cdot \nabla f=\text { collisions }
$$

This is the familiar radiation traneport equation from photon radiation transport.

The terms involving $\alpha, A$, and $\beta^{r}$ contain relativistic effects. As mentioned before $\alpha=$ $1+\phi$ and $A=1-\phi$ in the limit of weak gravity. Setting $\alpha=1+\phi, A=1-\phi, \beta^{r}=0$, and assuming the atatic limit, (32) becomes:

$$
\begin{gathered}
\frac{\mu}{A} \frac{\partial f}{\partial r}+\frac{\left(1-\mu^{2}\right)}{A r} \frac{\partial f}{\partial \mu}-\frac{\mu}{\alpha} W \frac{\partial f}{\partial W}\left(2 \frac{\partial \phi}{\partial r}\right)-\frac{\left(1-\mu^{2}\right)}{\alpha} \frac{\partial f}{\partial \mu}\left(2 \frac{\partial \phi}{\partial r}\right) \\
=\text { collisions }
\end{gathered}
$$

The third term in the above appears due to the redohift of the neutrino energy, and the fourth term takes into account the fact that the nentrino trajectory deviates from a straight line path in the presense of a strong gravitational field. The above can be written in a more suggestive form (neglecting $\phi$ compared to unity) as:

$$
c \hat{p} \cdot \nabla f-\frac{2 p}{c^{2}} \nabla \phi \cdot \frac{\partial f}{\partial \vec{p}}=\text { collision terms }
$$

where dot products are ordinary three vector dot products, $p=W=$ neutrino energy, and $c$ (i.e. speed of light), has been reintroduced. This looks like the nonrelativistic Boltzmann equation:

$$
\hat{c \hat{p}} \cdot \nabla f+\vec{F} \cdot \frac{\partial f}{\partial \bar{p}}=\text { colliaions }
$$

with $\vec{F}=\frac{2 p}{c^{2}}(-\nabla \phi)$ as the force on a neutrino due to gravity.

Fivally the terms in the left hand aide of (32) involving $\boldsymbol{\beta}^{r}$ come from the fact that $\bar{E}$ and $\mu$ are defined in the static oberaver frame and $-\frac{p}{a}$ is a boost velocity from the coordinate 
observer frame to the Eulerian frame. In the limit $\alpha=A=1$, and $-F$ in the velocity of the Lorentz boost to the frame where the enersy and angle are meacured, the recult of Mihala (1978) is found. The neutrino utrew energy tensor $t_{\alpha b}$ will now be conatructed:

$$
t^{\omega}=\int s^{c} s d P
$$

Lindquist (1966) finds the following expremion for the invariant 3 momentum rolume:

$$
d P=\sqrt{ }-\operatorname{Det} \rho_{\alpha} \frac{d p^{r} d p^{d} d p^{d}}{-p_{t}}
$$

By doing a change of variables, $d P$ can be exprewed as:

$$
d P=E d E d \mu d \xi=\frac{W d W}{A^{2}} d \mu d \xi
$$

Now defining new variables:

$$
\begin{gathered}
\tilde{E}=\int f E^{s} d E d \mu d \xi=\text { neutrino energy density } \\
\tilde{G}=\int f E^{s} \mu d E d \mu d \xi=\text { neutrino energy flux } \\
\tilde{P}=\int f E^{s} \mu^{2} d E d \mu d \xi=\text { neutrino radial pressure }
\end{gathered}
$$

the following is found for $t_{a}^{b}$ :

$$
t_{a}^{b}=\left(\begin{array}{cccc}
-\tilde{e}+\frac{e^{r} \Lambda}{\alpha} \tilde{G} & \beta^{r}(\tilde{e}+\tilde{P})-\frac{\Delta}{\alpha} \tilde{G}\left(\left(\beta^{r}\right)^{2}+\frac{a^{2}}{A^{2}}\right) & 0 & 0 \\
\tilde{G} \frac{A}{\alpha} & \tilde{P}-\frac{\theta^{r} A}{\alpha} \tilde{G} & 0 & 0 \\
0 & 0 & \frac{j-\tilde{P}}{2} & 0 \\
0 & 0 & 0 & \frac{i-\tilde{P}}{2}
\end{array}\right)
$$

where $a$ denoted the row index and $b$ the column index. Note that $t_{a}^{a}=0$, since it describes a massless particle.

From $t^{a b}$, the neutrino contributions to the source terms in the gravitational equations (24),(26),(27),(28) can be found from:

$$
\begin{gathered}
p_{\nu}=t^{a b} n_{a} n_{b} \\
j_{r}=t_{r}^{c} n_{c}
\end{gathered}
$$

For the energy equation (14) and, Euler's equation(16), $t_{a ; k}^{b}$ will be needed. This could be formed using: 


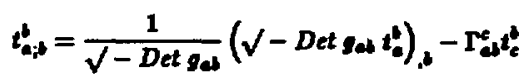

However, an alternate way is to use the transport equation:

$$
+\infty
$$

where:

$$
e^{*}=\int d P p^{*} x p(I-f)
$$

This result follows by integrating (21) over $d P$ after multiplying both wides of the equation by $p^{a}$ (see Lindquist(1966)).

For the spherical problem, the components $q_{r}$ and $q_{t}$ are:

$$
\begin{gathered}
g_{t}=-\int E^{S} d E d \mu d \xi\left(i-\frac{\beta_{r} A}{\alpha} \mu\right) k \rho(I-f) \\
q_{r}=\frac{A}{\alpha} \int E^{2} d E \mu d \mu d \xi k_{p}(I-f)
\end{gathered}
$$

where $k=\frac{\sigma}{m_{B}}$ is the opacity measured in the fluid frame.

For the lepton conservation equation, $N_{j}$ is needed. This can also be found from the transport equation (21). Multiplying both sides by $d P$ and integrating results in:

$$
N_{; a}^{a}=\int d P_{x \rho}(I-f)=\int E^{2} d E d \mu d \xi k \rho(I-f)
$$

\section{Summary}

Below is a summary of the complete set of evolution equations in the isotropic gauge. For simplicity, only one type of ueutrino is assumed.

metric

$$
d s^{2}=-\left(\alpha^{2}-\beta_{r} \beta^{r}\right) d t^{2}+2 \beta_{r} d r d t+A^{2} d r^{2}+A^{2} r^{2} d \Omega^{2}
$$




$$
\begin{gathered}
\text { gravity } \\
K_{r}^{r}=\frac{1}{r^{3} A^{3}} \int_{0}^{r} r^{3} A^{3}\left(S_{r}-j_{r}+\frac{\beta_{r}}{a} \rho_{\nu}\right) \\
\beta^{r}=-\frac{3}{2} r \int_{r}^{\infty} \frac{\alpha K_{r}^{r}}{r} d r \\
\frac{1}{r^{2}} \frac{\partial}{\partial r}\left(r^{2} \frac{\partial A^{\frac{1}{2}}}{\partial r}\right)=-\frac{A^{\frac{k}{2}}}{4}\left(\left(\rho+e_{m}+P_{m}\right) u^{2}-P_{m}+\rho_{\nu}+\frac{3}{4}\left(K_{r}^{r}\right)^{2}\right) \\
\frac{1}{A^{3} r^{2}} \frac{\partial}{\partial r}\left(r^{2} A \frac{\partial \alpha}{\partial r}\right)=\alpha\left(\left(\rho+e_{m}\right)\left(u^{2}-\frac{1}{2}\right)+P_{m}\left(u^{2}+\frac{1}{2}\right)+\frac{3}{2}\left(K_{r}^{r}\right)^{2}+\rho_{\nu}\right)
\end{gathered}
$$

neutrino Boitzmann equation

$$
\begin{gathered}
\frac{\partial \bar{H}}{\partial t}+\frac{\partial}{\partial r}\left(\bar{H}\left(\frac{\alpha \mu}{A}-\beta^{r}\right)\right)+\frac{\partial}{\partial W}\left(\bar{H} W\left(\frac{\left(1-\mu^{2}\right) \beta^{r}}{r}-\mu\left(\frac{\partial}{\partial r}\left(\frac{\alpha}{A}\right)-\mu \frac{\partial \beta^{r}}{\partial r}\right)\right)\right) \\
+\frac{\partial}{\partial \mu}\left(\bar{H}\left(\left(\frac{\alpha}{A}-\mu \beta^{r}\right) \frac{1-\mu^{2}}{r}-\left(1-\mu^{2}\right)\left(\frac{\partial}{\partial r}\left(\frac{\alpha}{A}\right)-\mu \frac{\partial \beta^{r}}{\partial r}\right)\right)\right) \\
=\alpha r^{2} W^{2} \frac{A}{W} \text { (collisions terms) }
\end{gathered}
$$

baryon conservation

$$
\frac{\partial D}{\partial t}+D \frac{\partial \ln \Upsilon^{\frac{1}{2}}}{\partial t}+\frac{1}{\Upsilon^{\frac{1}{2}}} \frac{\partial}{\partial r}\left(\Upsilon^{\frac{1}{2}} v^{r} D\right)+0
$$

energy conservation

$$
\begin{gathered}
\frac{\partial E}{\partial t}+E \frac{\partial \ln \Upsilon^{\frac{1}{2}}}{\partial t}+\frac{1}{\Upsilon^{\frac{1}{2}}} \frac{\partial}{\partial r}\left(E v^{r} \Upsilon^{\frac{1}{2}}\right)+\frac{P_{m}}{\Upsilon^{\frac{1}{2}}}\left(\frac{\partial\left(u \Upsilon^{\frac{1}{2}}\right)}{\partial t}+\frac{\partial}{\partial r}\left(u v^{r} \Upsilon^{\frac{1}{2}}\right)\right) \\
=u\left(q_{t}+v^{r} q_{r}\right)
\end{gathered}
$$

\section{Euler's equation}

$$
\begin{aligned}
& \frac{\partial S_{r}}{\partial t}+S_{r} \frac{\partial \ln \Upsilon^{\frac{1}{2}}}{\partial t}+\frac{1}{r^{\frac{1}{2}}} \frac{\partial}{\partial r}\left(S_{r} v^{r} \Upsilon^{\frac{1}{2}}\right)+\alpha \frac{\partial P_{m}}{\partial r} \\
& +\alpha S^{t} \frac{\partial \alpha}{\partial r}-S_{r} \frac{\partial \beta^{r}}{\partial r}+\frac{1}{2} \frac{S_{r}^{2}}{S_{t}} \frac{\partial}{\partial r}\left(\frac{1}{A^{2}}\right)=-\alpha g_{r}
\end{aligned}
$$




\section{lepton number conservation}

$$
\frac{D}{m_{B}}\left(\frac{\partial \bar{Z}}{\partial t}+v^{r} \frac{\partial \bar{Z}}{\partial r}\right)=-\int E^{2} d E d \mu d \xi k \rho(I-f)
$$

$$
\begin{gathered}
\text { charge conservation } \\
\frac{\partial \bar{Z}}{\partial t}+v^{r} \frac{\partial \bar{Z}}{\partial r}=\frac{\partial Z}{\partial t}+v^{r} \frac{\partial Z}{\partial r}
\end{gathered}
$$

from $u_{a} u^{a}=-1, S_{\alpha} S^{a}=-\left(\rho+e_{m}+P_{m}\right)^{2} u^{2}, n_{s} S^{*}=-\left(\rho+e_{m}+P_{m}\right) u^{2}$ and $u=-n^{a} u_{s}$, expressions for $S^{t}, u$, and $v^{r}$ can be found:

$$
\begin{gathered}
S^{i}=\frac{1}{\alpha}\left(\left(D+E+P_{m} U\right)^{2}+\frac{S_{r}^{2}}{A^{2}}\right)^{\frac{1}{2}} \\
u=\frac{\alpha S^{t}}{D+E+P_{m} u} \\
v^{r}=\frac{u^{r}}{u^{t}}=\frac{S_{r}}{A^{2} S^{t}}-\beta^{r}
\end{gathered}
$$

A second choice of gauge is now presented.

\section{Schwarzschild Gauge}

In this section, a much briefer discussion of the appropiate equations will be given. The complete set has already been written by Wilson(1971).

To find the metric in this gauge, set $B=1$ and $\beta^{r}=0$ in (22). Changing to the metric signature used by Wilson (i.e. $(+,-,-,-\lambda)$ and to notation more in line with his notation, the metric becomes:

$$
d s^{2}=a^{2} d t^{2}-b^{2} d m^{2}-R^{2} d \Omega^{2}
$$

The condition determining $a$ is not maximal slicing, but that $u^{t}=\frac{1}{a}, u^{r}=0$. The coordinate system is thus Lagrangian. 
The total atress energy tensor (note $\left.T_{a b}=\left(b+e_{m}+P_{m}\right) u_{a} v_{b}-P_{m} g_{a b}\right)$ is:

$$
T_{c}^{d}+t_{c}^{d}=\left(\begin{array}{cccc}
-P_{t o t} & 0 & 0 & -\frac{t}{G} \\
0 & -P_{10 t}-H & 0 & 0 \\
0 & 0 & -P_{t g d}-H & 0 \\
t \tilde{G} & 0 & 0 & +e_{t a t}
\end{array}\right)
$$

where $c$ denotes the row index, $d$ the column index in the above matrix representation, and:

$$
H=\frac{1}{2}(\tilde{e}-3 \tilde{P})
$$

where $\tilde{e}, \tilde{G}, \tilde{P}$ are neutrino energy, lux, and pressure, and $P_{l a t}=\tilde{P}+P_{m}, e_{l e c}=\tilde{e}+e_{m}$.

The equation for baryon number conservation, $\left(\rho u^{*}\right)_{; \epsilon}=0$ results in: .

$$
\frac{\partial}{\partial t}\left(b R^{2} \rho\right)=0
$$

This is integrated to give:

$$
b=\frac{1}{4 \pi R^{2} p}
$$

where the integration constant is chosen to be $\frac{1}{4 \pi}$. With this choice, $m$ is the total rest mass enclosed by a sphere with radius $m$, since:

$$
m=\int \rho d V=\int_{0}^{m} \rho\left(4 \pi R^{2} b\right) d m=\int_{0}^{m} d m
$$

The equation for $a$ is determined from the conservation of stress energy:

$$
\left(T_{m}^{e}+t_{m ; i c}^{c}\right.
$$

This equation becomes (after some simplification):

$$
a=\exp \int_{R}^{\infty}\left(\left(\frac{\partial P_{t o t}}{\partial m}+\frac{2 H}{R} \frac{\partial R}{\partial m}+\frac{\partial}{\partial t}\left(\frac{\tilde{G} b}{a}\right)\right) \frac{d m}{\left(p+e_{t o t}+P_{t o t}\right)}\right)
$$

The energy conservation equation, $u^{c}\left(T_{i}^{d}+t_{c}^{d}\right)_{; d}=0$ becomes:

$$
\frac{1}{a} \frac{\partial\left(e_{m} / \rho\right)}{\partial t}+\frac{P_{m}}{a} \frac{\partial}{\partial t}\left(\frac{1}{\rho}\right)=-\frac{u^{c}}{\rho} t_{t ; d}^{d}
$$

The Hamiltonian constraint ( $a$ second order equation in $m$ and $t$ ) becomes two first order equations: 


$$
\begin{aligned}
& \frac{1}{b} \frac{\partial R}{\partial m}=\left(1+U^{2}-\frac{2 M}{R}\right)^{\frac{1}{2}} \equiv \Gamma \\
& \frac{\partial M}{\partial R}=4 \pi\left(\rho+e_{t c t}+\frac{\tilde{G} U}{\Gamma}\right) R^{2}
\end{aligned}
$$

where:

$$
U=K_{n}=\frac{1}{c} \frac{\partial R}{\partial t} .
$$

and the equation for $\frac{\partial M}{\partial R}$ can be integrated to give:

$$
M=4 \pi \int_{0}^{R}\left(\rho+e_{\mathrm{tot}}+\frac{\tilde{G} U}{\Gamma}\right) R^{2} d R
$$

$M$ is seen to be a mass energy (ircluding gravitational effects), and $U$ is a component of the extrinsic curvature tensor. It can be shown (bee May and White(1967)) that $U$ is also the radial component of the fluid 4 velocity as measure 1 by an Euierian observer. In the limit gravity is neglected, $\Gamma=\frac{1}{\sqrt{1-v^{2}}}$. Thus $\Gamma$ is a generalization of a special relativistic gamma (i.e. $U=\Gamma v$ ).

In this gauge, some of Einstein's evolution equations are used, instead of only using the constraint equations, as in the isotropic gauge. The resulting choice is motivated by ease of interpretation of the equations. (Again, in the case where symmetries are present, Einstein's equations are overdetermined, in that a smaller subset implies the whole set.)

The evolution equation for $K_{10}$ results in:

$$
\begin{aligned}
& \frac{1}{a} \frac{\partial K_{b \theta}}{\partial t}=\frac{1}{a} \frac{\partial U}{\partial t}=-\frac{4 \pi R^{2} \Gamma}{\left(1+\frac{P_{10 c t}+P_{c o t}}{f}\right)}\left(\frac{\partial P_{t o t}}{\partial m}+\frac{2 H}{R} \frac{\partial R}{\partial m}+\frac{\partial}{\partial t}\left(\frac{\tilde{G} b}{a}\right)\right) \\
& -\frac{G M}{R^{2}}\left(1+\frac{4 \pi P_{l o t} R^{3}}{M}\right)
\end{aligned}
$$

In the Newtonian limit, this equation looks like Force $=$ (mass)(acceleration), even though it is one of Einstein's equations.

A final equation is needed, and $G^{m m}=T^{m m}+t^{m m}$ is chosen. This equation becomes:

$$
\frac{1}{a} \frac{\partial}{\partial t} \ln \left(\rho R^{2}\right)+\frac{\partial U}{\partial m} / \frac{\partial R}{\partial m}=0
$$

In the Newtonian limit, this is the mass conservation equation, again giving a hydrodynamic equation from one of Einstein's equations. 
For the neutrino Boltzmann equation, introduce $Q$ such that $D=\frac{Q}{G}$ where $D=\epsilon_{j}=$ $+\bar{p} \cdot \bar{u} . Q$ is the energy sn observer at irfinity would measure for a beutrino emitted with energy $E$, which had escaped with no further interactions. (Note that with this metric signature $u^{a} u_{0}=+1$, and $\epsilon_{g}=+p \cdot \bar{u}$.)

Also introduce a new dependent variable, $F$, wuch that:

$$
\tilde{\varepsilon}=\int f E^{s} d E d \mu d \xi=\int F d E d \mu=\int F \frac{2 Q}{a} d \mu
$$

so:

$$
F=2 \pi f E^{*}
$$

The equation for neutrino transport becomes:

$$
\begin{gathered}
\frac{1}{a} \frac{\partial F}{\partial t}=-\frac{\mu \Gamma}{a R^{2}} \frac{\partial}{\partial R}\left(a R^{2} F\right)-\Gamma\left(\frac{1}{R}-\frac{\partial}{\partial R} \ln a\right)\left(\frac{\partial}{\partial \mu}\left(F\left(1-\mu^{2}\right)\right)\right) \\
+\frac{F}{a \rho} \frac{\partial \rho}{\partial t}+R \frac{\partial}{\partial R}\left(\frac{U}{R}\right)\left(\frac{\partial}{\partial \mu}\left(\mu\left(1-\mu^{2}\right) F\right)+\mu^{2} Q \frac{\partial F}{\partial Q}\right) \\
+\frac{Q}{a} \frac{\partial F}{\partial Q}\left(\frac{\partial}{\partial t} \ln \frac{R}{a}\right)+k \rho(B-F)
\end{gathered}
$$

If simple absorbtion and emission processes are involved, then:

$$
B=\frac{2 \pi \varepsilon_{f}^{3}}{\exp \left(\frac{\epsilon_{1}-\mu_{\mu}}{t_{B} T}\right)+1}
$$

where the emitting particles are assumed in thermodynamic equilibrium.

The equation for energy conservation becomes:

$$
\frac{1}{a} \frac{\partial\left(e_{m} / \rho\right)}{\partial t}+\frac{P_{m}}{a} \frac{\partial}{\partial t}\left(\frac{1}{\rho}\right)=-\int k(B-F) d \mu \frac{d Q}{a}
$$

The equaticn for lepton number conservation is found using (17):

$$
\frac{1}{a} \frac{\rho}{m_{B}} \frac{\partial \bar{Z}}{\partial t}=-\int(B-F) \frac{d Q}{Q} d \mu
$$

And the equation for charge conservation (19) becomes:

$$
\frac{1}{a} \frac{\partial \bar{Z}}{\partial t}=\frac{1}{a} \frac{\partial Z}{\partial t}
$$

Summary

A summary of the supernova equations in the Schwarzschild gauge follows: 
metric

$$
d t^{2}=a^{2} d t^{2}-b^{2} d m^{2}-R^{2} d R^{2}
$$

$$
\begin{aligned}
& M=4 \pi \int_{0}^{R}\left(\rho+e_{t o t}+\frac{\tilde{G} U}{\Gamma}\right) R^{2} d R \\
& \frac{1}{b} \frac{\partial R}{\partial m}=\left(1+U^{2}-\frac{2 M}{R}\right)^{\frac{1}{2}} \equiv \Gamma
\end{aligned}
$$

$$
\begin{aligned}
& \frac{1}{a} \frac{\partial K_{\theta U}}{\partial t}=\frac{1}{a} \frac{\partial U}{\partial t}=-\frac{1 \pi R^{2} \Gamma}{\left(1+\frac{\left.2 \sin +P_{a t}\right)}{p}\right.}\left(\frac{\partial P_{\mathrm{lot}}}{\partial m}+\frac{2 H}{R} \frac{\partial R}{\partial m}+\frac{1}{c^{2}} \frac{\partial}{\partial t}\left(\frac{\bar{G} b}{a}\right)\right) \\
& -\frac{G M}{R^{2}}\left(1+\frac{1 \pi P_{\text {lot }} R^{5}}{M}\right) \\
& U=\frac{1}{a} \frac{\partial R}{\partial t} \\
& \frac{1}{a} \frac{i}{\partial t} \ln \left(\rho R^{2}\right)+\frac{\partial U}{\partial m} / \frac{\partial R}{\partial m}=0
\end{aligned}
$$

\section{neutrino Boltzmann equation}

$$
\begin{gathered}
\frac{1}{a} \frac{\partial F}{\partial t}=-\frac{\mu \Gamma}{a R^{2}} \frac{\partial}{\partial R}\left(a R^{2} F\right)-\Gamma\left(\frac{1}{R}-\frac{\partial}{\partial R} \ln a\right)\left(\frac{\partial}{\partial \mu}\left(F\left(1-\mu^{2}\right)\right)\right) \\
+\frac{F}{a \rho} \frac{\partial \rho}{\partial t}+R \frac{\partial}{\partial R}\left(\frac{U}{R}\right)\left(\frac{\partial}{\partial \mu}\left(\mu\left(1-\mu^{2}\right) F\right)+\mu^{2} Q \frac{\partial F}{\partial Q}\right) \\
+\frac{Q}{a} \frac{\partial F}{\partial Q}\left(\frac{\partial}{\partial t} \ln \frac{R}{a}\right)+k \rho(B-F)
\end{gathered}
$$

baryon conservation

$$
b=\frac{1}{1 \pi R^{2} \rho}
$$


otrees energy conervation

$$
\begin{gathered}
a=\exp \int_{R}^{\infty}\left(\left(\frac{\partial P_{l m}}{\partial m}+\frac{2 B}{R} \frac{\partial R}{\partial m}+\frac{\partial}{\partial t}\left(\frac{\tilde{G} b}{a}\right)\right) \frac{d m}{\left(\rho+l_{t \omega t}+F_{L c}\right)}\right) \\
\frac{1}{a} \frac{\partial\left(e_{m} / \rho\right)}{\partial t}+\frac{P_{m}}{a} \frac{\partial}{\partial t}\left(\frac{1}{\rho}\right)=-\int k(B-F) d L \frac{d Q}{a}
\end{gathered}
$$

Iepton number conservation

$$
\frac{1}{a} \frac{\rho}{m_{B}} \frac{\partial \bar{Z}}{\partial t}=-\int(B-F) \frac{d Q}{Q} d \mu
$$

charge conservation

$$
\frac{1}{a} \frac{\partial \bar{Z}}{\partial t}=\frac{1}{a} \frac{\partial Z}{\partial t}
$$


Notation uned in Appendix A (partia) linting)

$G_{a b}=$ Einstein tensor

$T_{a b}=$ matter stress energy tensor

$t_{a b}=$ neutrino wtress energy tencor

$g_{a b}=1$ metric

$n^{\circ}=$ unit vector to spacelike alice

$\boldsymbol{K}_{i j}=$ extrinsic curvature

$\Lambda_{i j}=3$ metric

${ }^{1} R_{b e d}^{a}=1$ dimensional Riemann tensor

$D_{i}=$ covariant derivative compatible with $\Lambda_{i j}$

${ }^{3} R=$ Ricci acalar comparible with $A_{i j}$

$\rho_{H}=n^{a} n^{b}\left(T_{a b}+t_{a b}\right)$

$J_{i}=-\Lambda_{i}^{a} n^{b}\left(T_{a b}+t_{a b}\right)$

$\beta^{a}=$ shift vector

$\alpha=$ lapse function

$t^{a}=\alpha n^{a}+\beta^{a}$

${ }^{3} R_{i j}=$ Ricci tensor compatible with $\Lambda_{i j}$

$S_{i j}=\Lambda_{i}^{a} \Lambda_{j}^{b}\left(T_{a b}+t_{a b}\right)$

$s=s_{i}^{i}$

$n_{B}=$ baryon density measured in rest frame

$u^{\circ}=$ matter 4 velocity

$p=$ matter rest mass density

$D=$ density measured by Eulerian observer

$v^{i}=3$ velocity of fluid

$\boldsymbol{u}=$ generalization of special relativistic gamma

$T^{\frac{1}{2}}=\sqrt{ } \operatorname{Det}\left(\Lambda_{i j}\right)$

$f=$ neutrino distribution funciion

$d P=3$ momentum volume element

$p^{\alpha}=$ neutrino 4 momentum

$N^{a}=$ neulrino flux 4 vector

$\rho_{\nu}=t_{a b} n^{a} n^{b}$

$j_{r}=t_{\boldsymbol{r}}^{c} n_{c}$ 
$\epsilon_{f}=$ neutrino energy measured by fluid oberver

$q^{\alpha}=1 ; b$

$k=$ non-relativistic opacity

$a=$ lapse function

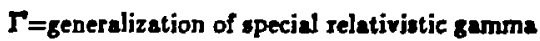




\section{Appendix A Reference}

Landau, L.D. and Liftshitz. E. M. 1959, "Fluid Mechanica"

(Pergamon Press:London) Chapter 7.

Lindquist, R.W. 1966, Ann. Phyz. (USA), v37, 487.

Mibalas, D. 1978, "Stellar Atmonpheres",(W.H. Freeman and Company:San Franciaco)

Chapter 14, Equation 130.

Misner, C.W., Thorne, K.S., and Wheeler, J.A. 1973, "Gravitation"

(W.H. Freeman and Company:San Francisco)

Particle Properties Data 1981, "Rev. Mod. Phys.”, $v 56$.

Roach, P.J. 1972, "Computational Fluid Dynarnics",

(Hermosa Publishers:Albuquerque, NM)

Shapiro, S.L. and Teukolsky, S.A. 1980, Ap. J. v235, 199.

Weinberg, S. 1972, "Gravitation and Cosmology",

(John Wiley and Sons, Inc.:New York)

Wilson, J.R. 1979 in "Sources of Gravitational Radiation", ed. L. Smarr

(Cambridge University Press:Cambridge) p.423

York, J.W. 1979 in "Sourcess of Gravitational Radiation", ed. L. Smarr

(Cambridge University Press:Cambridge) p. 83 


\section{Appendix A Figure Captions}

Fig. 1 Tlustration of the definition of the vector field $t^{*}$.

Fig. 2 Figure used in finding the fluid 3 velocity $v_{E}^{i}$, or the three velocity as measured by the Eulerian observer.

Fig. 3 Definition of the various angles used in neutrino transport. 


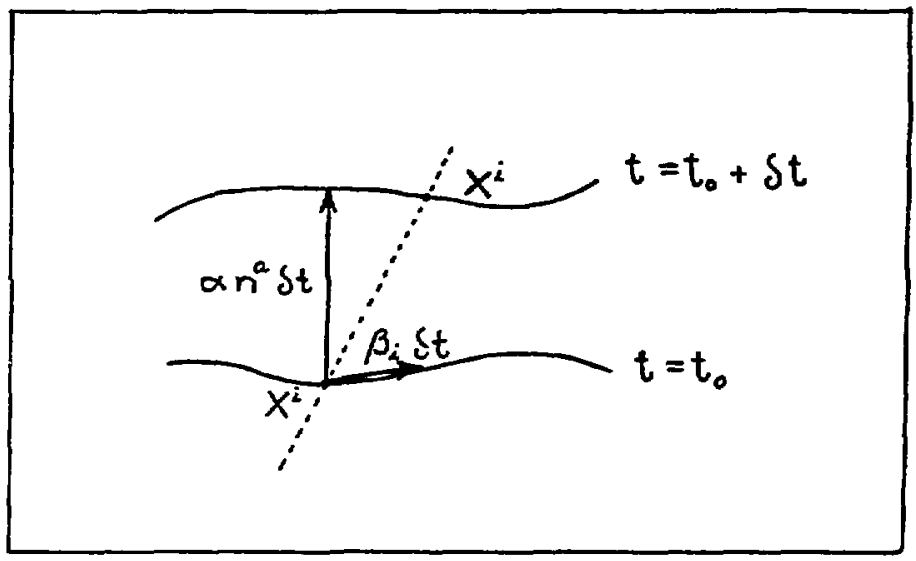

FIGURE 1 


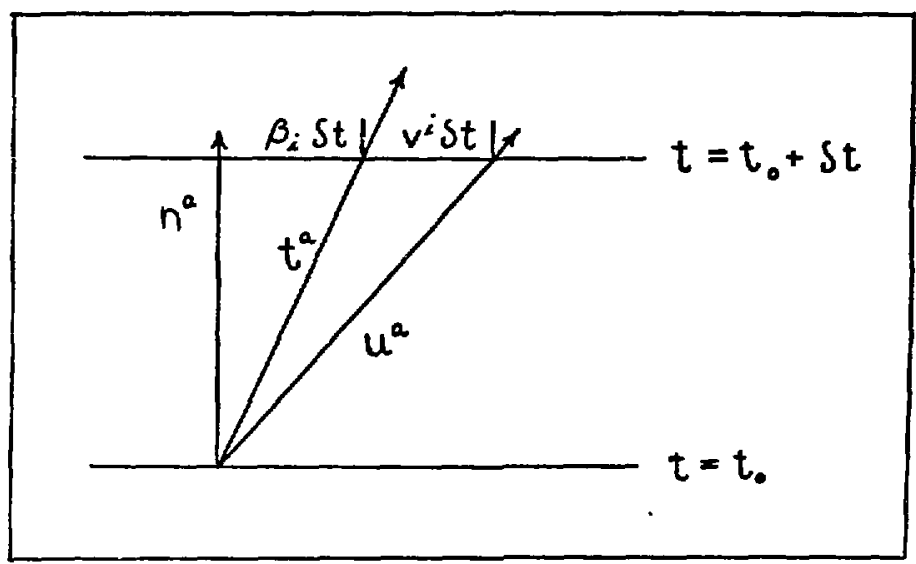

FIGURE 2 


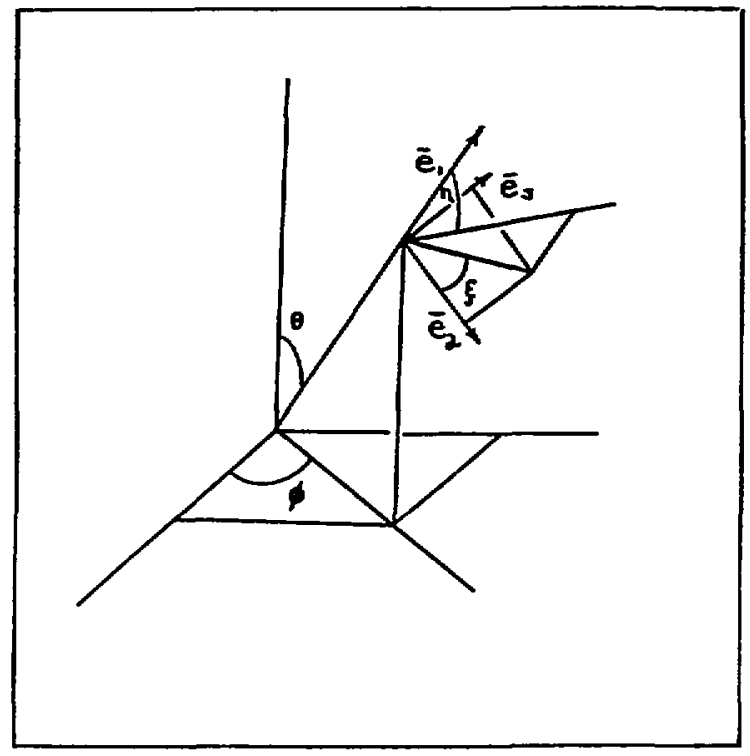

FIGURE ? 


\section{Appendix $B$}

Some miscellaneous topica will be covered in this appendix. They include the following:

1. Opacities for Neutrino Interactions

2. Thermonuclear Reaction Rateo

3. Equation of State

OPACITIES FOR NEUTRINO INTERACTIONS

The following interactions are considered in the supernova code:

Emission and Absorbtion Processes

1. neutrino emission-absorbtion by interaction with:

(a) free baryons:

i. $e^{-}+p \Longleftrightarrow n+\nu_{c}$

ii. $e^{+}+n \Longleftrightarrow p+\bar{\nu}_{e}$

(b) heavy nuclei: $e^{-}+N(Z, A) \Longleftrightarrow \nu_{c}+N^{*}(Z-1, A)$

2. pair production: $e^{-}+e^{+} \Longleftrightarrow \nu+\bar{\nu}$

3. plasma process: $\gamma_{p l} \Longleftrightarrow \nu+\bar{\nu}$

\section{Scatlering Processes}

1. scattering on free baryons or heavy nuclei:

(a) $\nu+b \Longleftrightarrow \nu+b$

(b) $\nu+N(Z, A) \Longleftrightarrow \nu+N(Z, A)$

2. scattering on electrons and positrons:
(a) $\nu+e^{-} \Longleftrightarrow \nu+e^{-}$
(b) $\nu+e^{+} \Longleftrightarrow \nu+e^{+}$ 
where the symbol $\nu$ can mean any of the three neutrinos (i.e. $\nu_{c}, \nu_{m}, \nu_{+}$) or their antiparticles. The symbol $b$ indicates either a neutron or a proton.

Note that the posoible capture interaction, $e^{+}+N(Z, A) \Longleftrightarrow \bar{\nu}_{e}+N^{*}(Z+1, A)$, in not included in the supernove code since copious $\mathrm{e}^{+}$production need temperatures greater than $.511 \mathrm{Mev}$, and for conditions where this is true, either electrons are very degenerate (i.e. in the central regions), inhibiting pooitron productior, or heavy nuclei have been dissociated by the outward moving shock wave, $\infty$ few heavy nuclei are present for the reaction. $A$ brief discussion of the cross-sections used for the neutrina interactions above will follow.

\section{EMISSTON AND ABSORBTION PROCESSES}

\section{Emiseion Absorbtion on Free Baryon:}

Using equation (2.31) from Chapter II, the croso-eection for $\nu_{c}+n \Longleftrightarrow p+e-$ can be found. This cross-section is also calculated in Tubbs and Sehramm (1975). The senumption is made that the nucleon is effectively at rest before and after the collision. The result for $\sigma\left(v_{\varepsilon} n\right)$ becomes.

$$
\sigma\left(\nu_{e} n\right)=\sigma_{o}\left(\frac{E_{\nu}+Q}{m_{c} c^{2}}\right)^{2} I\left(E_{\nu}+Q\right)\left(\frac{1+3 \alpha^{2}}{4}\right)\left(1-\left(\frac{m_{c} c^{2}}{E_{\nu}+Q}\right)^{2}\right)^{\frac{1}{2}}
$$

where $I(z)=1-1 /\left(\exp \left(\frac{z-\mu_{1}}{k_{B} T}\right)+1\right)$ is an inhibition factor that blocks the reaction if the final electron state is filled. $Q$ is the difference between the initial and final nucleon ene:gies. $E_{V}$ is the locally measured neutrino energy, $T$ is the temperature, $k_{B}$ is Boltzmann's constant, $\mu_{e}$ is the electron chemical potential, and $\alpha \approx 1.2$ ( $\approx$ stong interaction effect). $\sigma_{\mathrm{b}}=\frac{G_{p}^{2} m_{n^{2} n^{2}}^{2}}{\pi^{3} c^{2}}=1.7 \times 10^{-41} \mathrm{~cm}^{2}$, and is a typical neutrino interaction cross-section. $G_{F}$ is the weak interaction constant.

Table I contains the baryon absorbtion-emission cross-sections as used in the supernova code. In Table I , $f_{\nu}=\frac{1}{1 \pi}(h c)^{3} \int \frac{F_{2}}{D^{3}} d \mu$ is a generalized Fermi-Dirac distribution function for the peutrinos. The factor $\left(1-f_{\nu}\right)$ is a blocking factor that inhibits the reaction if a final neutrino state is already occupied. $F$ is similar to the intensity of brightness function for photon transport. The reactions listed in Table I are charged current weak interactions, and at the energies involved in a supernova, are basically uncharged from the original Fermi theory of weak interactiono.

Emissiun and Absorbtion on Heavy Nuclei 
In Chapter II, some of the considerations involved in the calculation of eapture rates on heavy nuclei are discussed. During a supernove collspse, nuclei far from the line of beta atability, found at low temperatures and densities, are formed, due to the large value of the electron chemical potential. Messured values of the needed nuclear matrix elements ujo not exist for auch elements, nor have theoretical calculations using the nuclear thell model been done, since work has heretofore concentrated on nuclei produced during atellar evolution (see Fuller (1982), Fuller et al. (1982ab)).

The approximation used in the supernova code assumes some fraction of the aeutrons and protons in the typical heavy element interact with the neutrince using the eame crosssection as given in Table I. The equations used in the supernove code to model electron capture on heavy elements will be found in Bowers and Wilson (1982a), equations (4.2) to (4.12)

\section{Pair and Plasma Processes}

For the pair processes, the following Feynmann diagrams would be used to calculate the interaction cross-sections:

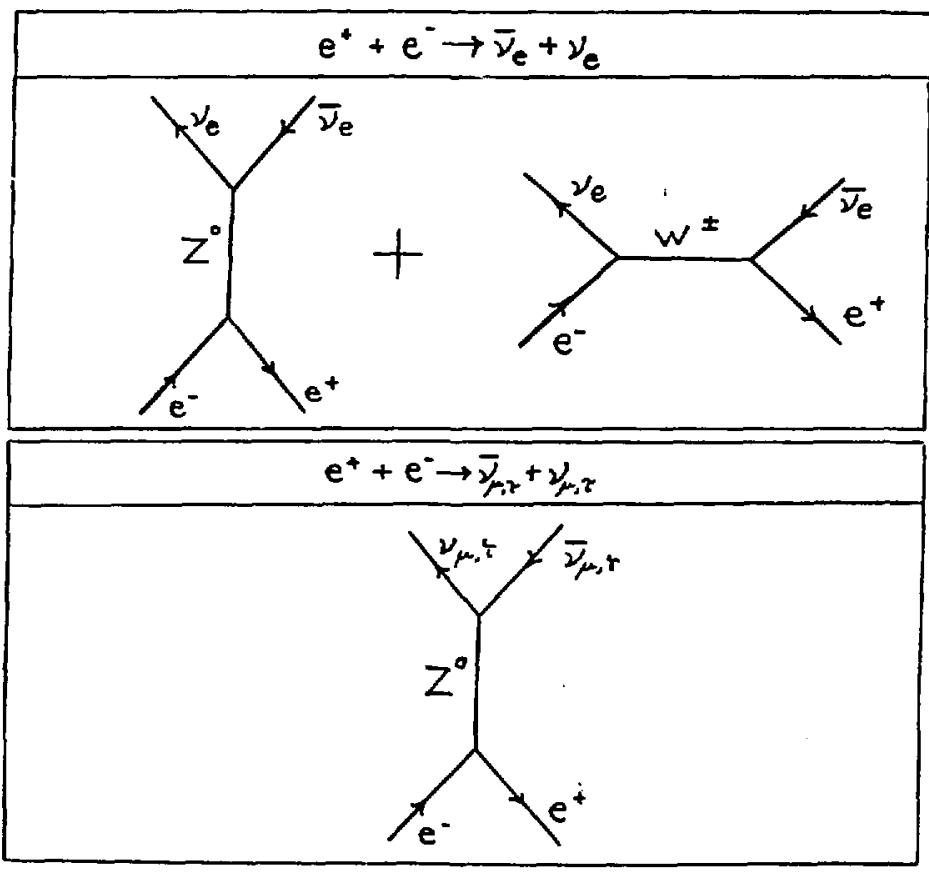


The Weinberg-Salam theory of electroweak interaction predicts the neutral current interaction, attributed to the exchange of $a Z^{0}$ boeon. This allows the production of mu and tau neutrinos and antineutrinos by $e^{-} e^{+}$annihilation.

Dicus (1972) calculated the energy production rates for the pair procesces, anuming an equilibrium distribution of electrons and positrons. A fit to the Dicue (1972) results in used, as well as a fit to the results of Chiu (1968) for the energy production in neutrinos due to the plasma process (i.e. neutrino production by a "massive" photon that acquires an effective mass by interaction with the surrounding sea of matter particles), updated with the Weinberg-Salam coupling constants, to find the neutrino apectrum produced by these processes.

Since matter is in equilibrium, the spectrum of the neutrinos produced by both processes is assumed thermal, with zero neutrino chemical potential. Only in this case will the pair production be appreciable, since electron degeneracy inhibits the pair process. This assumption is not necessarily true for the neutrino spectrum produced by the plasma process. However, the same thermal spectrum (i.e. $\left.f_{V}=1 /\left(\exp \left(\frac{\varepsilon_{v}}{k_{B} T}\right)+1\right)\right)$ is assumed for both processes. For the manner in which the pair and plasma processes, as well as the inverse reactions, is incorporated in the neutrino transport, see Bowers and Wilson(1982a), equations (8.56) to (8.65).

The present supernova code now includes the pair and plasma processes in the $\nu_{c}$ and $\bar{\nu}_{c}$ neutrino transport. The code described by Bowers and Wilson (1982a) neglected these processes for the electron neutrino and electron antineutrino, including them only for $\nu_{\mu}, \bar{\nu}_{\mu}, \nu_{r}$ and $\bar{\nu}_{r}$. The manner of incorporation of ihe new source and sink terms for $\nu_{c}$ and $\bar{\nu}_{c}$ transport is in the same spirit as that used for the mu and tau neutrinos and their antiparticles deseribed in Bowers and Wilson (1982a).

\section{SCATTERING PROCESSES}

\section{Scattering in Free Baryons or Heavy Nuclei}

The cross-sections used for scattering on free baryons and heavy nuclei are taken form Tubbs and Schramm (1975). They are listed in Table II.

\section{Scattering on Electrons and Positrons}

These scattering cross-sections are taken from a fit to numerical calculations dore by Tubbs and Schramm (1975). Thjs fit has been redone since Bowers and Wilson (1982a). Figures Ia and Ib of Chapter IV show new graphs of the cross-sections from the results 
of Tubbs and Sehramm (1975), using $\sin ^{2} \theta_{w}=.21$. The previou fit anumed $\sin ^{2} \theta_{w}=.35$. The new fit is chosen to reproduce figures la and $1 \mathrm{~b}$ for the neutrino energiem of $8 \mathrm{Mev}$ and $16 \mathrm{Mev}$ utudied by Tubbs and Schramm (1975), und is thus extrapolated to other energies.

These curves can be used to find all powible electron-ponitron seutrino-antineutrino scattering cross-sections by multiplying the fit to the curves ahown in figuree $1 \mathrm{a}$ and $\mathrm{lb}$ in .Chapter IV by an appropiate coupling constant. See Bowers and Wilenn (1982a) equation (7.3) for these coupling constants. The electron-poritron weattering crow-sections are used in calculating the mean free path of the various neutrinos types. The mean free path is used in the construction of the flux limited diffusion constants (see Chaptern III and IV).

Compton scattering of neutrinos of all types by electrons or pasitrons in also included in the supernova code using a Fokker-Planck approximation in the density region from $10^{10} \frac{\&}{c c}$ to $10^{12} \frac{2}{c e}$ where the neutrinos interact aignificantly with the matter, but not, atrong enough to thermalize completely at the matter temperature. The reaulte from a Monte Carlo calculation simulating the thermalization of neutrinos by Compton seattering with electrons, done by Tubbs (1978), is used to calibrate the Fokker-Planck equation. The Fokker-Planck equation can be derived from the Boltzmann equation only in the limit of small energy change between the initial and final neutrino energies. This is not necessarily true for neutrino Compton scattering, and so the calibration is necessary to justify the approximation. For densities less than $10^{10} \frac{\mathrm{l}}{\mathrm{ec}}$, a single scattering approximation is used to simulate the Compton scattering. For densities above $10^{12} \mathrm{e}$, the neutrinos are confined long enough for complete thermalization ts occur, mainly by interaction with free nucleors, therefore the Compton scattering with electrons and positrons is neglected. See Bowers and Wilson (1982a) for a discussion of the flux limited diffusion to neutrino transport and the method the above aross-sections are used in the oupernova code.

The present supernova code includes all electrons and positrons as scattering centers. The previous version of the supernova code only used the number of matter electrons (i.e. the number of electrons minus positrons) as possible scattering centers, and this could badly underestimate the electron-positron scattering effects.

\section{THERMONUCLEAR BURN}

Just prior to collapse, in massive stars (i.e. mass greater than $11 M_{\odot}$ ), the core is primarily iron group nuclei with an onion skin-like structure of progressively lighter elements that dominate the composition as one moves out from the center. 
For these atars, a simple nuclear reaction network is uned to follow abundances of ${ }^{12} \mathrm{C},{ }^{10} \mathrm{O},{ }^{28} \mathrm{Si}$ and $\mathrm{Fe}$. Once a sone has completely burned to $\mathrm{Fe}$, that sone in considered to be in nuclear atatistical equilibrium, with a Saha equation determining composition. It is found from numerical simulations that the energy relened by thermonuclear reactiona in not the determining factor in producing a supernora explonion, at leant not in the launching of the shock and the matter beating behind the ahock due to neutrino interactiona. However, sce Wilson et al. (1985) for a discumion of the further evolution of the shock wave a it proceeds out from $r \approx 10^{\circ} \mathrm{cm}$ to $r \approx 10^{13} \mathrm{~cm}$ (j.e. the surface of the red giant), and the impustence of esergy releaned by thermonuclear reactions.

The energy ratea per gram for ${ }^{12} \mathrm{C} \Rightarrow{ }^{10} \mathrm{O},{ }^{18} \mathrm{O} \Rightarrow{ }^{23} \mathrm{Si}$, and ${ }^{20} \mathrm{Si} \Rightarrow$ Fe are taken frt Clayton (1968). These equations and the numerical method used to implement them in the supernova code can be found in Bowers andWileon (1982a).

For stars near the masa range $8-11 M_{0}$, the core contains elements lighter than iron which undergo a collapse triggered by electron capture removing degenerate electron pressure support (see Wilson et al. (19c5)). A more complete nuciear interaction network was provided by Starford Woosley in order to study such progenitor stars, es nuclear energy generaticn may be important to the explosion.

EQUATION OF STATE

The matter particles that contribute to the pressure, energy density and rest mass density in the supernova code include: electrons, pasitrons, free neutrons and protons, helium nuclei, and one typical heavy nucleus. However, if a zone has elements present lighter than iron, nuclear statistical equilibrium does not hold, and the elements in this zone contribute in the ideal gas approximation. The massless particles that contribute to the pressure and energy density include the photons and the three neutrinos $\nu_{e}, \nu_{\mu}, \nu_{r}$ and their antjparticles. Muons, of rest mass $106 \mathrm{Mev}$, will only be present in the very central regions of the star, where the electron chemical potential can reach values of $250 \mathrm{Mev}$. A possible production resction is $e^{-} \Longrightarrow \mu^{-}+\nu_{a}+\bar{\nu}_{\mu}$. However, this is in a region where the pressure and energy density are dominated by nucleons near nuclear density, and the exact equation of state is uncertain. Muons are therefore neglected in the supernova code. The tau particle, of mass $1784 \mathrm{Mev}$, will not be produced in a supernova ( see Particle Properties Data (1984) for the masses quoted).

All the matter particles and the photons are assumed in local thermodymanic equilib 
rium at one temperature. The neutrinos are treated uaing a fux limited difruion appicuimation to neutrino transport, as previouly mentioned.

The equation of state used can be found in Bowers and Wilon (1982ab), modified to agree with the Bethe, Brown, Cooperatein and Wiloon (1893) equation of atate near nuclear density.

The electron-positron equation of state listed in Bowers and Wileon (1982a) is not ued in the present supernova code. The present code uses a tabulated electron-ponitron equation of state. Tables of pressure, energy density, and entropy were constructed by numerical integration, as functions of temperature and net electron number denvity (i.e. $n_{a^{-}}-n_{c^{+}}$). The accuracy of the numerical integration wa checked by comparasion with exact asymptotic results, plus comparasion with other tablea (i.e. Cox and Giulu (1568)). Aleo, a few points were carefully checked using error extimation formulae appropiate to the type of numerical integration used (i.e. simple midpoint integration), recognizing the fact that error formulae apply only to well behaved functions, with no infinite derivatives. One must remove any integration domain that contains such points, and estimate the error for these domains separately. It was found that the tabulated values are within $.1 \%$ of the exact values. Logarithmic interpolation is used to find the physical quanities between the grid points. In order to check on the accuracy of the interpolation, points between the tabulated values were calculated in two ways, by numerical integration and by logarithmic interpolation. It was found that the interpolation gives the physical quanities within 1 per cent of their exact value. Most of the time the interpolation is good to within .5\%. Figure 1 shows the ratio of electroil pressure to electron energy density as a fuaction of temperature, parameterized by the density. It can be seen that at the lowest density, the ratio starts at $\frac{2}{3}$ as one expects. It then drops as the temperature rises. In the temperature range where pairs are forming, the ratio drops (as low as .13 in figure 1), since the pairs take energy to produce mass that does not show up in pressure. When the temperature reaches vajues where the mass in energy units is negligible compared to the temperature in energy units, the ratio becomes $\frac{1}{3}$. As the density increases, the value of $\frac{R}{E_{s}}$ drops below $\frac{2}{3}$ nea $T=0$ Mev due to degeneracy effects. At the highest density shown the value of $\frac{P_{F}}{E_{0}}$ is $\frac{1}{\xi}$ for all the range of temperature shown. At this density, the electrons are effectively massless due io their extreme degeneracy. 


\section{Appendix B References}

Bethe, H.A., Brown, G.E., Cooperatein, J. and Wilson, J.R. 1983

Nacl. Phys. 1403, 625.

Bowera, R.L. and Wilson, J.R. 1982a Ap. J. Suppl. ,v50, 115.

-.1982b, Ap. J. ,v263, 366.

Chiu, H.Y. 1968, "Stellar Phyeics" (Waltham:Blaisdill)

Clayton, D. 1968, "Principles of Stellar Evolution and Nucleosynthesis", (McGraw-Hill:New York)

Dicus, D.A. 1972, Phys. Rev. D., v6, 941.

Fuller, G.M. 1982, Ap. J. ,v252, 741.

Fuller, G.M., Fowler, W.A., and Newman, M.J. 1982a, Ap.J. Suppl. Ser.,v48, 279.

-.1982b, Ap. J., v252, 715.

Particle Properties Data 1981, "Rev. of Mud. Phys.", v56.

Tubbs, D.L. 1978, Ap. J. Suppl., v37, 287.

Tubbs, D.L. and Schramm, D.N. 1975 Ap. J. v201, 167.

Wilson, J.R., Mayle, R., Woosley, S.E., Weaver, T.A. 1985

XII Texas Symp. Rel. Ap., in press. 
Table I. FMISSION ABSORBTION

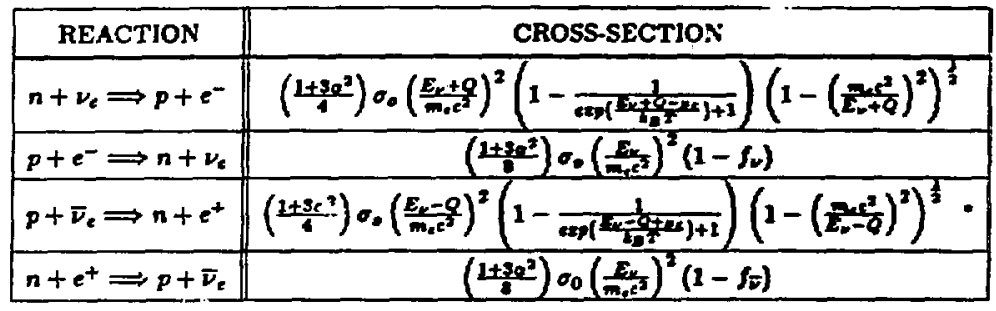

- this cross-section is zero if $E_{v} \leq Q+m_{\mathrm{a}} c^{2}$ 
Table II. SCATTERING

\begin{tabular}{|c|c|}
\hline REACTION & CROSS-SECTION \\
\hline$n+\nu \Rightarrow n+\nu$ & $\sigma_{0}\left(1-C_{A}\right)^{2}\left(\frac{E_{x}}{m_{t} \varepsilon^{2}}\right)^{2}$ \\
\hline$p+\nu \Rightarrow p+\nu$ & $\frac{1}{6} \sigma_{0}\left(\left(C_{V}-1\right)^{2}+3 \alpha^{2}\left(C_{A}-1\right)^{2}\right)\left(\frac{x_{2}}{2 \sigma^{3}}\right)^{2}$ \\
\hline$N(Z, A)+\nu \Rightarrow N(Z, A)+\nu$ & 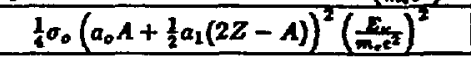 \\
\hline
\end{tabular}

In the above table, the cross-sections have been integrated over scattering angle, $a_{0}=\frac{1}{2}\left(C_{A}-C_{V}\right), a_{1}=2-C_{V}-C_{A}, C_{V}=\frac{1}{2}+2 \sin ^{2} \theta_{W}, C_{A}=\frac{1}{2}$, and $\theta_{W}$ is the Weinberg angle. Reactions in table II are mediated by the $Z^{0}$ boson and are neutral current interactions. 


\section{Appendix B Figure Caption}

Fig. 1 Ratio of electron-positron presaure to the energy density in electrons and ponitrors versus temperature for various densities. 


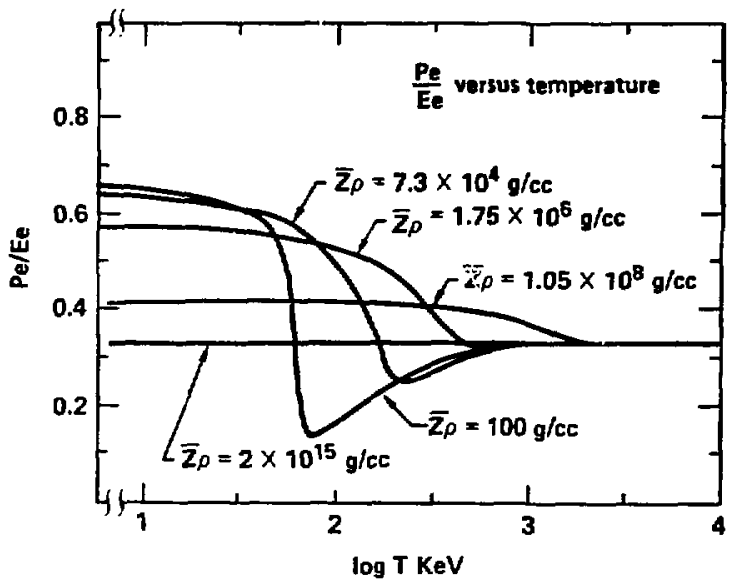

FIGURE 1 